\title{
Article
}

\author{
Doi 10.5943/sif/5/1/12
}

\section{Genera of corticioid fungi: keys, nomenclature and taxonomy}

\section{Gorjón SP}

BIOCONS - Department of Botany and Plant Physiology, University of Salamanca, 37007 Salamanca, Spain

Gorjón SP 2020 - Genera of corticioid fungi: keys, nomenclature, and taxonomy. Studies in Fungi 5(1), 125-309, Doi 10.5943/sif/5/1/12

\begin{abstract}
A review of the worldwide corticioid homobasidiomycetes genera is presented. A total of 620 genera are considered with comments on their taxonomy and nomenclature. Of them, about 420 are accepted and keyed out, described in detail with remarks on their taxonomy and systematics.
\end{abstract}

Key words - Corticiaceae - Crust fungi - Diversity - Homobasidiomycetes

\section{Introduction}

Corticioid fungi are a diverse and heterogeneous group of fungi mainly referred to basidiomycete fungi in which basidiomes are generally resupinate. Basidiome construction is often simple, and in most cases, only generative hyphae are found. In more structured basidiomes, those with a reflexed margin or with a pileate surface, more or less sclerified hyphae are usually found. Even the basidiome structure is apparently not very complex, hymenophore configuration should be highly variable finding smooth surfaces or different variations to increase the spore production area such as rugose, tuberculate, aculeate, merulioid, folded, or poroid hymenial surfaces. It is often thought that corticioid fungi produce unattractive and little variable forms and, in most cases, they go unnoticed by most mycologists as ungraceful forms that 'cover sticks and look like a paint stain'. Although the macroscopic variability compared to other fungi is, but not always, usually limited, under the microscope they surprise with a great diversity of forms of basidia, cystidia, spores and other microscopic elements (Hjortstam et al. 1987). This diversity is reflected, even adopting an inclusive vision, as it is done here, by the number of accepted genera.

Corticioid fungi are a non-natural group with species distributed in all the clades of the Basidiomycetes. They have been regarded as either a primitive group that has given rise to forms with more complicated basidiomes or as a heterogeneous assemblage of reduced forms. According to the latter hypothesis corticioid forms have evolved repeatedly through reduction from erect forms (Hibbett \& Thorn 2001, Larsson et al. 2004). In recent years, several molecular studies have contributed to elucidate the relationships between different genus of corticioid fungi (Binder et al. 2005, 2010, Larsson 2007b, Larsson \& Larsson 2003, Larsson et al. 2004).

Hjortstam (1998) provided a very complete list of corticioid fungi and subsequent authors have continued completing it with new contributions. Here, an updated review is presented, with a traditional and pragmatic approach, of all accepted and commonly used worldwide corticioid homobasidiomycetes genera and other stipitate and pileate relatives. Comments are provided on the validity and usefulness of generic concepts, always having in mind that a genus is an artificial entity that can serve to us from a didactic point of view although their limits in most cases, from morphology, biology and even from a molecular perspective, can be confusing. 


\section{Materials \& Methods}

In this review, original diagnoses and herbarium material has been used for the preparation of the keys and descriptions. Reference specimens are not cited in this revision since it is only about providing a general idea about the generic definition of corticioid fungi without conducting an indepth study of type or reference specimens, although many specimens have been studied in most cases. Keys are dichotomous, based mainly on macro- and micromorphological characters. Some genera key out by several ways in the keys; this is normal since the generic concept is artificial and, in many cases, there are no specific and diagnostic generic features. For the accepted genera, a complete description is provided with comments on nomenclature, taxonomy, and phylogeny. Here, we are considering corticioid homobasidiomycetes, mainly non-poroid species, but resupinate to effuse-reflexed polypores are also included, as well as the closest pileate and stipitate relatives. For corticioid heterobasidiomycetes the reader is referred to other specific manuals and articles.

The keys are intended to be posted on the website https://corticioids.webs.com/keys.htm, so they can be regularly updated and be rapidly accessible to the mycological community.

\section{Main key to groups of corticioid fungi and relatives}

1. Basidiome pseudostipitate to stipitate and pileate Key A

1. Basidiome sessile and resupinate to slightly pileate

2. Hymenophore poroid, with lacerate pores or with anastomosing ridges

2. Hymenophore diverse, not typically poroid

3. Spores hyaline to typically brown or violet in $\mathrm{KOH}$, ornamented, telephoric acid present. Key $\mathbf{C}$

3. Not with the above combination

4. Brown star-shaped asterohyphidia or setae present

Key D

4. Asterohyphidia or setae absent 5

5. Dichohyphae and/or binding-skeletal hyphae dextrinoid. Key $\mathbf{E}$

5. Not as above

6. Spores with a reaction in Melzer's reagent (amyloid or dextrinoid) ...........................................

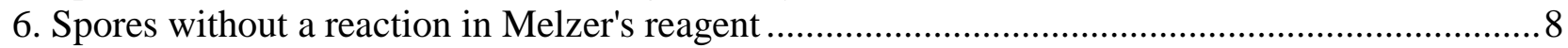

7. Spores amyloid

7. Spores dextrinoid

8. Basidia mostly with (1-3)4 sterigmata (if number of sterigmata not known follow this step)......9

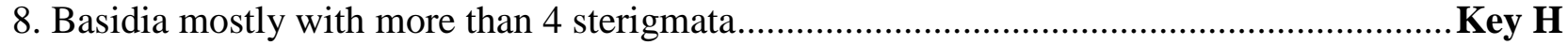

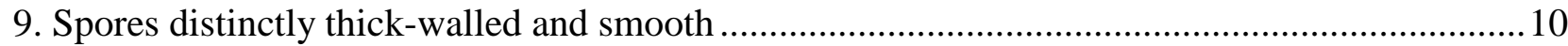

9. Spores thin-walled and/or ornamented and/or branched, lobed, triangular or tetrahedral ..........11

10. Clamps either totally absent or scattered ............................................................................. I

10. Clamps present on nearly all septa (always at the basidial base) ........................................... $\mathbf{~ J ~}$

11. Spores ornamented and/or branched, lobed or tetrahedral (easily seen in Melzer!) .............Key K

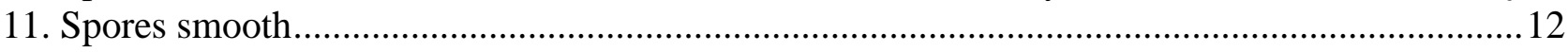

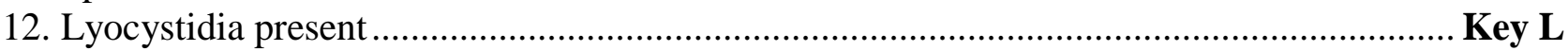

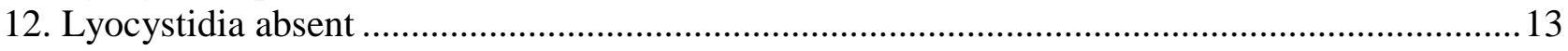

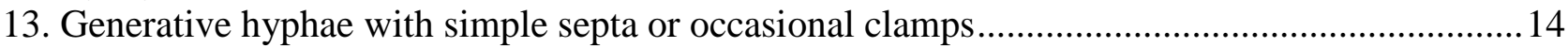

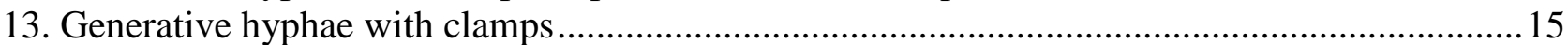

14. Cystidial organs present (excepting hyphidia or dendrohyphidia) ........................................

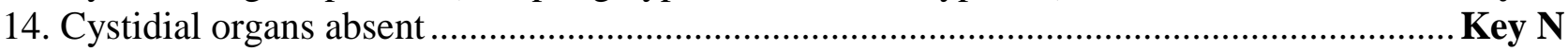

15. Hyphal system dimitic/trimitic ........................................................................................

15. Hyphal system monomitic (or pseudodimitic) ..................................................................... 16

16. Dendrohyphidia or dendrophyses present ...................................................................

16. Dendrohyphidia or dendrophyses absent (paraphysoid hyphae may be present).......................17

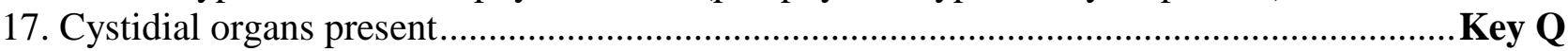

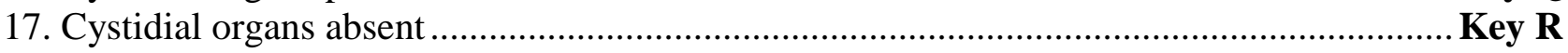




\section{Key A: Basidiome pseudostipitate to stipitate and pileate}

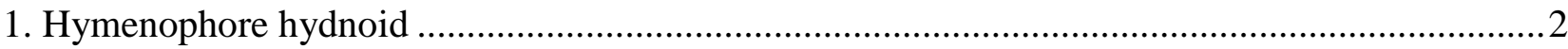

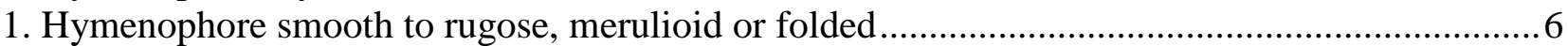

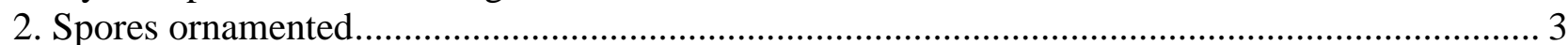

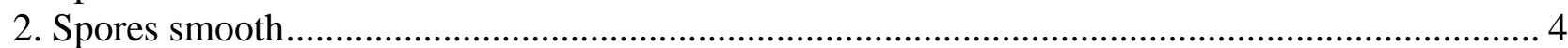

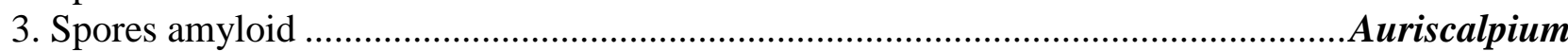

3. Spores non-amyloid ........................................................................ (see Trechispora) Hydnodon

4. Basidiome fragile, basidia 6-sterigmate, hyphae with abundant oily contents................Sistotrema

4. Basidiome tough, basidia 4-sterigmate, hyphae not as above ................................................. 5

5. Hyphal system monomitic, gloeocystidia absent......................................................eptodonoides

5. Hyphal system dimitic, gloeocystidia usually present.......................................... Mycorrhaphium

6. Basidiome infundibuliform, with a hollow stipe .................................................... Parastereopsis

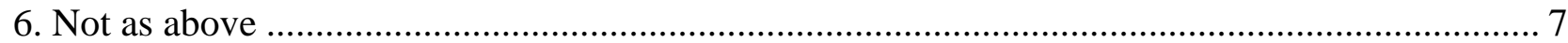

7. Hyphal system monomitic (check for vegetative hyphae in the stipe!),.................................... 8

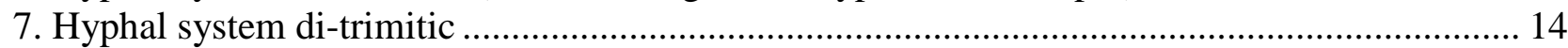

8. Cystidia present (patent and projecting above the basidial layer) ........................................... 9

8. Cystidia absent (may be present cystidiols or gloeocystidia but little differentiate or embedded) .

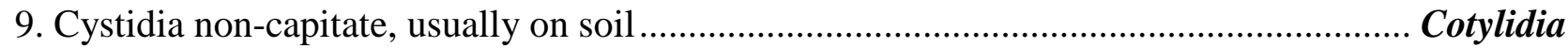

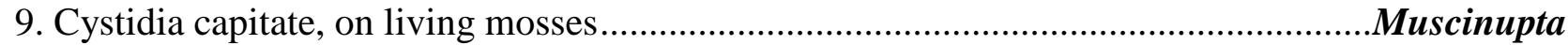

10. Basidiome white, obconical to discoid with a central stipe ................................................ Caripia

10. Not as above, basidiome usually flabelliform …..................................................................11

11. Coralloid hyphae present in the pileal surface and stipe ....................................... Coralloderma

11. Not as above, coralloid, or dendroid hyphae absent ............................................................... 12

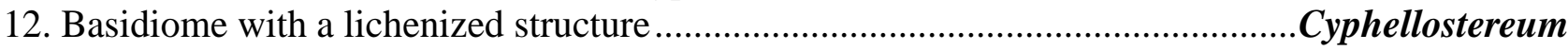

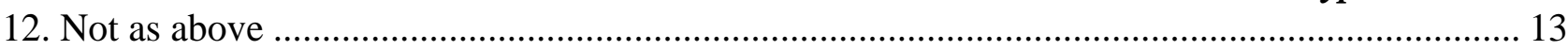

13. Hymenophore merulioid, spores thick-walled...........................................................odoserpula

13. Hymenophore smooth to rugose, spores thin-walled ....................................................... Stereopsis

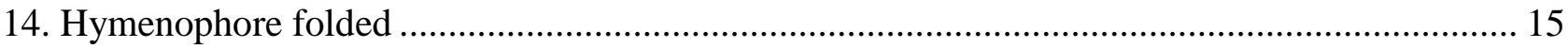

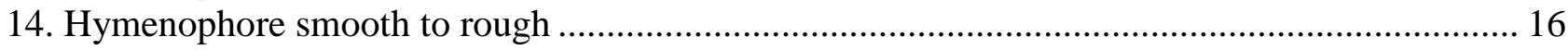

15. Hyphae inflated in context, cystidia absent ........................................................... Inflatostereum

15. Hyphae non-inflated, cystidia encrusted and gloeocystidia present .......................... Cymatoderma

16. Hyphal system trimitic, gloeocystidia absent .......................................................... Aquascypha

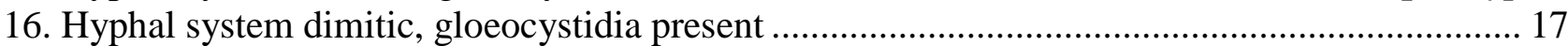

17. Generative hyphae simple-septate, dextrinoid binding hyphae, spores amyloid... Dichopleuropus

17. Generative hyphae clamped, skeletal hyphae non-dextrinoid, spores non-amyloid ....Podoscypha

Key B: Basidiome usually sessile and resupinate to pileate, hymenophore poroid with lacerate pores or with anastomosing ridges

1. Basidiome brown, becoming black in $\mathrm{KOH}$, generative hyphae with simple-septa, setae present or absent .(Hymenochaetaceae) 2

1. Not with this combination of characters 3

2. Basidiome perennial, hard, hyphal system dimitic....

Phellinus s.l.

2. Basidiome annual, soft, and fragile when dry, hyphal system monomitic Inonotus s.l.

3. Pores developing from an apical pore in isolated papillae. (=Stromatoscypha) Porotheleum

3. Pores development not as above

4. Hymenium restricted to the base of the pores, tube walls sterile ................................................ 5

4. Hymenium covering base and tube walls (may be absent in the dissepiments) .......................... 9

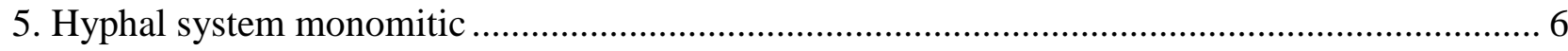

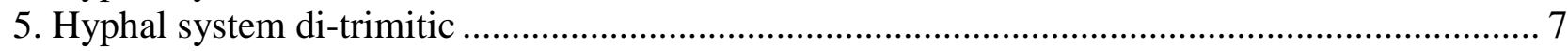


6. Hymenophore with irregular pores, substate reddened

Porogramme

6. Hymenophore with long anastomosing ridges, substrate not reddened ................Hymenogramme

7. Basidiospores thick-walled, with dextrinoid arboriform hyphae

Grammothelopsis

7. Basidiospores thin-walled, arboriform hyphae if present non-dextrinoid

8. Skeletal hyphae present, darkening with age and dextrinoid

Grammothele

8. Arboriform hyphae present, not darkening with age, non-dextrinoid Theleporus

9. Basidiospores with a reaction in Melzer's reagent....

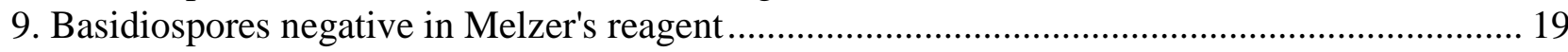

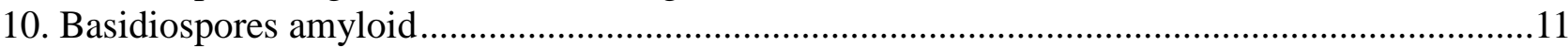

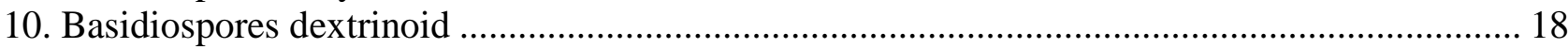

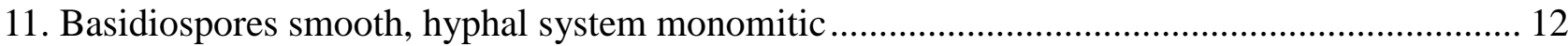

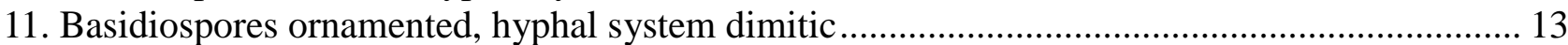

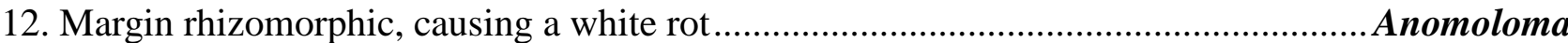

12. Margin not rhizomorphic, causing a brown rot ..........................................................nomoporia

13. Generative hyphae with simple septa or alternating multiple clamps connections ....Amylosporus

13. Generative hyphae with clamps.

(Wrightoporia s.l.)14

14. Pores labyrinthine to daedaleoid

Amylonotus

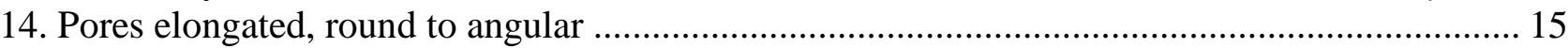

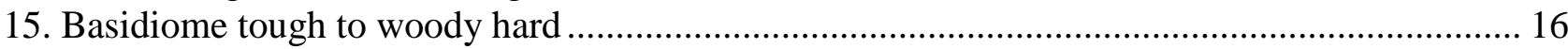

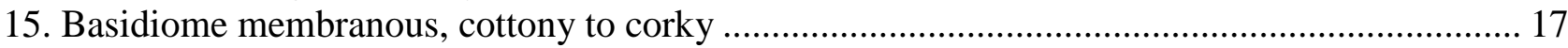

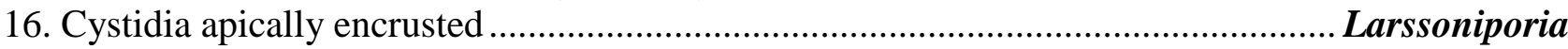

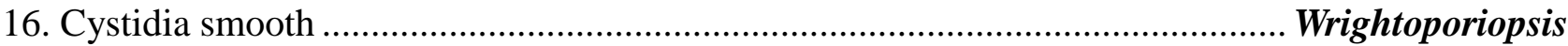

17. Basidiome membranous to cottony, margin usually with rhizomorphs..................... Wrightoporia

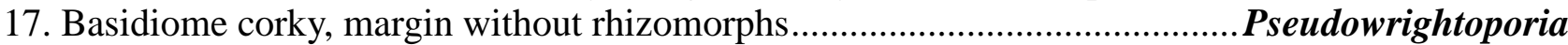

18. Hyphal system monomitic ..............................................................(=Parmastomyces) Sarcoporia

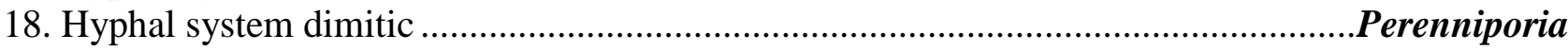

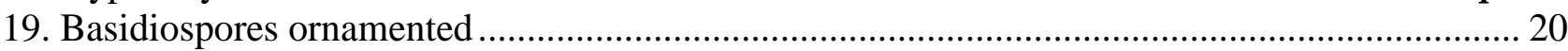

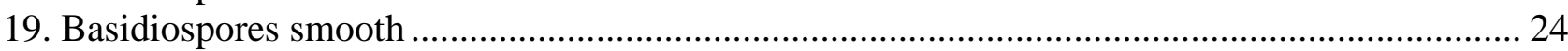

20. Spore ornamentation rough based on longitudinal striae or rounded ridges ....

(=Pachykytospora) Haploporus

20. Spore ornamentation asperulate, echinulate or aculeate ….................................................... 21

21. Hyphal system monomitic in most species, hyphae with clamps......................................... 22

21. Hyphal system dimitic, generative hyphae with simple septa................................. Heterobasidion

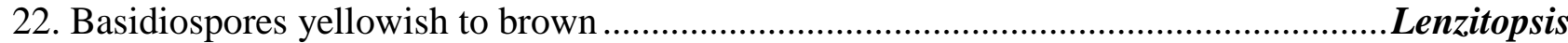

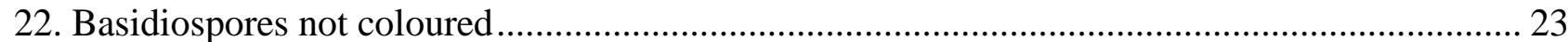

23. Hyphae usually ampullate at septa, basidia with no cyanophilous granulation ............Trechispora

23. Hyphae not ampullate at septa, basidia with cyanophilous granulation ...........................indtneria

24. Hyphae with simple septa (some scattered clamps may occur in some hyphae) ...................... 25

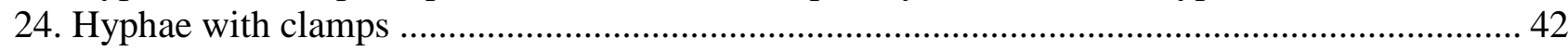

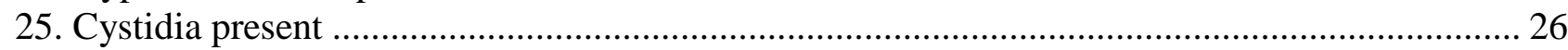

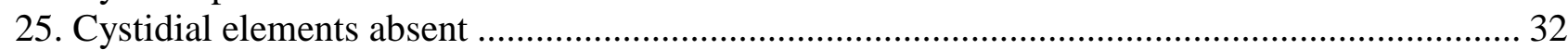

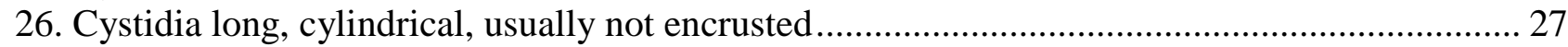

26. Cystidia slender, usually encrusted at the apex .................................................................... 28

27. Basidiome white to cream, not changing in $\mathrm{KOH}$, causing a white rot................... Leucophellinus

27. Basidiome orange to red, red in $\mathrm{KOH}$, causing a brown rot.....................................Pycnoporellus

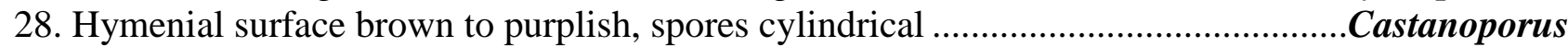

28. Hymenial surface whitish to orange, spores widely ellipsoid to subglobose ............................. 29

29. Hymenial surface vivid orange to dark colored, mucronate cystidiols present............ Rigidoporus

29. Hymenial surface paler, mucronate cystidiols absent............................................................... 30

30. Hymenial surface with yellow brown colors, effuse-reflexed to pileate ........................ Oxychaete

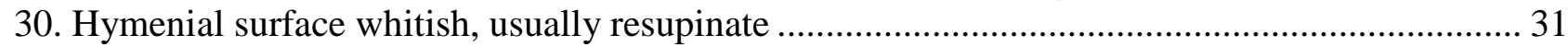

31. Hyphal system monomitic, hyphae slightly amyloid ....................................................... Emmia 
31. Hyphal system dimitic or pseudodimitic, hyphae not amyloid ..... Oxyporus

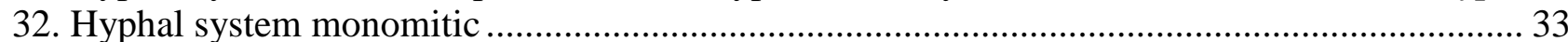

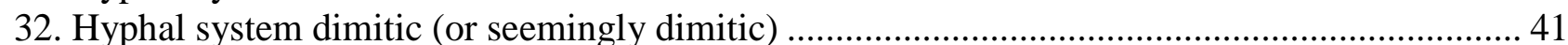

33. Basidiospores thick-walled

Byssoporia

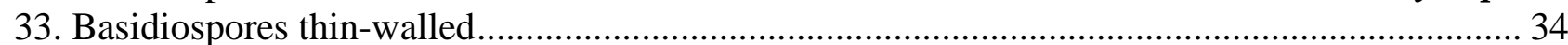

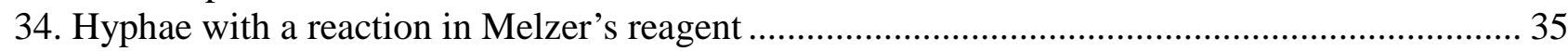

34. Hyphae without a reaction in Melzer's reagent .................................................................. 36

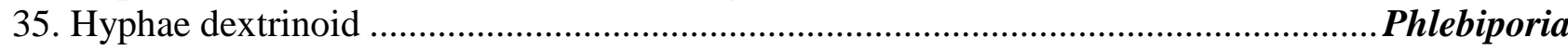

35. Hyphae slightly amyloid (some hyphae with scattered clamps) ............................. Elaphroporia

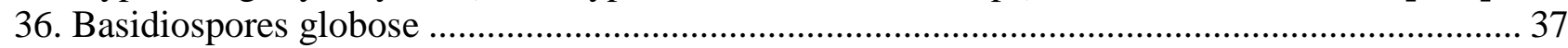

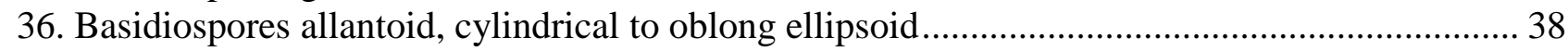

37. Basidiome resupinate to pileate, coriaceous, basal hyphae thick-walled .................... Rigidoporus

37. Basidiome resupinate, ceraceous, basal hyphae thin-walled ..................................Physisporinus

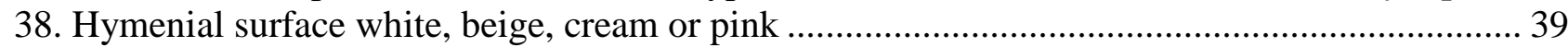

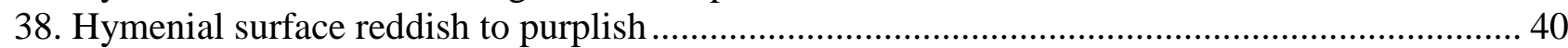

39. Pore surface white to buff, anamorph present, basidiospores allantoid......................................opa

39. Pore surface more variable in color, anamorph absent, basidiospores variable ................Ceriporia

40. Hymenophore merulioid with shallow pits, basidiome thin, not pileate .........................eruliopsis

40. Hymenophore typically poroid, basidiome fleshy, often pileate ...................................eptoporus

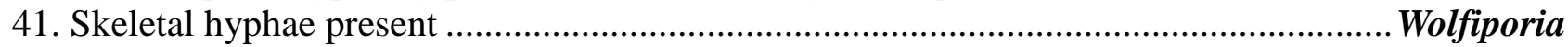

41. Binding hyphae or sclerified tortuous generative hyphae present ..............................acrohyporia

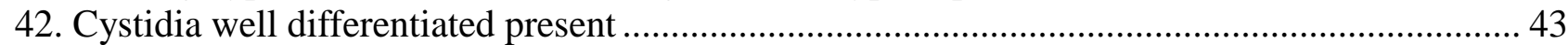

42. Cystidia absent (cystidiols, dendrohyphae or encrusted hyphae may be present)....................... 55

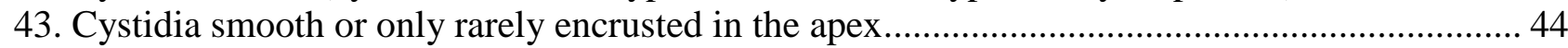

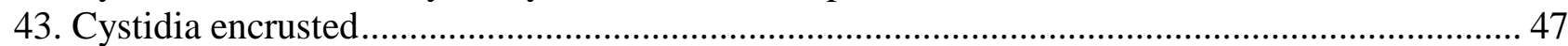

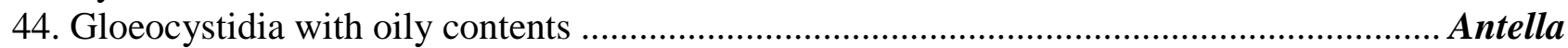

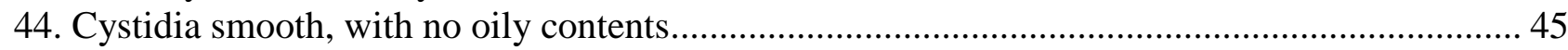

45. Hymenophore poroid to lamellate, with brown colors, causing a brown rot ..... Gloeophyllum s.l.

45. Hymenophore poroid, light colored, causing a white rot ........................................................ 46

46. Basidiome white, cystidia cylindric, spores narrowly allantoid ...... (=Chaetoporellus) Kneiffiella

46. Basidiome yellow to straw-coloured, cystidia hyphae-like, spores ellipsoid ................. Luteoporia

47. With two types of cystidia: 1) tramal thick-walled encrusted cystidia, 2) gloeocystidia ... Butyrea

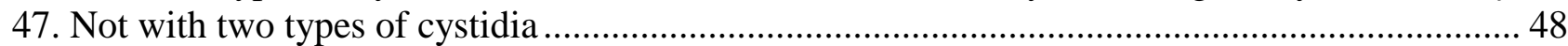

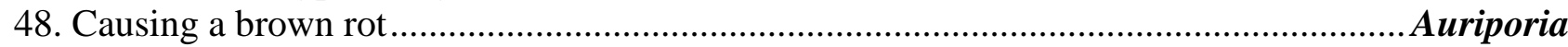

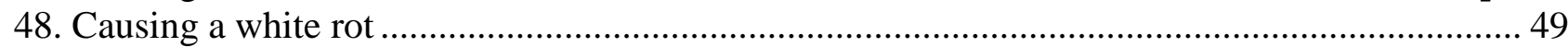

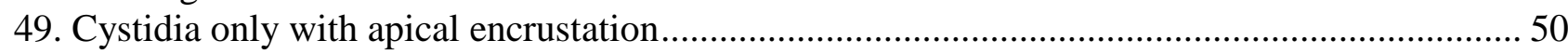

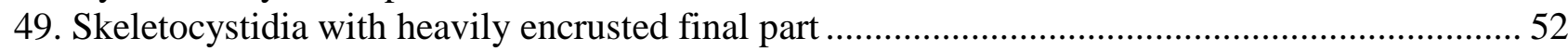

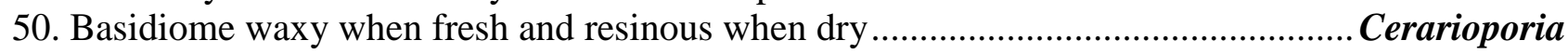

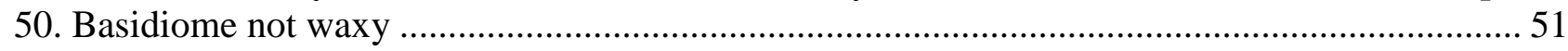

51. Hymenial surface with violet shades, hyphal system dimitic.................................... Trichaptum

51. Hymenial surface with no violet shades, hyphal system monomitic..................................Xylodon

52. Hymenial surface vivid chrome yellow ….............................................................. Flaviporus

52. Hymenial surface cream-coloured to pinkish, buff, cinnamon............................................... 53

53. Hymenial surface reddish when bruised, skeletal hyphae metachromatic ...................Geesterania

53. Hymenial surface usually not changing, skeletals not metachromatic ..................................... 54

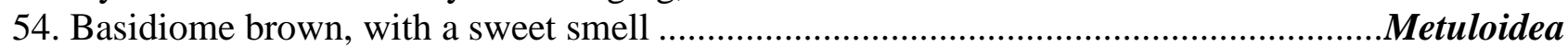

54. Basidiome usually not brown, with no sweet smell ........................(=Junghuhnia) Steccherinum

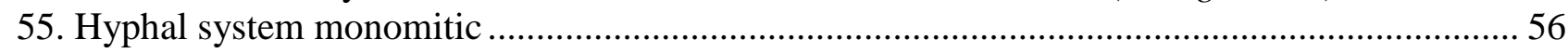

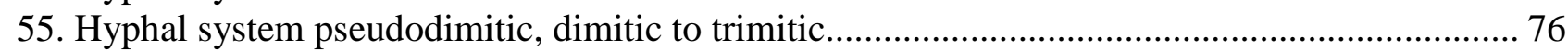

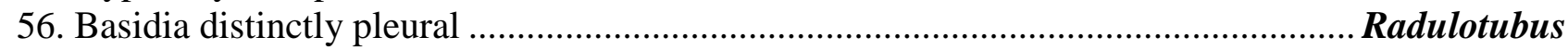

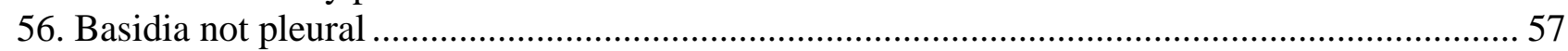

57. Basidia urniform, with 4-8 sterigma, hyphae with oily contents, usually ampullate ......Sistotrema 
57. Basidia clavate or cylindrical, with 4-sterigma, hyphae not ampullate and with no oily contents .

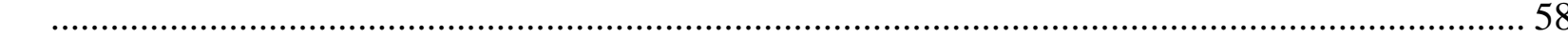

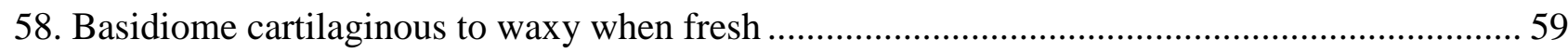

58. Basidiome not cartilaginous or waxy when fresh (ceraceous follow this step)........................ 64

59. Pore surface whitish, stained dark brown to black when bruised. Atraporiella

59. Pore surface not blackening when touched

60. Hymenophore pink to reddish and more or less gelatinous, dissepiments fertile

.(see also Vitreoporus) Gloeoporus

60. Hymenophore usually differently coloured and non-gelatinous, dissepiments usually sterile.... 61

61. Basidiome changing red in $\mathrm{KOH}$.

(see also Erastia) Aurantiporus

61. Basidiome not changing in $\mathrm{KOH}$....

62. Basidiospores globose, broad bottle-shaped cystidiols

Obba

62. Basidiospores cylindrical to allantoid, no broad bottle-shaped cystidiols.........

63. Basidiospores allantoid, hyphae swelling and gelatinized in $\mathrm{KOH}$.

63. Basidiospores cylindrical, hyphae not swelling and gelatinized in $\mathrm{KOH}$

Gelatoporia

64. Basidiome with rhizomorphs, hyphae encrusted. Sebipora

64. Basidiome with no rhizomorphs, hyphae encrusted or not. Porpomyces

65. Basidiome usually changing red or purple in $\mathrm{KOH}$. 65

65. Basidiome not changing in $\mathrm{KOH}$. (see also Erastia) Hapalopilus

66. Basidiome reddening after bruising, with skeletoid hyphae. 66

66. Basidiome not reddening after bruising, skeletoid hyphae absent Pouzaroporia

67. Basidiospores globose 67

67. Basidiospores allantoid, cylindrical to oblong ellipsoid.

Physisporinus

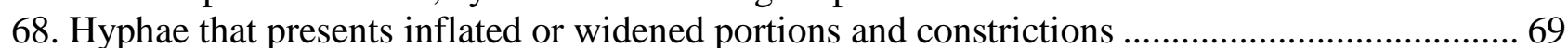

68. Hyphae not as above

69. Basidiospores allantoid, hyphae thick-walled, irregularly inflated and constricted. Aegis

69. Basidiospores subglobose, hyphae thin-walled, with ampullaceous septa Caudicicola

70. Causing a white rot..... 71

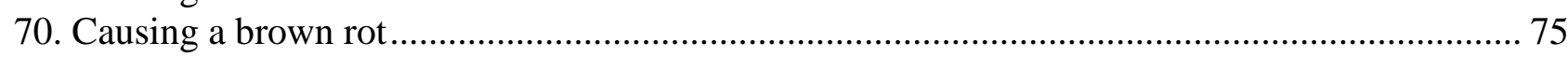

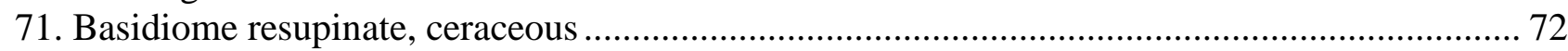

71. Basidiome resupinate to effused-reflexed to pileate, soft to corky ............................................ 73

72. Basidiospores globose to subglobose

Niemelaea

72. Basidiospores ellipsoid to cylindrical

Ceriporiopsis

.(see also Frantisekia, Raduliporus, Resiniporus)

73. Tubes white to yellowish

Tyromyces

73. Tubes greyish-violaceous to brown 74

74. Basidiome soft, very brittle when dry, basidiospores allantoid .... Fragiliporia

74. Basidiome corky, basidiospores cylindrical Bjerkandera

75. Basidiome mostly white to yellowish Postia

75. Basidiome pinkish to red Rhodonia

76. Contextual skeletal hyphae cyanophilous. 77

76. Contextual skeletal hyphae not cyanophilous. 79

77. Skeletal hyphae dextrinoid

77. Skeletal hyphae non dextrinoid

Megasporoporia

78. Skeleto-binding hyphae in context arboriform, resinous orange substance in context

78. No arboriform skeletal hyphae, resinous substance in context absent ....

Gloeoporellus

79. Skeletal hyphae with a variable reaction in Melzer's reagent.

Cyanotrama

79. Skeletal hyphae not reacting in Melzer's reagent

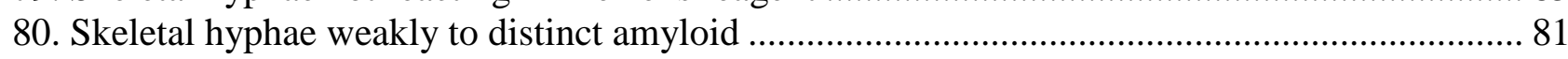

80. Skeletal hyphae usually dextrinoid 83

81. Causing a brown rot. 
82. Basidiospores allantoid, thin-walled. (=Canopora?) Cinereomyces

82. Basidiospores ellipsoid, thick-walled (see also Amylosporia) Yuchengia

83. Substrate reddened in zones Tinctoporellus

83. Substrate not reddened.

84. Basidiospores negative in Melzer's reagent Megasporia

84. Basidiospores with a variable dextrinoid reaction. Perenniporia

85. Hyphal system pseudodimitic, skeletoid hyphae or skeletal hyphae present only in context ..... 86

85. Hyphal system dimitic to trimitic 87

86. Thick-walled hyphae with sparse clamps, metachromatic Rickiopora

86. Skeletal hyphae present only in context, not metachromatic Frantisekia

87. Basidiome woody, dark brown, context brown ..... . .88

87. Not as above, context light or pale coloured 94

88. Hymenophore poroid, daedaleoid to lamellate, causing a brown rot Gloeophyllum s.l.

88. Hymenophore poroid with round to angular pores, causing a white rot 89

89. Basidiome resupinate. 90

89. Basidiome usually effuse-reflexed to pileate. 91

90. Dendrohyphidia present in the dissepiments, basidiospores cylindrical Neodatronia

90. Dendrohyphidia absent, basidiospores ellipsoid Donkioporia

91. Context rusty brown, no black line between context and tomentum, binding hyphae present

Funalia

91. Context dark brown, with a black line between context and tomentum, binding hyphae rare.... 92

92. On conifers, basidiospores allantoid, 3-4 $\mu \mathrm{m}$ long

Piloporia

92. On hardwoods, basidiospores cylindrical, 8-12 $\mu \mathrm{m}$ long . 93

93. Dendrohyphidia present in the dissepiments Datronia

93. Dendrohyphidia absent in the dissepiments Datroniella

94. Hyphal system trimitic, with skeletal and binding hyphae. Earliella

94. Hyphal system dimitic, with skeletal or binding arboriform hyphae 95

95. Binding arboriform vegetative hyphae, dichotomously branched. (see also Szczepkamyces) Dichomitus

95. Skeletal hyphae more or less straight

96. Causing a white rot, spores usually small.

96. Causing a brown rot, spores usually large Antrodia s.l 101

(see also Antrodiopsis, Brunneoporus, Dentiporia, Flavidoporia,

Lentoporia, Neoantrodia, Rhizoporia, Subantrodia)

97. Skeletal hyphae more or less finely encrusted.

97. Skeletal hyphae not encrusted

98. Pores round to lacerate to hydnaceous, basidiospores ellipsoid ..(=Schizopora $)$ Xylodon

98. Pores usually round, small, basidiospores allantoid to cylindrical Skeletocutis

99. Basidiospores ellipsoid to cylindrical, usually up to $5 \mu \mathrm{m}$ long Antrodiella

99. Basidiospores usually allantoid to cylindrical or ellipsoid, usually longer than $5 \mu \mathrm{m}$. 100

100. Basidiospores allantoid to cylindrical, basidiome tough Diplomitoporus

100. Basidiospores ellipsoid, basidiome soft.... Cinereomycetella

101. Basidiome soft and fleshy, hymenophore gelatinous Cartilosoma

101. Basidiome tough to hard, hymenophore not gelatinous 102

102. Subiculum cottony with brown generative hyphae next to the substrate Anthoporia

102. Not as above 103

103. Skeletal hyphae swelling and dissolving in $\mathrm{KOH}$, weak to distinctly amyloid. Amyloporia

103. Skeletal hyphae not swelling and dissolving in $\mathrm{KOH}$, not amyloid 104

104. Resinous matter abundant present as droplets or globose bodies Resinoporia

104. Not as above 105

105. Rhizomorphs absent, basidiospores allantoid, cylindrical to narrowly ellipsoid Antrodia 
105. Rhizomorphs usually present, basidiospores usually ellipsoid 106

106. Basidiospores thin-walled, skeletal hyphae thinner than generative hyphae

Leifiporia

106. Basidiospores firm to thick-walled, skeletal hyphae wider than generative hyphae 107

107. Basidiomes resupinate with rhizomorphic margin

107. Basidiospores pileate with entire margin....

Fibroporia Pseudofibroporia

Key C: Basidiome usually resupinate, hymenophore non-typically poroid, spores hyaline to typically brown or violet in $\mathrm{KOH}$, ornamented, telephoric acid present (tomentelloid fungi)

1. Basidiome bluish when fresh, greenish when dry, spores violaceous in $\mathrm{KOH}$ Amaurodon

1. Not as above 2

2. Hyphae simple-septate, spores echinulate and hyaline in $\mathrm{KOH}$ Tomentellopsis

2. Not with this combination of characters 3

3. Basidiome effuse to effuse-reflexed, hymenophore irpicoid to lamellate Lenzitopsis

3. Not as above . .4

4. Basidia sphaeropedunculate, hyphae mostly simple-septate or clamped with hyaline spores

Pseudotomentella

4. Basidia usually clavate, hyphae mostly clamped and spores mostly brownish in $\mathrm{KOH}$. (see also Odontia for non-mycorrhizal species) Tomentella

Key D: Basidiome usually resupinate, hymenophore non-typically poroid, smooth or hydnoid, with brown asterohyphidia or setae

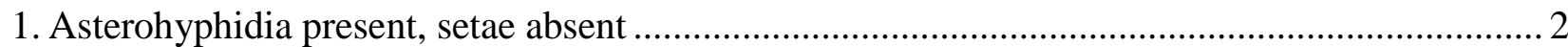

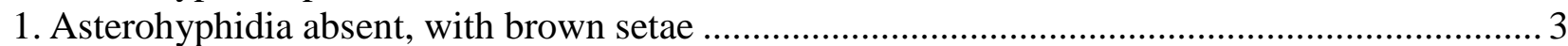

2. Hymenophore smooth, asterohyphidia dextrinoid, spores amyloid .... Asterostroma

2. Hymenophore hydnoid, asterohyphidia not dextrinoid, spores inamyloid. Asterodon

3. Basidiospores narrowly cylindrical to allantoid Hymenochaetopsis

3. Basidiospores cylindrical, ellipsoid to subglobose. (includ. Hydnochaete) Hymenochaete

Key E: Basidiome usually resupinate, hymenophore non-typically poroid, without asterohyphidia; setae absent, dichohyphae and/or dextrinoid binding-skeletal hyphae present

1. Dichohyphae present 2

1. Dichohyphae absent (dendrohyphidia may be present)........ (see also Baltazaria) Scytinostroma

2. Spores smooth, amyloid or not Vararia

2. Spores verrucose, strongly amyloid. Dichostereum

Key F: Basidiome usually resupinate, hymenophore non-typically poroid, without asterohyphidia; setae absent, dichohyphae and/or dextrinoid binding-skeletal hyphae absent; spores amyloid

1. Hymenophore clearly odontioid to hydnoid 2

1. Hymenophore smooth to verrucose or slightly merulioid 11

2. Basidiome stipitate, growing on cones of Picea or Pinus

2. Basidiome different Auriscalpium

3. Aculei arising single, usually inconspicuous 3

3. Aculei disposed on a structured conspicuous basidiome

4. Cystidia absent.

4. Cystidia present

5. Spores smooth. ... 4

5. Spores ornamented. Mucronella Dentipratulum .. Irpicodon 6 


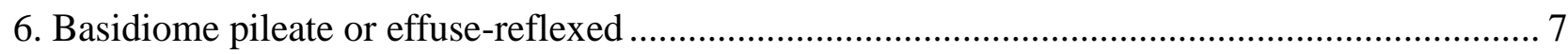

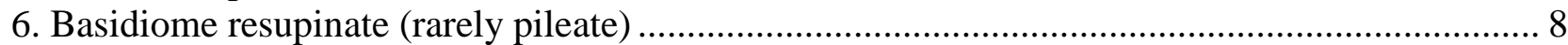

7. Basidiome pileate, fleshy, globose to ramified or dimidiate, white to cream....................Hericium

7. Basidiome effuse-reflexed, not as above, brownish to greyish Gloiodon

8. Hyphal system dimitic or pseudodimitic, metuloids present Gloeodontia

8. Hyphal system monomitic, metuloids absent

9. Basidiospores distinctly thick-walled, mostly $>5.5 \mu \mathrm{m}$ long (Dentipellis s.l.) 9

9. Basidiospores slightly thick-walled, mostly $<5.5 \mu \mathrm{m}$ long Dentipellopsis

10. Generative hyphae CB10

10. Generative hyphae $\mathrm{CB}+$

Dentipellicula

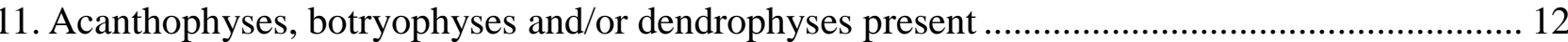

11. Acanthophyses, botryophyses and/or dendrophyses absent ................................................... 21

12. Hyphal system dimitic (compare also Xylobolus and Perplexostereum) ............................Stereum

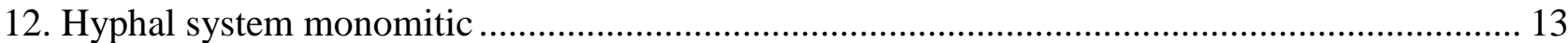

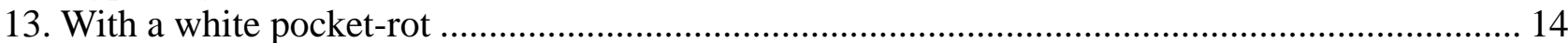

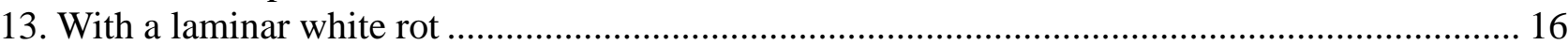

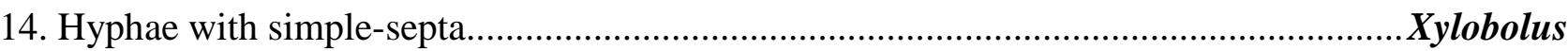

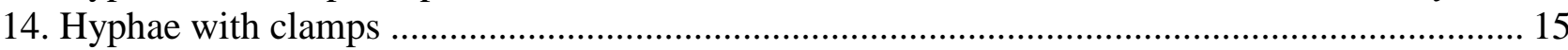

15. Basidiome pulvinate, with acanthophyses, spores smooth...................................Acanthofungus

15. Basidiome effused, no acanthophyses, spores finely warted.............................. Haloaleurodiscus

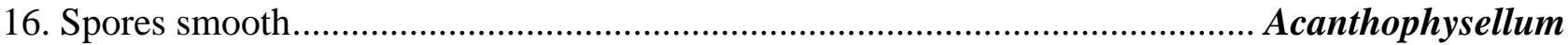

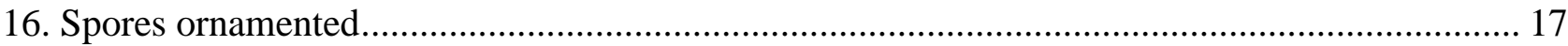

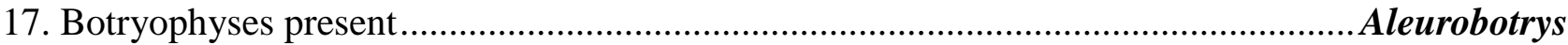

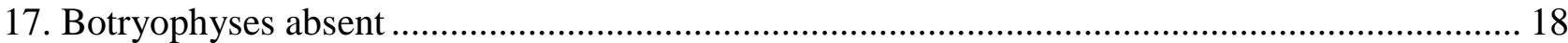

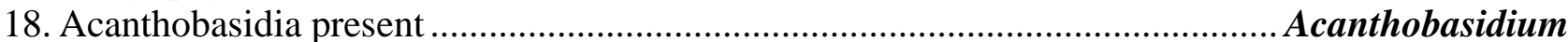

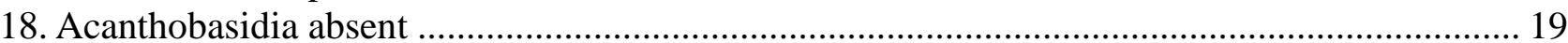

19. Basidiome fleshy and gelatinous, cupulate, yellowish to orange coloured .................... Gloeosoma

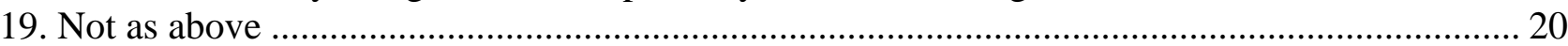

20. Spore print white, with acanthophyses, hyphae simple-septate ...............................Aleuromyces

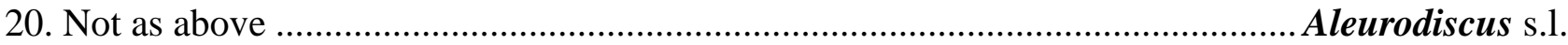

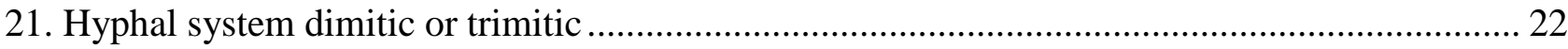

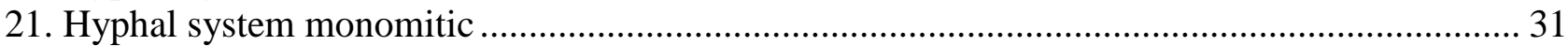

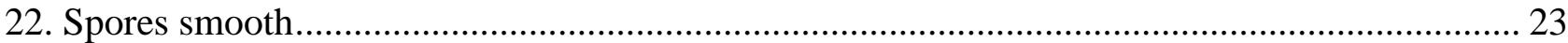

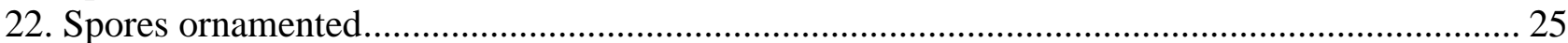

23. With brown, encrusted (metuloid) cystidia...

Amylostereum

23. Without brown, encrusted (metuloid) cystidia............................................................................ 24

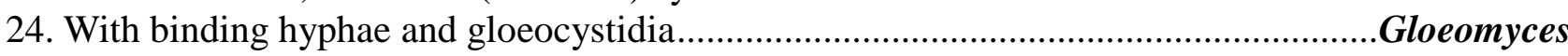

24. Without binding hyphae and gloeocystidia.................................................................... Stereum

25. Spores 15-20 $\mu \mathrm{m}$ long ................................................................................... Aleurocystidiellum

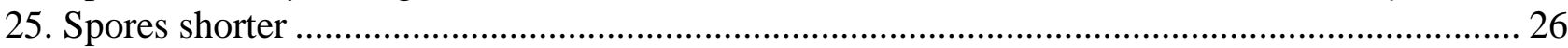

26. Gloeocystidia (SA+) present.............................................................................. Scytinostromella

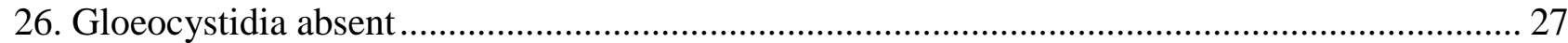

27. Hyphal system trimitic, pseudocystidia absent (encrusted cystidia present)...................... Laurilia

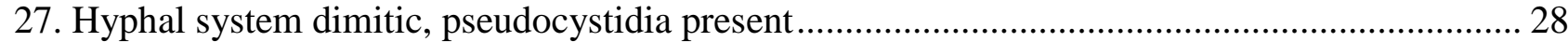

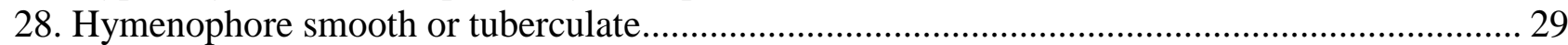

28. Hymenophore odontioid to hydnoid or dedaleoid .................................................................... 30

29. Context reddish-brown, pseudocystidia smooth..................................................Perplexostereum

29. Context light orange, pseudocystidia encrusted ............................................................. Lauriliella

30. Basidiome effuse-reflexed to pileate, context brick-red to brownish orange, on conifers

Echinodontium

30. Basidiome effused to effuse-reflexed, context cinnamon to olive grey, on Quercus ..... 


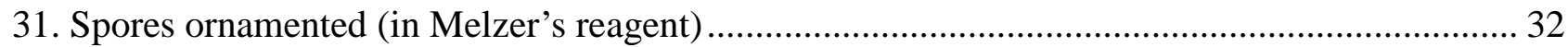

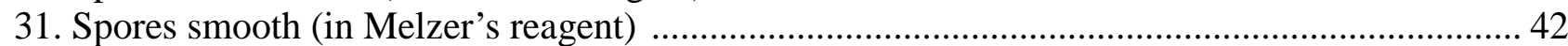

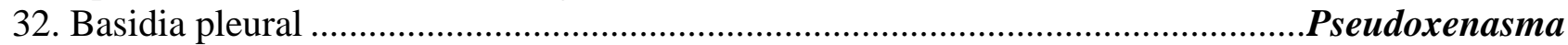

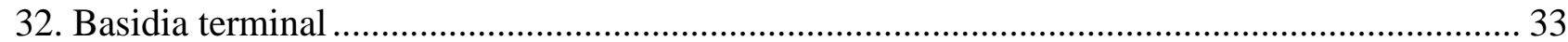

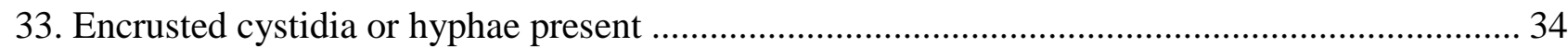

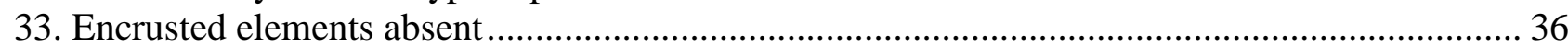

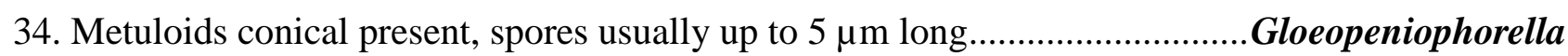

34. Encrusted elements not typical as conical metuloids, spores longer than $5 \mu$ m........................ 35

35. Cystidia encrusted, spores ornamented with bifurcate aculei...............................Aleurodiscus s.l.

35. Encrusted elements as skeletocystidia, spores verrucose ................................ Aleurocystidiellum

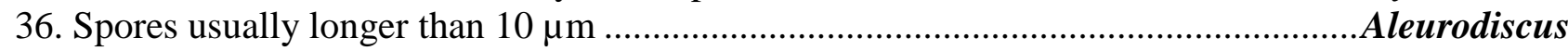

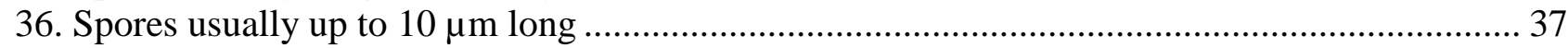

37. Basidiome pellicular, gloeocystidia absent.............................................................. Amyloathelia

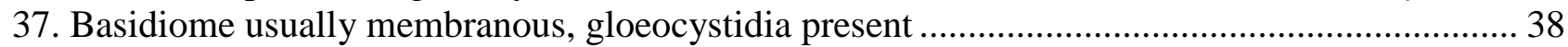

38. Basidiome stereoid, pileate or resupinate, context dark brown .................................. Laxitextum

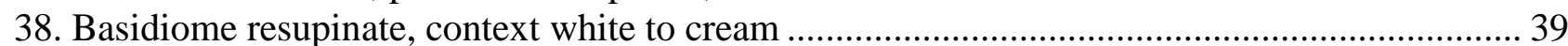

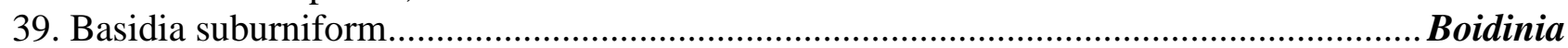

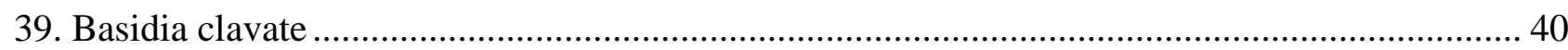

40. Context pseudoparenchymatic, hyphae cyanophilous, basidia with internal repetition.....

Conferticium

40. Not as above

41. Basidiome vivid orange, hyphae simple septate, tropical genus ......................... Gloeocystidiopsis

41. Basidiome generally paler, hyphae with or without clamps, widespread............Gloeocystidiellum

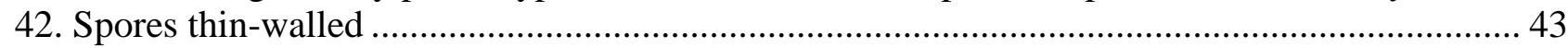

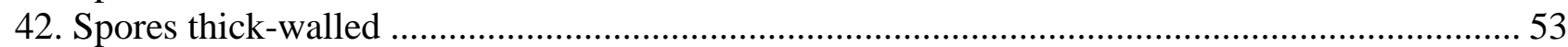

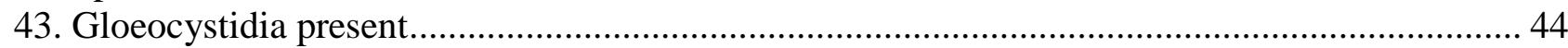

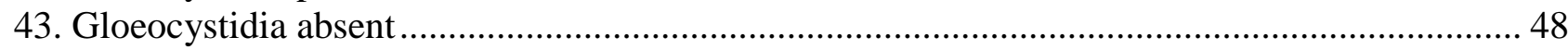

44. Context pseudoparenchymatic, hyphae cyanophilous, basidia with internal repetition.....

Conferticium

44. Not as above

45. Clamps present

Megalocystidium

45. Clamps absent. 46

46. Hyphae, basidia, and gloeocystidia amyloid

Amylofungus

46. Hyphae, basidia, and gloeocystidia not distinctly amyloid 47

47. Gloeocystidia with granular contents, SA+

Gloiothele

47. Gloeocystidia with no appreciable contents, SA-.

Vesiculomyces

48. Basidia pleural (see also Melzericium, with larger spores) Amyloxenasma

48. Basidia terminal 49

49. Lamprocystidia present. Amylostereum

49. Lamprocystidia absent. 50

50. Skeletocystidia present, hyphae simple-septate.

Aleurodiscus s.l.

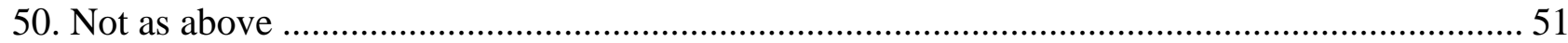

51. Basidiome effuse-reflexed to pileate, hymenophore plicate, spores very narrow, $0.5-1.2 \mu \mathrm{m}$ wide

Plicatura

51. Basidiome resupinate, hymenophore smooth to tuberculate, spores at least $1.5 \mu \mathrm{m}$ wide.......... 52

52. Spores up to $2-3 \mu \mathrm{m}$ wide, cystidia sometimes present, basidia clavate.

52. Spores larger, 3-6 $\mu \mathrm{m}$ wide, cystidia absent, basidia stalked

Amylocorticium

53. Clamps absent. .Melzericium

53. Clamps present. Hypochnella

54. Basidiospores cylindrical, brown. 54

54. Basidiospores ellipsoid, hyaline

55. Amyloid reaction weak, greyish to bluish

Corneromyces .55 Amylocorticiellum 
Key G: Basidiome usually resupinate, hymenophore non-typically poroid, without asterohyphidia; setae absent, dichohyphae and/or dextrinoid binding-skeletal hyphae absent; spores dextrinoid

1. Hymenophore smooth or tuberculate.

1. Hymenophore merulioid, reticulate, folded, hydnoid......................................................... 3

2. Cystidia present, non-septate, clamps present.... Jaapia

2. Cystidia usually absent, when present septate, clamps usually absent. Coniophora

3. Hymenophore merulioid 4

3. Hymenophore hydnoid 6

4. Hyphal system dimitic, with skeletoid hyphae, spores longer than $8 \mu \mathrm{m}$ Serpula

4. Hyphal system monomitic, spores up to $8 \mu \mathrm{m}$ long

5. Spores cylindrical, up to 1-1.5 $\mu \mathrm{m}$ wide, clamps ansiform 5

5. Spores ellipsoid, wider than 2-2.5 $\mu \mathrm{m}$, clamps not ansiform Pseudomerulius

6. Hyphae with clamps Leucogyrophana

6. Hyphae with simple septa..... Hydnomerulius

7. Spores thick-walled, hymenophore brownish with greenish tints Gyrodontium

7. Spores thin-walled, hymenophore whitish to cream Korupella

Key H: Basidiome usually resupinate, hymenophore non-typically poroid, without asterohyphidia; setae absent, dichohyphae and/or dextrinoid binding-skeletal hyphae absent; spores IKI-, basidia with 4-8 sterigmata

1. Basidia urniform 2

1. Basidia differently shaped but not urniform. 3

2. Spores thin-walled Sistotrema

2. Spores thick-walled Sistotremella

3. Basal hyphae broad, 5-10(-20) $\mu \mathrm{m}$ wide, and branched at right angles Botryobasidium

3. Basal hyphae mostly up to $5 \mu \mathrm{m}$ wide, branched at acute angles

4. Basidiome thin and hardly visible, basidia obconical to pyriform Paullicorticium

4. Basidiome distinct, basidia almost cylindric to clavate Sistotremastrum

Key I: Basidiome usually resupinate, hymenophore non-typically poroid, without asterohyphidia; setae absent, dichohyphae and/or dextrinoid binding-skeletal hyphae absent; spores IKI-, basidia with 2-4 sterigmata, spores thick-walled and smooth, clamps absent or scattered

1. Dendrohyphidia or paraphysoid hyphae present ............................................................... 2

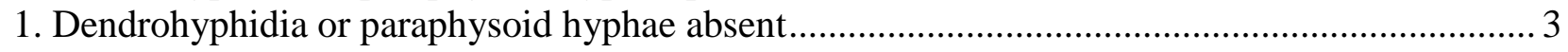

2. Hyphal system monomitic, on bark of living trees....................................................endrothele

2. Hyphae system di- to trimitic, on dead wood ............................................................. Licrostroma

3. Basidiome pinkish to red or orange, hyphae in the subiculum somewhat short-celled................ 4

3. Basidiome and subicular hyphae not as above ................................................................... 5

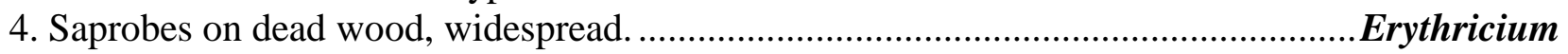

4. Parasitic or lichen-forming fungi ......................... (see also Lawreymyces) Marchandiobasidium

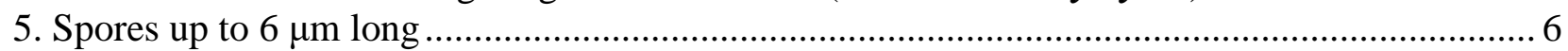

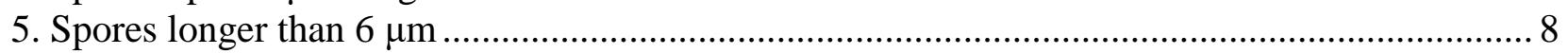

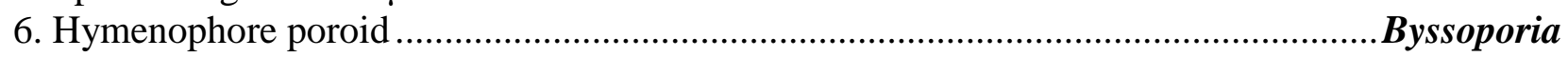

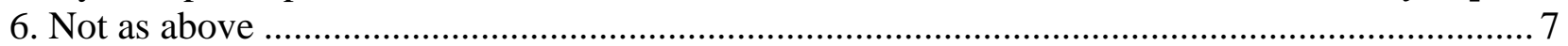

7. Basidiome bluish to greenish, some scattered clamps may be present................... Byssocorticium 
7. Basidiome white to cream to pale yellow, clamps completely absent

Piloderma

8. Spores subglobose to spherical

Membranomyces

8. Spores ellipsoid to fusoid or ovoid .... .. .9

9. Basidiome cream to brown with tuberculate to odontioid hymenophore, spores hyaline........... 10

9. Not as above, basidiome usually smooth, rarely tuberculate, spores coloured 11

10. Thin-walled cystidia present.

Phaeoradulum

10. Cystidia absent. Crepatura

11. Basidiome dark violet, spores brownish violet, slightly amyloid. Hypochnella

11. Basidiome different, spores yellowish to brownish, non-amyloid (often dextrinoid)Coniophora

Key J: Basidiome usually resupinate, hymenophore non-typically poroid, without asterohyphidia; setae absent, dichohyphae and/or dextrinoid binding-skeletal hyphae absent; spores IKI-, basidia with 2-4 sterigmata, spores thick-walled and smooth, clamps usually present (always at the basidial base)

1. Spores developing a distal rostrum when mature Arrasia

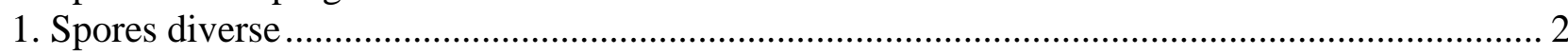

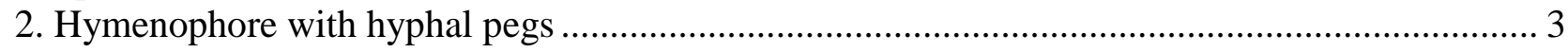

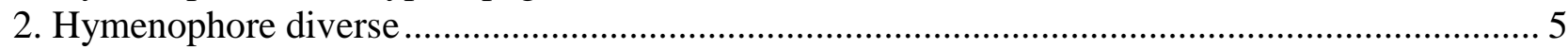

3. Hyphal pegs composed of skeletal hyphae .................................................................... Epithelopsis

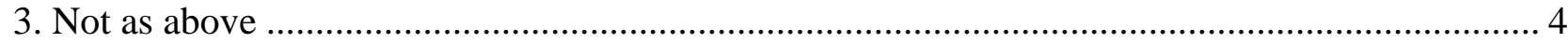

4. On dead wood, frequently on grasses ............................................ (see also Colospora) Epithele

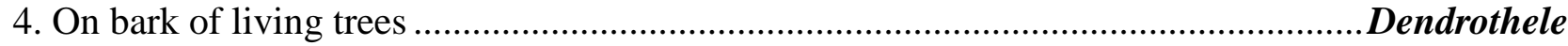

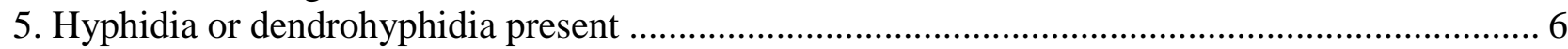

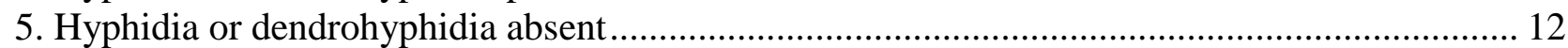

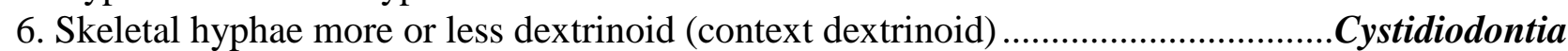

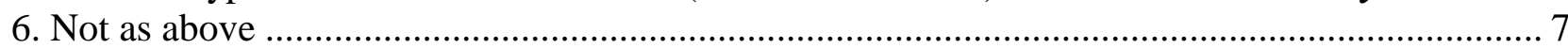

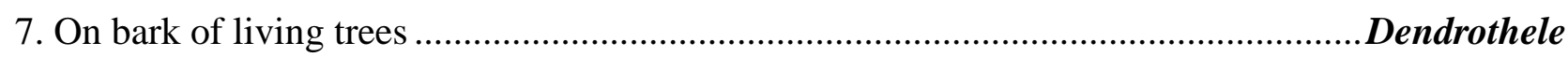

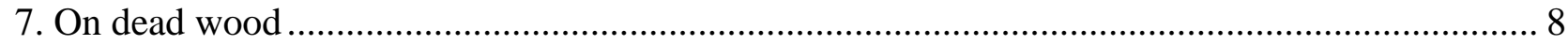

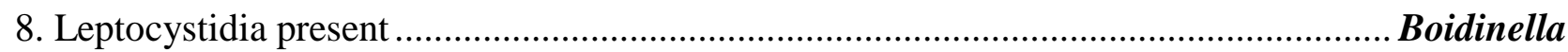

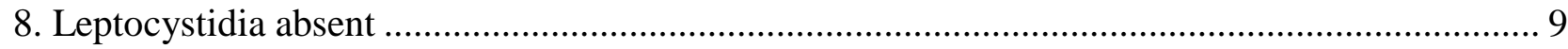

9. Basidiome ceraceous, hyphae agglutinate and indistinct, spores up to $6 \mu \mathrm{m}$ long...Dendrophlebia

9. Basidiome membranous, hyphae distinct, spores larger ...................................................... 10

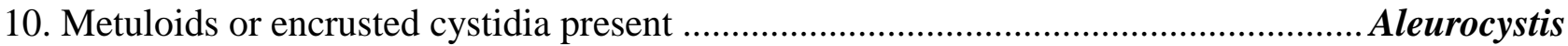

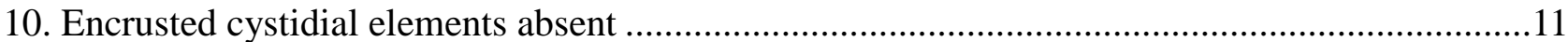

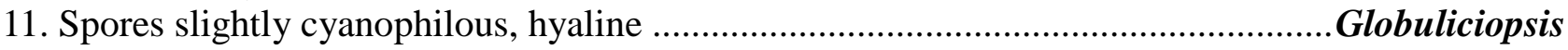

11. Spores acyanophilous, with granular contents................................................... Radulomyces

12. Thick-walled cystidia, skeletoid or hyphoid encrusted hyphae present .................................... 13

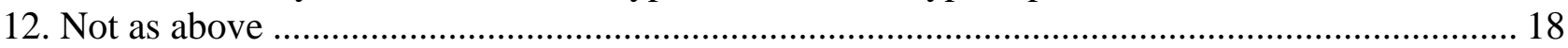

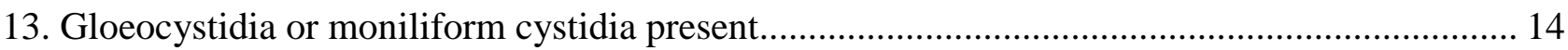

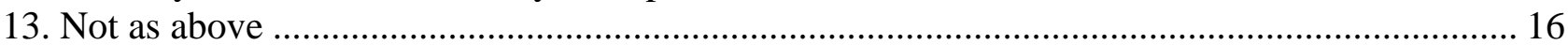

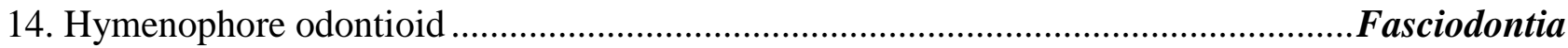

14. Hymenophore smooth to tuberculate but not odontioid ........................................................... 15

15. With brown, rooted fusiform cystidia, spores subglobose up to $6 \mu \mathrm{m}$.....................Hemmesomyces

15. With metuloids and encrusted skeletocystidia, spores ellipsoid, much larger.............. Aleurocystis

16. Hymenophore tuberculate to odontioid, with encrusted skeletoid cystidia........................ Nodotia

16. Hymenophore smooth, with hymenial or hyphoid cystidia ..................................................... 17

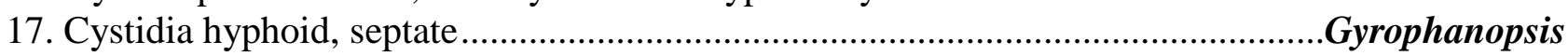

17. Cystidia hymenial, aseptate ............................................................................... Bulbillomyces

18. Hymenophore merulioid or remarkably folded, spores dextrinoid .......................................... 19

18. Hymenophore different, smooth to hydnoid, spores non-dextrinoid.......................................... 21

19. Hyphal system dimitic, spores more than $8 \mu \mathrm{m}$ long ................................................................... 
19. Hyphal system monomitic, spores up to $8 \mu \mathrm{m}$ long

20. Spores cylindrical, up to 1-1.5 $\mu \mathrm{m}$ wide, clamps ansiform

.Pseudomerulius

20. Spores ellipsoid, wider than $2-2.5 \mu \mathrm{m}$, clamps not ansiform

Leucogyrophana

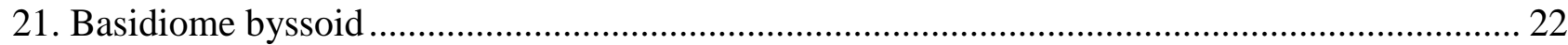

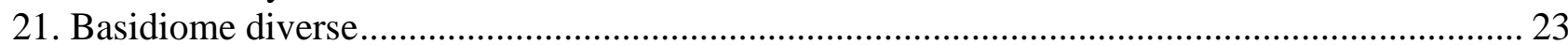

22. Basidiome with bluish to greenish colours

Byssocorticium

22. Basidiome yellowish to cream

Tretomyces

23. Basidia clearly stalked

Intextomyces

23. Basidia not stalked.

24

24. Basidia pleural

Aphanobasidium

24. Basidia terminal 25

25. Gloeocystidia moniliform and amyloid

Gloeocorticium

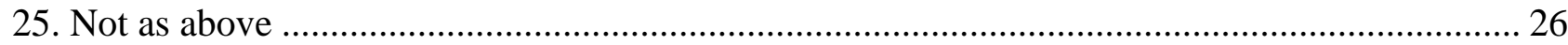

26. Some hyphae often ampullate at the septa or short-celled and inflated ................................... 27

26. Hyphae not ampullate at the septa... 28

27. Hyphae often ampullate at the septa Trechispora

27. Hyphae short-celled forming a subhymenial pseudoparenchymatous texture Hallenbergia

28. Spores up to $4-5 \mu \mathrm{m}$ long and $2-3 \mu \mathrm{m}$ wide Amphinema

28. Spores longer and/or wider. 29

29. Immature basidia with cyanophilous drops or granules Cristinia

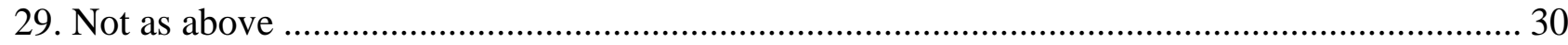

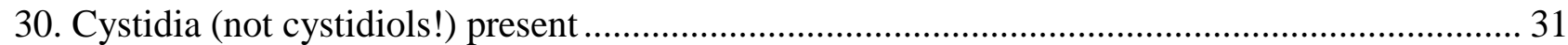

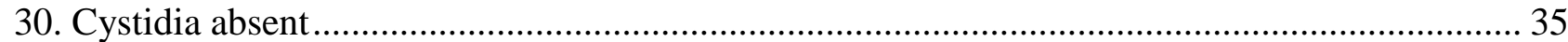

31. Hymenophore clearly hydnoid ............................................................................... Radulodon

31. Hymenophore smooth to tuberculate or odontioid .................................................................... 32

32. Cystidia (gloeocystidia) SA+ Gloeohypochnicium

32. Cystidia SA-. 33

33. Cystidia moniliform or torulose. (see also Fasciodontia) Xylodon

33. Not as above 34

34. Cystidia with obtuse apex, basidia small, 10-20 $\mu \mathrm{m}$ long, with asterocrystals.

34. Not as above (=Lagarobasidium) Xylodon Hypochnicium

35. Hymenophore distinctly odontioid to hydnoid 36

35. Hymenophore smooth to tuberculate or grandinioid 37

36. Basidiospores and subicular hyphae cyanophilous.

36. Basidiospores and hyphae non cyanophilous Cyanodontia

37. Basidia 10-15 × 3-4 $\mu \mathrm{m}$, spores $4-5 \times 2.5-3 \mu \mathrm{m}$ Sarcodontia

37. Basidia and spores wider Hypochniciellum

38. Basidia longer than $30 \mu \mathrm{m}$, spores on average more than $6 \mu \mathrm{m}$ wide Hypochnicium

38. Basidia rarely more than $30 \mu \mathrm{m}$ long, spores on average decidedly narrower

39. Hymenophore often with olivaceous tints, spores dextrinoid

39. Hymenophore bright yellow, spores non-dextrinoid

Leucogyrophana ..Coniophora

Key K: Basidiome usually resupinate, hymenophore non-typically poroid, without asterohyphidia; setae absent, dichohyphae and/or dextrinoid binding-skeletal hyphae absent; spores IKI-, basidia with 2-4 sterigmata, spores ornamented and/or branched, lobed, triangular or tetrahedral

1. Spores lobed or branched.

1. Spores variable, suballantoid, ellipsoid, fusiform, globose or with a tetrahedral outline............. 5

2. Spores Y-shaped, with two outgrowths, which in the end branch out once again.. Elaphocephala

2. Spores differently shaped 
3. Spores otherwise

4. Spores with seven lobes

Lobulicium

4. Spores more or less regularly triangular in outline, with 3 lobes

Tylospora

5. Hymenophore minutely odontioid formed by sterile hyphal pegs

5. Not as above (if hymenophore odontioid the aculei are fertile)

6. Spores biapiculate

6. Spores diverse, not typically biapiculate

7. Dendrophyses present

7. Dendrophyses absent

8. On bark of living trees

Dendrothele

8. On decayed wood

9. Hyphae simple-septate, spores more than $10 \mu \mathrm{m}$ long....

Coniophoropsis

9. Hyphae with clamps, spores up to $10 \mu \mathrm{m}$ long

Ramaricium

10. Cystidia absent (cystidiols or large subicular vesicles may be present).....

10. Cystidia present

11. Basidia with cyanophilous granular content

Lindtneria

11. Basidia without cyanophilous granular content 12

12. Hymenophore poroid Trechispora

12. Hymenophore smooth to hydnoid 13

13. Hymenophore odontioid to hydnoid 14

13. Hymenophore smooth to hypochnoid, grandinioid or tuberculate

14. Basidia clearly stalked, spores ellipsoid, slightly angular, thick-walled

14. Not as above

Intextomyces

15. Basidiospores subfusiform, yellowish, with strongly cyanophilous warts

15. Basidiospores ellipsoid to subglobose, hyaline, weakly or not cyanophilous. 15

16. Basidiome soft and fragile Kavinia

16. Basidiome membranaceous to corneous when dry 16

17. Clamps absent or inconstant Trechispora

17. Clamps present (some few basal hyphae may be simple-septate). Ripexicium

18. Basidia pleural

18. Basidia terminal Phlebiella

19. Basidia urniform to subcylindrical, hyphae broad, usually more than $5 \mu \mathrm{m}$ wide 19

19. Basidia clavate, hyphae narrow, up to $5 \mu \mathrm{m}$ wide

20. Spores thin-walled, not producing secondary spores by germination

20. Spores thick-walled, often producing secondary spores by germination

21. Spores hyaline and cyanophilous. 21

21. Not as above Tomentelloid fungi (see Key C)

22. Hyphae with ampullaceous swellings near the septa.

22. Hyphae without ampullate septa

23. Spore ornamentation developed in later stages of the maturation Trechinothus

23. Not as above Trechispora

24. Spores yellowish to pale brown, darkening to blackish brown . Scotoderma

24. Not as above .... .25

25. Basidia pleural .. 26

25. Basidia terminal .28

26. Spores angular, tetrahedral Xenosperma

26. Spores subcylindrical to ellipsoid or globose 27

27. Spores globose, slightly thick-walled, basidia large, more than $20 \mu \mathrm{m}$ long .. Cunninghammyces

27. Spores variably in shape, usually ellipsoid, basidia smaller, less than $20 \mu \mathrm{m}$ long

28. Hyphidia absent, spores rugose, cyanophilous.

28. Hyphidia present, spores minutely warted, acyanophilous Phlebiella Granulobasidium Radulomyces 


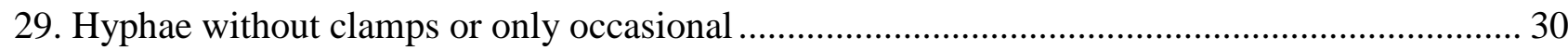

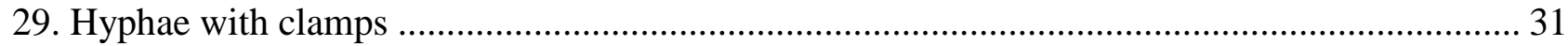

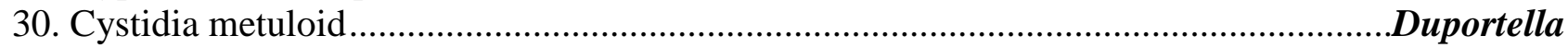

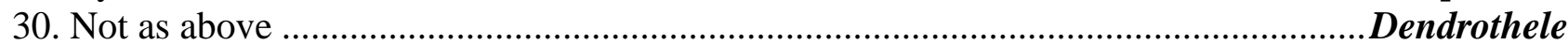

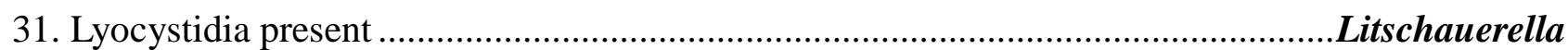

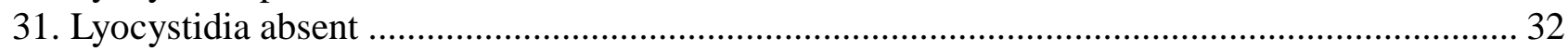

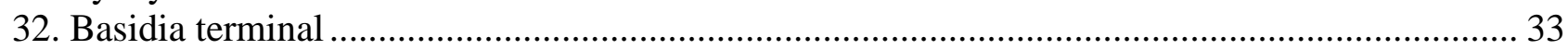

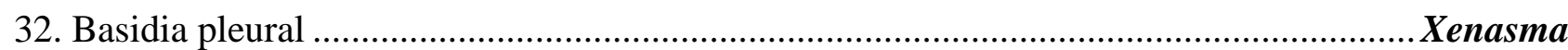

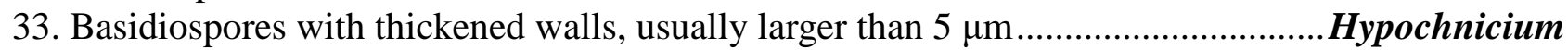

33. Basidiospores thin-walled, up to $5 \mu \mathrm{m}$ in diameter ..................................................... Rogersella

Key L: Basidiome usually resupinate, hymenophore non-typically poroid, without asterohyphidia; setae absent, dichohyphae and/or dextrinoid binding-skeletal hyphae absent; spores IKI-, basidia with 2-4 sterigmata, spores smooth, lyocystidia present

1. Hyphal system dimitic

Dacryobolus

1. Hyphal system monomitic .............................................................................................. 2

2. Hyphae without clamps .............................................................................................. Leifia

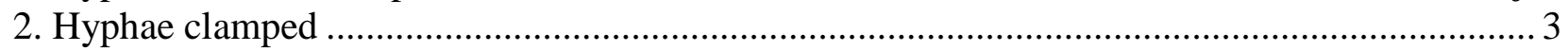

3. Hyphae and cystidia thick-walled, swelling and partly dissolving in KOH, IKI-..Amauromyces

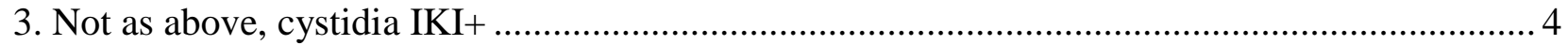

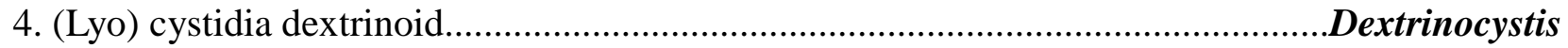

4. Lyocystidia not dextrinoid, commonly amyloid .................................................................. 5

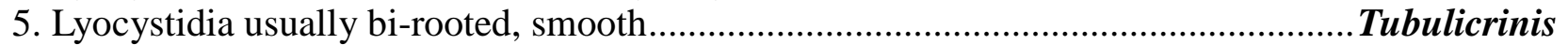

5. Lyocystidia usually multi-rooted, covered by hyphae ........................................................... 6

6. Spores sigmoid to vermiform, smooth....................................................................... Tubulicium

6. Spores subglobose, finely warted (rarely smooth) ..................................................itschauerella

Key M: Basidiome usually resupinate, hymenophore non-typically poroid, without asterohyphidia; setae absent, dichohyphae and/or dextrinoid binding-skeletal hyphae absent; spores IKI-, basidia with 2-4 sterigmata, spores smooth, lyocystidia absent, generative hyphae with simple septa, cystidial organs present

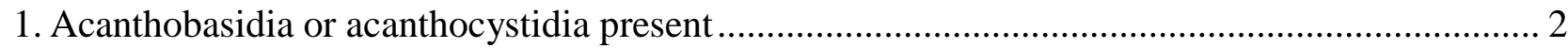

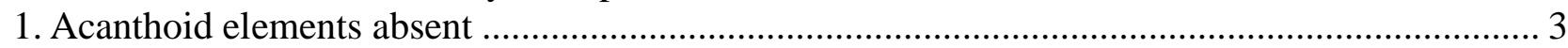

2. With acanthobasidia, not reacting in Melzer's reagent, with (1)2 long sterigmata, spores repetitive.....

Heteroacanthella

2. With acanthocystidia, dextrinoid, spores not repetitive.......................................Acanthocystidium

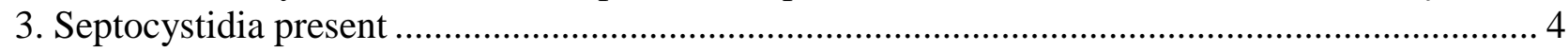

3. Clearly differentiated septocystidia absent ........................................................................... 5

4. Septocystidia not encrusted (see also Membranomyces spurius, with somewhat septate, scarcely differentiated hyphal ends).

Candelabrochaete

4. Septocystidia encrusted Odonticium

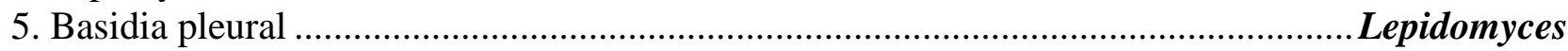

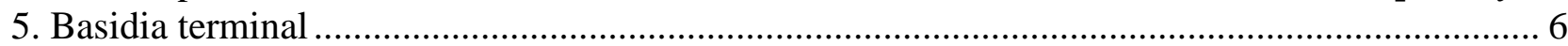

6. Cystidia of the metuloid type (thick-walled, encrusted) or heavily encrusted, thick-walled hyphal ends/pseudocystidia present .............................................................................. 7

6. Cystidia not heavily encrusted (excepting encrusted hyphal ends or encrusted tips)................. 18

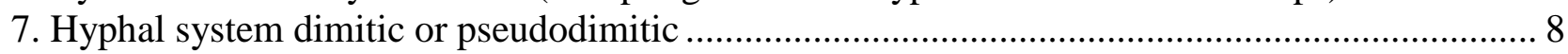

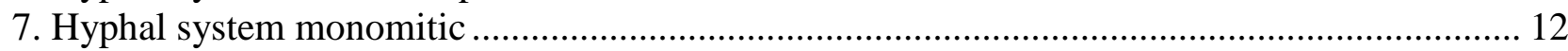

8. Hymenophore smooth to slightly tuberculate, cystidia yellowish brown to brown ......Hjortstamia

8. Hymenophore odontioid to irpicoid, cystidia hyaline .......................................................... 9 


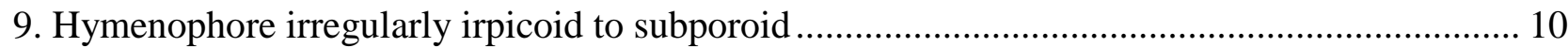

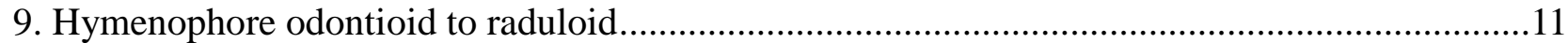

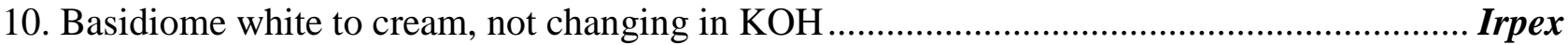

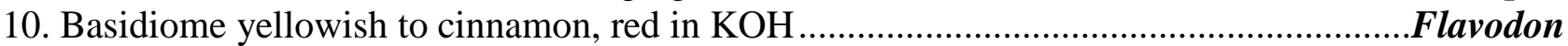

11. Hymenophore with cylindrical or conical aculei .....................................................Steccherinum

11. Hymenophore raduloid .............................................................................Australohydnum

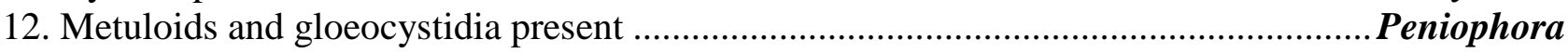

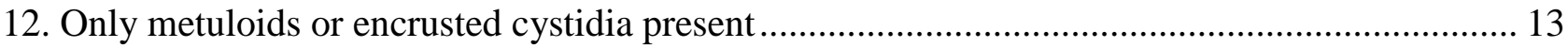

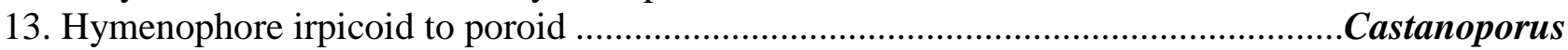

13. Hymenophore more or less smooth to grandinioid or tuberculate ......................................... 14

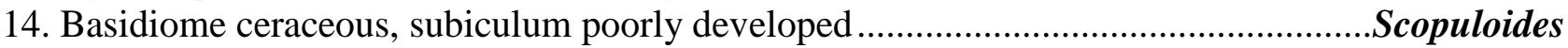

14. Basidiome subceraceous to membranaceous, subiculum well developed.................................. 15

15. Subhymenium formed by a compact structure of hyphal cords ............................... Cordochaete

15. Subhymenium not with such structure of hyphal cords........................................................ 16

16. Basidiome membranaceous, subicular hyphae in a looser structure .......................Phanerochaete

16. Basidiome subceraceous, subicular hyphae agglutinate and indistinct ................................... 17

17. Basidiome whitish to sordid, cystidia hyaline o very pale coloured ............................ Phlebiopsis

17. Basidiome beige to brown, cystidia usually brown coloured ............................. Phaeophlebiopsis

18. Basidiome pileate and with a rudimentary or more or less developed stype............................ 19

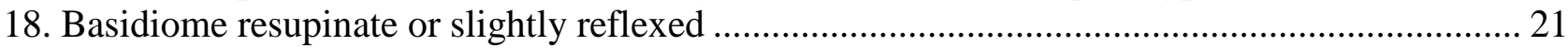

19. Basidiome stereoid, dimidiate, laterally attached to small branches ......................... Papyrodiscus

19. Basidiome flabelliform with a rudimentary stipe, on mosses or soil ........................................ 20

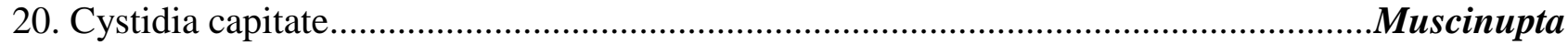

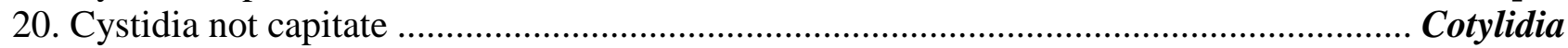

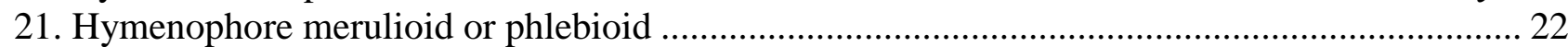

21. Hymenophore smooth to tuberculate, warted, odontioid, hydnoid or raduloid........................... 24

22. Hymenophore merulioid, cystidia thin-walled, 30-50 $\mu \mathrm{m}$ long, spores 3-6 $\mu \mathrm{m}$ long ................ 23

22. Hymenophore tuberculate to phlebioid, cystidia thick-walled 110-160 $\mu \mathrm{m}$ long, spores 6-9 $\mu \mathrm{m}$ long.

Crustoderma

23. Basidiome bluish-greyish, cystidia vesicular

Meruliophana

23. Basidiome usually creamish to reddish, cystidia diverse ....................................... Byssomerulius

24. Monomitic, hymenophore with hyphal pegs formed by tramal hyphae with walls up to $1 \mu \mathrm{m}$ thick.

Myriothele

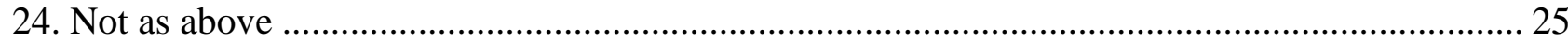

25. Hymenophore odontioid, aculei with fascicles of thin-walled, usually heavily encrusted hyphae.

26

25. Not as above, aculei not formed by heavily encrusted hyphae............................................... 27

26. Cystidia absent, only aggregated encrusted hyphal ends........................................Hyphodermella

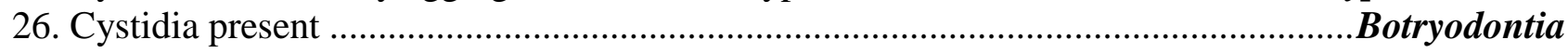

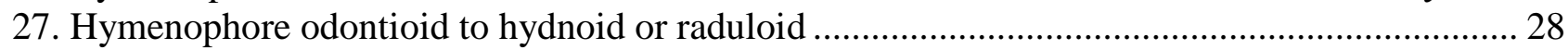

27. Hymenophore smooth to grandinioid or tuberculate ........................................................... 31

28. Basidia bisporic, cystidia thick-walled, arising deep in subiculum .....................................eiffiella

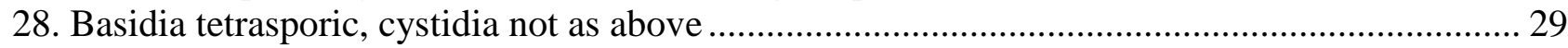

29. Basidiome ceraceous to subceraceous, hyphae difficult to discern ...................................Phlebia

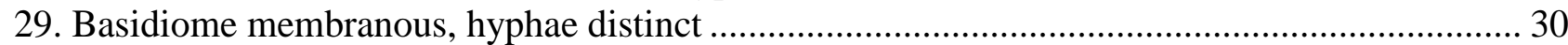

30. Margin rhizomorphic, hymenophore reddish orange yellow................................... Hydnophlebia

30. Margin not rhizomorphic, hymenophore whitish to pale yellow........................... Phanerodontia

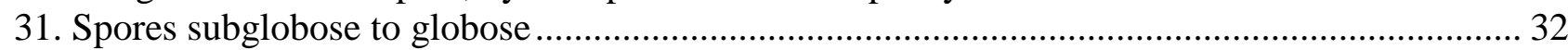

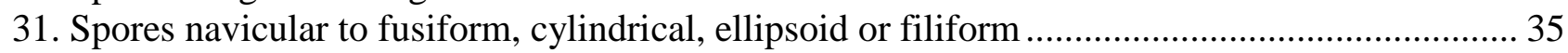

32. Cystidia poorly differentiated, hyphoid and with secondary septa.........................embranomyces

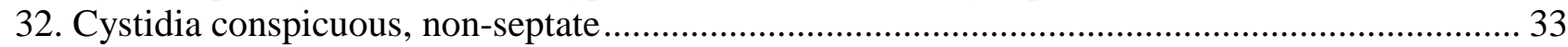

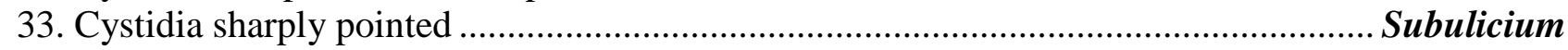


34. Cystidia with obtuse apex, basidiospores up to 6-9 $\mu \mathrm{m}$ long.....

34. Cystidia capitate, basidiospores larger, more than $9 \mu \mathrm{m}$ long

Radulomycetopsis

35. Spores repetitive

Lawrynomyces

35. Spores not repetitive

Oliveonia

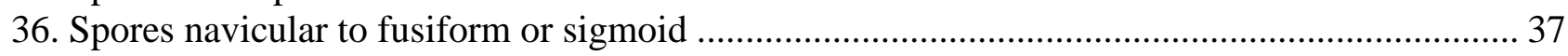

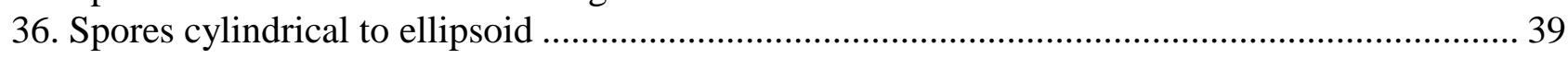

37. Hyphal system dimitic, with skeletocystidia

Veluticeps

37. Hyphal system monomitic, cystidia with a resinous encrusted apex.

38. Spores navicular, up to $8 \mu \mathrm{m}$ long, cystidia fusiform 38

38. Spores sigmoid, $10-12 \mu \mathrm{m}$ long, cystidia tubular with a capitate apex Coronicium

39. Cystidia capitate present, together with strongly encrusted hyphal ends Athelocystis

39. Not as above

Hyphoderma

40. Basidia repetitive 40

40. Basidia not repetitive

Repetobasidiopsis

41. Basidia urniform Sistotrema

41. Basidia different. .. 42

42. Basidiome athelioid; basidia short, 13-16 $\mu \mathrm{m}$ long, in clusters Athelia

42. Basidiome membranaceous or ceraceous, basidia longer, more than $25 \mu \mathrm{m}$ long, not in clusters..

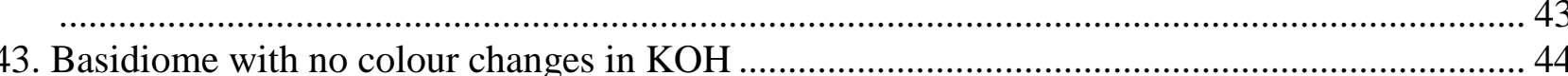

43. Hymenial surface and/or rhizomorphs reddish or purple in $\mathrm{KOH}$.......................................... 46

44. Basidiome ceraceous, hyphae usually difficult to discern ............................................... Efibulella

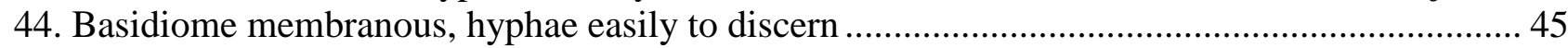

45. Subiculum distinctly brown, subicular hyphae yellowish to brown Australicium

45. Subiculum whitish, subicular hyphae hyaline

.Phanerochaete

46. Basidiospores more than $8 \mu \mathrm{m}$ long, basidia clavate to stalked clavate.

Ginnsia

46. Basidiospores smaller, up to $7 \mu \mathrm{m}$ long, basidia clavate

Rhizochaete

Key N: Basidiome usually resupinate, hymenophore non-typically poroid, without asterohyphidia; setae absent, dichohyphae and/or dextrinoid binding-skeletal hyphae absent; spores IKI-, basidia with 2-4 sterigmata, spores smooth, lyocystidia absent, generative hyphae with simple septa, cystidial organs absent (hyphidia may be present)

1. Basidiome pileate and stipitate 2

1. Basidiome resupinate.

2. Basidiome white, with a lichenized structure

.Cyphellostereum

2. Not as above Stereopsis

3. Spores repetitive

3. Spores not repetitive

4. Basidia and hyphidia with acanthoid protuberances

Heteoacanthella

4. Not as above 5

5. Sterigmata large, separated from the rest of the basidium by a simple septum. Tulasnella

5. Not as above Ceratobasidium

6. Basidia forming a regular palisade, ellipsoid to cylindrical Thanatephorus

6. Basidia not forming a regular palisade, subglobose Ceratobasidium

7. Subhymenial hyphae typically contorted, on soil or plan pathogen Waitea

7. Not as above

8. Hyphal system dimitic, skeletal hyphae with brown (green in $\mathrm{KOH}$ ) incrustations

Boreostereum

8. Hyphal system monomitic, hyphae without such incrustations.

9. Hymenophore either merulioid, grandinioid, or odontioid to hydnoid 10 
9. Hymenophore smooth to radially rugose.

10. Hymenophore odontioid, composed of sterile hyphal pegs.................................................. Epithele

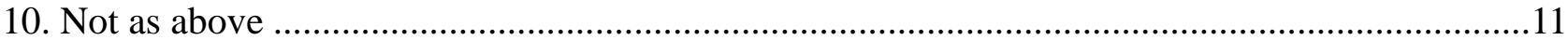

11. Basidiome soft, merulioid (in Byssomerulius pirottae, odontioid to hydnoid)........ Byssomerulius

11. Basidiome tough, grandinioid, odontioid to hydnoid ......................................................... 12

12. Hyphae in the apex of the aculei compacted and heavily encrusted .......................Hyphodermella

12. Not as above.... 13

13. Hyphae and basidial base dextrinoid

Melzerodontia

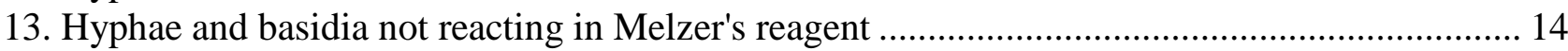

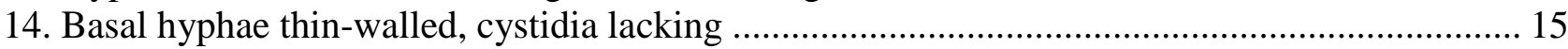

14. Basal hyphae thick-walled, hyphoid cystidiols may be present ........................................... 16

15. Hymenophore grandinioid with small aculei, rose to pinkish ............................... Roseograndinia

15. Hymenophore odontioid to hydnoid, ochraceous .........................................................Phaneroites

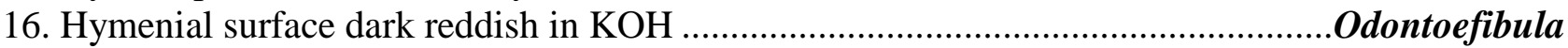

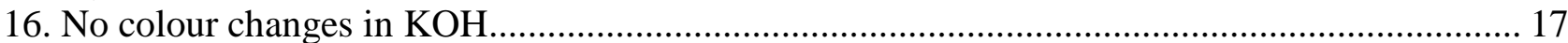

17. Quasi-binding hyphae present next to substratum.......................................Hydnophanerochaete

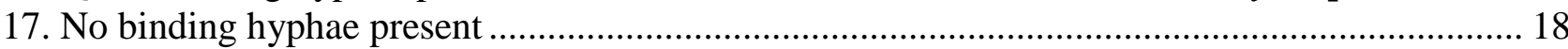

18. Hyphal system seemingly dimitic, tramal hyphae thick-walled .................................... Odonticium

18. Hyphal system monomitic, tramal hyphae usually thin-walled...............................Phanerodontia

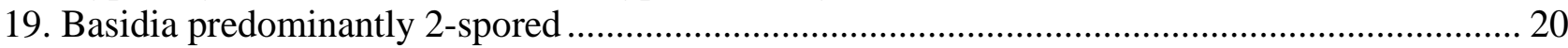

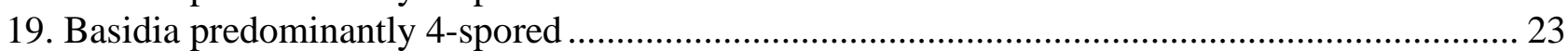

20. Basidiome brown, basidiospores fusiform to navicular ........................................................uellia

20. Basidiome differently coloured, basidiospores allantoid, ellipsoid, ovoid to pyriform ............... 21

21. Hyphidia encrusted present............................................................................... Nothocorticium

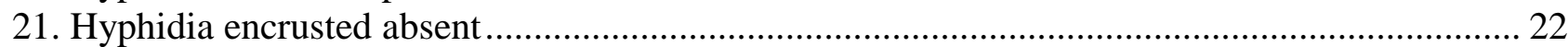

22. Basidiome orbicular, gelatinous, spores large, more than $15 \mu \mathrm{m}$ long.....................archandiopsis

22. Basidiome pellicular, spores smaller, less than $15 \mu \mathrm{m}$ long .............................................. Athelia

23. Sterigmata $10-30 \mu \mathrm{m}$ long, ab. half the length of the basidium ............................ Thanatephorus

23. Sterigmata considerably shorter, ab. $1 / 4$ of the length of the basidium...................................... 24

24. Basidia sphaeropedunculate, probasidia thin-walled, clavarioid anamorph present, pinkish

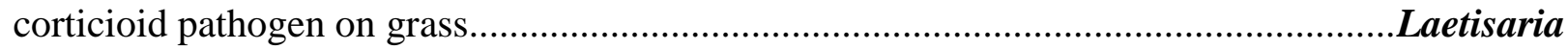

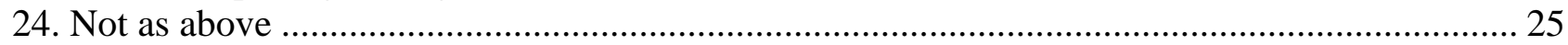

25. Basidia stalked, spores large ab. $15 \mu \mathrm{m}$ long or more ........................................................ 26

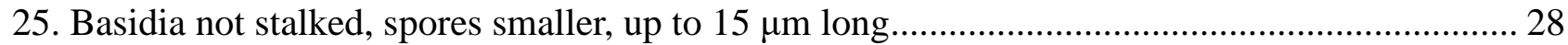

26. Spores allantoid, ab. 20-30 x 8-10 $\mu \mathrm{m}$....................................................................... Dendrominia

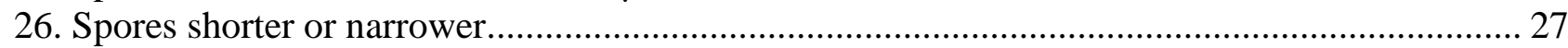

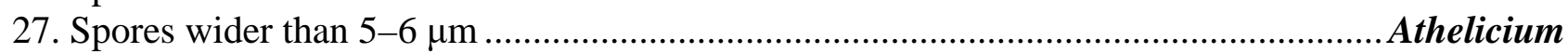

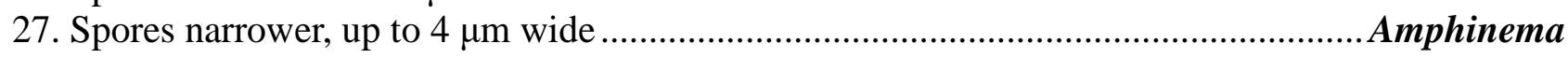

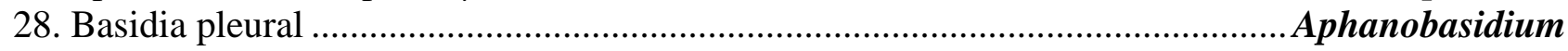

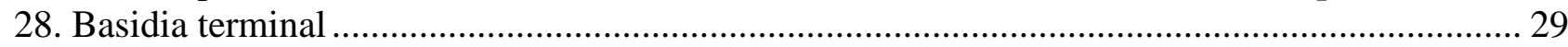

29. Basidiome ceraceous, hyphae in a compact texture .......................................................Efibula

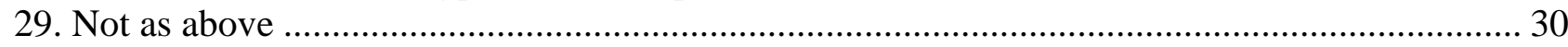

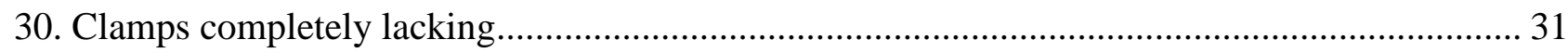

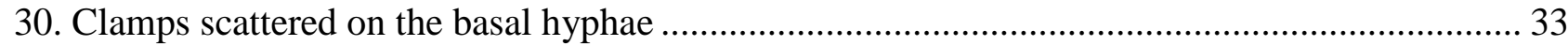

31. Basidiome yellow to orange, basidia not in candelabrum clusters ................................Athelidium

31. Basidiome whitish, basidia in candelabrum-arranged clusters .................................................. 32

32. Growing in dead wood, woody or organic debris or dead leaves....................................... Athelia

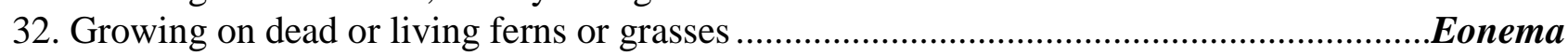

33. Basidiome athelioid to arachnoid, subiculum loose, basidia in candelabrum clusters ........ Athelia

33. Basidiome membranaceous, subiculum denser, basidia in a dense palisade............................. 34

34. Subiculum distinctly brown, subicular hyphae yellowish to brown............................Australicium

34. Subiculum whitish, subicular hyphae hyaline ........................................................Phanerochaete 
Key O: Basidiome usually resupinate, hymenophore non-typically poroid, without asterohyphidia; setae absent, dichohyphae and/or dextrinoid binding-skeletal hyphae absent; spores IKI-, basidia with 2-4 sterigmata, spores smooth, lyocystidia absent, generative hyphae with clamps, hyphal system di- or trimitic

1. Hymenophore formed by small cupuliform pores densely aggregated Porotheleum

1. Not as above

2. Basidiome stipitate with spathulate to flabelliform or infundibuliform pilei ............................. 3

2. Basidiome sessile, resupinate to effuse-reflexed or pileate

3. Hymenophore hydnoid Mycorrhaphium

3. Hymenophore smooth to rough or ribbed. 4

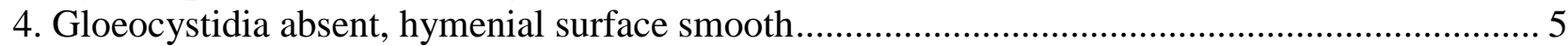

4. Gloeocystidia present, hymenial surface smooth or ribbed ...................................................... 6

5. Hyphal system trimitic, binding hyphae present Aquascypha

5. Hyphal system dimitic, with skeletal hyphal restricted to the stipe Inflatostereum

6. Hymenial surface ribbed, abhymenial surface hirsute or tomentose Cymatoderma

6. Hymenial surface smooth, abhymenial surface glabrous Podoscypha

7. Skeletal hyphae dextrinoid (different from the binding-skeletal hyphae of Scytinostroma)......... 8

7. Skeletal hyphae non-dextrinoid.... 9

8. Hymenophore merulioid, leptocystidia present, spores fusiform Merulicium

8. Hymenophore hydnoid, cystidia absent, spores subglobose

(see also Trechispora) Dextrinodontia

9. Cystidia and dendrohyphidia absent 10

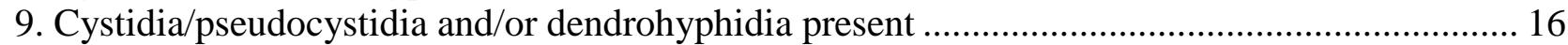

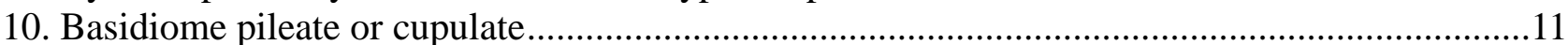

10. Basidiome resupinate.

11. Basidiome cupulate, skeletoid hyphae close to the substrate Auriculariopsis

11. Basidiome pileate, dimitic hyphal system with arboriform hyphae Mycobonia

12. Hyphae often ampullate at the septa, hymenophore arachnoid to granulose Trechispora

12. Hyphae not ampullate at the septa, hymenophore odontioid to hydnoid

13. Micro-binding hyphae present Phlebia

13. Only skeletal hyphae present.

14. Basidia more than $25 \mu \mathrm{m}$ long.

14. Basidia smaller, up to $20 \mu \mathrm{m}$ long .....

Fibricium

15. Basidia up to $10 \mu \mathrm{m}$ long, with smooth skeletal hyphae

15. Basidia 10-20 $\mu \mathrm{m}$ long, usually with encrusted (pseudo)skeletal hyphae Ceraceohydnum

16. Hymenophore odontioid to hydnoid Fibrodontia

16. Hymenophore smooth to tuberculate $\ldots$

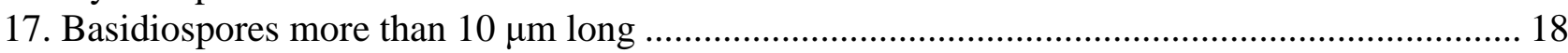

17. Basidiospores up to $10 \mu \mathrm{m}$ long

18. Hymenophore formed by hyphal pegs fused laterally in a shallow semireticulum, basidiospores more than $25 \mu \mathrm{m}$ long

Pileodon

18. Hymenophore with isolated hyphal pegs (not fused), basidiospores up to $20 \mu \mathrm{m}$ long.. Veluticeps

19. Encrusted skeletocystidia present..... Steccherinum

19. Encrusted skeletocystidia absent .... 20

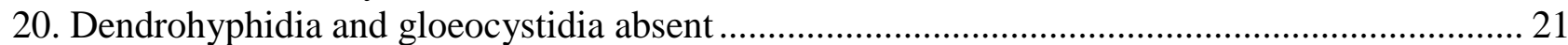

20. Dendrohyphidia and/or gloeocystidia present .................................................................... 23

21. Tramal and/or hymenial cystidia and microbinding hyphae present ...........Pseudolagarobasidium

21. Not as above 22

22. Basidiospores more than $4.5 \mu \mathrm{m}$ long, cystidia with an apical resinous cap.

22. Basidiospores up to 3.5-4 $\mu \mathrm{m}$ long, cystidiols fusiform .... Mycoaciella

23. Gloeocystidia or oleiferous hyphae present.... Ceraceohydnum 24 
23. Gloeocystidia or oleiferous hyphae absent

24. Dendrohyphidia and vesicular to ovate gloeocystidia present

Crustomyces

24. Paraphysate hyphae present, with tubular oleiferous hyphae

Stecchericium

25. Hymenophore formed by sterile hyphal pegs of straight skeletal hyphae

Skeletohydnum

25. Hymenophore odontioid, skeletals branched restricted to the subiculum

Dendrodontia

26. With brown skeletal hyphae, hyaline dendrohyphidia and pyriform basidiospores

(see also Neocampanella for similar species without skeletal hyphae) Brunneocorticium

26. Not as above....

27

27. Hyphal system pseudodimitic, brown dendrohyphydia present

Dendrophora

27. Not as above, brown dendrohyphidia absent

28

28. Skeleto-binding or micro-binding hyphae present

29

28. Only skeletal hyphae present

29. Skeletal, binding hyphae and dendrohyphidia present

Trimitiella

29. Not as above

30

30. Basidiome (when fresh) reddish in $\mathrm{KOH}$ (hyphae and crystals in $\mathrm{KOH}$ reddish)......Crustodontia

30. Basidiome not changing in $\mathrm{KOH}$.

31. Basidiome bluish to violet, cystidia absent.

Amethicium

31. Not with this combination of characters

32

32. Cystidia basally encrusted with resinous yellowish matter

Cericium

32. Not as above

Phlebia

33. Dark brown pseudocystidia and/or metuloids present

34

33. Dark brown pseudocystidia and metuloids absent (hyaline to pale yellow brown metuloids may be present)..... 36

34. Gloeocystidia present.

Duportella

34. Gloeocystidia absent 35

35. Spores more than $10 \mu \mathrm{m}$ long Veluticeps

35. Spores up to $6-8 \mu \mathrm{m}$ long... Porostereum

36. Skeletocystidia present, spores usually more than $10 \mu \mathrm{m}$ long. Lopharia

36. Not with the previous combination of characters 37

37. Skeletal hyphae with cyanophilous walls, spores subfusiform Minostroscyta

37. Not as above .. 38

38. Spores $8-10 \times 4.5-5 \mu \mathrm{m}$, skeletals rare

Hyphoderma

38. Spores up to $7 \mu \mathrm{m}$ long, skeletals conspicuous 39

39. Basidiome crustaceous, stratified, vesicular gloeocystidia abundant

39. Basidiome membranaceous to waxy, gloeocystidia few or absent

Cystostereum

Fibricium

Key P: Basidiome usually resupinate, hymenophore non-typically poroid, without asterohyphidia; setae absent, dichohyphae and/or dextrinoid binding-skeletal hyphae absent; spores IKI-, basidia with 2-4 sterigmata, spores smooth, lyocystidia absent, generative hyphae with clamps, hyphal system monomitic, dendrohyphidia present

1. Basidiome iridescent bluish

Terana

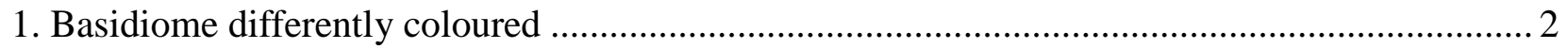

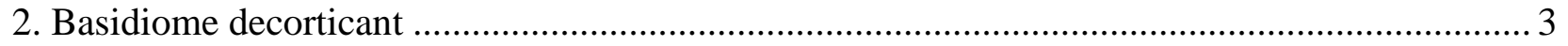

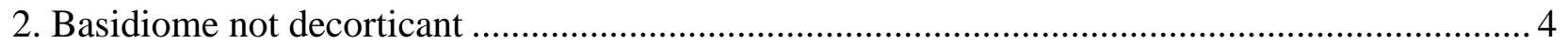

3. Basidial layer developed in a hymenium and subiculum distinguishable ..... Australovuilleminia

3. Basidia not in a distinguishable hymenium and subiculum indistinguishable

..(see also Dendrominia) Vuilleminia

4. On bark of living trees, spores cyanophilous (but often difficult to verify!). Dendrothele

4. Not as above 5

5. Repetobasidia present

Repetobasidiellum

5. Repetobasidia absent or only occasional 6 


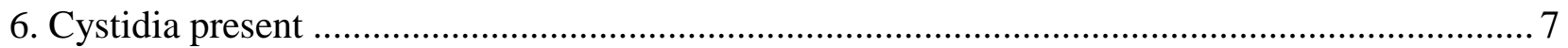

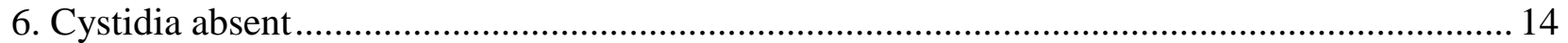

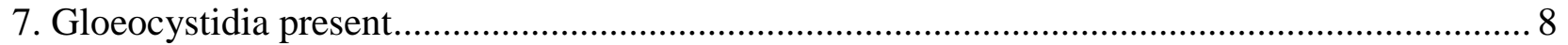

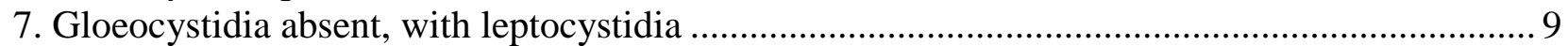

8. Dendrohyphidia soon brown coloured................................................................ Dendrophora

8. Dendrohyphidia remaining hyaline or darkening slightly with age ...........................Peniophora

9. Leptocystidia subulate, spores fusiform to navicular ........................................... Leptocorticium

9. Leptocystidia with capitate, obtuse apex or fusoid apex, spores otherwise ............................. 10

10. Leptocystidia with obtuse apex, spores subcylindrical up to $6-8 \mu \mathrm{m}$ long ................ Crustomyces

10. Leptocystidia capitate or subfusiform, spores diversely shaped ............................................. 11

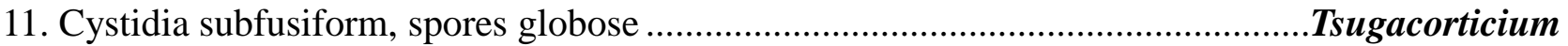

11. Cystidia capitate, spores globose, pyriform or allantoid ....................................................... 12

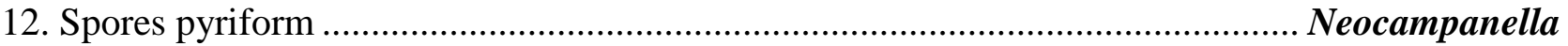

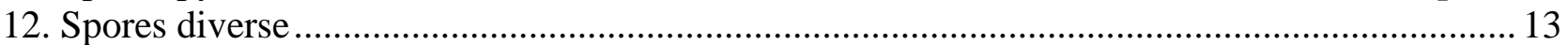

13. Basidiome with a shade of brown, spores subglobose ...................................... Punctulariopsis

13. Basidiome light coloured, whitish, spores allantoid................................................ Vuilleminia

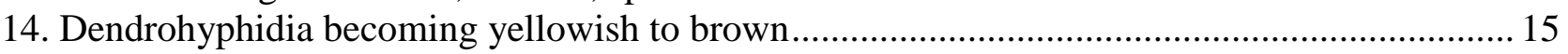

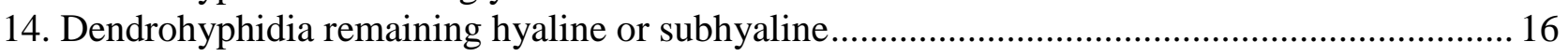

15. Hymenium bright red to violaceous red, spores more than $10 \mu \mathrm{m}$ long......................................ia

15. Hymenium brown to dark violaceous, spores up to $9 \mu \mathrm{m}$ long .................................. Punctularia

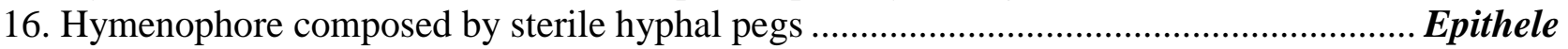

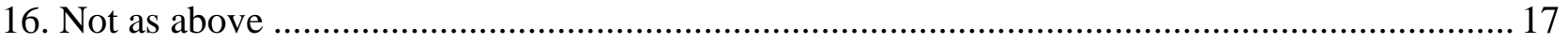

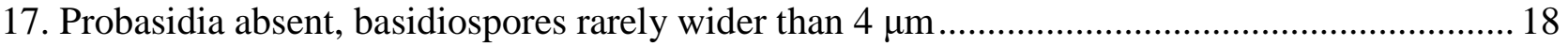

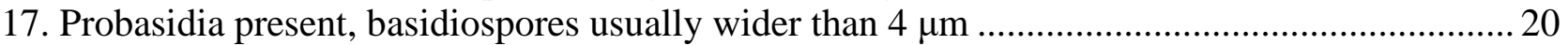

18. Skeletal (pseudoskeletal) brown hyphae present in context ................................... Dendrodontia

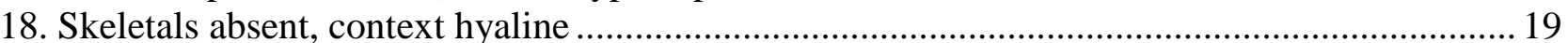

19. Basidia long clavate, longer than $30 \mu \mathrm{m}$, dendrophyses patent.......................... Dentocorticium

19. Basidia utriform, usually up to $30 \mu \mathrm{m}$ long, dendrophyses not conspicuous ............. Bourdotiella

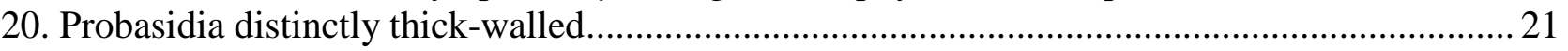

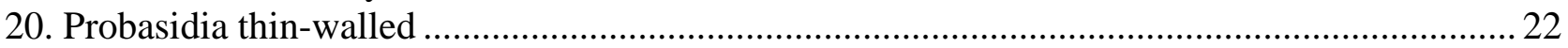

21. Basidial walls amyloid, especially where thickened .......................................... Amylobasidium

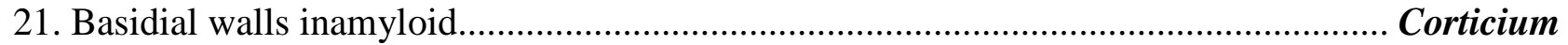

22. Basidiome with a gelatinous consistency when fresh...................................... Punctulariopsis

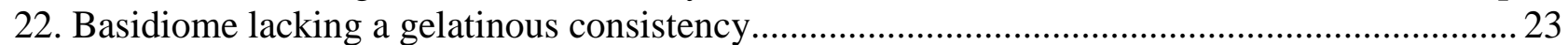

23. Spores globose to ellipsoid or ovoid...................................................................endrocorticium

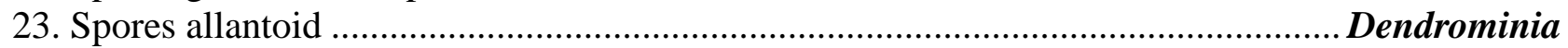

Key Q: Basidiome usually resupinate, hymenophore non-typically poroid, without asterohyphidia; setae absent, dichohyphae and/or dextrinoid binding-skeletal hyphae absent; spores IKI-, basidia with 2-4 sterigmata, spores smooth, lyocystidia absent, generative hyphae with clamps, hyphal system monomitic, dendrohyphidia absent, cystidia present

1. Hyphae and cystidia amyloid

Amylohyphus

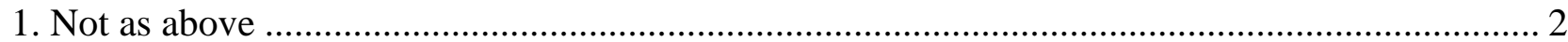

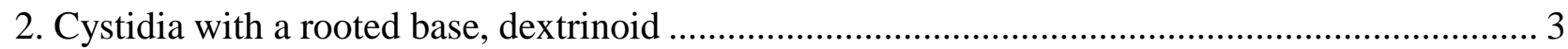

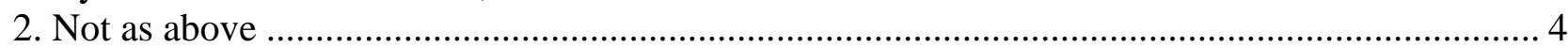

3. Cystidia with an apical umbrella-like encrustation, spores thin-walled ..................... extrinocystis

3. Cystidia lightly encrusted, not with an apically cap, spores slightly thick-walled Hemmesomyces

4. Subicular hyphae with projections covered with a double cap encrustation ................ Sceptrulum

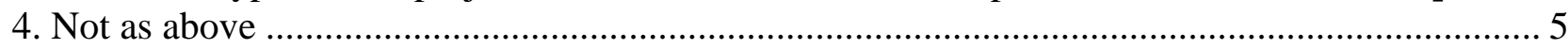

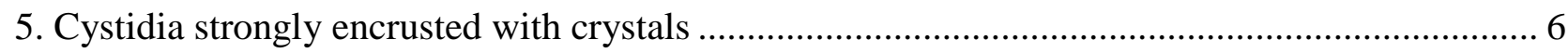

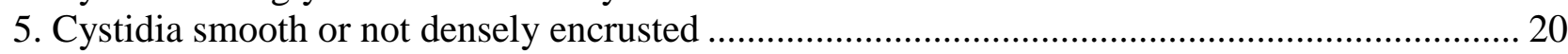


6. Basidiome red to brown, (pseudo)cystidia with a shade of brown........................................ 7

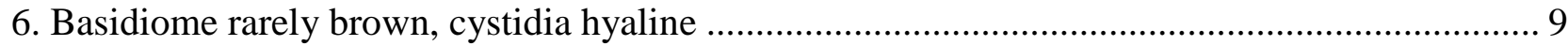

7. Basidiome reddish, strongly pilose by the thick-walled protruding cystidia, wood discoloured reddish by the fungus

Erythromyces

7. Not as above

8. Basidiome cupulate, gregarious

Campylomyces

8. Basidiome resupinate to effuse-reflexed Duportella

9. Hymenophore distinctly odontioid to raduloid or hydnoid

9. Hymenophore smooth to grandinioid or tuberculate

10. Basidiome decorticant, gloeocystidia present. Peniophora

10. Basidiome not decorticant, gloeocystidia absent. (see also Cabalodontia) Phlebia

11. Basidiome semigelatinous, hyphae gelatinized and difficult to discern

11. With a different combination of characters.... Phlebia

12. Hymenophore with rounded tubercles

12. Hymenophore smooth to grandinioid

Scopulodontia

13. Basidiome pink, orange, reddish, dark bluish or violaceous 13

13. Basidiome differently coloured Peniophora

14. Cystidia subulate, characteristically encrusted with rectangular crystals. 14

14. Cystidia different Subulicystidium

15. Cystidia cylindrical to subulate, encrusted only at the basal part, apically naked, basidia mostly pleural.

Lepidomyces

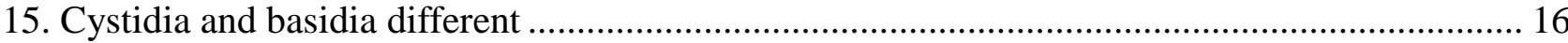

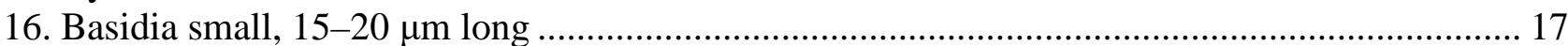

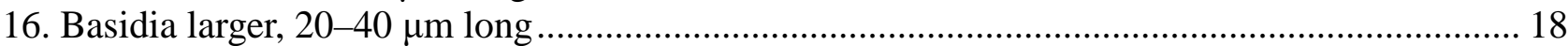

17. Gloeocystidia SA+ present, leptocystidia absent.

Metulodontia

17. Gloeocystidia SA+ absent, leptocystidia cylindrical to capitate present (=Palifer) Xylodon

18. Spores up to $6 \mu \mathrm{m}$ long, basal hyphae $4-8 \mu \mathrm{m}$ wide Ceraceomyces

18. Spores on average more than $7 \mu \mathrm{m}$ long, basal hyphae $2-4 \mu \mathrm{m}$ wide 19

19. Basidia clavate to subcylindrical, echinulate cells usually present Peniophorella

19. Basidia suburniform, echinulate cells absent. Hyphoderma

20. Septate cystidia present. 21

20. Septate cystidia lacking 29

21 . Spores $12-18 \mu \mathrm{m}$ long, fusiform to narrowly fusiform

21. Spores different

Suillosporium

22. Repetobasidia present

Repetobasidium

22. Basidia not repetitive 23

23. With lagenocystidia Hyphodontia

23. Without lagenocystidia 24

24. Septocystidia hyphoid, very finely encrusted with thin crystals Amphinema

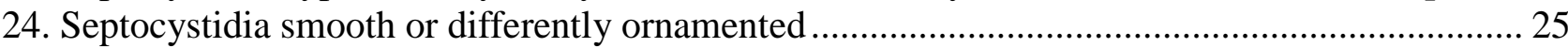

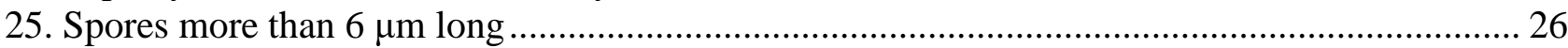

25. Spores up to $4.5 \mu \mathrm{m}$ long 27

26. Basidiome ceraceous, cystidia with several septa, more or less encrusted

26. Basidiome pellicular, cystidia with one clamped septum, smooth

Hyphoderma

27. Cystidia and basidia thin-walled, cystidia rare

Atheloderma

27. Cystidia and basidia basally thick-walled, cystidia abundant Ceraceomyces

28. Cystidia smooth faintly cyanophilous

28. Cystidia smooth or some encrusted as lamprocystidia, acyanophilous .. 28

29. Basidiome pileate, hymenophore hydnoid Tubulicrinopsis Amauromyces

29. With a different combination of characters. 30

30. Clamps few and scattered in some hyphae but absent in the basidial base 32

30. All hyphae and basidial base with clamp-connections

Climacodon 31 
31. Oleiferous hyphae present Stecchericium

31. Oleiferous hyphae absent

32. Basidiome resupinate to effuse-reflexed, hymenophore smooth, violaceous .Chondrostereum

32. Not as above 33

33. Cystidia tubular and thick-walled, at least towards the base 34

33. Cystidia different 38

34. Spores on average less than $7 \mu \mathrm{m}$ long....

34. Spores on average more than $7 \mu \mathrm{m}$ long Kneiffiella

35. Spores up to1.5-2 $\mu \mathrm{m}$ wide 35

35. Spores wider than $1.5-2 \mu \mathrm{m}$ 36

36. Cystidia 8-12 $\mu \mathrm{m}$ wide, with a drop of secretion at the subcapitate apex 37

36. Cystidia 5-6 $\mu \mathrm{m}$ wide, without drop of secretion at the apex

37. Cystidia 150-300 $\mu \mathrm{m}$ long, basidia 50-100 $\mu \mathrm{m}$ long

37. Cystidia up to $100-130 \mu \mathrm{m}$ long, basidia up to $20-50 \mu \mathrm{m}$ long

38. Hymenophore distinctly poroid .

38. Hymenophore not poroid (may be subporoid in some areas or in the margin) (=Chaetoporellus) Kneiffiella

39. Basidiome gelatinous to ceraceous, hyphae gelatinized difficult to discern 39

39. Basidiome not ceraceous, hyphae usually distinct 40

40. Sterigmata large, separated from the rest of the basidium by a simple septum, spores repetitive ..

Tulasnella

40. Not as above

41. Hymenophore reddish in $\mathrm{KOH}$

Crustodontia

41. Hymenophore with no colour changes in $\mathrm{KOH}$ Phlebia

42. Halocystidia present (capitate cystidia with a more or less conspicuous halo). 43

42. Halocystidia absent

43. Asterocystidia present

43. Asterocystidia absent Resinicium

44. Hymenophore odontioid to raduloid or hydnoid (check also Resinicium s.l.) Skvortzovia

44. Hymenophore more or less smooth or merulioid to phlebioid 45

45. Rosette-like crystals present, cystidiols capitulate, spores allantoid Sidera

45. Not as above, rosette-like crystals absent. 46

46. Cystidia excreting a drop of sticky liquid, spores allantoid

46. Cystidia not as above, spores variable

Dacryobolus

47. Spores $8-12 \mu \mathrm{m}$ long. 47

47. Spores shorter

Basidioradulum

48. With subulate leptocystidia and gloeocystidia, spores 3-4 $\mu \mathrm{m}$ long .48

48. Not as above Physodontia

49. Gloeocystidia distinct 49

49. Gloeocystidia absent or indistinct. .50

50. Gloeocystidia with yellowish contents, brownish in sulphovanillin 51

50. Gloeocystidia encrusted with yellowish resinous matter, negative in sulphovanillin.Parvodontia

51. With thin-walled tubular cystidia, spores allantoid .(=Alutaceodontia) Kneiffiella

51. Spores and/or cystidia different 52

52. Basidiome yellowish to brown or orange, hyphae usually coloured..........Pseudolagarobasidium

52. Not as above

53. With large, distinct, and projecting capitate leptocystidia 53

53. Cystidia or cystidioles not distinct, as capitate hyphal ends (torulose cystidia or gloeocystidia may be present but rare or inconspicuous). Xylodon

54. Spores repetitive, basidia with comparatively very long sterigmata .......................................... 55

54. Spores not repetitive, basidia with shorth sterigmata .......................................................... 56

55. Acanthophyses (and acanthobasidia) present, basidia with one long sterigmata Heteroacanthella

55. Acanthophyses absent, basidia usually with 2-4 sterigmata Oliveonia 
56. Basidiome thin and inconspicuous, basidia subglobose to obconical

Sphaerobasidium

56. Basidiome distinct and conspicuous, basidia not as above.

57. Cystidia with an apical papilla or a cap of yellow to reddish-brown incrustations.......Coronicium

57. Not as above ....

58. Basidiome pellicular, capitate cystidia present Lyoathelia

58. Not as above 59

59. Spores on average more than $8 \mu \mathrm{m}$ long. 60

59. Spores on average less than $8 \mu \mathrm{m}$ long.... 63

60. Basidia suburniform. .Hyphoderma

60. Basidia clavate 61

61. Spores dacryoid to obliquely ellipsoid, often in groups of 2-4

61. Spores not as above Cylindrobasidium

62. Spores $6.5-8 \mu \mathrm{m}$ wide, echinulate cells absent 62

62. Spores up to $5 \mu \mathrm{m}$ wide, echinulate cells usually present Clavulicium

63. Basidia urniform to suburniform ... Peniophorella

63. Basidia otherwise 64

64. Basidia urniform, cystidia present as flexuose gloeocystidia 68

64. Basidia suburniform, cystidia different Sistotrema

65. Cystidia 6-13 $\mu \mathrm{m}$ wide and often longer than $100 \mu \mathrm{m}$. 65

65. Cystidia only 3-7 $\mu \mathrm{m}$ wide and generally up to $60 \mu \mathrm{m}$ long. 66

66 . With two kinds of cystidia:1) moniliform and 2) subulate or capitate

66. Not as above Hastodontia

67. Capitate or subulate, more or less encrusted cystidia present 67

67. Cystidia, if present, different, not distinct, as hyphoid cystidiols

68. Basidia with a basal clamp, subicular hyphae with simple-septa.

68. Basidia and all hyphae with clamps.

...Hyphoderma

69. Basidia 8-12 $\mu \mathrm{m}$ long, with clavate to flexuous gloeocystidia

69. Basidia larger, gloeocystidia absent. Lyomyces ..Xylodon

70. Hymenophore becoming violaceous in $\mathrm{KOH}$.

70. Not as above

71. Spores $3-5 \mu \mathrm{m}$ wide, often glued in groups of $2-4$.

71. Spores up to $3 \mu \mathrm{m}$ wide Hyphodontiella ..................... 69 Parvobasidium Ceraceomyces 70 71

Key R: Basidiome usually resupinate, hymenophore non-typically poroid, without asterohyphidia; setae absent, dichohyphae and/or dextrinoid binding-skeletal hyphae absent; spores IKI-, basidia with 2-4 sterigmata, spores smooth, lyocystidia absent, generative hyphae with clamps, hyphal system monomitic, dendrohyphidia absent, cystidia absent

1. Basidiome cup-shaped or disciform

1. Basidiome not as above

2. Hyphal system dimitic/pseudodimitic, hymenophore smooth to folded Auriculariopsis

2. Hyphal system monomitic, hymenophore hydnoid by protruding hyphal fascicles

3. Basidiome gregarious formed by minute globules Mycothele

3. Basidiome not as above Mycostigma

4. Basidiome with well-developed pilei and a rudimentary to distinct stipe 4

4. Basidiome resupinate or slightly reflexed 5

5. Basidiome flabelliform to tubaeform, hymenophore smooth to rough .................................... 6

5. Basidiome dimidiate, with a stipe more or less developed, hymenophore aculeate or meruloid.. 8

6. Basidiome tubaeform with a hollow stipe Parastereopsis

6. Basidiome flabelliform, stipe if present solid. Stereopsis

7. Skeletal hyphae absent, hyphae non-inflated.

7. Skeletal hyphae present in the stipe, contextual hyphae inflated Inflatostereum 
8. Basidiome with well-developed pilei, hymenophore merulioid

8. Basidiome stipitate-pileate, hymenophore aculeate

Mycorrhaphium

9. Sterigmata large, separated from the rest of the basidium by a simple septum, spores repetitive ..

Tulasnella

9. Not as above

10. Basidiome pinkish, pathogens on grasses (Gramineae)

Limonomyces

10. Not as above, fungi usually growing on wood

11. Hymenophore tuberculate, odontioid to raduloid, phlebioid, merulioid or poroid.....

11. Hymenophore smooth to farinaceous

12. Hymenophore poroid, basidia urniform

Sistotrema

12. Hymenophore and basidia different. 13

13. Hymenophore merulioid or phlebioid 14

13. Hymenophore distinctly tuberculate to odontioid, hydnoid or raduloid.

14. Basidiome subgelatinous to ceraceous, hyphae embedded in a gelatinous matrix, clamps and hyphae difficult to discern.....

Phlebia

14. Basidiome not ceraceous, subiculum membranaceous, hyphae distinct and easily to discern.... 15

15. Hymenophore smooth to slightly merulioid, spores subglobose to ellipsoid.

15. Hymenophore distinctly merulioid, spores narrowly cylindrical to fusiform

Ceraceomyces

16. Hyphae in subiculum and cords with ampullaceous swellings near the septa Serpulomyces

16. Hyphae with no ampullate swelling (but isodiametric hyphae may be present) 17

17. Spores $8-10 \mu \mathrm{m}$ long

17. Spores shorter, up to $5 \mu \mathrm{m}$ long....

Hydnocristella

18. Hymenophore with sterile hyphal pegs Trechispora

18. Not as above

19. Spores usually longer than $10 \mu \mathrm{m}$, basidia large, normally longer than $50 \mu \mathrm{m}$

19. Spores smaller up to $10 \mu \mathrm{m}$, basidia about $10-15 \mu \mathrm{m}$ long.

20. Hyphal system pseudodimitic, with skeletoid or pseudoskeletal hyphae. Epithele

20. Hyphal system monomitic Pteridomyces

21. Basidia suburniform (cystidiols often present!) Fibrodontia

21. Basidia different Xylodon

22. Subicular hyphae thick-walled, refractive or pigmented

22. Not as above 22

23. Subicular hyphae hyaline, refractive in $\mathrm{KOH}$. 23 24

23. Subicular hyphae brownish, non-refractive in $\mathrm{KOH}$

24. Subhymenial hyphae isodiametric....

24. Subhymenial hyphae not isodiametric

25. Spores ellipsoid to globose or more or less rhomboid

25. Spores reniform to allantoid

26. Hymenophore hydnoid to raduloid. 21

26. Hymenophore tuberculate to odontioid.... Odontiopsis

27. Hymenophore raduloid, spores ellipsoid to globose 8-12 $\mu \mathrm{m}$ long

27. Hymenophore hydnoid, spores up to $8 \mu \mathrm{m}$ long

28. Spores ellipsoid

28. Spores pyriform

29. Basidia terminal, spores ellipsoid to allantoid.

29. Basidia pleural, spores globose.

30. Spores repetitive patura) Pirex

30. Spores not repetitive .. 25 26

31. Basidia clavate to ovoid, spores citriform, if subglobose to ellipsoid then $2-4 \mu \mathrm{m}$ wideOliveonia

31. Basidia obconical, spores subglobose to ovoid 3.5-6 $\mu \mathrm{m}$ wide Scotomyces

32. Basidia frequently pleural. 33

32. Basidia terminal, only occasionally pleural 34 
33. Basidia 30-50 $\mu \mathrm{m}$ long, with a lateral hook-like hypha....

Uncobasidium

33. Basidia shorter, without such a lateral hook-like hypha....

Aphanobasidium

34. Basidia urniform, hyphae characteristically with oily contents.

Sistotrema

34. Basidia and/or hyphae differently characterized 35

35. Hyphae in subiculum and cords often ampullate near the septa

Trechispora

35. Hyphae in subiculum and cords different (ampullaceous swellings may occur in more or less isodiametric hyphae in subhymenium)

36. Subhymenial hyphae short-celled, more or less isodiametric and swollen 37

36. Subhymenial hyphae long celled, not isodiametric 38

37. Spores $8-10 \mu \mathrm{m}$ long

37. Spores up to $6 \mu \mathrm{m}$ long

Conohypha

38. Hyphae alternating clamps and simple septa

38. All septa with clamps.

Brevicellicium

39. Spores yellowish to ochraceous coloured.

Hyphodontiella 39

39. Spores more or less hyaline . (=Chrysoderma) Cerocorticium

40. Basidiome ceraceous, hyphae gelatinized and difficult to discern 40

40. Not as above, hyphae as a rule easily to discern..... Phlebia

41. Basidiome pellicular, basidia in candelabrum-arranged clusters. 41

41. Not as above Athelia

42. Basidia with 2 long sterigmata, spores allantoid 42

42. Differently characterized Cerinomyces

43. Basidiome brown to dark brown, subhymenial hyphae encrusted with resinous matter by age, spores navicular.

.Luellia

43. Not as above .. 44

44. Hymenial surface white, changing to purple in $\mathrm{KOH}$

44. Hymenial surface not changing in $\mathrm{KOH}$ Purpureocorticium

45. Basidia as a rule urniform to suburniform 45

45. Basidia cylindrical to clavate. 46

46. Basidia urniform to cylindrical, often with internal repetition, spores allantoid. 48

46. Not as above Galzinia

47. Basidia on average longer than $30 \mu \mathrm{m}$, spore usually longer than $7 \mu \mathrm{m}$.

47. Basidia on average shorter than $30 \mu \mathrm{m}$, spore usually up to $7 \mu \mathrm{m}$ long. 47

48. Basidia clearly stalked Xylodon

48. Basidia not evidently stalked.... 49

49. Basidia up to $20 \mu \mathrm{m}$ long, tetrasporic 52

49. Basidia more than $20 \mu \mathrm{m}$ long, with a variable number of sterigmata (2-3-4)

50. Basidia 50-75 $\mu \mathrm{m}$ long, spores globose to subglobose, 9-13 $\mu \mathrm{m}$ in diameter Athelopsis

50. Basidia up $30 \mu \mathrm{m}$ long, spores ellipsoid to cylindrical, up to $8 \mu \mathrm{m}$ wide.

51. Basidia bisporic, 20-25 $\mu \mathrm{m}$ long, spores wider than $5 \mu \mathrm{m}$.

51. Basidia with 2-3-4 sterigmata, 27-30 $\mu \mathrm{m}$ long, spores up to $4 \mu \mathrm{m}$ wide

52. Basal hyphae cinnamon encrusted, violaceous in $\mathrm{KOH}$. 50

52. Basal hyphae otherwise

53. Basidia distinctly very thick-walled

53. Not as above Globulicium

54. Basidia small, on average $<20 \mu \mathrm{m}$ long. Globulicium

54. Basidia on average $>20 \mu \mathrm{m}$ long....

55. Spores on average more than $2.5 \mu$ m wide

55. Spores on average less than $2.5 \mu \mathrm{m}$ wide. Athelicium Thujacorticium Ceraceomyces

56. Spores up to $4.5 \mu \mathrm{m}$ wide, thin-walled, on bark of living trees. 53

56. Spores on average more than $5.5 \mu$ m wide, slightly thick-walled, on dead wood

57. Spores cylindrical to ellipsoid, more than $15 \mu \mathrm{m}$ long.

57. Spores usually ellipsoid, up to $15 \mu \mathrm{m}$ long.

Tubulicrinopsis 


\section{Descriptions and comments on genera of corticioid fungi and relatives}

Acanthobasidium Oberw., Sydowia 19(1-6): 45, 1965.

Type species - Aleurodiscus delicatus Wakef., Trans. Br. mycol. Soc. 35(1): 44, 1952.

Basidiome resupinate, effused, thin, hymenophore smooth. Hyphal system monomitic, generative hyphae with clamps. Gloeocystidia and acanthocystidia present. Basidia (pleurobasidia) with some lateral protuberances (acanthobasidia). Basidiospores ornamented, amyloid.

Remarks - Acanthobasidium is characterized within the Aleurodiscus s.l. above all by the pleurobasidia with small digitiform protuberances. As the other genera segregated from Aleurodiscus s.l., it belongs to the Stereaceae in the Russulales (Larsson 2007b).

Acanthocorticium Baltazar, Gorjón \& Rajchenb., Botany 93: 456, 2015.

Type species - Acanthocorticium brueggemannii Baltazar, Gorjón \& Rajchenb. Botany 93: 458, 2015.

Basidiome corticioid, resupinate, adnate, cartilaginous hard when dry, hymenophore smooth to tuberculate, even to rimose. Hyphal system monomitic, generative hyphae simple-septate. Halocystidia abundant in the subiculum and the hymenium, hyaline, finely echinulate at the apex, IKI- to slightly dextrinoid, with a resinous cap. Acanthophyses dominating in the hymenium, cylindrical to clavate, hyaline, with short protuberances in the apical part, dextrinoid. Basidiospores globose, hyaline, smooth and thin-walled, IKI-.

Remarks - Acanthocorticium is characterized by the resupinate, cartilaginous basidiome with smooth to tuberculate hymenophore, and microscopically by the presence of abundant, dextrinoid acanthophyses, apically echinulate halocystidia with a resinous cap, and globose, hyaline, IKIbasidiospores. For further information see Baltazar et al. (2015).

Acanthofungus Sheng H. Wu, Boidin \& C.Y. Chien, Mycotaxon 76: 154, 2000.

Type species - Acanthofungus rimosus Sheng H. Wu, Boidin \& C.Y. Chien, Mycotaxon 76: 155, 2000.

Basidiome pulvinate, hymenial surface smooth. Hyphal system monomitic, hyphae with clamps. Acanthophyses numerous. Gloeocystidia SA+. Basidia clavate, with 4-sterigmata, and a basal clamp. Basidiospores ellipsoid, smooth, thin-walled, amyloid, binucleate. Causing a white pocket rot. Nuclear behaviour heterocytic.

Remarks - Acanthofungus is characterized by the pulvinate basidiome causing a white pocket rot, hyphae clamped, presence of acanthophyses, SA+ gloeocystidia, and smooth amyloid basidiospores. It reminds superficially Xylobolus but differs in the clamped hyphae and heterocytic nuclear behaviour (holocoenocytic and clampless hyphae in Xylobolus). Acanthofungus differs from Acanthophysellum in the white pocket rot and phenoloxidase negative reaction, however micromorphologically they are similar (Wu et al. 2000).

Acanthophysellum Parmasto, Eesti NSV Tead. Akad. Toim., Biol. seer 16: 377, 1967.

Type species - Corticium lividocoeruleum P. Karst., not. Sällsk. Fauna et Fl. Fenn. Förh. 9: 370, 1868.

Basidiome resupinate, thin, adnate. Hyphal system monomitic, generative hyphae with clamps or with simple-septa. Gloeocystidia and acanthocystidia present. Basidia clavate to cylindrical, 2-4-sterigmata. Basidiospores smooth, thin-walled, cylindrical or ellipsoid to allantoid, amyloid.

Remarks - Acanthophysellum was introduced for resupinate species with acanthohyphidia and smooth non-amyloid basidiospores with Corticium lividocoeruleum as the generic type species (Parmasto 1967). The statement about non-amyloid spores must be regarded as a typographical error because later Parmasto (1968) included Acanthophysellum in the tribe Aleurodisceae defined by the amyloid spores. Corticium lividocoeruleum and Thelephora frustulata Pers., the generic type of Xylobolus P. Karst, are closely related phylogenetically and morphologically (Larsson \& Larsson 
2003), and this connection could suggest that Acanthophysellum should be regarded as a synonym of Xylobolus. However, Acanthophysellum differs from Xylobolus in some morphological features and in having white rot (white pocket rot in Xylobolus), phenoloxidase positive reaction and nuclear behaviour astatocoenocytic (holocoenocytic in Xylobolus). Wu et al. (2000) and Boidin \& Gilles (2001) treated Acanthophysium as a synonym of Xylobolus but conserved Acanthophysellum.

Acanthophysium (Pilát) G. Cunn., Bull. N.Z. Dept. Sci. Industr. Res., Pl. Dis. Div. 145: 150, 1963. Basionym - Aleurodiscus subgen. Acanthophysium Pilát, Annls mycol. 24(3/4): 207, 1926.

Type species - Aleurodiscus apricans Bourdot, Rev. Sci. du Bourb. 23(1): 7, 1910.

Remarks - A synonym of Xylobolus P. Karst. See Acanthophysellum and Xylobolus for further comments.

Acia P. Karst., Medd. Soc. Fauna Fl. Fenn. 5: 42, 1879.

Type species - Hydnum fuscoatrum Fr., Novit. fl. svec. 2: 39, 1814

Remarks - A homonym of Acia Schreb. 1791 (Rosaceae) and a synonym of Phlebia Fr.

Aciella (P. Karst.) P. Karst., Finlands Basidsvampar 11: 148, 1899.

Type species - Phaeodon tomentosus Schrad. ex J. Schröt., Kryptogamen-Flora von Schlesien, 3-1(4): 458, 1888.

Remarks - A homonym of Aciella Tieghem 1894 (Loranthaceae) and a synonym of Tomentella Pers. ex Pat.

Acladium Link, Mag. Gesell. naturf. Freunde, Berlin 3(1-2): 11, 1809. 1809.

Type species - Acladium conspersum Link, Mag. Gesell. naturf. Freunde, Berlin 3(1-2): 11,

Remarks - A name based on an anamorph. Anamorphic state of Botryobasidium Donk.

Actinostroma Klotzsch, Nova Acta Phys.-Med. Acad. Caes. Leop.-Carol. Nat. Cur., Suppl. 1 19: 236, 1843.

Type species - Actinostroma infunfibuliforme Klotzsch, Nova Acta Phys.-Med. Acad. Caes. Leop.-Carol. Nat. Cur., Suppl. 1 19: 237, 1843.

Remarks - A synonym of Cymatoderma Jungh.

Adustomyces Jülich, Persoonia 10(3): 325, 1979.

Type species - Stereum repandum var. lusitanicum, Broteria (Bot.) 11: 76, 1913.

Remarks - A synonym of Radulomyces M.P. Christ. Adustomyces is close or identical to Radulomyces, but it differs in its crustaceous thick basidiome, brown hyphae and a blackening trama in $\mathrm{KOH}$.

Adustoporia Audet, Mushrooms nomenclatural novelties 11: 1, 2017.

Type species - Polyporus sinuosus Fr., Syst. mycol. (Lundae) 1: 381, 1821.

Remarks - According to the original description by Audet (2017j), Adustoporia differs from Antrodia s.str. by only resupinate basidiomes, pale brown pore surface when fresh, lack of false skeletocystidia, smaller basidia, smaller suballantoid basidiospores with no tapering distal end and from Amyloporia s.str. by no yellow basidiome, different hyphal system, inamyloid skeletal hyphae, and by the not similar cultural characteristics. According to Audet (2017j), Adustoporia is supported by the molecular analyses in Ortiz-Santana et al. (2013), Spirin et al. (2015) and Binder et al. (2013).

Aegerita Pers., Neues Mag. Bot. 1: 120, 1794.

Type species - Aegerita candida Pers., Neues Mag. Bot. 1: 120, 1794. 
Remarks - A name based on an anamorph. Aegerita is the anamorphic state of Bulbillomyces Jülich.

Aegeritina Jülich, Int. J. Mycol. Lichenol. 1(3): 282, 1984.

Type species - Aegerita tortuosa Bourdot \& Galzin, Hyménomyc. de France (Sceaux): 298, 1928.

Remarks - A name based on an anamorph. Aegeritina is the anamorphic genus designated for Subulicystidium Parmasto. It consists in small globules composed of tortuous to somewhat inflated clamped hyphae with the subulate and characteristically encrusted cystidia of the teleomorph state. It differs from Aegerita, the anamorph of Bulbillomyces farinosus (Bres.) Jülich by the absence of cystidial elements in the latter.

Aegis Gómez-Mont., Rajchenb. \& Robledo, Mycosphere 8 (6): 1265, 2017. 2017.

Type species - Aegis boa Gómez-Mont., Rajchenb. \& Robledo, Mycosphere 8 (6): 1265,

Basidiome annual, resupinate, effused-reflexed to pileate, pileal surface glabrous to tomentose, usually white to light ivory, azonate, pores round, 5-6 per mm, context thin, fibrous, white. Hyphal system monomitic with thin- to thick-walled clamped generative hyphae, metachromatic, IKI-. Basidia clavate, with four sterigmata. Basidiospores smooth, allantoid, hyaline, thin-walled, IKI-.

Remarks - Aegis is characterized microscopically by a monomitic hyphal system with clamp connections and particular thick-walled hyphae that present inflated and widened portions and constrictions, and allantoid basidiospores. The phylogenetic analysis by Gómez-Montoya et al. (2017) recovered the general topology of previous works, with Grifola, Antrodiella aurantilaeta (Corner) T. Hatt. \& Ryvarden, and Aegis presenting an isolated position, called 'Grifola' clade.

Aldridgea Massee, Grevillea 20(no. 96): 121, 1892.

Type species - Aldridgea gelatinosa Massee, Grevillea 20(no. 96): 121, 1892 (=Thelephora puteana Schumach., Enum. pl. (Kjbenhavn) 2: 397, 1803).

Remarks - A synonym of Coniophora DC.

Aleurobotrys Boidin, Bull. trimest. Soc. mycol. Fr. 101(4): 340, 1985.

Type species - Aleurodiscus botryosus Burt., Ann. Mo. bot. Gdn 5: 198, 1918.

Basidiome resupinate, thin, hymenophore smooth. Hyphal system monomitic, generative hyphae with simple septa. Gloeocystidia present. Acanthophyses with short amyloid branches (botryophyses). Basidiospores ornamented, amyloid.

Remarks - Aleurobotrys is a monotypic genus segregated from Aleurodiscus for species with sulfocystidia, ornamented basidiospores and amyloid vegetative structures called botryophyses (acanthophyses with short and obtuse dichotomous branches). The phylogenetic analysis by Larsson \& Larsson (2003), confirm Aleurobotrys botryosus as distinct from Aleurodiscus, and it may be related to Gloeomyces but no further conclusions about relationships can be drawn. Wu et al. (2001) found that A. botryosus is close to Aleurodiscus cerussatus, and they concluded that the phylogenetic importance of the amyloid reaction of the botryophyses is doubtful (Bernicchia \& Gorjón 2010).

Aleurocorticium P.A. Lemke, Can. J. Bot. 42: 724, 1964.

Type species - Corticium acerinum Pers., Observ. mycol. (Lipsiae) 1: 37, 1796.

Remarks - A synonym of Dendrothele Höhn. \& Litsch.

Aleurocystidiellum P.A. Lemke, Can. J. Bot. 42: 277, 1964.

Type species - Stereum subcruentatum Berk. \& M.A. Curtis, Proc. Amer. Acad. Arts \& Sci. 4: 123, 1858. 
Basidiome annual to perennial, cupulate to substereoid, margin determinate, subcoriaceous, hymenophore smooth, abhymenial sterile surface glabrous. Hyphal system monomitic or dimitic, generative hyphae with clamps. Basidia subclavate, with 4-sterigmata, and a basal clamp. Basidiospores ovoid to broadly ellipsoid, apiculate, thick-walled, minutely verruculose in Melzer's, amyloid.

Remarks - Aleurocystidiellum was created by Lemke (1964a) to include dimitic species with discoid basidiome and large, minutely verrucose and amyloid spores with Aleurocystidiellum subcruentatum (Berk. \& M.A. Curtis) P.A. Lemke as type species. Aleurocystidiellum subcruentatum was previously placed in Aleurodiscus because of the discoid basidiome and the amyloid basidiospores. However, the skeletal hyphae ending into the hymenium as cystidia-like, more or less encrusted elements made Aleurocystidiellum subcruentatum a very distinct species from Aleurodiscus amorphus (Pers.) J. Schröt., the type of Aleurodiscus. Aleurodiscus disciformis (DC.) Pat. was investigated by Boidin et al. (1968) and it showed almost identical cultural characteristics but having monomitic hyphal system and SA+ gloeocystidia. Boidin et al. (1968) intended to make the appropriate combination in Aleurocystidiellum but failed to include a reference to the basionym. Later, Telleria (1990) provided a correct combination as Aleurocystidiellum disciforme (DC.) Telleria. Hallenberg \& Parmasto (1998) found that morphological and molecular data supported a close relationship between the two species, also confirmed by molecular studies by Wu et al. (2001), Larsson \& Larsson (2003) and Binder et al. (2005). Basidiospores in SEM showed identical wart-like ornamentation in Aleurocystidiellum subcruentatum and Aleurocystidiellum disciforme, and that is clearly different from the rod-like protuberances present in Aleurodiscus amorphus that gives an aculeate appearance to the spores (Hallenberg \& Parmasto 1998). The skeletal cystidia in Aleurocystidiellum subcruentatum and the gloeocystidia in Aleurocystidiellum disciforme may be homologous, both representing modifications of the gloeoplerous hyphae that characterize other taxa in the russuloid clade (Hibbett \& Thorn 2001, Wu et al. 2001), however subicular hyphae in A. disciforme have thickened walls with clamps difficult to discern and with a skeletoid aspect. Nevertheless, the two species have a similar ecology, occurring both on barks of living trees (Bernicchia \& Gorjón 2010).

Aleurocystis Lloyd ex G. Cunn., Trans. \& Proc. Roy. Soc. New Zealand 84: 234, 1956.

Type species - Aleurodiscus capensis Lloyd, Lloyd Mycol. Notes 62: 930, 1920. (=Corticium hakgallae Berk. \& Broome, J. Linn. Soc. 14: 72, 1873).

Basidiome cupulate to resupinate, pale yellow to ochre, annual, gelatinous and tough when fresh, horny and dense when dry, hymenial surface smooth to tuberculate. Hyphal system monomitic, generative hyphae with clamps, thick-walled in the subiculum and straight to branched, gelatinized in $\mathrm{KOH}$. Paraphysoid hyphae to dendrohyphidia present (in the type) or absent. Cystidia as metuloids or encrusted hyphal ends, thick-walled, projecting or enclosed. Gloeocystidia present in one species. Basidia clavate, with 4-sterigmata, and a basal clamp. Basidiospores large, ellipsoid, smooth, thin- to thick-walled, non-amyloid.

Remarks - Aleurocystis reminds macromorphologically Aleurodiscus s.l., by the cupulate basidiome, but it differs in the encrusted cystidial elements, and inamyloid smooth basidiospores. Dendrohyphidia are present in the generic type distributed in South Africa and in Aleurocystis magnispora (Burt.) P.A. Lemke from Jamaica, Colombia, and Southern Brazil, but this structure lacks in A. gloeocystidiata Rajchenb. \& Robledo, from Argentina, that also differs in possessing SA-gloeocystidia.

Aleurodiscus Rabenh. ex J. Schröt, in Cohn, Krypt.-Fl. Schlesien (Breslau) 3(1): 429, 1888.

Type species - Peziza amorpha Pers., Syn. meth. fung. 2: 657, 1801.

Basidiome resupinate or discoid, margin variable, in some species more or less reflexed or undifferentiated. Hyphal system monomitic or dimitic, hyphae clamped or simple-septate. A variable set of sterile elements may be present (gloeocystidia, acanthophyses, dendrophyses, paraphysoid hyphae, encrusted cystidia, encrusted or smooth skeletocystidia). Basidia medium to 
large, with 4-sterigmata, simple-septate or with a basal clamp. Basidiospores smooth or ornamented, amyloid.

Remarks - Aleurodiscus in a very broad sense, includes species with several sterile elements (acanthophyses, dendrophyses, paraphysoid hyphae, ett.), hyphae clamped or simple-septate, gloeocystidia absent or present and, amyloid basidiospores either smooth or ornamented. In a restricted sense, Aleurodiscus comprises those species with amyloid basidiospores but no acanthophyses, botryophyses, acanthobasidia, or encrusted sterile elements. Even a sensu lato Aleurodiscus concept is useful to quickly identify a group of species, morphological as well molecular evidence to seem indicate that Aleurodiscus can be split into more homogeneous groups. However, we agree that further molecular studies with more species and specimens, included those from tropics and Southern Hemisphere is needed to clarify the taxonomic situation of Aleurodiscus s.l. For a detailed information and discussion see also Lemke (1964a,b), Oberwinkler (1965), Parmasto (1967), Boidin et al. (1985), Telleria \& Melo (1995), Wu et al. (2000), Wu et al. (2001), Larsson \& Larsson (2003), Larsson et al. (2004), Gorjón et al. (2013), Dai \& He (2016), Tian et al. (2018).

Aleuromyces Boidin \& Gilles, Bull. Soc. Mycol. Fr. 117(3): 176, 2001.

Type species - Aleurodiscus gabonicus Boidin, Lanq. \& Gilles, in Boidin et al. Bull. Soc. Mycol. Fr. 101(4): 352, 1986.

Basidiome resupinate, effused, adnate, whitish, hymenial surface smooth. Hyphal system monomitic, hyphae with simple-septa. Acanthophyses present, inamyloid and indextrinoid. Gloeocystidia often with a moniliform apex, thin to slightly thick-walled, SA-. Basidia ovoid, with 4-sterigmata, with a simple basal septum. Basidiospores ovoid, verrucose, amyloid, spore print white.

Remarks - Aleuromyces is characterized by the amyloid and ornamented basidiospores, presence of acanthophyses and simple-septate hyphae. It was proposed to accommodate Aleurodiscus gabonicus, but the relationships with other genera within Aleurodiscus s.str. is unclear. Núñez \& Ryvarden (1997) described clamped hyphae in A. gabonicus contrary to Boidin \& Gilles (2001) that described only verticillate clamps in the culture, not in the basidiome.

Alysidium Kunze, in Kunze \& Schmidt, Mykologische Hefte (Leipzig) 1: 11, 1817. 1817.

Type species - Alysidium fulvum Kunze \& J.C. Schmidt, Mykologische Hefte (Leipzig) 1: 11,

Remarks - A name based on an anamorph. Anamorphic state of Botryobasidium Donk.

Allescheriella Henn., Hedwigia 36(4): 244, 1897.

Type species - Allescheriella uredinioides Henn. [as 'uredinoides'], Hedwigia 36(4): 244, 1897.

Remarks - A name based on an anamorph. Anamorphic state of Botryobasidium Donk.

Alutaceodontia (Parmasto) Hjortstam \& Ryvarden, Syn. Fung. (Oslo) 15: 7, 2002.

Basionym - Hyphodontia sect. Alutaceodontia Parmasto, Consp. System. Corticiac. (Tartu): 124, 1968.

Type species - Hydnum alutaceum Fr., Syst. mycol. (Lundae) 1: 417, 1821.

Basidiome resupinate, effused, hymenophore odontioid with small aculei. Hyphal system monomitic, hyphae with clamps, and with distinct walls. Cystidia with tramal origin (pseudocystidia) thin-walled, cylindrical or with several constrictions to moniliform, apically obtuse and, aseptate. Basidia subclavate to suburniform, with 4-sterigmata and a basal clamp. Basidiospores allantoid, smooth, thin-walled, IKI-, CB-.

Remarks - Alutaceodontia is separated from Hyphodontia s.l. mainly by the thin-walled cystidia and allantoid basidiospores (Hjortstam \& Ryvarden 2002a). It reminds in some respects Chaetoporellus, but the latter is easily separated by the typical poroid hymenophore. It seems to 
belong to the Hymenochaetales and related to Kneiffiella, morphologically separated by the thickwalled tramal cystidia (Larsson et al. 2006, Larsson 2007b). Alutaceodontia and Chaetoporellus are treated as a synonym of Kneiffiella P. Karst. by Riebesehl \& Langer (2017).

Alytosporium Link, in Willdenow, Sp. Pl., Ed. 4 6(1): 23, 1824.

Type species - Sporotrichum badium Link, Mag. Gesell. naturf. Freunde, Berlin 3(1-2): 12, 1809.

Remarks - A synonym of Tomentella Pers. ex Pat.

Amaurodon J. Schröt., in Cohn, Krypt.-Fl. Schlesien (Breslau) 3.1(25-32): 461, 1888.

Type species - Sistotrema viride Alb. \& Schwein., Consp. fung. (Leipzig): 262, 1805.

Synonyms - Hypochnopsis P. Karst., Lazulinospora Burds. \& M.J. Larsen, Tomentellago Hjortstam \& Rvarden.

Basidiome resupinate, detachable, mostly arachnoid or pelliculose, continuous, hymenophore bluish when fresh, yellow-green when dry, smooth, hydnoid or poroid, concolorous with subiculum, sterile margin usually indeterminate or byssoid and paler than hymenophore. Hyphal system monomitic, hyphal cords absent or rarely present only in subiculum, subicular and subhymenial hyphae simple-septate or clamped, thin-walled, hyaline or sometimes partly bluish violet in $\mathrm{KOH}$. Cystidia absent. Basidia utriform, not stalked, often sinuous, hyaline or partly violet in $\mathrm{KOH}$, hyaline in distilled water, with 4-sterigmata. Basidiospores slightly globose or ellipsoid, smooth, verrucose and bi- or trifurcate, mostly violet in $\mathrm{KOH}$, hyaline or yellowish in distilled water.

Remarks - Amaurodon is characterized by the bluish basidiomes that become yellowish green when dry and basidiospores, smooth or ornamented, bluish to violet in $\mathrm{KOH}$.

Amaurohydnum Jülich, Persoonia 9(4): 455, 1978.

Type species - Amaurohydnum flavidum Jülich, Persoonia 9(4): 455, 1978.

Basidiome annual, resupinate, effused, adnate, membranaceous, hymenial surface minutely hydnoid, pale coloured. Hyphal system monomitic, hyphae hyaline, cylindrical to torulose, distinct to indistinct, with clamps. Cystidia absent. Basidia distinctly clavate, small, 4-spored, with a basal clamp. Basidiospores hyaline, thin-walled, smooth, small, inamyloid.

Remarks - Amaurohydnum is characterized by the inconspicuous hydnoid basidiomes, absence of cystidial elements, and ellipsoid, smooth and inamyloid basidiospores (Jülich 1978a). The phylogenetic relationships of Amaurohydnum are unclear because there are no available sequences (Larsson 2007b). Monotypic genus.

Amauromyces Jülich, Persoonia 9(4): 456, 1978.

Type species - Amauromyces pallidus Jülich, Persoonia 9(4): 456, 1978.

Basidiome resupinate, effused, adnate, thin, crust-like, ceraceous, hymenial surface pale coloured. Hyphal system monomitic, hyphae hyaline, thin-walled in subhymenium, distinctly thickwalled and very wide in the trama, with clamps at all primary septa; wall of basal hyphae swelling and partly dissolving in $\mathrm{KOH}$. Cystidia hyaline, thick-walled, smooth or encrusted as lamprocystidia, often two-celled with clamped septa. Basidia hyaline, clavate to flexuouscylindrical, 4-spored, with a basal clamp. Basidiospores hyaline, thin-walled, smooth, ellipsoid, small, IKI-, CB-.

Remarks - Amauromyces is characterized by the thick-walled hyphae and cystidia, the hyphae swelling and dissolving in KOH (Jülich 1978a).

Ambivina B. Katz, Nova Hedwigia 25: 811, 1974.

Type species - Ambivina filobasidia B. Katz, Nova Hedwigia 25: 811, 1974.

Remarks - Based on invalidly published species. See Katz (1974). 
Amethicium Hjortstam, Mycotaxon 17: 557, 1983.

Type species - Amethicium rimosum Hjortstam, Mycotaxon 17: 557, 1983.

Basidiome resupinate, effuse, adnate, hymenial surface smooth, moderately soft, in the type species bluish to purplish. Hyphal system dimitic, basal hyphae intricately interwoven, binding hyphae thin- to moderately thick-walled, frequently branched, non-dextrinoid, subhymenial hyphae densely arranged, short-celled, thin-walled, with clamps. Cystidia absent. Basidia terminal, clavate, with 4-sterigmata, and a basal clamp. Basidiospores ellipsoid, smooth, thin-walled, hyaline, IKI-, CB-.

Remarks - Amethicium is characterized by the basal binding hyphae, absence of cystidia, and smooth, negative in Melzer's reagent, and acyanophilous basidiospores (Hjortstam 1983). It reminds Cericium that also possess binding hyphae, but the latter has distinct cystidia and lacks the violet colours of Amethicium.

Amphinema P. Karst., Bidr. Känn. Finl. Nat. Folk 51:228, 1892.

Type species - Thelephora byssoides Pers., Syn. meth. Fung. 2: 577, 1801.

Synonyms - Sphaerophora Bonord, Diplonema P. Karst.

Basidiome resupinate, pellicular, rarely membranaceous, hymenophore smooth or finely velutine due to the projecting cystidia, margin usually with rhizomorphs. Hyphal system monomitic, hyphae yellowish, more or less thin-walled, loosely interwoven, with clamps at all septa (with simple-septa in Amphinema angustispora), usually encrusted. Cystidia hyphoid (leptocystidia), cylindrical, projecting, with clamped septa, slightly thick-walled, yellowish, covered with small granules. Basidia clavate, with 4-sterigmata, and a basal clamp. Basidiospores ellipsoid (fusiform in Amphinema angustispora), smooth, slightly thick-walled, hyaline, inamyloid, indextrinoid, stained in cotton blue but not truly cyanophilous.

Remarks - Although the relationship with other corticioid genera seems unclear, Eriksson \& Ryvarden (1973) indicated some similarities with Hyphodontia in the hyphal characters and the shape of the clamps and basidia. Rattan (1977) pointed a relation with Leucogyrophana due to the reaction of the spore wall with cotton blue. Molecular studies by Larsson et al. (2004) showed the relation between Amphinema byssoides and Athelia, Athelopsis, Byssorticium, Tylospora, and Piloderma in the athelioid clade, also confirmed by Larsson (2007b) including Amphinema in the family Atheliaceae Jülich. The type species of Amphinema is mycorrhizal and its basidiome occurs on several substrata.

Amyloathelia Hjortstam \& Ryvarden, Mycotaxon 10(1): 201, 1979.

Type species - Corticium amylaceum Bourdot \& Galzin, Bull. Soc. mycol. Fr. 27(2): 259, 1911.

Basidiome resupinate, adnate, membranaceous to pellicular; hymenophore smooth, whitish to ochraceous, margin fibrillose to indeterminate. Hyphal system monomitic; hyphae clamped, basal hyphae straight, subhymenial irregular to sinuous. Cystidia absent but paraphysoid hyphal ends may be present. Basidia clavate, 4-sterigmata, and with a basal clamp. Basidiospores ellipsoid to subglobose, smooth to ornamented, thick-walled, with a strong bluish-violet reaction in Melzer's reagent.

Remarks - Amyloathelia differs from other corticioid species with amyloid basidiospores in the absence of sterile elements like cystidia, gloeocystidia, dendrohyphae or acantohyphae (only in A. amylacea some paraphysoid hyphae are present). The strongly amyloid spore-wall separates Amyloathelia from Dendrothele that presents dendrohyphae more or less numerous, and commonly grows on the bark of living trees (Hjortstam \& Ryvarden 1979b). Aleurodiscus s.l. also presents several sterile elements. Amyloathelia is classified in Atheliales Jülich by Boidin et al. (1998), however, molecular studies by Boidin et al. l.c., Binder et al. (2005) and Larsson (2007b) does not clarify the relationships with other corticioid genera. 
Amylobasidium Ginns, Mycologia 80(1): 63, 1988.

Type species - Amylobasidium tsugae Ginns, Mycologia 80(1): 63, 1988.

Basidiome pulvinate, sometimes confluent, margin abrupt, determinate. Hymenial surface smooth, cream coloured. Hyphal system monomitic, hyphae thin- to thick-walled, with clamps, IKI-, acyanophilous. Dendrophyses present, forming a catahymenium. Cystidia absent. Basidia utriform to cylindrical, elongate, irregularly thickened over the lower part, with 4-sterigmate, amyloid, especially where thickened. Basidiospores broadly ellipsoid, large, smooth, thin-walled, hyaline, IKI-, CB-.

Remarks - It is characterized by the amyloid basidial walls, presence of dendrohyphae, pulvinate basidiomes and large basidiospores wider than $12 \mu \mathrm{m}$ (Ginns 1988). Morphologically it seems to be related with Corticium but differing in the amyloid basidia.

Amylocorticiellum Spirin \& Zmitr., Mikol. Fitopatol. 36(1): 22, 2002.

Type species - Corticium subillaqueatum Litsch., Annls mycol. 39(2/3): 128, 1941.

Basidiome resupinate, effused, pellicular to membranaceous, margin fibrillose. Hymenophore smooth to merulioid, pale coloured. Hyphal system monomitic, hyphae with evident clamps. Cystidia (leptocystidia) present or absent. Basidia clavate, sinuous, with a basal clamp. Basidiospores ellipsoid, smooth, thick-walled, amyloid, cyanophilous.

Remarks - Zmitrovich \& Spirin (2002) described the genus Amylocorticiellum with Corticium subillaqueatum as the generic type species and included Corticium cremeoisabellinum Litsch., Thelephora mollis Fr. and Amylocorticiellum sinuosus Spirin \& Zmitr. These species with distinct amyloid spore wall (greyish in Melzer's reagent) were previously included in Hypochniciellum when Hjortstam (1981) emended the generic circumscription. Hypochniciellum originally included only Leptosporomyces ovoideus Jülich characterized by thick-walled and cyanophilous basidiospores and no reaction in Melzer. Molecular studies include Corticium subillaqueatum the generic type of Amylocorticiellum in the Amylocorticiaceae Jülich, but Leptosporomyces ovoideus the type species of Hypochniciellum, is still not molecularly analysed (Larsson 2007b). Gorjón et al. (2011) added three species to Amylocorticellum considering Hypochniciellum restricted to the generic type.

Amylocorticium Pouzar, Česká Mykol. 13(1): 11, 1959. 1881.

Type species - Corticium subsulphureum P. Karst., Meddn Soc. Fauna Flora fenn. 6: 12,

Basidiome resupinate, at first slightly pellicular, after thicker and membranaceous; hymenophore smooth, usually white to yellowish. Hyphal system monomitic, hyphae with clamps or simple septa, thin-walled, subicular hyphae loosely interwoven, the subhymenial hyphae arranged in a denser tissue. Cystidia absent or present, in any case only hyphoid. Basidia subclavate to clavate, with 4-sterigmata, with simple septa or a basal clamp. Basidiospores narrowly ellipsoid to allantoid, up to $3 \mu \mathrm{m}$ wide, thin-walled, smooth and amyloid.

Remarks - Amylocorticium is above all characterized by the smooth and amyloid basidiospores, no other remarkable elements are present. Amylocorticium shares similar features with Anomoporia (Polyporaceae), except for the poroid hymenophore (Ryvarden \& Gilbertson 1993). Molecular analysis by Larsson et al. (2004) pointed out this relation, indeed Amylocorticium, Anomoporia, Ceraceomyces, and Hypochniciellum share the amyloidity of the basidiospores (except Ceraceomyces) and are recovered in the same subclade.

Amylodontia Nikol., Nov. sist. Niz. Rast. 4: 238, 1967.

Type species - Amylodontia parmastoi Nikol., Nov. sist. Niz. Rast. 4: 238, 1967.

Remarks - A synonym of Dentipellis Donk.

Amylofungus Sheng H. Wu, Mycologia 87(6): 886, 1995.

Type species - Corticium corrosum G. Cunn. in Trans. Roy. Soc. N. Z. 82: 286, 1954. 
Basidiome resupinate, effuse, hymenial surface smooth. Hyphal system monomitic, hyphae simple-septate, amyloid. Gloeocystidia numerous, clavate or cylindrical, SA+, amyloid. Basidia utriform, with 4-sterigmate, simple-septate at the base, amyloid. Basidiospores globose or subglobose, smooth, thin-walled, amyloid.

Remarks - Amylofungus is characterized by the presence of amyloid hyphae, gloeocystidia, basidia, and basidiospores (Wu 1995). According to Larsson (2007b), it seems to be close to Vesiculomyces. Hjortstam (1995) also placed Corticium corrosum in Vesiculomyces.

Amylohyphus Ryvarden, Bull. Jard. Bot. Nat. Belg. 48: 81, 1978.

Type species - Amylohyphus africanus Ryvarden, Bull. Jard. Bot. Nat. Belg. 48: 82, 1978.

Basidiome resupinate, adnate and hard, hymenial surface smooth along the margin, uneven and warted in more mature parts, light brown, ochraceous at the margin. Hyphal system monomitic, generative hyphae with clamps, amyloid. Catahymenium present with numerous thin- to thickwalled amyloid cystidia, partly as elongated thick-walled hyphae, partly as bulbous to subulate cystidia, smooth to encrusted. Basidia clavate, with 4-sterigmate, and a basal clamp. Basidiospores cylindrical, large, thin-walled, inamyloid.

Remarks - Amylohyphus is characterized by the amyloid reaction in hyphae and cystidia. Cystidial elements are very variable, from encrusted hyphoid to bulbous or subulate, all with characteristic thickened and amyloid walls (Ryvarden 1978).

Amylonotus Ryvarden, Norwegian Journal of Botany 22 (1): 26, 1975. 1975.

Type species - Amylonotus africanus Ryvarden, Norwegian Journal of Botany 22 (1): 26,

Basidiome annual, sessile, pileate, effused-reflexed or resupinate, soft coriaceous when fresh, coriaceous to brittle when dry. Pileal surface cinnamon to dark brown, first finely tomentose, becoming smooth with age. Pore surface pale orange, isabelline, pale cinnamon to brown; pores large, labyrinthine to daedaleoid. Context pale cinnamon to brownish orange, membranous to fibrous. Tubes leathery to fibrous when dry. Hyphal system dimitic, generative hyphae with clamp connections, skeletal hyphae dextrinoid. Gloeoplerous hyphae present or absent. Gloeocystidia occasionally present or absent. Cystidia absent. Basidiospores ellipsoid to subglobose, hyaline, thin- to slightly thick-walled, finely asperulate, $\mathrm{IKI}+, \mathrm{CB}+$. Causing a white rot.

Remarks - Amylonotus is easy to recognize in the microscope because of the asperulate amyloid spores and the dimitic hyphal system with clamped generative hyphae (Ryvarden 1975). Externally it may remind of an Inonotus. For a phylogenetic discussion see Chen et al. (2016).

Amyloporia Bondartsev \& Singer ex Singer, Mycologia 36 (1): 67, 1944.

Type species - Polyporus vulgaris var. calceus Fr., Syst. mycol. (Lundae) 1: 381, 1821.

Basidiome annual, resupinate, often widely effused, adnate, soft when fresh, crumbly and chalky when dry. Pore surface white, to sulphurous yellow or cream when fresh, fading on drying and storing to almost pure white or pale cream, pores circular. Hyphal system dimitic, generative hyphae with clamps, thin-walled, hyaline, skeletal hyphae predominant, semisolid, straight to slightly sinuous, non-septate, unbranched to occasionally dichotomously branched, weakly amyloid but reaction variable and most easily seen in hyphal masses and in fresh condition. Cystidia none, but fusoid, inconspicuous, non-projecting cystidioles occur scattered among the basidia. Basidia clavate, 4-sterigmate, with a basal clamp. Basidiospores allantoid to cylindrical or ellipsoid, hyaline, smooth, thin-walled, negative in Melzer's reagent. Causing a brown cubical rot.

Remarks - Amyloporia is similar to Antrodia in the hyphal system, cylindrical to ellipsoid basidiospores non-reacting in Melzer's reagent, and in the type of rot. It is separated by the amyloidity of the vegetative hyphae; phylogenetical studies confirm this separation (Rajchenberg et al. 2011). 
Amylosporia B.K. Cui, C.L. Zhao \& Y.C. Dai, Fungal Diversity 97: 164, 2019.

Type species - Perenniporia hattorii Y.C. Dai \& B.K. Cui, Ann. Bot. Fenn. 48(3): 224, 2011.

Basidiome annual, resupinate to effused-reflexed, adnate, soft corky to corky when fresh, becoming corky to fragile upon drying. Pore surface cream to buff when fresh, becoming cinnamon-buff when bruised, pale yellowish brown upon drying. Subiculum cream to buff, corky. Tubes concolorous with the pore surface, corky to fragile. Hyphal system dimitic; generative hyphae bearing clamp connections; skeletal hyphae weakly amyloid, CB+. Cystidia absent; cystidioles present. Basidiospores ellipsoid, truncate, hyaline, thick-walled, smooth, weakly amyloid, $\mathrm{CB}+$.

Remarks - Amylosporia hattorii (Y.C. Dai \& B.K. Cui) B.K. Cui, C.L. Zhao \& Y.C. Dai, was firstly described in Perenniporia Murrill from tropical China (Dai et al. 2011). It is characterized by an annual growth habit, amyloid, and cyanophilous skeletal hyphae, and ellipsoid, truncate and amyloid basidiospores. Amylosporia differs from Perenniporia by its both amyloid skeletal hyphae and amyloid basidiospores (Cui et al. 2019).

Amylosporomyces S.S. Rattan, Biblthca Mycol. 60: 244, 1977.

Type species - Amylosporomyces echinosporum S.S. Rattan, Biblthca Mycol. 60: 245, 1977.

Remarks - A synonym of Gloeodontia Boidin. Larsson \& Larsson (2003) studied the type material of A. echinosporum and found it similar to Gloeodontia subasperispora (Litsch.) E. Larss. \& K.H. Larss.

Amylosporus Ryvarden, Norwegian Journal of Botany 20 (1): 1, 1973.

Type species - Polyporus campbellii Berk., Hooker’s J. Bot. Kew Gard. Misc. 6: 228, 1854.

Basidiome terrestrial or lignicolous, stipitate to sessile. Pileus ochraceous to buff. Context white to pale brown. Pores small and entire. Hyphal system dimitic. Generative hyphae hyaline thin-walled both with simple septa and multiple verticillate clamps, often on the same hyphae. Skeletal hyphae hyaline, thick-walled to almost solid, unbranched or moderately branched. Gloeoplerous hyphae present or absent. Gloeocystidia absent. Cystidia absent. Basidiospores broadly ellipsoid to subglobose, thin- to slightly thick-walled, finely asperulate, $\mathrm{IKI}+, \mathrm{CB}+$ or $\mathrm{CB}-$. Causing white rot.

Remarks - Amylosporus is a rather distinct genus with its sessile to stipitate basidiomes and a dimitic hyphal system with two types of septation on the generative hyphae, a rather rare characteristic in the Polyporaceae. Gloeoplerous hyphae are also quite rare among the stipitate genera. The basidiospores are finely warted and not like those of Bondarzewia which as coarse reticulate ornamentation. For a recent phylogeny see Chen \& Shen (2014) and Chen et al. (2016).

Amylostereum Boidin, Revue Mycol., Paris 23(3): 345, 1958.

Type species - Thelephora chailletii Pers., Mycol. eur. (Erlanga) 1: 125, 1822.

Basidiome resupinate, effuse-reflexed or pileate, hymenophore smooth, grey to brownish coloured. Hyphal system monomitic to dimitic, generative hyphae with clamps, thin-walled, hyaline, skeletal hyphae thick-walled and brown. Cystidia (lamprocystidia) fusiform, encrusted in the apical part, thick-walled, yellowish brown. Basidia subclavate, thin-walled, with 4-sterigmata, and basal clamp. Basidiospores cylindrical to narrowly ellipsoid, smooth, thin-walled, amyloid.

Remarks - Amylostereum is mainly characterized by the lamprocystidia and the amyloid smooth basidiospores (Boidin \& Lanquetin 1984). According to molecular data by Binder et al. (2005), Amylostereum appears closely related to Echinodontium in the russuloid clade. In the molecular study by Larsson (2007b), Amylostereum and Echinodontium are recovered in separate subclades of the russuloid clade. Amylostereum included symbiotic fungi of wood wasps (Sirex spp., Urocerus spp.) and most dispersal happens via asexual spores by its winged symbionts, and when they act as invasive species can cause ecological and economic importance damages (Nielsen et al. 2009). 
Amyloxenasma (Oberw.) Hjortstam \& Ryvarden, Syn. Fung. (Oslo) 20: 34, 2005.

Basionym - Xenasmatella subg. Amyloxenasma Oberw., Sydowia, Ann. Mycol. Ser. II, 19: 35, 1965.

Type species - Corticium grisellum Bourdot, Rev. Sci. Bourb. Centr. Fr. 35:17, 1922.

Basidiome resupinate effused, thin to moderately thick, hard when dry, smooth to more or less tuberculate, greyish often with a bluish tint. Hyphal system monomitic, hyphae with clamps, more or less gelatinized and often with irregular ampullate septa. Cystidia absent. Basidia usually short, pleural, with 4-sterigmata, and a basal clamp. Basidiospores allantoid to reniform, smooth, thin-walled and amyloid.

Remarks - Amyloxenasma was segregated from Phlebiella as independent genus by Hjortstam \& Ryvarden (2005) ranking to generic level the subgenus Amyloxenasma Oberw. It includes species with pleurobasidia and smooth amyloid basidiospores. The phylogenetic position of Amyloxenasma is unclear, but it could belong to the Amylocorticiaceae because the amyloid spore wall is indicative of this group, but it differs from other genera included in this family in the morphology of the basidia, typically pleurobasidiate (Larsson 2007b).

Anomoloma Niemelä \& K.H. Larss., Mycotaxon 100: 312, 2007.

Type species - Polyporus albolutescens Romell, Arkiv Botanik 11: 11, 1911.

Basidiome annual, resupinate, loosely attached, soft to brittle, margin with rhizomorphs, hymenial surface poroid, pores round to angular, whitish, yellow or pinkish. Hyphal system monomitic, generative hyphae with clamps, thin- to thick-walled, encrusted or smooth. Cystidia absent. Basidia clavate, with 4-sterigmata, and a basal clamp. Basidiospores usually ellipsoid to subglobose, smooth, thin-walled, amyloid. On dead wood of conifers and hardwood, causing a white rot.

Remarks - Anomoloma is closely related to Anomoporia differing in species with margin rhizomophic and causing a white rot (Niemelä \& Larsson 2007). Phylogenetic relationship has been studied by Song et al. (2016).

Anomoporia Pouzar, Ceska Mykol. 20: 172, 1966.

Type species - Polyporus bombycinus Fr., Elench. Fung. 1: 117, 1828.

Basidiome annual, resupinate, loosely attached, soft to brittle, margin without rhizomorphs, hymenial surface poroid, pores round to angular, whitish, yellow or pinkish. Hyphal system monomitic, generative hyphae with clamps, thin- to thick-walled, encrusted or smooth. Cystidia absent. Basidia clavate, with 4-sterigmata, and a basal clamp. Basidiospores usually ellipsoid to subglobose, smooth, thin-walled, amyloid. On dead wood of conifers and hardwood, causing a brown rot.

Remarks - Anomoporia is morphological and phylogenetically closely related to Amylocorticium differing basically in the poroid hymenophore in the former (Pouzar 1966a).

Antella Miettinen, Ann. Bot. Fennici 53: 158, 2016.

Type species - Antrodiella niemelaei Vampola \&Vlasák, Czech Mycol. 63: 197, 2011.

Basidiome resupinate, hymenophore poroid, pores 1-4 per $\mathrm{mm}$, somewhat splitting to irpicoid, light coloured, yellowish when old. Hyphal system dimitic, with clamps, weakly to moderately cyanophilous, skeletal hyphae yellowish in masses, rather narrow, 2-3.5 $\mu \mathrm{m}$ in diam, with a distinct lumen. Gloeocystidia thin-walled, of variable sizes, well differentiated. Basidiospores ellipsoid, small (ab 4.5-2.5 $\mu \mathrm{m}$ ), thin-walled, smooth, negative in Melzer's reagent.

Remarks - Antella is a genus closely related to Antrodiella, differing in the presence of gloeocystidia. Also, skeletal hyphae in Antrodiella have an indistinct lumen and they are less cyanophilous (Miettinen \& Ryvarden 2016).

Anthoporia Karasiński \& Niemelä, Polish Bot. J. 61(1): 8, 2016.

Type species - Polyporus albobrunneus Romell, Ark. Bot. 11(no. 3): 10, 1911. 
Basidiome annual to perennial, resupinate, effused, soft, hymenophore poroid, with cottony subiculum and fimbriate margin, rhizomorphs present but inconspicuous. Hyphal system dimitic, generative hyphae with clamp connections, in subiculum and tube trama hyaline, in basal subiculum close to substrate brown, often with resinous or fine crystalline encrustation, and occasional simple-septa, skeletal hyphae thick-walled to almost solid, not reacting in $\mathrm{KOH}$, Melzer's reagent and Cotton Blue. Cystidia absent. Basidia clavate, with four sterigmata, and basal clamp. Basidiospores cylindrical to suballantoid, thin-walled, smooth, IKI-, CB-. Causing a brown rot.

Remarks - Anthoporia differs significantly from Antrodia in the short clavate basidia and shorter, narrower basidiospores. In addition, thick-walled hymenial cells, hyphidia or skeletocystidia are not observed in Anthoporia. The basidiomes of Anthoporia are totally resupinate, soft, with a cottony subiculum consisting of loosely arranged hyphae. Anthoporia differs from Antrodia in having brown generative hyphae in the upper subiculum (basal layer) next to the substrate and at the margin of the basidiome (Karasiński \& Niemelä 2016). Recently published molecular phylogenies have shown Polyporus albobrunneus to be only distantly related to Antrodia, Amyloporia, and Fibroporia, and this is also supported by the morphological characteristics indicated by Karasiński \& Niemelä (2016).

Antrodia P. Karst., Meddn Soc. Fauna Flora Fenn. 5: 40, 1879.

Type species - Polyporus serpens Fr., Observ. mycol. (Havniae) 2: 265, 1818.

Basidiome annual to perennial, resupinate to effused-reflexed, more rarely sessile and shelf like, mostly light coloured and tough to hard. Pores round to angular. Hyphal system dimitic, generative hyphae with clamps, often irregularly thickened, skeletal hyphae present, hyaline or slightly tinted in a few species, usually non-amyloid, variably amyloid in a few species (see Amyloporia). Cystidia not present. Cystidiols fusoid, small, ventricose, often present among the basidia. Basidia clavate, 4-stergimate, with a basal clamp. Basidiospores allantoid to oblongellipsoid, hyaline, thin-walled, smooth, negative in Melzer's reagent. Causing a brown rot, mostly in coniferous wood.

Remarks - Antrodia is a well know genus among the polypores with many species sharing the poroid hymenophore, dimitic hyphal system, basidiospores not reacting in Melzer's reagent, and causing a brown rot. Recently, some deviating species have been separated from the wide generic concept, and actually are accepted genera as Amyloporia and Fibroporia. Cartilosoma is a more controversial genus, and surely more phylogenetic studies are needed to assess a clear monophyly from Antrodia. Audet (2017g) described Resinoporia Audet. It differs from Antrodia s.str. or Amyloporia s.str. by abundant resinous matter occurs in the context and tubes, by tortuous and variably inflated skeletal hyphae, by basidia broadly clavate to barrel-shaped, with thickened walls after spore detachment. Resinoporia circumscribes the Androdia crassa group as selected by Spirin et al. (2015), but more studies are needed to elucidate relationships in the Antrodia crassa group. Anthoporia differs from Antrodia in having brown generative hyphae next to the substrate and at the margin of the basidiome. See also Rajchenberg et al. (2011) and Bernicchia \& Gorjón (2020).

Antrodiella Ryvarden \& I. Johans., Prelim. Polyp. Fl. E. Afr. (Oslo): 256, 1980.

Type species - Polyporus semisupinus Berk. \& M.A. Curtis, Grevillea 1(4): 50, 1872.

Basidiome resupinate to pileate, waxy soft when fresh, dense and hard and often semi translucent when dry, pileus if present, narrow and light-coloured, smooth to scrupose. Pore surface light ochraceous to straw-coloured when dry, pores entire and small, tubes concolorous with the pore surface, context white to pale straw-coloured. Hyphal system dimitic, generative hyphae with clamps, skeletal hyphae mostly narrow, hyaline thick-walled to solid, usually unbranched, more rarely with a few scattered branches. Cystidia absent or present. Basidia clavate, 4-sterigmate, with a basal clamp. Basidiospores small, rarely above $5 \mu \mathrm{m}$ in longest dimension, subglobose, ellipsoid to allantoid, thin-walled, hyaline and non-amyloid. On dead wood causing a white rot. 
Remarks - Antrodiella is above all characterized by the typical consistency of the fruitbody, being soft and dense, almost wax-like when fresh, becoming dense, hard, and semi translucent when dry (Ryvarden \& Johansen 1980).

Antrodiopsis Audet, Mushrooms nomenclatural novelties 1: 1, 2017.

Type species - Poria oleracea R.W. Davidson \& Lombard, Mycologia 39(3): 317, 1947.

Remarks - According to Audet (2017a), Antrodiopsis differs from Antrodia s.str. by basidiomata perennial, habitus only resupinate, smaller basidia and by uninucleate basidiospores.

Aphanobasidium Jülich, Persoonia 10(3): 326, 1979.

Type species - Corticium subnitens Bourdot \& Galzin, Hyménomyc. de France (Sceaux): 224, 1928.

Basidiome resupinate, effused, thin, ceraceous, light coloured, hymenophore smooth. Hyphal system monomitic, generative hyphae with clamps, hyaline, thin-walled. Cystidia lacking. Basidia (pleurobasidia) hyaline, small, thin-walled, with (2)4-sterigmata, with a basal clamp. Spores hyaline, ellipsoid, smooth, thin- or with slightly thickened walls, IKI-.

Remarks - Aphanobasidium includes a large and diverse group of pleurobasidiate corticioids species with smooth inamyloid basidiospores. Aphanobasidium is with Coronicium, Merulicium, and Radulomyces phylogenetically classified in the Pterulaceae Corner within the Agaricales (Larsson 2007b). Aphanobasidium basidiomes have a characteristic phenol smell when touched, a feature also shared with Pterula Fr. and Radulomyces (Olariaga, pers. obs.).

Aquascypha D.A. Reid, Nova Hedwigia, Beih. 18: 51, 1965.

Type species - Stereum hydrophorum Berk., Ann. Mag. nat. Hist., Ser. 1 14: 327, 1844.

Basidiome coriaceous, centrally stipitate, infundibuliform, often coalescent, pileus minutely tomentose or velutinate, concentrically zoned, varying in colour from dark brown to dark purplebrown, normally bearing conspicuous flattened dendroid or antler-like processes, hymenial surface smooth, distinctly pruinose, ochraceous-buff near the margin but becoming almost white towards the stipe in well preserved specimens. Stipe relatively short, minutely velutinate, dark brown to dark purple brown, arising from a well-developed basal disc of mycelium. Flesh of the pileus creamy-white, becoming dark brown in the region of the hymenium. Hyphal system trimitic, consisting of thin-walled, hyaline, branched generative hyphae, which bear inconspicuous clampconnexions at the septa; thick-walled, unbranched skeletal hyphae which are distinctly brownish in colour; and thick-walled, pale brownish or hyaline, branched binding hyphae, lacking clamp connexions and in which the branches are of limited growth. Cystidia and gloeocystidia absent. Basidia not seen. Basidiospores hyaline, smooth, globose or subglobose, cylindrical (according to Ryvarden 2010).

Remarks - Aquascypha is known from tropical South America. It is a characteristic fungus with infundibuliform basidiomes growing on dead wood or debris. Phylogenetic relationships of Aquascypha remain unclear (Sjökvist et al. 2012).

Aquathanatephorus C.C. Tu \& Kimbr., Bot. Gaz. 139: 459, 1978

Type species - Aquathanatephorus pendulus C.C. Tu \& Kimbr., Bot. Gaz. 39(4): 459, 1978.

Remarks - A synonym of Thanatephorus Donk.

Arrasia Bernicchia, Gorjón \& Nakasone, Mycotaxon 118: 258, 2011.

Type species - Arrasia rostrata Bernicchia, Gorjón \& Nakasone, Mycotaxon 118: 258, 2011.

Basidiome effuse, adnate, thin, white, smooth, finely farinaceous, with distinct margin. Hyphal system monomitic, hyphae clamped. Dendrohyphidia filamentous, branched, clamped. Basidia suburniform at first, then flexuous, clavate to obclavate, with 4-sterigmata. sometimes with a basal lobe, clamped. Basidiospores broadly subfusiform to biapiculate, distal end elongating into a thick-walled rostrum, walls hyaline, slightly thick, smooth, cyanophilous, IKI-. 
Remarks - Arrasia is well characterized by the large thick-walled basidiospores developing a distal rostrum when mature (Bernicchia et al. 2011). It seems to be related to dendrotheloid fungi such as Dendrothele by sharing the same ecology inhabiting bark of living trees, crustose basidioma, suburniform basidia at first, and structure of the hymenium with many dendrophyphidia and abundant crystalline deposits as an adaptation to dry and exposure. Monotypic genus.

Asterodon Pat., Bull. Soc. mycol. Fr. 9:53, 1894.

Type species - Asterodon ferruginosus Pat., [as 'ferruginosum'], Bull. Soc. mycol. Fr. 10(3): 130, 1894.

Basidiome annual, resupinate, effused, margin floccose, hymenophore hydnoid. Hyphal system dimitic, generative hyphae simple-septate, hyaline to pale brown, skeletal hyphae unbranched, or rarely bifurcate, aseptate, with smooth, brownish ochraceous and thickened walls. Hymenial setae and asterosetae present. Basidia clavate, with 4-sterigmata, simple-septate at the base. Basidiospores ellipsoid, hyaline, smooth, thin-walled, IKI-.

Remarks - Asterodon is related to Hymenochaete and it belongs to the Hymenochaetaceae Donk (Larsson 2007b). It differs from Hymenochaete mainly in the branched asterosetae.

Asterostroma Massee, J. Linn. Soc., Bot. 25: 154, 1889.

Type species - Corticium apalum Berk. \& Broome, J. Linn. Soc., Bot. 14: 72, 1875.

Basidiome resupinate, effused, membranaceous to pellicular, brittle, loosely adnate, hymenophore smooth, cream to ochraceous. Hyphal system dimitic with simple-septate generative hyphae and asterosetae. Gloeocystidia present. Basidia utriform, with 4-sterigmata and with a simple basal septum. Basidiospores subglobose to ellipsoid, smooth or tuberculate, amyloid (some species with inamyloid basidiospores, subg. Austroasterostroma Parmasto).

Remarks - Asterostroma seems to be related to Asterodon sharing the same type of asterosetae but differing in the type of basidiospores and hymenophore morphology. Phylogenetic analysis includes Asterostroma in the russuloid clade and Asterodon in the hymenochaetoid clade. Asterostroma is included, with Vararia, Dichostereum, and Scytinostroma in the Peniophoraceae Lotsy. The phylogenetic analysis shows that dextrinoid hyphidia do not define a monophyletic group (Larsson 2007b). For a survey of Asterostroma see also Boidin et al. (1997b).

Asterostromella Höhn. \& Litsch., Sber. Akad. Wiss. Wien, Math.-naturw. Kl., Abt. 1 116: 773, 1907.

Type species - Corticium epiphyllum ss Ravenel.

Remarks - A synonym of Vararia P. Karst. A name based on a misapplied type: Corticium epiphyllum Pers. belongs to Athelia and Corticium epiphyllum sensu Ravenel is Vararia phyllophila (Massee) D.P. Rogers \& H.S. Jacks.

Athelia Pers., Mycol. eur. (Erlanga) 1: 83, 1822.

Type species - Athelia epiphylla Pers., Mycol. eur. (Erlanga) 1: 84, 1822.

Basidiome resupinate, thin, pellicular (athelioid), easily detachable, white to whitish, hymenophore smooth. Hyphal system monomitic, hyphae with or without clamps, hyaline, thinwalled, loosely interwoven. Cystidia usually absent, some species with leptocystidia. Basidia short, more or less clavate, in clusters from the subhymenial hyphae, 2-4-sterigmata, clamped or with a simple septum. Basidiospores subglobose to ellipsoid or cylindrical, smooth, thin-walled, often glued together, IKI-.

Remarks - Athelia is a large but well delimited genus by the ephemeral and pellicular basidiome and the hymenial structure with open branched hyphae arranged in candelabrum-like clusters. It includes usually acystidiate species, but cystidial elements may be present in few species. According to Larsson et al. (2004), Athelia seems to be related to Piloderma, Tylospora, Byssocorticium, Atheliopsis, and Amphinema. The athelioid clade could be related to the euagarics clade by one subclade in which Amylocorticium, Ceraceomyces tessulatus, Hypochniciellum, and 
Anomoporia are present. The athelioid clade includes mycorrhizal species like Piloderma, Amphinema, and Tylospora. A mycorrhizal habit has not been observed in Athelia and Atheliopsis, but Oberwinkler (1970) described the parasitism by A. epiphylla, on unicellular green algae, and by A. arachnoidea (Berk.) Jülich on lichens.

Atheliachaete Spirin \& Zmitr., Biodiversity of the Heterobasidiomycetes and non-gilled Hymenomycetes (former Aphyllophorales) of Israel: 456, 2011.

Type species - Thelephora sanguinea Fr., Elenchus Fungorum 1: 203, 1828.

Remarks - Atheliachaete was described as substitute of the superfluous name Leptochaete Zmitr. \& Spirin. It can be considered as a synonym of Phanerochaete P. Karst. sensu lato.

Athelicium K.H. Larss. \& Hjortstam, Windahlia 15: 49, 1986.

Type species - Athelicium stridii K.H. Larss. \& Hjortstam, in Hjortstam \& Larsson, Windahlia 15: 51, 1986.

Basidiome resupinate, loosely adnate, hymenophore smooth. Hyphal system monomitic, hyphae with clamps or simple-septate. Cystidia absent. Basidia constricted, more or less pedunculate. Basidiospores ellipsoid to amygdaliform, smooth, thin to thick-walled, IKI-.

Remarks - Athelicium is characterized by the small basidiome growing in dry and exposed places, and microscopically by the large basidia and basidiospores. Phylogenetic analyses associate Athelicium with Lichenomphalia Redhead, Lutzoni, Moncalvo \& Vilgalys and Arrhenia Fr. species and with Athelia pyriformis (M.P. Christ.) Jülich in the Hygrophoraceae Lotsy (Larsson 2007b).

Athelidium Oberw., Sydowia 19: 62, 1965.

Type species - Xenasma aurantiacum M.P. Christ., Dansk bot. Ark. 19(2): 107, 1960.

Basidiome resupinate, effused, hypochnoid to membranaceous. Hyphal system monomitic, hyphae without clamps, thin-walled. Basidia more or less constricted, cylindrical to clavate, 4sterigmata, with a simple basal septum. Basidiospores hyaline, thin-walled, smooth, non-amyloid.

Remarks - Athelidium is a monotypic genus phylogenetically related to Cristinia and Lindtneria in the Stephanosporaceae Oberw. \& E. Horak; this relationship is unclear because morphologically it shares only the yellow-orange colour of the basidioma (Larsson 2007b).

Athelocystis Hjortstam \& Ryvarden, Syn. Fung. (Oslo) 27: 20, 2010.

Type species - Athelocystis capitata Hjortstam \& Ryvarden, Syn. Fung. (Oslo) 27: 20, 2010.

Basidiome resupinate, effused, closely adnate, membranous, hymenophore smooth, whitish, then cream-colored, margin fibrillose. Hyphal system monomitic, hyphae with simple-septa, thinwalled. Cystidia tubular with a capitate apex, thin-walled, apex strongly encrusted with yellowish amorphous matter. Basidia sphaeropedunculate, with 4-sterigmatea, with a basal simple septum. Basidiospores narrowly ellipsoid to pip-shaped, smooth, thin-walled, IKI-, CB-.

Remarks - Athelocystis is somewhat reminiscent of Athelopsis by the pedunculate basidia. Monotypic genus known only from Brazil (Hjortstam \& Ryvarden 2010a).

Atheloderma Parmasto, Consp. System. Corticiac. (Tartu): 73, 1968.

Type species - Atheloderma mirabile Parmasto, Consp. System. Corticiac. (Tartu): 200, 1968.

Basidiome resupinate, effused, pellicular, hymenophore smooth, margin more or less fimbriate or with rhizomorphs. Hyphal system monomitic, hyphae with clamps. Cystidia cylindrical, often with a clamped septum, thin-walled, not encrusted. Basidia clavate, constricted, with 4-sterigmata, and a basal clamp. Basidiospores ellipsoid to cylindrical, smooth, thin-walled, inamyloid, indextrinoid.

Remarks - Atheloderma reminds Hyphoderma in basidia and basidiospores but its relationships are not clear. Atheloderma is considered a synonym of Hyphoderma by Jülich (1974). Molecular data include Atheloderma in the Rickenella family with other genera such as 
Globulicium, Cyphellostereum, and Resinicium but the delimitation of this family is unclear (Larsson 2007b).

Athelopsis Oberw. ex Parmasto, Consp. System. Corticiac. (Tartu): 41, 1968.

Type species - Corticium glaucinum Bourdot \& Galzin, Hyménomyc. de France (Sceaux): 207, 1928.

Basidiome resupinate, thin, pellicular; hymenophore smooth, usually with yellowish tints. Hyphal system monomitic; hyphae with clamps at all septa, thin-walled, hyaline. Cystidia absent, cystidiols may be present. Basidia clavate, stalked, with 4-sterigmata, and with a basal clamp. Basidiospores ellipsoid to cylindrical, smooth, thin-walled, hyaline, IKI-.

Remarks - Athelopsis is characterized by the pellicular basidiome, usually with yellowish or greenish tints and by the stalked basidia. Basidia are arranged in a compacted layer that remind those of Athelia where basidia are arranged in more open and branched candelabrum-like clusters. According to the molecular studies by Larsson et al. (2004), Athelopsis forms part of the athelioid clade, close to Athelia and Piloderma and closely related to Byssocorticium. Larsson (2007b) confirms these results including Athelopsis in Atheliaceae and indicating that molecular data show that Athelia and Athelopsis are polyphyletic.

Atraporiella Ryvarden, Syn. Fung. (Oslo) 23: 38, 2007.

Type species - Atraporiella neotropica Ryvarden, Syn. Fung. (Oslo) 23: 38, 2007.

Basidiome resupinate, annual, small, easily separable, soft and slightly waxy when fresh, brittle and contracting when drying and then disc like with raised loosened margin, resinous brittle when dry, pore surface whitish when fresh and actively growing, very rapidly stained dark brown to black when touched, pale ochraceous when dry except for stained parts, pores angular, subiculum thin to almost invisible, pale brown and cottony. Hyphal system monomitic, generative hyphae with clamps, pale brown, thin-walled, richly branched. Cystidia and other sterile hymenial elements absent. Basidia clavate, 4-sterigmate, with a basal clamp. Basidiospores ellipsoid, thin-walled, smooth, hyaline, IKI-. Presumably causing a white rot.

Remarks - Atraporiella is characterized by the white pore surface becoming brown to black when touched. Nigroporus Murrill is characterized by being deep blackish violet besides having a distinct dimitic hyphal system (Ryvarden 2007). According to Wu et al. (2017), Atraporiella is placed in the residual polyporoid clade as a monophyletic lineage, with Antrodiella, Pouzaroporia, Steccherinum, and Xanthoporus.

Aurantiporus Murrill, Bull. Torrey bot. Club 32(9): 487, 1905.

Type species - Polyporus pilotae Schwein., Trans. Am. phil. Soc., New Series 4(2): 156, 1832.

Basidiome resupinate to pileate, white to pink or orange, unchanging to red in $\mathrm{KOH}$, hymenophore tubular with round to angular pores. Hyphal system monomitic, generative hyphae hyaline, with clamps, thin-walled, encrusted or not, gloeoplerous hyphae may be present. Cystidia and cystidioles absent. Basidia clavate, with a basal clamp, 4-sterigmatic. Basidiospores ellipsoid, hyaline, smooth, thin-walled, IKI-, CB-. Causing a white rot.

Remarks - Aurantiporus is distinguished among the monomitic polypores by the pore surface usually pinkish to salmon color or bright orange, watery, cartilaginous consistency, and the agglutination of the hyphae. Zmitrovich (2018) proposed the genus Pappia Zmitr. to accommodate Polyporus fissilis Berk. \& M.A. Curtis.

Auriculariopsis Maire, Bull. Soc. mycol. Fr. 18(suppl.):102, 1902.

Type species - Cyphella ampla Lév., Annls Sci. Nat., Bot., sér. 3, 9: 126, 1848.

Basidiome cupulate, abhymenial surface velvety to tomentose, hymenophore folded, subgelatinosus when fresh. Hyphal system monomitic to dimitic, generative hyphae with clamps 
and hair skeletal hyphae close to the abhymenial surface. Cystidia absent. Basidia subclavate, with 4-sterigmata and a basal clamp. Basidiospores allantoid, smooth, thin-walled, IKI-.

Remarks - Auriculariopsis is characterized by the resupinate to cupulate basidiome and microscopically by the absence of cystidia and by the smooth inamyloid basidiospores (Maire 1902). Auriculariopsis is phylogenetically related to Schizophyllum and Nakasone (1996) placed Cyphella ampla, the generic type in synonymy with Schizophyllum. Auriculariopsis albomellea (Bondartsev) Kotl., the other species of the genus, was regarded as closely related to Phlebia albida H. Post and transferred to Phlebia (Nakasone 1996).

Auriporia Ryvarden, Norw. J. Bot. 20: 3, 1973.

Type species - Poria aurea Peck, Ann. Rep. Reg. N.Y. St. Mus. 43: 67, 1890.

Basidiome resupinate to pileate, annual, pore surface yellow to white. Hyphal system monoto dimitic, generative hyphae with scattered clamps, skeletal hyphae, when present, few, mostly confined to the context. Cystidia ventricose, thick-walled, apically encrusted. Basidia clavate, with 4-sterigmate, with a basal clamp. Basidiospores cylindrical to ellipsoid, smooth, thin-walled, negative in Melzer's reagent. On both hardwoods and conifers, causing a brown rot.

Remarks - Auriporia is morphologically related to Antrodia by the type of rot and hyphal system but differing by the prominent cystidia (Ryvarden 1973).

Auriscalpium Gray, Nat. Arr. Brit. Pl. (London) 1: 650, 1821.

Type species - Auriscalpium vulgare Gray, Nat. Arr. Brit. Pl. (London) 1: 650, 1821.

Basidiome stipitate and pileate, hymenophore hydnoid, upper sterile surface hirsute. Hyphal system dimitic, generative hyphae with clamps, skeletal hyphae present in the context. Gloeocystidia present. Basidia subclavate, with 4-sterigmata, and a basal clamp. Basidiospores ellipsoid, ornamented, amyloid.

Remarks - Auriscalpium is a stipitate hydnoid genus, although it does not share the corticioid habit we include it in this manual to highlight the variability of the basidiome structure present within the russuloid lineage. It is phylogenetically very closely related to Gloiodon, with effusereflexed basidiome and to Dentipratulum, with hydnoid gregarious basidiomes (Larsson \& Larsson 2003, Larsson 2007b).

Australicium Hjorstam \& Ryvarden, Synopsis Fungorum 15: 19, 2002.

Type species - Corticium singulare G. Cunn., Trans. R. Soc. New Zealand 82: 325, 1954.

Basidiome resupinate, adnate, hymenophore smooth, usually pale brown, subiculum distinct, brownish to brown, tomentous. Hyphal system monomitic, subicular hyphae thick-walled, yellowish to brownish, with simple-septa even single or double scattered clamps may occur, subhymenial hyphae hyaline, thin or with thickened walls, simple-septate. Cystidia or hyphal ends rare, hyaline, thin-walled, not encrusted. Basidia subclavate, slightly constricted, with 4-sterigmata, with a simple basal clamp. Basidiospores ellipsoid to cylindrical, smooth, thin-walled, hyaline, IKI-, CB-.

Remarks - Australicium was created to accommodate Corticium singulare, a species with a distinct brown and tomentose subiculum composed of brownish thick-walled hyphae (Hjortstam \& Ryvarden 2002b). It is closely related to Phanerochaete but differing morphologically in the brown subiculum.

Australohydnum Jülich, Persoonia 10: 138, 1978.

Type species - Hydnum griseofuscescens Reichardt, Verh. zool.-bot. Ges. Wien 16: 374, 1866. (=Corticium dregeanum Berk, J. Bot., Lond. 5: 3, 1846).

Basidiome annual, resupinate to effuse-reflexed, membranaceous, adnate, context homogeneous, margin determinate, rhizomorphs or hyphal strands lacking. Hymenophore hydnoid. Hyphal system monomitic or indistinctly dimitic, hyphae simple-septate, hyaline, cylindrical, compactly arranged, thin-walled in the subhymenium, thick-walled in the trama, smooth. Cystidia 
(skeletocystidia) present, abundant, arising from thick-walled, clampless, generative hyphae, the projecting part loosely encrusted. Basidia clavate, with a simple basal septum. Basidiospores hyaline, more or less ellipsoid, thin-walled, smooth, not amyloid.

Remarks - Australohydnum is phylogenetically related to phanerochaetoid fungi as Phanerochaete and Phlebiopsis (Larsson 2007b). It shares with these genera the generative simpleseptate hyphae, but it differs in the pseudodimitic hyphal system and in the presence of skeletocystidia.

Australovuilleminia Ghobad-Nejhad \& Hallenb., in Ghobad-Nejhad et al. Taxon 59(5): 1528, 2010.

Type species - Australovuilleminia coccinea Ghobad-Nejhad \& Hallenb., in Ghobad-Nejhad et al. Taxon 59(5): 1529, 2010.

Basidiome resupinate, decorticant, erumpent, closely adnate, reddish orange to coccine red, subgelatinous, margin indistinct. Hyphal system monomitic, hyphae with clamps, encrusted with reddish-orange matter, thin to thick-walled. Subiculum thin, composed of highly branched, irregularly arranged hyphae. Cystidia cylindrical, considerably thick-walled excepting in the apical part, contents homogeneous, yellowish to orange brown, not reacting in sulphovainilline neither in Melzer's reagent, slightly or conspicuously projecting above the basidial layer. Dendrohyphidia and hyphidia abundant. Basidia first tubular, becoming elongate, long clavate, flexuous, with 4sterigmata, densely arranged in the hymenium and intermixed with numerous hyphidia and dendrohyphidia, with a basal clamp. Basidiospores allantoid, thin-walled, smooth, IKI-, cyanophilous.

Remarks - Australovuilleminia resembles Vuilleminia in its decorticating habit, presence of dendrohyphidia, large basidia and large basidiospores but differing in the well-developed basidial layer in a true hymenium and a distinguishable subiculum. Also, the striking red colour of the basidiome is diagnostic (Ghobad-Nejhad et al. 2010). Australovuilleminia is the austral counterpart of Vuilleminia and both genera are also phylogenetically closely related.

Baltazaria Leal-Dutra, Dentinger \& G.W. Griff., in Leal-Dutra, Neves, Griffith, Reck, Clasen \& Dentinger, MycoKeys 37: 47, 2018. 1851.

Type species - Thelephora galactina Fr., Nova Acta R. Soc. Scient. upsal., Ser. 3 1(1): 136,

Basidiome corticioid, adherent to effused, coriaceous/membranate when fresh, hard when dry, usually white, cream, or pale ochraceous. Context densely homogeneous with thick-walled and dextrinoid skeletal-binding hyphae, sometimes bearing rows of short papillae or skeletodendrohyphidia. Global distribution.

Remarks - Baltazaria is related to Scytinostroma in the Lachnocladiaceae. See Leal-Dutra (2018) for further comments.

Basidioradulum Nobles, Mycologia 59: 192, 1967.

Type species - Hydnum radula Fr., Observ. mycol. (Havniae) 2: 271, 1818.

Basidiome resupinate to effuse-reflexed, hymenophore hydnoid to irpicoid. Hyphal system monomitic, hyphae with clamps. Cystidia cylindrical, not encrusted. Basidia cylindrical, more or less constricted, with 4-sterigmata, with a basal clamp. Basidiospores cylindrical, smooth, thinwalled, IKI-.

Remarks - Basidioradulum is closely related to Hyphoderma but mainly distinguished by its irpicoid hymenophore (Nobles 1967). Hymenophore configuration is not considered a reliable character for segregating species in different genera (e.g. Hyphodontia with smooth and odontioid species; Mycoacia species all considered under Phlebia), but according to available molecular data (Wagner \& Fischer 2002, Larsson 2007b) the generic relationships of Basidioradulum remains still unclear and for the time present, we regard it different from Hyphoderma. 
Beccariella Ces., Atti Accad. Sci. fis. mat. Napoli 8(8): 9, 1879.

Type species - Beccariella insignis Ces., Atti Accad. Sci. fis. mat. Napoli 8(8): 10, 1879.

Remarks - A synonym of Cymatoderma Jungh.

Bjerkandera P. Karst., Meddn Soc. Fauna Flora fenn. 5: 38, 1879.

Type species - Boletus adustus Willd., Fl. berol. Prodr.: 392, 1787.

Basidiome annual, effused, resupinate to pileate, pileus light-coloured, azonate, finely velutinous to pubescent. Pore surface blackish to brown, distinctly delimited towards a white to pale cream context. Hyphal system monomitic, hyphae with clamps, thin-walled hyphae in the hymenium, more thick-walled to almost solid in the context and pileus. Cystidia none. Basidia clavate, 4-sterigmata, with a basal clamp. Basidiospores oblong ellipsoid, hyaline, smooth, thinwalled and non-amyloid. Causing a white rot.

Remarks - Bjerkandera is easily to recognize in the field by the large effuse-reflexed basidiomes and the greyish to blackish colour of the tube layer with violet shadows. The monomitic hyphal system, smooth basidiospores, and type of rot points a relationship with Tyromyces. See Ryvarden \& Melo (2017) and Bernicchia \& Gorjón (2020).

Boidinia Stalpers \& Hjortstam, in Hjortstam \& Stalpers, Mycotaxon 14: 76, 1982.

Type species - Hypochnus furfuraceus Bres., Fung. trident. 2(11-13): 97, 1900.

Basidiome annual, resupinate, separable, thin, porulose or furfuraceus, sometimes pellicular to soft membranaceous. Hymenophore smooth, white to cream-coloured, often becoming pale ochraceous or alutaceous with age, margin indistinct. Hyphal system monomitic. Hyphae with clamps, thin- to thick-walled. Gloeocystidia tubular to subclavate, more or less sinuous, basally somewhat ventricose, hyaline or with yellowish contents, SA+ (sometimes weakly), not or slightly projecting above the basidia. Basidia suburniform, usually terminal, but some lateral basidia may occur, often with 1-2 adventitious septa. Basidiospores hyaline, thin- to somewhat thick-walled, globose or subglobose, echinulate or verrucose, ornamentation disappearing in $\mathrm{KOH}$, strongly amyloid, with distinct apiculus.

Remarks - Boidinia is morphologically characterized by the amyloid ornamented basidiospores, usually subglobose, and by the suburniform basidia (Hjortstam \& Stalpers 1982). Boidinia and Gloeopeniophorella belong to the Russulaceae Lotsy. Contrary to Lactarius and Russula that are mycorrhizal, Boidinia is saprobic and it clusters in a basal position in the clade, suggesting that the ancestor in the russuloid clade to the agaricoid forms had a corticioid basidiome and was saprotrophic (Larsson \& Larsson 2003).

Boidinella Nakasone, Crytogamie, Mycologie 32(2): 192, 2011.

Type species - Dendrothele globulispora Boidin \& Lanq., Bull. Soc. Mycol. France 112: 103, 1996.

Basidiome resupinate, effuse, thin, soft, fragile, smooth, densely farinaceous to membranous, margin more or less abrupt. Hyphal system monomitic with clamped hyphae. Dendrohyphidia delicate. Leptocystidia obclavate, attenuate, sometimes moniliform. Basidia urniform, oleaginous, walls cyanophilous, 4-sterigmate. Basidiospores subspherical to ellipsoid, walls hyaline, smooth, slightly thick, cyanophilous, not amyloid. On dead stems of monocots.

Remarks - Boidinella is characterized by the effuse, soft, densely farinaceous or membranous basidiomes, urniform basidia with 4 sterigmata, obclavate leptocystidia, dendrohyphidia, and basidiospores with smooth, slightly thickened, cyanophilous walls. Boidinella is morphologically similar to Dendrothele but differing in the delicate and fragile nature of dendrohyphidia, cystidia, and basidia in the former. Boidinella lacks crystals and inhabits dead stems of monocots. Basidiospores in Sistotremella have also cyanophilous walls but are much smaller and basidia always have 6-8 sterigmata. Leptocorticium also shows similarities to Boidinella, but basidiospore walls are thin and not cyanophilous in the former. For further comments see Nakasone (2011). 
Bonia Pat., Bull. Soc. mycol. Fr. 8(2): 48, 1892.

Type species - Bonia papyrina Pat., Bull. Soc. mycol. Fr. 8(2): 48, 1892.

Remarks - A latter homonym of Bonia Balansa 1890 (Gramineae). Bonia papyrina was later considered a synonym of Heterochaete tenuicula (Lév.) Pat. which is a synonym of Heterochaete delicata (Klotzsch ex. Berk.) Bres. (Tremellales).

Boninohydnum S. Ito \& S. Imai, Trans. Sapporo Nat. Hist. Soc. 16: 127, 1940.

Type species - Boninohydnum pini S. Ito \& S. Imai, Trans. Sapporo Nat. Hist. Soc. 16: 127, 1940.

Remarks - A synonym of Gyrodontium Pat.

Boreostereum Parmasto, Consp. System. Corticiac. (Tartu): 186, 1968.

Type species - Stereum radiatum Peck, Bull. Buffalo Soc. nat. Sci. 1: 62, 1873.

Basidiome annual, resupinate to effuse-reflexed, soft-coriaceous to brittle; upper sterile surface dark brown to black; hymenial surface even to radially and concentrically folded, brownish orange. Hyphal system dimitic, generative hyphae without clamps, hyaline to pale brown; skeletal hyphae greenish in $\mathrm{KOH}$. Hyphidia acute. Cystidia absent, hyphoid hyphal ends present. Basidia subclavate to subcylindrical, with 4-sterigmata, and with a simple basal septum. Basidiospores cylindrical, hyaline to yellowish; smooth, thin-walled, IKI-.

Remarks - Boreostereum is characterized by the dimitic hyphal system with hyphae and cystidiols having characteristic brown encrustation that turns greenish in potassium hydroxide (Parmasto 1968). Boreostereum belongs to the Gloeophyllaceae, with Veluticeps and Chaetodermella as the closest related genera. The species within this family are saprobics and associated with a brown rot (Larsson 2007b).

Botryobasidium Donk, Medded. Nedl. Mycol. Ver. 18-20: 116, 1931.

Type species - Corticium subcoronatum Höhn. \& Litsch., Sber. Akad. Wiss. Wien, Math.naturw. Kl., Abt. 1 116: 822 ,1907.

Synonym - Botryohypochnus Donk.

Basidiome resupinate, thin, floccose, reticulate, arachnoid, hypochnoid to porulose under the lens without forming a continuous hymenium. Hyphal system monomitic, hyphae broad (usually 8$10 \mu \mathrm{m}$ wide or more), loosely interwoven, branched at right angles, basal hyphae more or less thick-walled, clamps present or absent. Cystidia absent or present. Basidia subcylindrical to cylindrical, in clusters on hymenial hyphal branches, with (4)6-8-sterigmata. Basidiospores variable, usually ellipsoid, allantoid or navicular, smooth or ornamented, IKI-, without secondary basidiospores by repetition.

Remarks - Botryobasidium is a well-defined genus characterized by its not continuous arachnoid to porulose basidiome, and microscopically by the very broad hyphae branched at right angles, and the subcylindrical basidia with usually 6-8 sterigmata. Botryobasidium shows clear relations with Thanatephorus, Ceratobasidium, and Cejpomyces in which basidiospores usually grow by repetition. It is also related to Sistotrema which differs in the nature of the hymenium (palisade in Sistotrema and clustered in Botryobasidium) and shape of basidia (urniform in Sistotrema and cylindrical in Botryobasidium). Many species in Botryobasidium have known anamorphs that are useful in distinguishing related taxa. Molecular data have confirmed these relations, placing Botryobasidium in the cantharelloid clade, with Cantharellus, Craterellus, Hydnum, and Clavulina (Hibbett et al. 1997, Moncalvo et al. 2006). Botryohypochnus differs from other Botryobasidium species by the distinctly ornamented and tetrasporic basidia; Langer (1994b) synonymized both genera and treated as a whole group smooth and ornamented spored species. Some molecular studies (cf. Binder et al. 2005, Moncalvo et al. 2006) show Botryohypochnus closely related to Botryobasidium in a strongly supported monophyletic clade.

Botryodontia (Hjortstam \& Ryvarden) Hjortstam, Mycotaxon 28(1): 20, 1987. 
Basionym - Candelabrochaete subgen. Botryodontia Hjortstam \& Ryvarden, Mycotaxon 25 (2): 545, 1986. 1986.

Type species - Candelabrochaete cirrata Hjortstam \& Ryvarden, Mycotaxon 25(2): 545,

Basidiome resupinate, loosely adnate, fairly thin, arachnoid or distinctly continuous, tufted and with small to relatively small aculei. Hyphal cords conspicuous in the type. Hyphal system monomitic or dimitic-duplex in the type, hyphae thin to distinctly thick-walled, without clamps. Cystidia or hyphoid elements present, smooth or strongly encrusted. Basidia short, about 10-15(20) $\mu \mathrm{m}$, obconical to becoming short-cylindrical, with 4 sterigmata and without basal clamp. Basidiospores about 6-7 $\mu \mathrm{m}$ in diam., subglobose, thin-walled or with slight thickened walls, smooth, IKI-, CB-.

Remarks - Botryodontia was proposed to accommodate Candelabrochaete cirrata which differs from other species in the genus by its shorter, mostly obconical to short-cylindrical basidia (Hjortstam 1987b). Botryodontia is recognized microscopically by possessing hyphae without clamp-connections, poorly differentiated, smooth or encrusted cystidia which occur mainly in the aculei, short, obconical basidia, usually 10-15 $\mu \mathrm{m}$ long, with four sterigmata, and subglobose, thinwalled, and smooth basidiospores. Macroscopically, it is reminiscent of many Hyphodontia species in having a colliculose to odontioid hymenophore. The main difference between unclamped Hyphodontia species and Botryodontia lies in the morphology of the basidia. In Hyphodontia these are large, subclavate, and with a median constriction, whereas in Botryodontia they are short and obconical. According to Sell et al. (2014) Oxyporus philadelphi (Parmasto) Ryvarden and Botryodontia millavensis (Bourdot \& Galzin) Duhem \& H. Michel are conspecific, as suggested by their similar morphology and ITS sequences. The species is closely related to the type species ( $B$. cirrata) of the genus Botryodontia, and therefore the name B. millavensis should currently be used for this taxon.

Botryohypochnus Donk, Medded. Nedl. Mycol. Ver. 18-20: 118, 1931.

Type species - Hypochnus isabellinus Fr., Observ. mycol. (Havniae) 2: 281, 1818.

Remarks - A synonym of Botryobasidium Donk. It has long been used for those species of Botryobasidium with aculeate basidiospores. Phylogenetic analyses show that both genera are closely related in a monophyletic clade (Moncalvo et al. 2006).

Bourdotiella Duhem \& Schultheis, Cryptogamie, Mycol. 32(4): 392, 2011.

Type species - Bourdotiella complicata Duhem \& Schultheis, Cryptogamie, Mycol. 32(4): 393, 2011.

Basidiome resupinate, effused, hymenophore odontioid with irregular protuberances with penicillate apex composed of densely encrusted hyphae, margin thinning out or indifferentiate. Hyphal system monomitic, hyphae with clamps, hyphal segment irregular and somewhat tortuose and inflated, slightly thick-walled in subhymenium. Dendrophyses poorly differentiated. Cystidia absent. Basidia utriform, with 4-sterigmata, up to $30 \mu \mathrm{m}$ long, with a basal clamp. Basidiospores ellipsoid, smooth, thin-walled, IKI-, CB-, guttulate.

Remarks - Bourdotiella is morphologically closely related to Dentocorticium, the latter differing in the long clavate basidia (more than $30 \mu \mathrm{m}$ long) and conspicuous, well developed dendrophyses. Also, there are similarities with Hyphodontia s.l., by the odontioid hymenophore and utriform basidia, but Bourdotiella differs in the presence of dendrophyses and somewhat irregularly inflated hyphae (Duhem \& Schultheis 2011).

Brevicellicium K.H. Larss. \& Hjortstam, in Hjortstam \& Larsson, Mycotaxon 7: 117, 1978.

Type species - Corticium exile H.S. Jacks., Can. J. Res., Section C 28: 721, 1950.

Basidiome resupinate, adnate, effused, membranaceous to ceraceous, hymenophore smooth to granulose. Hyphal system monomitic, hyphae clamped, hyaline and distinctly isodiametric. 
Cystidia absent, sphaerocysts can be present. Basidia shortly cylindrical, with 4-sterigmata and a basal clamp. Basidiospores subglobose to ellipsoid, smooth, thin-walled, IKI-, CB-.

Remarks - Brevicellicium is above all characterized by the short-celled and isodiametric inflated hyphae and by the small smooth basidiospores (Hjortstam \& Larsson 1978). It reminds in some respects to Trechispora, but it lacks the ampullate hyphae and the basidiome is usually more structured and not fragile as in Trechispora. According to molecular data, Brevicellicium cluster with other trechisporoid fungi as Trechispora, Subulicystidium, or Tubulicium. (Larsson 2007b).

Brevicellopsis Hjortstam \& Ryvarden, Synopsis Fungorum 25: 15, 2008. 1980.

Type species - Brevicellicium allantosporum Hjortstam \& Ryvarden, Mycotaxon 12: 170,

Basidiome resupinate, loosely adnate, soft and fragile, hymenophore distinctly odontioid with small almost subulate aculei. Hyphal system monomitic, basal hyphae thin to moderately thickwalled, subhymenial hyphae thin-walled, with an isodiametric appearance, all hyphae with clamps. Cystidia absent. Basidia clavate, with 4-sterigmata, with a basal clamp. Basidiospores reniform to allantoid, smooth, thin-walled, IKI-, CB-.

Remarks - Brevicellopsis reminds Brevicellicium by the isodiametric subhymenial cells, but in the latter the hymenophore is smooth and basidiospores are usually rhomboid to ellipsoid, whereas in Brevicellopsis the hymenial surface is odontioid and the basidiospores are narrowly allantoid (Hjortstam \& Ryvarden 2008).

Brunneocorticium Sheng H. Wu, Mycologia 99(2): 303, 2007.

Type species - Brunneocorticium pyriforme Sheng H. Wu, Mycologia 99(2): 306, 2007 (=Dendrothele bispora Burds. \& Nakasone, Mycotaxon 17: 253, 1983).

Basidiome resupinate, hymenial surface smooth, cracked, whitish, margin brown. Hyphal system dimitic, generative hyphae with clamps, subiculum brown due to the presence of abundant yellowish-brown skeletal hyphae. Dendrohyphidia present. Leptocystidia with some secondary simple septa present but often difficult to detect. Basidia subclavate, with a median constriction, with two sterigmata, and a basal clamp. Basidiospores pyriform, apiculate, smooth, thin-walled, guttulate, hyaline, IKI-, CB-.

Remarks - Brunneocorticium is characterized by the dimitic hyphal system with brownish skeletal hyphae, presence of dendrohyphidia and leptocystidia with secondary simple-septa, bisterigmate basidia, and pyriform basidiospores (Wu et al. 2007). Brunneocorticium belongs to the agaricoid clade. See Nakasone et al. (2009) for further comments.

Brunneoporus Audet, Mushrooms nomenclatural novelties 2: 1, 2017.

Type species - Trametes malicola Berk. \& M.A. Curtis, J. Acad. nat. Sci. Philad., N.S. 3: 209, 1856.

Remarks - According to Audet (2017b), Trametes malicola differs from Antrodia s.str. by deeper brownish basidiomes, distinctly brownish interwoven skeletal hyphae, short basidia, very rarely pedunculate, mostly about 20-25 $\mu \mathrm{m}$ long, and uninucleate, not regularly fusiform distal end basidiospores. Brunneoporus is not related to Antrodia s.str. but constitutes its own lineage within the large Fomitopsis - Daedalea clade (Spirin et al. 2016).

Bulbillomyces Jülich, Persoonia 8: 69, 1974.

Type species - Kneiffia farinosa Bres., Annls mycol. 1(2): 105, 1903.

Basidiome resupinate, effused, hymenophore smooth or pilose by the projecting cystidia. Hyphal system monomitic, hyphae with clamps, more or less cyanophilous. Cystidia thick-walled and encrusted. Basidia urniform or subcylindrical, with 4-sterigmata and a basal clamp. Basidiospores ellipsoid to ovoid, somewhat thick-walled, inamyloid. Sclerotial stage consisting in small white globules. 
Remarks - Bulbillomyces is characterized by the aegeritoid sclerotial stage (Aegerita) formed by small globules, and by the presence in the teleomorphic state of encrusted cystidia and thickwalled basidiospores. Phylogenetically it is classified in the Meruliaceae P. Karst. (Larsson 2007b).

Butlerelfia Weresub \& Illman, Can. J. Bot. 58(2): 144, 1980.

Type species - Butlerelfia eustacei Weresub \& Illman, Can. J. Bot. 58(2): 145, 1980 (=Corticium centrifugum (Lév.) Bresadola, a name based on an anamorph, associated teleomorph: Athelia epiphylla Pers.)

Remarks - A synonym of Athelia Pers. Butlerelfia eustacei is connected with Athelia. The species differs by having constant clamps at the septa, narrower hyphae and no sclerotia, and the production of a basidiome in culture. This species is known from Europe and North America from stored apples and pears, causing fisheye rot.

Butyrea Miettinen, Ann. Bot. Fennici 53: 161, 2016.

Type species - Physisporus luteoalbus P. Karst., Rev. Mycol. (Toulouse) 9: 10, 1887.

Basidiome resupinate, hymenophore poroid with small pores (ab. 4-8 per mm), yellowish. Hyphal system dimitic, hyphae with clamps, skeletal hyphae moderately cyanophilous, narrow and sinuous, mostly $<2.5 \mu \mathrm{m}$ in width, with a distinct lumen. Cystidia of two types: 1) Gloeocystidia, thin-walled, present in hymenium and in tube bottoms, 2) Encrusted tramal cystidia, thick-walled, club-shaped, encrusted part ab. 25-50 x 10-18 $\mu \mathrm{m}$. Basidiospores cylindrical, straight, ab. 4-5 x 1.7-2.3 $\mu \mathrm{m}$, smooth, thin-walled, inamyloid, indextrinoid.

Remarks - Butyrea is closely related to Antrodiella but differing in the presence of gloeocystidia and skeletocystida. Also, it shows some similarities with poroid species of Steccherinum, but in the latter gloeocystidia are lacking (Miettinen \& Ryvarden 2016).

Byssocorticium Bondartsev \& Singer, in Singer, Mycologia 36: 69, 1944.

Type species - Thelephora atrovirens Fr., Elench. fung. (Greifswald) 1: 202, 1828.

Basidiome byssoid, hymenophore smooth or poroid, bluish-green. Hyphal system monomitic, hyphae simple-septate and with few scattered clamps, branched at right angles. Cystidia absent. Basidia clavate, with 4-sterigmata, with numerous granular contents, and with or without basal clamp. Basidiospores globose or subglobose, smooth, distinctly thick-walled, inamyloid, indextrinoid, stained in cotton blue.

Remarks - Byssocorticium is characterized by the byssoid Basidiome with usually bluish colours and by the thick-walled basidiospores. It is close to Piloderma but in this genus lacks the bluish colours and the hyphae are completely simple-septate. According to molecular data, Byssocorticium belongs to the Atheliaceae (Larsson 2007b).

Byssocristella M.P. Christ. \& J.E.B. Larsen, Friesia 9: 313, 1970.

Type species - Byssocristella pallidocitrina M.P. Christ. \& J.E.B. Larsen, Friesia 9: 313, 1970.

Remarks - A synonym of Tomentellopsis Hjortstam.

Byssomerulius Parmasto, Eesti NSV Tead. Akad. Toim., Biol. seer16: 383, 1967.

Type species - Thelephora corium Pers., Syn. meth. fung. (Göttingen) 2: 574, 1801.

Synonym - Ceraceomerulius (Parmasto) J. Erikss. \& Ryvarden.

Basidiome resupinate to pileate, hymenophore smooth to merulioid, whitish to yellowish or brownish, margin white. Hyphal system monomitic, thin to thick-walled, all hyphae without clamps. Cystidia absent or present. Basidia narrowly clavate, with 4-sterigmata, and without a basal clamp. Basidiospores subcylindrical, smooth, inamyloid, indextrinoid.

Remarks - Parmasto (1967) described Byssomerulius to include some species with simpleseptate generative hyphae and merulioid hymenophore with Thelephora corium as the generic type species; Meruliopsis Bondartsev, was described to include Xylomyzon taxicola Pers., with a typical 
poroid hymenophore (Parmasto 1959). Byssomerulius, Candelabrochaete, Ceriporia, Gloeoporus, and Meruliopsis, all sharing generative simple-septate hyphae, are classified by molecular data in the Byssomerulius family, with other clamped species as Phlebia nitidula (P. Karst.) Ryvarden and Ceraceomyces serpens (Tode) Ginns (Larsson 2007b).

Byssoporia M.J. Larsen \& Zak, Can. J. Bot. 56: 1123, 1978.

Type species - Boletus terrestris DC., in De Candolle \& Lamarck, Fl. franç., Edn 3 (Paris) 5/6: 39, 1815.

Basidiome resupinate, effused, soft, hymenophore poroid, margin rhizomorphic with yellowish hyphal strands. Hyphal system monomitic, hyphae with simple-septa or infrequent clamps, thin-walled. Cystidia absent. Basidia clavate, with 4-sterigmata, simple septate at the base. Basidiospores subglobose, smooth, thick-walled, IKI-.

Remarks - Boletus terrestris was originally included in Byssocorticium but later segregated because of the poroid hymenophore, non-blue basidiome, and the clampless basidial bases (Larsen \& Zak 1978). Phylogenetically is classified in the Russulaceae close to the stipitate and pileate poroid genus Albatrellus Gray (Bruns et al. 1998, Larsson 2007b).

Cabalodontia Piątek, Polish Botanical Journal 49(1): 2, 2004.

Type species - Odontia queletii Bourdot \& Galzin, Bull. Soc. mycol. Fr. 30(3): 270, 1914.

Basidiome resupinate, ceraceous to subgelatinous, odontoid, tuberculate or smooth. Hyphal system monomitic, hyphae with clamps. Cystidia lacking or present. Basidia narrowly clavate. Basidiospores non-amyloid, allantoid or ellipsoidal. Nuclear behaviour normal.

Remarks - Based strictly in morphology it could be considered a synonym of Phlebia or Steccherinum. Odontia queletii resembles a species of Steccherinum where it has also been included (Hallenberg \& Hjortstam 1988) sharing a similar micromorphology and a normal nuclear behaviour (astatocoenocytic or exceptionally heterocytic or holocoenocytic in most of Phlebia s.str.), but it differs in the monomitic hyphal system even if the limits between the monomiticdimitic character within Steccherinum species are relative. Eriksson et al. (1981) suggested also the possibility to include Odontia queletii among Mycoacia, a genus including those Phlebia with an odontioid hymenophore. In the molecular analysis by Parmasto \& Hallenberg (2000), Odontia queletii form with Phlebia bresadolae a separate clade from the core of Phlebia s.str. (which include the generic type of Phlebia). Odontia queletii has an odontioid hymenophore and numerous encrusted cystidia, while Phlebia bresadolae Parmasto has a tuberculate hymenophore and lacks cystidia, even variation in hymenophore morphology and presence of cystidial elements is a variable feature within Phlebia s.str. Piątek (2004), based on the normal nuclear behaviour and molecular data generated by Parmasto \& Hallenberg (2000), proposed Cabalodontia to include Odontia queletii and four more species from the Northern Hemisphere.

Caerulicium Jülich, Biblthca Mycol. 85: 395, 1982. 1970.

Type species - Byssocorticium neomexicanum Gilb. \& Budington, Mycologia 62(4): 673,

Remarks - Similar and possibly a synonym of Byssocorticium Bondartsev \& Singer, differing in having globose to broadly ellipsoid, thick-walled basidiospores.

Caldesiella Sacc., Michelia 1(1): 6, 1877.

Type species - Caldesiella italica Sacc., Michelia 1(1): 7, 1877.

Remarks - A synonym of Tomentella (Pers.) Pat.

Campbellia Cooke \& Massee, in Cooke, Grevillea 18: 87, 1890.

Type species - Merulius infundibuliformis Cooke \& Massee, Grevillea 16: 73, 1888.

Remarks - A homonym of Campbellia Wight 1850 (Orobanchaceae) and a synonym of Gyrodon Opat. 
Campylomyces Nakasone, Sydowia 56(2): 261, 2004.

Type species - Aleurodiscus tabacinus Cooke, Grevillea 14(69): 11, 1885.

Basidiome perennial or annual, gregarious, campanulate to cupulate, margin involute, ceraceous or corneous when dried but expanded, elastic, and subgelatinous when hydrated. Hymenophore with numerous sterile hyphal pegs not reacting to potassium hydroxide. Hyphal system monomitic, hyphae with clamps. Cystidia (pseudocystidia) present, yellowish to dark brown, encrusted. Basidia large, clavate, with a stalked base, with 4-sterigmata, and with a basal clamp connection. Basidiospores large, cylindrical, with a distinct, blunt apiculus, thin-walled, hyaline, smooth, inamyloid.

Remarks - Campylomyces is characterized by small, thin, gregarious, sessile, cupulate Basidiome with free involute margins and a ceraceous or corneous texture (Nakasone 2004). It seems to be related to Veluticeps but differs in the absence of colour change in $\mathrm{KOH}$ and basidiome structure.

Candelabrochaete Boidin, Cahiers de La Maboké 8: 24, 1970.

Type species - Candelabrochaete africana Boidin, Cahiers de La Maboké 8(1): 24, 1970.

Basidiome resupinate, effused, byssoid to submembranaceous. Hymenophore smooth to odontioid. Hyphal system monomitic, hyphae thin- to thick-walled, branching at wide angles, smooth, simple-septate, mostly short-celled. Cystidia cylindrical, often with secondary simple septa, thick-walled. Basidia subcylindrical, with 4-sterigmata, clampless at the base. Basidiospores ellipsoid to allantoid, smooth, thin-walled, non-amyloid.

Remarks - Candelabrochaete is characterized by the wide short-celled simple-septate hyphae and the multiseptate cystidia. Some molecular studies seem to indicate that Candelabrochate is polyphyletic (Binder et al. 2005, Larsson 2007b).

Canopora Niemelä, Suomen käävät, Norrlinia 31: 109, 2016.

Type species - not indicated.

Remarks - Niemelä proposed the generic name Canopora to classify Canopora subfuscoflavida (Rotsk) Miettinen \& Spirin (Niemelä 2016: 109, 428) but this generic name is not validly published (Art. 40.1, Shenzhen code). According to Spirin (pers. comm.), the type of Polyporus lindbladii (from South Carolina, FH) belongs to Trichaptum sector (Ehrenb.) Kreisel so, it is necessary to introduce a new genus name for Polyporus subfuscoflavidus Rostk. To avoid nomenclatural confusion, we are not introducing here the name Canopora until it will be formally published.

Caripia O. Kuntze, Rev. Gen. Pl. 3: 451, 1898.

Type species - Hypolyssus montagnei Berk. London J. Bot. 1(3): 139, 1842.

Basidiome small, up to $1 \mathrm{~cm}$, gregarious, centrally stipitate, obconical to turbinate with a flat and more or less discoid pileus, tomentose, whitish, hymenial surface plicate to sublamellate. Context cartilaginous when dry, hard when dry. Hyphal system monomitic, generative hyphae with clamps, hyaline, branched or not, thin- to thick-walled. Cystidia hyaline, fusiform, embedded in the hymenial layer. Basidia clavate to cylindrical, 2- to 4-spored, Basidiospores ellipsoid to navicular, smooth, hyaline, non-amyloid.

Remarks - Caripia is a tropical genus distributed from Northern Argentina to Southern Mexico (Kuntze 1898). Molecular analyses showed that it is a reduced form of the agaric Gymnopus (Pers.) Roussel.

Cartilosoma Kotl. \& Pouzar, Česká Mykol. 12(2): 101, 103, 1958.

Type species - Trametes subsinuosa Bres., Annls mycol. 1(1): 82, 1903 (=Polyporus ramentaceus Berk. \& Broome, Ann. Mag. nat. Hist., Ser. 5 3: 210, 1879).

Basidiome annual, resupinate, normally small and rounded, tough when fresh, with a cartilaginous consistency, hard when dry, margin narrow, white and floccose; pore surface white, 
becoming buff to straw-colored or unevenly resinous brown, pores angular; taste bitter. Hyphal system dimitic, generative hyphae abundant, hyaline, with clamps, thin to thick-walled, in parts slightly gelatinized in $\mathrm{KOH}$, moderately to frequently branched, skeletal hyphae solid to thickwalled, straight, non-septate, common in the subiculum, scattered in the trama. Cystidia absent, but fusoid, hyaline. thin-walled cystidioles common, especially in immature hymenia. Basidia clavate, 4-sterigmate, with a basal clamp. Basidiospores cylindric to narrowly ellipsoid, hyaline, thinwalled, smooth, IKI-. Causing a brown rot.

Remarks - Cartilosoma is a genus segregated from Antrodia on the basis of the cartilaginous consistency of the basidiome (Pouzar 1958). The generic type C. ramentaceum has a quite isolated position among the Antrodia species, but further phylogenetical analyses are needed to establish is position. See also Bernicchia \& Gorjón (2020).

Castanoporus Ryvarden, Synopsis Fung. 5: 121, 1991.

Type species - Merulius castaneus Lloyd, Lloyd Mycol. Writ. 4: 555, 1916.

Basidiome annual, resupinate, effused, margin wide, cinnamon, hymenial surface poroid, pores angular to irpicoid, cinnamon to purplish, 1-2 per mm. Hyphal system monomitic, generative hyphae simple-septate, thin- to thick-walled, with reddish to brown granular encrustation. Cystidia conical, thick-walled, encrusted in the apical part. Basidia clavate, with 4-sterigmata, and a simple basal septum. Basidiospores cylindrical to broadly allantoid, smooth, thin-walled, hyaline, IKI-.

Remarks - Castanoporus is characterized by the cinnamon, irpicoid resupinate basidiomes, microscopically the simple-septate hyphae, encrusted cystidia and cylindrical basidiospores are diagnostic (Ryvarden 1991). Oxyporus is quite similar, differing above all in the globose basidiospores and light coloured basidiomes. Castanoporus is considered traditionally among the polypore fungi but it is included here by the resupinate basidiome and irpicoid basidiome.

Caudicicola Miettinen, M. Kulju \& Kotir., Ann. bot. fenn. 54: 163, 2017.

Type species - Caudicicola gracilis Miettinen, M. Kulju \& Kotir., Ann. bot. fenn. 54: 163, 2017.

Basidiome annual, resupinate, very fragile, pale creamish white or pale yellow when dry, with a very thin white subiculum, pores angular, 3-4 per mm, with thin, partly lacerate dissepiments, old basidiomes have sometimes pulverulent areas at margin, which contain masses of conidia, but no rhizomorphs. Hyphal system monomitic, hyphae relatively thin-walled, CB-, IKI-, subiculum consists of narrow hyphal strands and loosely arranged randomly orientated hyphae mostly with normal clamps, but ampullaceous septa fairly common; conidiogenous hyphae double walled. Cystidia and cystidioles absent. Basidia subcylindrical, with (2)-4 sterigmata, which are exceptionally long, typically $5 \mu \mathrm{m}$ but even up to $8 \mu \mathrm{m}$, basally clamped. Basidiospores smooth, subglobose, broadly ellipsoid or pip-shaped, fairly thin-walled, with a relatively large apiculus, commonly with one or many shiny, irregular bodies inside, CB-, IKI-. Conidia ellipsoid, often broadly fusiform, reminding a protozoan ciliate, slightly thick-walled, finely ornamented or more rarely smooth, $\mathrm{CB}+$, IKI-. Probably causing a white rot.

Remarks - Caudicicola is characterized by very fragile basidiomes, monomitic hyphal structure with clamps, short and wide tramal cells, smooth ellipsoid spores, basidia with long sterigmata and conidiogenous areas in the margins of the basidiome producing verrucose, slightly thick-walled conidia. The genus belongs to the residual polyporoid clade of the Polyporales in the vicinity of Steccherinaceae but has no known close relatives (Kotiranta et al. 2017).

Cejpomyces Svrček \& Pouzar, Česká Mykol. 24: 5, 1970.

Type species - Corticium terrigenum Bres., Annls mycol. 1(2): 98, 1903.

Remarks - A synonym of Thanatephorus Donk.

Ceraceohydnum Jülich, Persoonia 10(1): 138, 1978.

Type species - Ceraceohydnum brunneum Jülich, Persoonia 10(1): 138, 1978. 
Basidiome annual, resupinate, effused, ceraceous, margin indistinct, hyphal strands lacking, hymenophore hydnoid, with long aculei, brownish. Hyphal system dimitic, skeletal hyphae hyaline, thick-walled, generative hyphae hyaline, thick-walled, with clamps. Basidia narrowly clavate, small, with 4-sterigmata, and a basal clamp. Basidiospores ellipsoid to subglobose, small, hyaline, smooth, inamyloid.

Remarks - It was considered a synonym of Mycoaciella by Hjortstam et al. (1990) but it was accepted as a separated genus by Nakasone (2002) because of its small basidia and basidiospores, and absence of cystidia.

Ceraceomerulius (Parmasto) J. Erikss. \& Ryvarden, Cortic. N. Eur. (Oslo) 2: 196, 1973.

Basionym - Byssomerulius subgen. Ceraceomerulius Parmasto, Consp. System. Corticiac. (Tartu): 80, 1968.

Type species - Merulius rubicundus Litsch., Bull. trimest. Soc. mycol. Fr. 49: 293, 1934.

Remarks - A synonym of Byssomerulius Parmasto.

Ceraceomyces Jülich, Willdenowia Beih. 7: 146, 1972.

Synonym - Athelia subsect. Tessulatae Parmasto.

Type species - Corticium tessulatum Cooke, Grevillea 6(40): 132, 1878.

Basidiome resupinate, at first pellicular, thickening in age and with a ceraceous consistency, separable from the substrate, hymenophore smooth to merulioid. Hyphal system monomitic, hyphae thin-walled, with clamps, subicular hyphae loosely interwoven, more densely arranged in the subhymenium. Cystidia present or absent. Basidia narrowly clavate, in a dense palisade, (2)4sterigmata, with basal clamp. Basidiospores subglobose to narrowly ellipsoid, smooth, guttulate, thin-walled, IKI-.

Remarks - Ceraceomyces seems to derive from Athelia that has short lived basidiome and a restricted number of basidia are produced in open branched clusters, in a loose hymenial structure (Jülich 1972). On the contrary, in Ceraceomyces the basidia are produced in prolonged series generating a dense and closely packed palisade of narrow, clavate basidia. Phylogenetic relationships in Ceraceomyces are still unclear, it seems to be polyphyletic, while Ceraceomyces tessulatus (Cooke) Jülich, the generic type species, and Ceraceomyces borealis (Romell) J. Erikss. \& Ryvarden, cluster in the Amylocorticiaceae with Amylocorticiellum subillaqueatum and Amylocorticium subincarnatum. Other Ceraceomyces species cluster within clampless genera such as Byssomerulius, Ceriporia, Phanerochaete, or Phlebiopsis (Larsson 2007b).

Ceraceopsis Hjortstam \& Ryvarden, Synopsis Fungorum 23: 62, 2007.

Type species - Ceraceopsis verruculosa Hjortstam \& Ryvarden, Synopsis Fungorum 23: 64, 2007.

Basidiome resupinate, effuse, pellicular to membranous, hymenophore smooth, subiculum distinct, whitish to pale yellowish, margin fimbriate, rhizomorphs rare or absent. Hyphal system monomitic, hyphae straight, distinct, simple-septate, thin-walled or slightly thickened, hyaline, or pale yellowish brown in the subiculum. Cystidia absent. Basidia almost clavate, with 4-sterigmata, and a simple basal septum. Basidiospores subglobose to ellipsoid, finely rugose, thin-walled or with slightly thickened walls, hyaline, inamyloid, indextrinoid, cyanophilous.

Remarks - Ceraceopsis is characterized by the smooth, pellicular to membranaceous basidiome, simple-septate hyphae, and warted and cyanophilous basidiospores (Hjortstam \& Ryvarden 2007). Coniophoropsis has also simple-septate hyphae and cyanophilous verrucose but large basidiospores, the basidioma is brown coloured with an olivaceous tint, and basidiospores are distinctly thick-walled and yellowish-brown.

Cerarioporia F. Wu, L.W. Zhou \& Jing Si, Phytotaxa 280(1): 57, 2016. 2016.

Type species - Cerarioporia cystidiata F. Wu, L.W. Zhou \& Jing Si, Phytotaxa 280(1): 58, 
Basidiome annual, resupinate, waxy to resinous. Pore surface white to cream when fresh, dirty cinnamon when dry. Subiculum cinnamon buff, resinous, very thin to almost lacking. Tube layer concolorous with the pore surface. Hyphal system dimitic; generative hyphae bearing clamp connections; skeletal hyphae dominant; presence of thick-walled and encrusted cystidia. Basidiospores hyaline, thin-walled, smooth, fusiform, IKI-, CB-. Causing a white rot.

Remarks - Cerarioporia is characterized by a resupinate basidiome with large pores, waxy to resinous tubes, a dimitic hyphal structure with clamp connections, distinctly thick-walled and encrusted cystidia, hyaline, thin-walled, smooth and fusiform basidiospores, all structures negative in both Cotton Blue and Melzer's reagent. Phylogenetic analyses show that Cerarioporia belongs to the core polyporoid clade of Polyporales (Wu et al. 2016).

Ceratobasidium D.P. Rogers, Univ. Iowa Stud. nat. Hist. 17: 4, 1935.

Type species - Ceratobasidium calosporum D.P. Rogers, Univ. Iowa Stud. nat. Hist. 17(1): 5, 1935.

Basidiome resupinate, very thin, ceraceous, hymenophore smooth, greyish. Hyphal system monomitic, hyphae without clamps, basal hyphae more or less thick-walled. Cystidia absent. Basidia subglobose, with 2-4 long sterigmata, without a basal clamp. Basidiospores ellipsoid to fusiform, smooth, thin-walled, repetitive.

Remarks - Ceratobasidium is mainly characterized by the repetitive basidiospores, the simple-septate hyphae, and the almost globose basidia (Rogers 1935). It is very close to Thanatephorus and Botryobasidium. The latter genus is distinguished by the absence of spore repetition, and Thanatephorus differs in the basidial and hymenial morphology and structure. In some molecular studies, Binder et al. (2005), Moncalvo et al. (2006) Ceratobasidium is included in the cantharelloid clade forming a monophyletic group with Thanatephorus and very closely related to Botryobasidium.

Cericium Hjortstam, Mycotaxon 54: 184, 1995.

Type species - Amethicium luteoincrustatum Hjortstam \& Ryvarden, Mycotaxon 25: 542, 1986.

Basidiome resupinate, thick, stratified, fairly brittle and ceraceous, hymenophore smooth or slightly tuberculate, pale coloured or ochraceous. Hyphal system dimitic, subicular hyphae arboriform (binding hyphae), hyaline, without clamps, generative hyphae thin-walled, hyaline to yellowish, with clamps. Cystidia thin-walled, basally strongly encrusted with resinous yellowish matter, about 70-100 $\mu \mathrm{m}$ long. Basidia subclavate, in a rather dense layer, with 4-sterigmata, and a basal clamp. Basidiospores ellipsoid, smooth, thin-walled, IKI-, CB-.

Remarks - Cericium is characterized by the binding hyphae and numerous cystidia with resinous encrustation. Amethicium is superficially similar but is distinct in its striking violet colour and lack of cystidia (Hjortstam 1995).

Cerinomyces G.W. Martin, Mycologia 41: 82, 1949.

Type species - Cerinomyces pallidus G.W. Martin, Mycologia 41(1): 83, 1949.

Basidiome resupinate, hymenophore smooth to tuberculate, ceraceous when fresh, brittle when dry. Hyphal system monomitic, hyphae with clamps, thin- to slightly thick-walled. Cystidia absent. Basidia clavate, with two very long sterigmata. Basidiospores ellipsoid to allantoid, smooth, thin-walled, non-amyloid.

Remarks - Cerinomyces is characterized by its ceraceous basidiome and basidia with two long sterigmata that could indicate a possible relationship with species in Dacrymycetales (Martin 1949).

Cerioporus Quél., Enchir. fung. (Paris): 167, 1886.

Type species - Boletus squamosus Huds., Fl. Angl., Edn 2: 626, 1778. 
Remarks - According to molecular data by Justo \& Hibbett (2011) and Zmitrovich \& Kovalenko (2016), Boletus squamosus forms with a distinct clade of resupinate to stipitate polypore species, named 'Datronia clade', where the genus Cerioporus was reintroduced by Zmitrovich \& Kovalenko (2016).

Ceriporia Donk, Med. Bot. Mus. Univ. Utrecht. 9: 170, 1933.

Type species - Polyporus viridans Berk. \& Broome., Ann. Mag. Nat. Hist. 3(7): 379, 1861.

Basidiome annual, resupinate, hymenial surface poroid with ceraceous pores, fragile when dry, variable in colour, whitish, cream, pinkish, greenish to purplish. Hyphal system monomitic, generative hyphae simple-septate, usually characteristically and conspicuously encrusted. Cystidia absent. Basidia clavate, with 4-sterigmate, and a simple basal septum. Basidiospores variable in shape, allantoid to ellipsoid or subglobose, smooth, thin-walled, hyaline, IKI-. On dead coniferous and deciduous wood, causing a white rot.

Remarks - Ceriporia is usually easy to distinguish by the poroid ceraceous hymenophore, simple-septate hyphae usually encrusted, and hyaline, smooth basidiospores, not reacting in Melzer's reagent. See also Ryvarden \& Melo (2017) and Bernicchia \& Gorjón (2020).

Ceriporiopsis Domański, Acta Soc. Bot. Pol. 32: 731, 1963.

Type species - Poria gilvescens Bres., Ann. Mycol. 6: 40, 1908.

Basidiome annual, resupinate, pores round to angular, hymenophore usually light coloured, margin sometimes rhizomorphic. Hyphal system monomitic, generative hyphae with clamps. Cystidia absent, cystidiols may be present. Basidia clavate, with 4-sterigmata, and a basal clamp. Basidiospores cylindrical to globose, smooth, thin-walled, hyaline, IKI-. On deciduous and coniferous wood, causing a white rot.

Remarks - Ceriporiopsis is characterized by the monomitic hyphal system with clamped generative hyphae, smooth spores not reacting in Melzer's reagent, and the type of white rot. Morphologically, Ceriporiopsis comes in some respects close to Oligoporus-Postia that differs in the brown rot type. The genus is polyphyletic as shown in several studies (Tomšovský et al. 2010). Some species have transferred to Niemelaea, Gelatoporia, and Pouzaroporia.

Cerocorticium Henn., Monsunia 1: 138, 1900.

Type species - Cerocorticium bogoriense Henn. \& E. Nyman, Monsunia 1: 138, 1900. (=Corticium molle Berk. \& M.A. Curtis, J. Linn. Soc., Bot. 10(46): 336, 1868).

Basidiome resupinate, effused, ceraceous, hymenial surface more or less smooth, yellow to orange. Hyphal system monomitic, hyphae hyaline, thin- or thick-walled, with clamps. Paraphysoid hyphae present. Cystidia absent. Basidia large, clavate or broadly cylindrical, with a basal clamp. Basidiospores hyaline, smooth, large, more or less thin-walled, inamyloid, with a rather large apiculus.

Remarks - Cerocorticium is mainly distinguished by the yellow to the orange hymenial surface and large narrowly ellipsoid to subcylindrical thin-walled basidiospores. Monotypic genus.

Chaetoderma Parmasto, Consp. System. Corticiac. (Tartu): 86, 1968.

Type species - Peniophora luna Romell ex D.P. Rogers \& H.S. Jacks., Farlowia 1(2): 320, 1943.

Remarks - A later homonym of Chaetoderma Kütz. 1843 (Algae). Chaetodermella S. Rauchster was proposed to replace Chaetoderma Parmasto.

Chaetodermella Rauschert, Haussknechtia 4: 52, 1988.

Type species - Peniophora luna Romell ex D.P. Rogers \& H.S. Jacks., Farlowia 1(2): 320, 1943.

Basidiome resupinate, thick, hymenophore smooth, whitish to greyish. Hyphal system monomitic, hyphae with clamps. Cystidia tubular, thick-walled, encrusted. Basidia long tubular, 
with 4-sterigmata, and with a basal clamp. Basidiospores fusoid, smooth, thin-walled, inamyloid, indextrinoid.

Remarks - Chaetodermella is a well-characterized genus by the thick-walled cystidia and the fusoid basidiospores. Phylogenetically its relationships are unclear (Larsson 2007b).

Chaetoporellus Bondartsev \& Singer, in Singer, Mycologia 36: 66, 1944.

Type species - Poria latitans Bourdot \& Galzin, Bull. trimest. Soc. mycol. Fr. 41: 226, 1925.

Basidiome resupinate, effused, hymenophore poroid or hydnoid. Hyphal system monomitic, all hyphae with clamps, thin- to thick-walled. Cystidia (leptocystidia) cylindrical, thin or with distinct walls. Basidia clavate, with 4-sterigmata and a basal clamp. Basidiospores allantoid, smooth, thin-walled, hyaline, IKI-.

Remarks - Chaetoporellus is micromorphologically closely related to Kneiffiella, and mainly separated by the typical poroid hymenophore, thin-walled leptocystidia and small allantoid basidiospores. Phylogenetically, it seems to be related to Alutaceodontia and Kneiffiella in the Hymenochaetales, (Larsson et al. 2006, Larsson 2007b) and differing mainly by the poroid hymenophore. Chaetoporellus is treated as a synonym of Kneiffiella P. Karst. by Riebesehl \& Langer (2017).

Chondrostereum Pouzar, Česká Mykol. 13: 17, 1959.

Type species - Stereum purpureum Pers., Neues Mag. Bot. 1: 110, 1794.

Basidiome usually effuse-reflexed (stereoid), flexible, composed of several layers in section, upper sterile surface more or less tomentose, hymenophore smooth. Hyphal system monomitic, hyphae with clamps. Cystidia (leptocystidia) present. Basidia tubular, with 4-sterigmata, with a basal clamp. Basidiospores cylindrical, smooth, thin-walled, IKI-.

Remarks - Chondrostereum is macroscopically similar to Stereum differing mainly in the pinkish to violaceous colour, but microscopically they are not related; basidiospores are inamyloid and typical leptocystidia are present in Chondrosterum (Pouzar 1959). Molecular studies by Larsson et al. (2004) show that C. purpureum is closely related to Cylindrobasidium laeve in the euagarics clade and separated from the rest of corticioid fungi.

Christiansenia Hauerslev, Friesia 9(1-2): 43, 1969.

Type species - Christiansenia pallida Hauerslev, Friesia 9(1-2): 43, 1969.

Remarks - A synonym of Syzygospora G.W. Martin. See comments under this genus.

Chrysoderma Boidin \& Gilles, Cryptog. Mycol. 12(2): 126, 1991.

Type species - Chrysoderma alboluteum Boidin \& Gilles, Cryptog. Mycol. 12(2): 127, 1991.

Remarks - A synonym of Cerocorticium Henn. Hjortstam \& Ryvarden (2007) indicated the similarity with Cerocorticium molle, and the synonym has recently been confirmed by Nakasone (2015).

Cinereomyces Jülich, Bibliotheca Mycologica 85: 396, 1982.

Type species - Polyporus lindbladii Berk., Grevillea 1 (4): 54, 1872.

Basidiome annual, resupinate, becoming widely effused, soft to tough, margin white, narrow to wide. Pore surface white to grayish, pores circular, context white and cottony, azonate. Hyphal system di- to trimitic, generative hyphae with clamps, hyaline. thin-walled, skeletal hyphae straight to sinuous, thick-walled to solid, non-septate, rarely branched, gelatinizing and disappearing in $\mathrm{KOH}$, weakly amyloid in Melzer's reagent, most easily seen in hyphal masses, binding hyphae narrow and richly branched, observed only in the context, apparently rare. Cystidia none, but fusoid, non-projecting cystidiols occur scattered among the basidia. Basidia clavate, 4-sterigmate, with a basal clamp. Basidiospores allantoid to cylindrical, hyaline, thin-walled, negative in Melzer's reagent. Causing a white rot. 
Remarks - Cinereomyces, is characterized by the dimitic to trimitic hyphal system with weakly amyloid vegetative hyphae, and allantoid to cylindrical basidiospores, non-reacting in Melzer's reagent. Cinereomyces lindbladii (Berk.) Jülich, the generic type, has also been usually classified under Diplomitoporus but we want to highlight the amyloidity of the vegetative hyphae that has been proved to be a useful character among other genera of polypores. It is separated from Amyloporia by the type of decay, brown rot in the latter. According to Miettinen \& Rajchenberg (2012), the 'Cinereomyces clade' comprises the genera Sebipora, Gelatoporia, Cinereomyces and Obba. The 'Cinereomyces clade' is well-delimited in nrDNA-based phylogenetic analysis, but its position in Polyporales remains unclear. Its closest relative may be found in the core polyporoid clade. Only a few morphological characters are common for all the species in the clade, e.g. CBand CRB+ hyphae, white fruiting bodies, presence of oil, and middle-sized spores. Niemelä (2016) proposed the generic name Canopora to classify Polyporus subfuscoflavidus (=Polyporus lindbladii) but this generic name is not validly published (Art. 40.6, Shenzhen code).

Cinereomycetella Zmitr., Folia Cryptogamica Petropolitana 6: 93, 2018.

Type species - Poria overholtsii Pilát, Stud. Bot. Čechoslov. 3: 2, 1940.

Basidiome of fibroporioid habitus, annual, widely effused, hymenophore a single tube layer, soft. Subiculum byssoid, white. Hyphal system monomitic in tubes and moderately dimitic in subiculum. Generative hyphae with clamp connections, hyaline, CB-. Leptocystidia fusoid, thinwalled. Basidia clavate, with a central constriction, 4-spored, clamped at the base. Basidiospores ellipsoid, thin-walled, IKI-, CB-. Causing a white rot.

Remarks - Cinereomycetella is similar to Cinereomyces but differs by monomitic tubes and ellipsoid (vs. cylindrical) basidiospores (Zmitrovich 2018). Poria overholtsii Pilát is a North American species and was treated under Diplomitoporus by Gilbertson \& Ryvarden (1986).

Cladoderris Pers. ex Berk., J. Bot., Lond. 1(3): 152, 1842.

Type species - Thelephora dendritica Pers., in Gaudichaud-Beaupré in Freycinet, Voy. Uranie. Bot. 5: 176, 1827.

Remarks - A synonym of Cymatoderma Jungh.

Clavulicium Boidin, Bull. Soc. Hist. nat. Toulouse 92: 280, 1957.

Type species - Corticium pilatii Boidin [as 'pilat'], Bull. mens. Soc. linn. Lyon 8: 231, 1954.

Basidiome resupinate, subceraceous to membranaceous, hymenophore smooth. Hyphal system monomitic, hyphae with clamps, thin-walled. Gloeocystidia present. Basidia clavate, 2spored, with a basal clamp, guttulate. Basidiospores ellipsoid, smooth, thin-walled, inamyloid, indextrinoid, distinctly guttulate.

Remarks - Clavulicium is characterized by the clamped generative hyphae, the presence of gloeocystidia and the large ellipsoid basidiospores, with many guttulate contents. Corticium delectabile Jacks., and Corticium spurium Bourdot have usually been classified in Clavulicium, but they are presently included in Membranomyces. They differ above all in the simple-septate hyphae and in different basidial morphology. The relationships of Clavulicium with other corticioid genera seems still unclear (Larsson 2007b).

Climacodon P. Karst., Revue mycol., Toulouse 3(9): 20, 1881.

Type species - Hydnum septentrionale Fr., Syst. mycol. (Lundae) 1: 414, 1821.

Basidiome annual, pileate, imbricate, upper sterile surface fibrillose to scrupose, hymenophore hydnoid, with long aculei. Hyphal system monomitic, generative hyphae with simple septa, but some septa may have clamp connections. Cystidia present, as gloeocystidia or thickwalled cystidia. Basidia clavate, with 4-sterigmata and with a simple basal septum. Basidiospores ellipsoid, smooth, thin-walled, IKI-.

Remarks - Climacodon is easily recognized by the large pileate basidiome with hydnoid hymenophore and imbricate growth. It reminds Hericium in some macroscopical aspects, but the 
latter has amyloid basidiospores, and is phylogenetically classified in the Russulales, whereas Climacodon in the Polyporales (Larsson 2007b). It is also morphologically close to Mycorrhaphium which has skeletal hyphae in the aculei and context, and to Mycoleptodonoides with a monomitic hyphal system and lacking gloeocystidia. Contrary, Mycorrhaphium and Mycoleptodonoides have clamped basidia.

Colospora Miettinen \& Spirin, Fungal Diversity 75: 232, 2015.

Type species - Colospora andalasii Miettinen \& Spirin, Fungal Diversity 75: 232, 2015.

Basidiome resupinate, minutely odontioid (with sterile hyphal pegs), corticioid, on dead wood. Hyphal system dimitic throughout, clamps present, hyphae short-branched, skeletal hyphae dominating in all parts of the basidiome. Dendrohyphidia common, aculei sterile towards the tip. Basidia utriform, with four sterigmata and a basal clamp. Basidiospores large, thin-walled, finely ornamented (in the type species) or smooth, biapiculate, apical parts distinctly tapering and refractive, with numerous oils drops inside, faintly cyanophilous, showing small amyloid patches in apices.

Remarks - Colospora contains two Epithele-like fungi with a dimitic hyphal structure and biapiculate spores. The type species of Epithele (E. typhae) and Skeletohydnum (S. nikau), the two existing genus names for Epithele-like fungi, are not particularly closely related. Epithele typhae (Pers.) Pat. is a monomitic species with smooth, fusiform spores. Skeletohydnum nikau (G. Cunn.) Jülich also has smooth, fusiform spores, which are thick-walled, and skeletal hyphae are restricted in its aculei making the basidiomes more fragile than in Colospora (see comments by Miettinen \& Spirin in Ariyawansa et al. 2015).

Columnocystis Pouzar, Česká Mykol. 13(1): 17, 1959.

Type species - Thelephora abietina Pers., Syn. meth. fung. (Göttingen) 2: 573, 1801.

Remarks - A synonym of Veluticeps (Cooke) Pat. See Hjortstam \& Telleria (1990).

Columnodontia Jülich, Persoonia 10(3): 326, 1979

Type species - Columnodontia resupinata Jülich, Persoonia 10(3): 327, 1979

Remarks - A synonym of Phlebia Fr. Columnodontia was erected to accommodate resupinate corticioids (similar to species of Phlebia) with a membranaceous-ceraceous to crustaceous basidiome, with a hydnoid hymenophore with small aculei microscopically composed by generative hyphae and projecting columns of crystals.

Confertextum Priyanka \& Dhingra, in Dhingra, Proceedings of the 8th International Conference on Mushroom Biology and Mushroom Products (ICMBMP8) 2014: 25, 2014.

Type species - Confertextum microsporum Priyanka \& Dhingra, Proceedings of the 8th International Conference on Mushroom Biology and Mushroom Products (ICMBMP8) 2014: 27, 2014.

Remarks - Nom. inval., Art. 32.1(a); see Art. 29.1 (Shenzhen code).

Conferticium Hallenb., Mycotaxon 11: 447, 1980.

Type species - Gloeocystidium insidiosum Bourdot \& Galzin, Bull. Soc. mycol. Fr. 28(4): 370, 1913.

Basidiome resupinate, effused, membranaceous to ceraceous, hymenophore smooth to tuberculate. Hyphal system monomitic, hyphae with simple septa, cyanophilous, subhymenial hyphae vertically arranged in a compact and dense structure (pseudoparenchymatic). Cystidia (gloeocystidia) numerous, cylindrical, sinuous, SA+. Basidia clavate, with internal repetition, with 4-sterigmata and a simple basal septum. Basidiospores ellipsoid, smooth or ornamented, thinwalled, amyloid, CB-.

Remarks - Conferticium was separated from Gloeocystidiellum (G. ochraceum group in Eriksson \& Ryvarden 1976) on the basis of the pseudoparenchymatic context, simple-septate and 
cyanophilous hyphae, gloeocystidia SA+ and the internal basidial repetition (Hallenberg 1980). Conferticium belongs to the Stereaceae in the Russulales (Larsson 2007b).

Confertobasidium Jülich, Willdenowia, Beih. 7: 167, 1972.

Type species - Corticium olivaceoalbum Bourdot \& Galzin, Bull. Soc. mycol. Fr. 27(2): 239, 1911.

Remarks - A synonym of Scytinostromella Parmasto.

Coniobotrys Pouzar, Česká Mykol. 12(1): 32, 1958.

Type species - Coniophora ochroleuca Bres., Jber. Westfäl. Prov.-Vereins 26: 130, 1898.

Remarks - A synonym of Jaapia Bres.

Coniophora DC., Fl. franç. (Paris) 5/6: 34, 1815.

Synonym - Coniophorella P. Karst.

Type species - Coniophora membranacea DC., in De Candolle \& Lamarck, Fl. franç., Edn 3 (Paris) 5/6: 34, 1815.

Basidiome resupinate, effused, membranaceous, adnate to separable, hymenophore smooth to tuberculate, usually brown-coloured, margin frequently fibrillose or with hyphal strands. Hyphal system monomitic (di- or trimitic in some species), hyphae generative usually without clamps (some species with clamps at all septa), simple or verticillate clamps sometimes present in the basal hyphae, frequently encrusted. Cystidia absent (present in one species). Basidia cylindrical, with a median constriction, with 4-sterigmata and without a basal clamp. Basidiospores ellipsoid, ovoid or fusiform, smooth, double-walled, with a prominent apiculus, pale yellowish to brownish, cyanophilous, variably dextrinoid.

Remarks - Coniophora is usually easy to distinguish by the brownish smooth to tuberculate Basidiome, and microscopically by the thick-walled and usually strongly dextrinoid basidiospores. Leucogyrophana and Serpula are a sister genus of Coniophora, differing in the typically merulioid to irpicoid hymenophore and the presence of clamps in the generative hyphae. Molecular studies by Binder \& Hibbett (2002) pointed out the evolution of resupinate basidiome deriving from erect ones. Studies by Larsson et al. (2004) and Binder \& Hibbett (2006), show the relationship between Coniophora and other species of the Boletaceae included in the bolete clade (see also Ginns 1982, Hallenberg 1985).

Coniophorafomes Rick, Brotéria, sér. Ci. Nat. 3: 167, 1934.

Type species - Coniophora stereoidea Rick, Brotéria, sér. Ci. Nat. 3: 166, 1934.

Remarks - A synonym of Gloeocystidiellum Donk. See Rick (1934).

Coniophorella P. Karst., Bidr. Känn. Finl. Nat. Folk 48: 438, 1889.

Type species - Hypochnus olivaceus Fr., Observ. mycol. (Havniae) 2: 282, 1818.

Remarks - A synonym of Coniophora DC. Coniophorella was segregated from Coniophora by the presence of encrusted cystidial elements.

Coniophoropsis Hjorstam \& Ryvarden, Mycotaxon 25: 540, 1986.

Type species - Coniophoropsis obscura Hjorstam \& Ryvarden, Mycotaxon 25: 540, 1986.

Basidiome resupinate, effused, closely adnate, hymenial surface smooth, brown violet or with an olivaceous tint, margin paler, whitish, subiculum distinct, loose and soft, whitish. Hyphal system monomitic, hyphae simple-septate, hyaline or with a yellowish tint, thin-walled, smooth or encrusted. Dendrohyphidia poorly differentiated, with few more or less perpendicular branches, protruding above the basidial layer. Cystidia absent. Basidia cylindrical to suburniform, with a median constriction, with 4-sterigmata, with a simple basal septum. Basidiospores subfusiform to subamygdaliform, verrucose, distinctly thick-walled, with yellowish-brown spore walls, inamyloid, indextrinoid, strongly cyanophilous. 
Remarks - Coniophoropsis reminds Coniophora but differs in the indextrinoid and verrucose basidiospores, and in the total absence of clamps in subicular hyphae. In the ITS phylogenetic tree of Boletales, Coniophoropsis bambusicola S.H. He \& Nakasone, and the Coniophora species formed a fully supported clade (Zhao et al. 2018).

Conohypha Jülich, Persoonia 8: 303, 1975.

Type species - Corticium albocremeum Höhn. \& Litsch., Wiesner Festschrift (Wien): 61, 1908.

Basidiome resupinate, effused, adnate, membranaceous, hymenophore smooth, whitish to cream, margin indeterminate. Hyphal system monomitic, hyphae with clamps, short-celled, broad, thin-walled, hyaline. Cystidia absent. Basidia cylindrical, thin-walled, with 4-sterigmata, and a basal clamp. Basidiospores ellipsoid, smooth, thin-walled, hyaline, IKI-.

Remarks - Conohypha is mainly characterized by broad short-celled subhymenial cells. It may remind to Brevicellicium, but it could be easily separated by the larger basidiospores. The phylogenetic relationships of Conohypha with other corticioid fungi remain still unclear (Larsson 2007b).

Coralloderma D.A. Reid, Nova Hedwigia, Beih. 18: 332, 1965.

Type species - Thelephora acroleuca Pat., Bull. Soc. mycol. Fr. 14: 188, 1898.

Basidiome terrestrial, stipitate, coriaceous, spathulate, flabellate to infundibuliform. Pilear surface minutely velutinate to rugose with coralloid hyphae, hymenial surface smooth. Stipe lateral to central, velutinous. Context black. Hyphal system monomitic, generative hyphae usually straight, hyaline to pale brown, thin-walled, clamps present or absent, with purpure brown encrustations in $\mathrm{KOH}$. Cystidia and gloeocystidia absent. Basidia long clavate, 2-4-sterigmate. Basidiospores ellipsoid, thin-walled, hyalinae, inamyloid.

Remarks - It is characterized by the blackish stipitate basidiomes and presence of coralloid hyphae (Reid 1965).

Cordochaete Sanyal, Samita, Dhingra \& Avneet P. Singh, Mycotaxon 123: 103, 2013.

Type species - Cordochaete cystidiata Sanyal, Samita, Dhingra \& Avneet P. Singh, Mycotaxon 123: 105, 2013.

Basidiome resupinate, adnate, effused, hymenial surface corneous as dry. Hyphal system monomitic, generative hyphae without clamps, basal zone very thin, context conspicuous, of loosely interwoven hyphae, subhymenium compact, hyphal cordons both in context and subhymenium. Cystidia thick-walled, encrusted. Basidia clavate, 4-sterigmate, without basal clamp. Basidiospores ellipsoidal to subcylindrical, inamyloid, CB-.

Remarks - Cordochaete, according to the authors, differs from Phlebiopsis in having hyphal cordons and is distinguished from Rhizochaete by the absence of a red or violet $\mathrm{KOH}$ reaction in both the hymenium and hyphal cordons (Sanyal et al. 2013). It is most similar to Phanerochaete differing in the presence of hyphal chords in the subhymenium.

Corneromyces Ginns, Mycologia 68(5): 970, 1976.

Type species - Corneromyces kinabalui Ginns, Mycologia 68(5): 970, 1976.

Basidiome resupinate, effuse, hymenophore smooth to granulose or with sterile hyphal pegs, brown to amber or grayish brown. Hyphal system monomitic, hyphae with clamps, hyaline to brownish, slightly darkening in IKI and blackening in KOH. Dendrophyses present. Cystidia absent. Basidia clavate, with a narrower base, with 4-sterigmate, and a basal clamp. Basidiospores narrowly ellipsoid to cylindrical, smooth, very thick-walled, at first hyaline, becoming brown, cyanophilous, amyloid.

Remarks - Corneromyces is characterized above all by the large, thick-walled, smooth and brown basidiospores that are strongly amyloid and cyanophilous (Ginns 1976). Monotypic genus. 
Coronicium J. Erikss. \& Ryvarden, Cortic. N. Eur. (Oslo) 3: 295, 1975.

Type species - Corticium gemmiferum Bourdot \& Galzin, Bull. Soc. mycol. Fr. 27(2): 250, 1911.

Basidiome resupinate, effused, adnate, membranaceous to ceraceous, hymenophore smooth, cream to ochraceous. Hyphal system monomitic, hyphae with or without clamps, thin-walled, hyaline. Cystidia (leptocystidia) or cystidiols present, thin-walled, usually apically encrusted with a brown resinous matter. Basidia clavate to suburniform, with 4-sterigmata, and a basal clamp. Basidiospores ellipsoid to navicular, smooth, thin-walled, hyaline, IKI-.

Remarks - Coronicium was originally described to accommodate Corticium gemmiferum, a species with a distinct kind of cystidia apically encrusted with a cap-like of resinous brown substance (Eriksson \& Ryvarden 1975). Jülich (1975) added to deviating species with no encrusted cystidia, Coronicium thymicola and Coronicium alboglaucum, the latter also differing in the simple-septate hyphae. Coronicium belongs to the Pterulaceae in the Agaricales. It is related to Aphanobasidium, Merulicium and Radulomyces. All of them, except Radulomyces, share with Pterula more or less similar ellipsoid to navicular basidiospores (Larsson 2007b).

Corticirama Pilát, Beih. Sydowia 1: 128, 1957.

Type species - Corticirama petrakii Pilát, Beih. Sydowia 1: 128, 1957.

Remarks - A clavarioid genus not included in this manual.

Corticium Pers., Neues Mag. Bot. 1: 110, 1794.

Synonym - Laeticorticium Donk.

Type species - Corticium roseum Pers., Neues Mag. Bot. 1: 111, 1794.

Basidiome resupinate, orbicular or confluent, hymenial surface brightly coloured, smooth. Hyphal system monomitic, generative hyphae with clamps, thin- to thick-walled. Cystidia absent. Dendrohyphidia numerous forming a catahymenium. Basidia large, clavate, cylindrical or tubular, sinuous, thick-walled at the basal part, originating in the subiculum as thick-walled probasidia elongating through a previously developed layer of dendrohyphidia. Basidiospores cylindrical, ovoid to ellipsoid, smooth, thin-walled, IKI-. Spore print pink.

Remarks - Corticium is a well delimited genus after the generic emendation by Boidin \& Lanquetin (1983). It is characterized, among the corticioids with hyaline dendrohyphae, by its not decorticate basidiome growing on dead wood, pink spore-print, and by the absence of cystidial elements. Corticium differs from Dendrocorticium in the thick-walled probasidia, basidiospores usually longer than $12 \mu \mathrm{m}$, and pink spore-print. Laeticorticium is considered a superfluous synonym of Corticium (Boidin \& Lanquetin 1983).

Cotylidia P. Karst., Revue mycol., Toulouse 3(9): 22, 1881.

Type species - Thelephora undulata Fr., Elench. fung. (Greifswald) 1: 164, 1828.

Basidiome pileate, infundibuliform, with a central or lateral stipe, hymenophore smooth to rugose. Hyphal system monomitic, hyphae with simple-septa, thin-walled. Cystidia (pseuodocystidia) with a tramal origin, cylindrical with an obtuse apex, thin-walled, not encrusted. Basidia narrowly clavate, with 4-sterigmata, with a simple basal septum. Basidiospores ellipsoid, smooth, thin-walled, IKI-, CB-.

Remarks - Cotylidia is characterized mainly by the pileate and stipitate stereoid basidiome, the clampless hyphae and the presence of cystidia with tramal origin. Cotylidia is included by molecular data in the Rickenella family under the Hymenochaetales (Larsson 2007b).

Crassisporus B.K. Cui \& Xing Ji, in Ji, Wu, Liu, Si \& Cui, MycoKeys 57: 69, 2019.

Type species - Crassisporus macroporus B.K. Cui \& Xing Ji, MycoKeys 57: 73, 2019.

Basidiome annual, effused-reflexed to pileate, pileal surface pale yellowish brown, yellowish brown to umber-brown when dry, concentrically zonate or sulcate, velutinate, pore surface usually white, cream buff to cinnamon-buff when fresh, buff, pale yellowish brown to yellowish brown 
when dry. Context pale yellowish brown to yellowish brown, leathery to corky when dry. Tubes concolorous with the context, corky when dry. Hyphal system trimitic with clamped generative hyphae, skeletal hyphae hyaline to pale yellowish brown, binding hyphae hyaline to pale yellowish brown, negative in Melzer's reagent, tissues turning to black in KOH. Cystidia absent, thin-walled cystidioles usually present. Basidiospores oblong to broadly ellipsoid, hyaline, smooth, slightly thick-walled, IKI-, CB-. Causing a white rot.

Remarks - Crassisporus is characterized by an annual growth habit, effused-reflexed to pileate basidiocarps with pale yellowish-brown to yellowish brown, concentrically zonate or sulcate, and velutinate pileal surface, a trimitic hyphal system with clamped generative hyphae, tissues turning to dark in $\mathrm{KOH}$, oblong to broadly ellipsoid, hyaline, smooth, and slightly thickwalled basidiospores. Phylogenetic analysis indicates that Crassisporus belongs to the core polyporoid clade grouped with Haploporus forming a monophyletic lineage and related to Perenniporia s.l. (Ji et al. 2019).

Craterella Pers., Neues Mag. Bot. 1: 112, 1794.

Type species - Craterella pallida Pers., Neues Mag. Bot. 1: 112, 1794.

Remarks - A synonym of Cotylidia P. Karst.

Creolophus P. Karst., Meddn Soc. Fauna Flora fenn. 5: 41, 1879.

Type species - Hydnum corrugatum Fr., Observ. mycol. (Havniae) 2: 269, 1818.

Remarks - A synonym of Hericium Pers.

Crepatura C.L. Zhao, in Ma \& Zhao, Mycol. Progr. 18(6): 790, 2019.

Type species - Crepatura ellipsospora C.L. Zhao, in Ma \& Zhao, Mycol. Progr. 18(6): 791, 2019.

Basidiome annual, resupinate. Hymenial surface smooth to irregularly tuberculate, concentrically, and radially cracking. Hyphal system monomitic; generative hyphae thick-walled bearing both clamp connections and simple septa, IKI-, CB-; tissues unchanged in $\mathrm{KOH}$. Cystidia and cystidioles absent; numerous crystals present among the hyphae, basidia clavate, with two sterigmata. Basidiospores ellipsoid, hyaline, thick-walled, smooth, IKI-, CB-.

Remarks - Phylogenetic analyses show that Crepatura belong to the Phanerochaetaceae family and nested into the Donkia clade. Crepatura formed a monophyletic lineage with strong support and phylogenetically was closely related to Pirex concentricus (Ma \& Zhao 2019).

Cristelloporia I. Johans. \& Ryvarden, Trans. Br. mycol. Soc. 72(2): 189, 1979.

Type species - Cristelloporia dimitica I. Johans. \& Ryvarden, Trans. Br. mycol. Soc. 72(2): 189, 1979.

Remarks - A synonym of Trechispora P. Karst.

Cristinia Parmasto, Consp. System. Corticiac. (Tartu): 47, 1968.

Type species - Hydnum helveticum Pers., Mycol. eur. (Erlanga) 2: 184, 1825.

Basidiome resupinate, loosely adnate, hymenophore granular to irregularly raduloid, rhizomorphs present in the subiculum and at the margins. Hyphal system monomitic, hyphae with clamps or simple septa, thin-walled, shortly celled, hyphal strands sometimes present. Cystidia present or absent. Basidia subclavate or cylindrical, with 4-sterigmata, with cyanophilous granulation, with a basal clamp or a simple septum. Basidiospores subglobose to somewhat angular, smooth, thick-walled, IKI-, cyanophilous.

Remarks - Cristinia is characterized above all microscopically, by the basidia with cyanophilous granulation and by the smooth and thick-walled basidiospores. Eriksson \& Ryvarden (1975) suggested some relationships of Cristinia with Hypochnicium due to the similarities of the basidiospores but with differently shaped basidia; with Trechispora by the shape of the basidia while differing in the basidiospores; and with Lindtneria by the cyanophilous granulation of the 
basidia. Molecular data by Binder et al. (2005) suggested the possible relationships with Lindtneria trachyspora and Stephanospora caroticolor, both genera having cyanophilous granulation in the basidia and cyanophilous spore-walls.

Crustoderma Parmasto, Consp. System. Corticiac. (Tartu): 87, 1968.

Type species - Corticium dryinum Berk. \& M.A. Curtis, Grevillea 1(12): 179, 1873.

Basidiome resupinate, effused, ceraceous to crustaceous, closely adnate, hymenophore smooth, yellowish to ochraceous or brown, margin abrupt. Hyphal system monomitic, hyphae with clamps, slightly thick-walled, yellowish. Cystidia cylindrical, thick-walled, smooth or somewhat encrusted. Basidia cylindrical to clavate, hyaline to yellowish, with 4-sterigmata, and a basal clamp. Basidiospores cylindrical to ellipsoid, smooth, thin- to slightly thick-walled, hyaline to yellowish, IKI-, usually stained in CB.

Remarks - Crustoderma is characterized above all by the ceraceous to crustaceous basidiome with yellowish to brownish colours, and microscopically by the large thick-walled cystidia (Nakasone 1984, Parmasto 1968). Crustoderma is classified in the Sparassidaceae, Polyporales (Justo et al. 2017).

Crustodontia Hjortstam \& Ryvarden, Syn. Fung. (Oslo) 20: 36, 2005.

Type species - Corticium chrysocreas Berk. \& M.A. Curtis, Grevillea 1(12): 178, 1873.

Basidiome resupinate, widely effused, closely adnate, hard to brittle, hymenophore smooth, tuberculate to more distinctly odontioid, usually golden to apricot yellow, margin thinning out, usually yellowish, all part of the basidiome turning immediately reddish in $\mathrm{KOH}$. Hyphal system monomitic, hyphae with clamps, thin-walled to moderately thick-walled, hyaline, microbinding hyphae often difficult to detect, but mainly observed in the lower part of the subiculum. Cystidia often numerous, at first sphaeropedunculate then mainly ventricose to ventricose-rostrate, smooth or encrusted beneath the apex. Basidia shortly clavate, hyaline, with 4-sterigmata and with a basal clamp. Basidiospores narrowly ellipsoid to cylindrical, smooth, thin-walled, IKI-, CB-.

Remarks - Crustodontia is closely related to Phlebia but differing in the granular basidiome that becomes reddish in $\mathrm{KOH}$, small ventricose cystidia and small basidiospores (Hjortstam \& Ryvarden 2005). In addition, microbinding skeletoid (fibre-like) hyphae are present in the lower part of the subiculum (this character is also present in the genera Amethicium Hjortstam and Cericium Hjortstam). Molecular data also place Crustodontia in the Meruliaceae close to other phlebioid corticioids (Larsson 2007b).

Crustomyces Jülich, Persoonia 10: 140, 1978.

Type species - Odontia subabrupta Bourdot \& Galzin, Hyménomyc. de France (Sceaux): 430; 1928.

Basidiome annual, resupinate, effused, crustaceous, adnate, hymenophore smooth, odontioid or hydnoid, pale coloured, rhizomorphs or hyphal strands lacking. Hyphal system (mono-) dimitic, generative hyphae hyaline, cylindrical or torulose, with clamps., skeletal hyphae hyaline, about 3 $\mu \mathrm{m}$ in diameter. Cystidia (gloeocystidia) and/or dendrohyphidia present. Basidia clavate, with four sterigmata and a basal clamp. Basidiospores ellipsoid, thin-walled, smooth, hyaline, not amyloid.

Remarks - Crustomyces is morphologically very closely related to Cystostereum sharing the dimitic hyphal system and the presence of gloeocystidia, differing the latter in the absence of dendrophysis (Jülich 1978b). Crustomyces may belong to the Cystosteraceae, where a common family character is the presence of numerous bladder-like gloeocystidia with yellowish contents (Larsson 2007b).

Cryptochaete P. Karst., Bidr. Känn. Finl. Nat. Folk 48: 407, 1889.

Type species - Corticium polygonium Pers., Neues Mag. Bot. 1: 110, 1794.

Remarks - A synonym of Peniophora Cooke. 
Crystallocystidium (Rick) Rick, Brotéria, N.S. 9: 139, 1940.

Basionym - Stereum subgen. Crystallocystidium Rick, Brotéria, N.S. 9: 43, 1940.

Type species - Crystallocystidium vorticosum Rick, Brotéria, N.S. 9: 13, 1940.

Remarks - A name of uncertain application.

Cunninghammyces Stalpers, N.Z. J. Bot. 23(2): 309, 1985.

Type species - Corticium umbonatum G. Cunn., Trans. Roy. Soc. New Zealand 82: 298, 1954.

Basidiome resupinate, effused, pellicular to membranaceous, hymenophore easily separable from subiculum and substrate, smooth. Hyphal system monomitic, hyphae with clamps, hyaline, thin- to somewhat thick-walled. Cystidia absent. Basidia clavate to urniform, thin-walled, hyaline, terminal or usually lateral, with 4-sterigmata, and a basal clamp. Basidiospores globose, echinulate, thin- to slightly thick-walled, often with large globule, non-amyloid.

Remarks - Corticium umbonatum is characterized by the pleurobasidia and thick-walled, globose, echinulate, and inamyloid basidiospores (Stalpers 1985). Hjortstam (1995) synonymized Cunninghammyces to Xenasma although the latter is characterized by the presence of cystidia. Boidin et al. (1986) described Hypochnicium pleurobasidiatum Boidin, Candoussau \& Gilles, refusing to include it in the Xenasmataceae. $H$. pleurobasidiatum was posteriorly synonymized to C. umbonatum by Hjortstam (1995). The phylogenetic relationships of Cunninghammyces are unclear but it is placed in the Cyphellaceae, distantly from other members of Phlebiella (Larsson 2007b). However, it can morphologically be related to Phlebiella, but differing in larger basidia (the presence of pleurobasidia can be only a convergence) and thick-walled basidiospores.

Cyanobasidium Jülich, Persoonia 10(3): 327, 1979.

Type species - Pellicularia chordulata D.P. Rogers, Farlowia 1: 98, 1943.

Remarks - A synonym of Lindtneria Pilát.

Cyanodontia Hjortstam, Mycotaxon 28(1): 23, 1987.

Type species - Cyanodontia spathulata Hjortstam, Mycotaxon 28(1): 23, 1987.

Basidiome resupinate, soft, odontioid to hydnoid, with dense aculei conical or more commonly flattened, or sometimes subporoid especially near the margin. Hyphal system monomitic, subiculum composed by thick-walled hyphae with strongly cyanophilous walls, subhymenial hyphae thin-walled and acyanophilous. Cystidia absent. Basidia short clavate, with 4sterigmate, and a basal clamp. Basidiospores subglobose to ellipsoid, slightly to distinctly thickwalled, with a light cyanophilous reaction, inamyloid, indextrinoid.

Remarks - Cyanodontia reminds Hypochnicium and Nodotia by the thick-walled basidiospores but differing in the light cyanophilous reaction in the basidiospores and distinctly cyanophilous hyphae (Hjortstam 1987b).

Cyanohypha Jülich, Persoonia 11(4): 421, 1982.

Type species - Pellicularia asperula D.P. Rogers, Farlowia 1: 100, 1943.

Remarks - A synonym of Botryobasidium Donk.

Cyanotrama Ghobad-Nejhad \& Y.C. Dai, Mycologia 102(6): 1511, 2010.

Type species - Poria rimosa Murrill, Mycologia 12 (2): 91, 1920.

Basidiome perennial, effused, closely adnate, ceraceous, cream to deep straw. Pores angular, dissepiments finely fluffy under lens, pore surface darkening with $\mathrm{KOH}$, rimose in old specimens. Hyphal system dimitic, generative hyphae with clamps, skeletal hyphae narrow, strongly $\mathrm{CB}+$, IKI-; context pseudodimitic, very thin. Cystidia absent, cystidioles fusoid. Hyphal pegs variably present. Basidia barrel-shaped to short clavate, with four sterigmata and a basal clamp. Basidiospores allantoid, thin-walled, smooth, CB-, IKI-. Causing white rot in conifers especially Juniperus. 
Remarks - Cyanotrama superficially resembles species of Schizopora but it is separated by the allantoid basidiospores, and cyanophilous tramal hyphae. For further comments see GhobadNejhad \& Dai (2010).

Cylindrobasidium Jülich, Persoonia 8: 72, 1974.

Type species - Thelephora evolvens Fr., Observ. mycol. (Havniae) 1: 154, 1815 (=Corticium laeve Pers., Neues Mag. Bot. 1: 110, 1794).

Basidiome resupinate, effuse-reflexed or discoid, hymenophore smooth to tuberculate. Hyphal system monomitic, hyphae with clamps, smooth, thin- to thick-walled, usually with many oil guttules. Cystidia (leptocystidia) fusiform, with a basal clamp. Basidia narrowly clavate, with 4sterigmata and a basal clamp. Basidiospores smooth, thin-walled, non-amyloid.

Remarks - Cylindrobasidium is characterized by the fusiform leptocystidia, narrowly clavate basidia and smooth inamyloid basidiospores (Jülich 1974a). According to molecular data Cylindrobasidium is a member of the Agaricales (Hibbett \& Binder 2002, Larsson et al. 2004, Larsson 2007b).

Cymatoderma Jungh., Tijdschr. Nat. Gesch. Physiol. 7: 290, 1840.

Type species - Cymatoderma elegans Jungh., Tijdschr. Nat. Gesch. Physiol. 7: 290. 1840.

Basidiome coriaceous, dimidiate, flabellate and pseudoinfundibuliform, frequently becoming confluent, with short lateral or long pseudocentral stipe. Pileus often completely covered by a thick felt-like tomentum. Hymenial surface varying from beige, ochraceous-beige or pinkish-brown to ochraceous-brown (rarely dark purplish-brown in very old material), and ornamented with rather prominent, branched, radiating folds which bear scanty or densely crowded warts or aculei. Hyphal system dimitic, generative hyphae thin- to thick-walled, clamped, skeletal hyphae, often with thinwalled apices, hyaline to brownish. Cystidia present as apically encrusted metuloids and gloeocystidia often abundant. Basidia clavate, usually 4-spored but sometimes 2-spored. Basidiospores hyaline, broadly elliptical to subglobose, thin-walled, inamyloid.

Remarks - Cymatoderma is a stipitate genus with pantropical distribution (Junghuhn 1840).

Cyphellostereum D.A. Reid, Nova Hedwigia, Beih. 18: 336, 1965.

Type species - Stereum pusiolum Berk. \& M.A. Curtis, J. Linn. Soc., Bot. 10(46): 330, 1868.

Basidiome pileate, dimidiate to flabelliform, with a rudimentary stipe but often inconspicuous, hymenophore smooth. Hyphal system monomitic, hyphae without clamps, thinwalled, and with a lichenized structure. Cystidia absent. Basidia clavate, with 4-sterigmata and a simple basal septum. Basidiospores ellipsoid to subglobose, smooth, thin-walled, IKI-.

Remarks - Cyphellostereum is characterized by the flabelliform basidiome with a liquenized structure and lack of cystidial elements. It belongs to the Agaricales (Lawrey et al. 2009). Cantharellus laevis was previously placed in Cyphellostereum, but it differs phylogenetically, and morphologically, in the presence of cystidia and presenting a non-liquenized structure. Cantharellus laevis is now placed in the genus Muscinupta.

Cystidiodendron Rick, Lilloa 9: 218, 1943.

Type species - Cystidiodendron fimbriatum Rick, Lilloa 9: 21, 1943 (=Fibrodontia gossypina Parmasto, Conspec. System. Corticiacearum: 207, 1968).

Remarks - According to Baltazar et al. (2016), Cystidiodendron fimbriatum Rick is conspecific and a previous synonym of Fibrodontia gossypina Parmasto. Thus, Fibrodontia Parmasto (1968) should be considered a latter synonym of Cystidiodendron Rick (1943), sharing both genera the same generic type (Rick 1943). Baltazar et al. (2016) combined Cystidiodendron fimbriatum Rick in Fibriporia as Fibrodontia fimbriata (Rick) Baltazar \& Rajchenb.

Cystidiodontia Hjortstam, Mycotaxon 17: 571, 1983. emend. Hjortstam \& Ryvarden, Mycotaxon 25(2): 546, 1986. 
Type species - Hydnum artocreas Berk. \& M.A. Curtis, Grevillea 20(no. 93): 1, 1891 (=Hydnum laminiferum Berk. \& M.A. Curtis, J. Linn. Soc., Bot. 10(no. 46): 325, 1868).

Basidiome resupinate, closely adnate, crustaceous, hymenophore grandinioid, odontioid or hydnoid. Hyphal system dimitic, skeletal hyphae indextrinoid or strongly dextrinoid, without clamps, subhymenial hyphae thin-walled or with slight wall thickening, with clamps. Gloeocystidia as a rule numerous, with yellowish contents, SA-. Dendrohyphidia in the hymenial layer lacking or present. Basidia subclavate, small to medium-sized, more or less constricted, with 4 sterigmata, and a basal clamp. Basidiospores moderately thick-walled, subglobose, fairly small, indextrinoid, inamyloid but with a slight cyanophilous reaction.

Remarks - Cystidiodontia seems very closely related to Crustomyces, sharing the dimitic hyphal system and the presence of dendrohyphidia and gloeocystidia, but basidiospores are thickwalled and skeletal hyphae stain reddish-brown in Melzer's reagent in some species of Cystidiodontia. The genus was described with Hydnum artocreas Berk. \& M.A. Curtis as the generic type species, but the description was based on East African specimens which later on appeared to be distinctly separated from the type of $\mathrm{H}$. artocreas belonging to Kneiffia isabellina Berk. \& Br., which primarily differs from its dextrinoid skeletal hyphae (Hjortstam \& Ryvarden 1986).

Cystostereum Pouzar, Česká Mykol. 13: 18, 1959.

Type species - Thelephora murrayi Berk. \& M.A. Curtis [as 'murraii'], J. Linn. Soc., Bot. 10(46): 329, 1868.

Basidiome perennial, resupinate, effused, crustaceous, adnate, hymenophore tuberculate to odontioid, whitish, rhizomorphs or hyphal strands lacking. Hyphal system dimitic, generative hyphae with clamps, hyaline, richly branched, and thick-walled fibre-like skeletal hyphae. Gloeocystidia or gloeocystidioid vesicles numerous. Dendrohyphidia absent. Basidia clavate, with 4-sterigmata and a basal clamp. Basidiospores ellipsoid, thin-walled, smooth, hyaline, not amyloid.

Remarks - Cystosterum is characterized by the dimitic hyphal system and by the numerous vesicular gloeocystidia. It differs from Crustomyces mainly in the absence of dendrophyses. Both are molecularly classified in the Cystosteraceae (Larsson 2007b).

Cytidia Quél., Fl. mycol. France (Paris): 25, 1888.

Type species - Thelephora salicina Fr., Syst. mycol. (Lundae) 1: 442, 1821.

Basidiome resupinate at first to typically cupuliform, subgelatinosus when fresh and coriaceous when dry, hymenophore smooth, reddish to violaceous. Hyphal system monomitic, hyphae with clamps at all septa, thin to thick-walled, bluish in sulfovainilline. Cystidia absent. Dendrohyphidia present, light brown. Basidia large, claviform, with 4-sterigmata and a basal clamp. Basidiospores allantoid, smooth, IKI-, CB-.

Remarks - Cytidia seems to be related with other genera in the Corticiaceae s.str., such as Corticium, Dendrocorticium and Vuilleminia (Hallenberg \& Parmasto 1998, Larsson 2007b). All of them, growing on branches, share the particular habitat, the presence of dendrohyphidia, and the large basidiospores. Cystidia is mainly characterized by the reddish subgelatinous cupuliform basidiome, brown dendrohyphidia, and the absence of any cystidial element.

Cytidiella Pouzar, Česká Mykol. 8: 128, 1954.

Type species - Cytidiella melzeri Pouzar, Česká Mykol. 8: 129, 1954. (=Cytidia albomellea Bondartsev, Morbi plant Leningrad 16(1): 96, 1927).

Remarks - A synonym of Auriculariopsis Maire (Kotlába 1988).

Dacrina Fr., Syst. orb. veg. (Lundae) 1: 172, 1825.

Type species - Hydnum sudans Alb. \& Schwein., Consp. fung. (Leipzig): 272. 1805.

Remarks - A synonym of Dacryobolus Fr. 
Dacryobasidium Jülich, Biblthca Mycol. 85: 396, 1982.

Type species - Corticium coprophilum Wakef., Trans. Br. mycol. Soc. 5(3): 480, 1916.

Remarks - A synonym of Cristinia Parmasto.

Dacryobolus Fr., Summa veg. Scand. (Stockholm) 2: 404, 1849.

Type species - Hydnum sudans Alb. \& Schwein., Consp. fung. (Leipzig): 272, 1805.

Basidiome resupinate, adnate, effused, membranaceous to coriaceous, hymenophore smooth, odontioid or tuberculate, margin undifferentiated or finely fibrillose. Hyphal system monomitic or dimitic, hyphae hyaline, thin-walled or slightly thick-walled, generative hyphae clamped. Cystidia present as pseudocystidia and hymenial cystidia. Basidia long, narrow, cylindrical to subclavate, with the apical part constricted close to the sterigmata, thin-walled, with 4-sterigmata and a basal clamp. Basidiospores allantoid, smooth, IKI-.

Remarks - Dacryobolus is characterized by the long and narrow basidia, the allantoid basidiospores and the cystidia with tramal origin that project conspicuously above the basidial layer. Parmasto (1968) and Eriksson \& Ryvarden (1975) were in agreement in the relationship of Dacryobolus with the phlebioid corticioids. Molecular analysis by Binder et al. (2005) placed Dacryobolus in the Antrodia clade, close to other polyporoid genera as Antrodia, Auriporia, Amylocystis and Oligoporus.

Datronia Donk, Persoonia 4(3): 337, 1966.

Type species - Daedalea mollis Sommerf., Suppl. Fl. lapp. (Oslo): 271, 1826.

Basidiome annual, wide effused-reflexed (with the effused part strongly adnate) or sessile, to pileate (in Podofomes trogii), frequently confluent, cover of pileus velutinous-tomentosus, zonate, usually brown to black, hymenophore tubular, in a solitary layer, small or medium-sized, later often with irregular pores, trama thin, pale-brown, somewhat coriaceous, separated from the tomentosus cover by a thin, black layer. Hyphal system dimitic, generative hyphae thin-walled, hyaline, nodose-septate, skeletal hyphae thick-walled, non-septate, with pale walls, some of them thinner, occasionally dendritically branched hyphae of tomentosus cover with thick, intensively colored walls. Cystidia absent, cystidioles may be present. Basidia clavate, tetrasterigmate, with a basal clamp. Basidiospores cylindrical, medium-sized (8-11 $\mu \mathrm{m}$ long), with thin, hyaline, smooth, nonamyloid walls. Causing a white rot.

Remarks - Datronia is characterized by the brown to the black cuticle and the dendroid hyphae in the dissepiments. Datronia is phylogenetically classified in the polyporoid clade, with some species of Polyporus in the 'Datronia clade' (Justo \& Hibbett 2011). Daedalea mollis Sommerf., the generic type of Datronia is nested with Boletus squamosus Huds., and some other species in Polyporus in the so-called 'Datronia clade' by Justo \& Hibbett (2011). So, Datronia is considered a synonym of Cerioporus Quél. according to Zmitrovich \& Kovalenko (2016). However, the phylogeny of Polyporus and allied genera is not fully resolved, and we prefer to keep apart Datronia s.str., Datroniella and Neodatronia in a more restrictive consideration of the group according to Li et al. (2014). Podofomes trogii (Fr.) Pouzar, a stipitate polypore, also belongs to the Datronia s.str. clade (Gorjón, unpublished).

Datroniella B.K. Cui, Hai J. Li \& Y.C. Dai, in Li, Cui \& Dai, Persoonia 32: 172, 2014.

Type species - Polyporus scutellatus Schwein., Trans. Am. phil. Soc., New Series 4(2): 157, 1832.

Basidiome annual, pileate, effused-reflexed or rarely resupinate, when pileate, pileus usually projecting less than $3 \mathrm{~cm}$, pileal surface brown to black, glabrous, pore surface white, cream to pale brown, pores large to small, round to angular. Context pale brown to brown, corky. Hyphal system dimitic, generative hyphae with clamp connections, skeletal hyphae usually dominating, pale brown to brown, moderately to frequently branched in context and frequently branched in trama, IKI-, $\mathrm{CB}+$, tissues darkening in $\mathrm{KOH}$. Dendrohyphidia and cystidia absent in the hymenium, thin-walled 
cystidioles usually present. Basidiospores cylindrical, hyaline, thin-walled, smooth, IKI-, CB-. Usually growth on angiosperm wood and causing a white rot.

Remarks - Datroniella is phylogenetically, closely related to Datronia, and morphologically they differ by the moderately to frequently branched skeletal hyphae in context and absence of dendrohyphidia in the dissepiments in the former ( $\mathrm{Li}$ et al. 2014).

Dendrocorticium M.J. Larsen \& Gilb., Norw. Jl Bot. 21: 225, 1974.

Type species - Corticium polygonioides P. Karst., Meddn Soc. Fauna Flora fenn. 6: 12, 1881.

Basidiome annual, resupinate or effuse-reflexed, hymenial surface smooth, margin thinning out. Hyphal system monomitic, hyphae with clamps, thin- to thick-walled. Dendrohyphidia present, with or without a distinct and well-defined catahymenium. Cystidia absent. Basidia developed from thin-walled probasidia originating in the subhymenium, mature basidia formed by intrusive metabasidial growth, with 4-sterigmata and a basal clamp. Basidiospores subglobose, ellipsoid or broadly ovoid, smooth, thin-walled, IKI-, spore print white.

Remarks - Dendrocorticium is very close related to Corticium, both share the presence of hyaline dendrophyses, lack of cystidial elements and the smooth inamyloid basidiospores, but differing in the thin-walled probasidia and in the white spore print in the former (Larsen \& Gilbertson 1974). Phylogenetically, both are classified in the Corticiaceae (Larsson 2007b).

Dendrodontia Hjortstam \& Ryvarden, Mycotaxon 10(2): 273, 1980.

Type species - Grandinia bicolor P.H.B. Talbot, Bothalia 4(4): 947. 1948.

Basidiome resupinate, effuse, loosely adnate, hymenial surface tuberculate or odontioid, yellowish to greyish, subiculum well differentiated, brownish. Hyphal system dimitic (or pseudodimitic), generative hyphae with clamps, thin- to thick-walled, skeletal or pseudoskeletal hyphae thick-walled, yellowish to brown, slightly ramified, with sparse clamps. Dendrohyphidia hyaline. Cystidia absent. Basidia clavate, terminal, with 4-sterigmata and a basal clamp. Basidiospores ellipsoid to suballantoid, smooth, thin-walled, IKI-, CB-.

Remarks - Dendrodontia is characterized by the short basidia (without a probasidial stage), dendrohyphae, presence of brown skeletal hyphae in the subiculum (can be considered pseudoskeletal hyphae because they have sparse clamps), and small basidiospores (Hjortstam \& Ryvarden 1980a). It seems morphologically related to Dentocorticium, but it differs above all in the presence of skeletoid hyphae. Binder et al. (2005) pointed a phylogenetic relationship of a Dendrodontia sp. with Dentocorticium sulphurellum (Peck) Larsen \& Gilb., also with skeletal hyphae in the context, a species that Boidin \& Gilles (1998) have already been transferred to Dendrodontia based on morphological characters. However, the type species of Dentocorticum, Dentocorticium ussuricum (Parmasto) Larsen \& Gilb., with a monomitic hyphal system, is not still sequenced, and phylogenetic relationships between Dendrodontia and Dentocorticium are still unclear. Brunneocorticium is somewhat similar differing in the smooth hymenial surface, presence of leptocystidia with some secondary simple septa (even they are not conspicuous and are difficult to detect), and bisterigmate basidia. Also, the type species of Brunneocorticium, cluster in the Agaricales clade and a Dendrodontia sp. in the polyporoid clade.

Dendrominia Ghobad-Nejhad \& Duhem, Mycological Progress 13: 7, 2014.

Type species - Aleurocorticium maculatum H.S. Jacks. \& P.A. Lemke in Lemke, Can. J. Bot. 42: 742, 1964.

Basidiome resupinate, smooth, decorticating or not, closely adnate, white to whitish cream, ceraceous to crustaceous, surface finely farinose, margin distinct. Hyphal system monomitic, hyphae with or without clamps, narrow, thin-walled, encrusted with fine amorphous crystals. Subiculum lacking. Basidia large, long cylindrical clavate, flexuous, arising from inflated basidioles (probasidia), with four stout sterigmata, with or without a basal clamp, contents granular. Cystidia lacking. Dendrohyphidia present. Basidiospores lunate to allantoid, contents granular, smooth, thin-walled, CB-, IKI-. 
Remarks - Dendrominia was created to accommodate some species previously placed in Dendrothele or Vuilleminia, with intermediate features but phylogenetically well separated. Dendrothele species grow on the bark, have cystidia and cyanophilous, thick-walled basidiospores, while Dendrominia species may grow on bark or not, lack cystidia, and have thin-walled, CBbasidiospores. Dendrominia differs from Vuilleminia by its basidiome texture (ceraceous to crustaceous in the former, mostly gelatinous in the latter) and lack of clamp in some of its species (Ghobad-Nejhad \& Duhem 2014).

Dendrophlebia Dhingra \& Priyanka, Mycotaxon 116: 157, 2011.

Type species - Dendrophlebia crassispora Dhingra \& Priyanka, Mycotaxon 116: 159, 2011.

Basidiome resupinate, closely adnate, effused, ceraceous, hymenial surface smooth, continuous, yellowish to brownish, turning dark ruby on $3 \% \mathrm{KOH}$ solution, margin not differentiated. Hyphal system monomitic, generative hyphae branched, clamped, hyphae often agglutinated, penetrating deep into the substratum, covered by some yellowish-browncrystalline matter. Dendrohyphidia present. Cystidia thin-walled, hyphoid. Basidia clavate to subclavate, 4sterigmate, with a basal clamp. Basidiospores ellipsoid, smooth, with thickened walls, inamyloid, CB-.

Remarks - Dendrophlebia resembles Phlebia in having a compact agglutinated texture, clavate basidia, clamped generative hyphae, and 4-sterigmate basidia (Dhingra \& Priyanka 2011a). The presence of dendrohyphidia and basidiospores with distinctly thickened walls distinguish Dendrophlebia from Phlebia. The reaction with $\mathrm{KOH}$, changing to red the hymenial surface, is also present in the phlebioid genus Crustodontia.

Dendrophora (Parmasto) Chamuris, Mycotaxon 28: 543, 1987.

Basionym - Peniophora subgen. Dendrophora Parmasto, Consp. System. Corticiac. (Tartu): 131, 1968.

Type species - Stereum versiforme Berk. \& M.A. Curtis, Grevillea 1(11): 164, 1873.

Basidiome resupinate, effuse-reflexed or rarely sessile. Hymenophore smooth, finely pruinose or velutinous, grey to brown, abhymenial surface brown to black, tomentose to felty, zonate or not. Hyphal system monomitic (or pseudodimitic), hyphae with clamp, subhyaline to brown, thin- to thick-walled. Cystidia (lamprocystidia) present, heavily encrusted. Dendrohyphidia present, subhyaline to brown. Basidia narrowly clavate, with 4-sterigmata, and with a basal clamp. Basidiospores cylindrical to allantoid, smooth, thin-walled, non-amyloid.

Remarks - Dendrophora species are quite similar to species of Peniophora and traditionally they have been included in the latter. Dendrophora mainly differs in the brown dendrohyphidia. It is phylogenetically classified in the Peniophoraceae (Larsson 2007b).

Dendrophysellum Parmasto, Consp. System. Corticiac. (Tartu): 146, 1968.

Type species - Dendrophysellum amurense Parmasto, Consp. System. Corticiac. (Tartu): 206, 1968.

Remarks - A synonym of Vararia P. Karst. See Nakasone (2015) for further comments.

Dendrothele Höhn. \& Litsch., Sber. Akad. Wiss. Wien, Math.-naturw. Kl., Abt. 1 116: 819, 1907.

Type species - Dendrothele papillosa Höhn. \& Litsch., Sber. Akad. Wiss. Wien, Math.naturw. Kl., Abt. 1 116: 820, 1907 (= Corticium griseocanum Bres., Fung. trident. 2(11-13): 58, 1898).

Basidiome usually orbicular, discoid to corticioid, whitish to pale ochraceous, hymenophore smooth to tuberculate or papillose, margin usually determinate and abrupt. Hyphal system monomitic, hyphae with or without clamps, very difficult to discern due to the crystalline matter. Cystidia present or absent. Dendrohyphidia numerous, encrusted. Basidia large, broadly cylindrical to clavate, with (2-3)4-sterigmata. Basidiospores thin- or slightly thick-walled, usually smooth or warted, non or slightly amyloid, usually with cyanophilous walls. 
Remarks - Dendrothele appears to be a heterogeneous genus and several molecular analyses showed it is highly polyphyletic (Goranova et al. 2003, Bodensteiner et al. 2004, Binder et al. 2005, Larsson 2007b). It is characterized by growing on the bark of living trees (only there are few records of Dendrothele species on decayed wood), the usually orbicular basidiome and microscopically by the presence of numerous dendrophyses, crystals in the context, and by the large basidiospores with distinct and usually cyanophilous walls (Höhnel \& Litschauer 1907, Gorjón \& Bernicchia 2010). It is included in the Lachnellaceae Boud., in an isolated position among the corticioid fungi, close to other species within the Agaricales (Larsson 2007b).

Dentipellicula Y.C. Dai \& L.W. Zhou, Mycologia 105(3): 639, 2013.

Type species - Dentipellicula taiwaniana (Sheng H. Wu) Y.C. Dai \& L.W. Zhou, Mycologia 105(3): 641, 2013.

Basidiome annual, effused-reflexed to pileate, hymenophore hydnoid, aculei soft corky, cream to ivory yellow when fresh, leathery, buff to cinnamon buff when dry. Margin cottony, cream, narrow. Subiculum soft corky, white to pallid. When pileate, pileal surface cinnamon-buff, buff to cream toward margin, indistinctly concentrically zonate, glabrous; margin sharp, incurved when dry. Hyphal system monomitic; generative hyphae with clamp connections, IKI-, CB-. Gloeoplerous hyphae absent or present. Gloeocystidia present; cystidia absent. Basidiospores slightly thick-walled, hyaline, minutely rough, strongly amyloid, CB-.

Remarks - Dentipellicula is morphologically similar to Dentipellis but differs in the CBgenerative hyphae (Zhou \& Dai 2013).

Dentipellis Donk, Persoonia 2(2): 232, 1962.

Type species - Hydnum fragile Pers., Syn. meth. fung. (Göttingen) 2: 561, 1801.

Basidiome resupinate, hymenophore hydnoid. Hyphal system monomitic, hyphae with clamps, $\mathrm{CB}+$. Gloeocystidia present. Basidia clavate, with 4-sterigmata and a basal clamp. Basidiospores subglobose to globose, finely asperulate, amyloid.

Remarks - Dentipellis is characterized by the hydnoid hymenophore, monomitic hyphal system, presence of gloeocystidia and ornamented amyloid basidiospores. It is related to other genera in the Hericiaceae as Laxitextum and Hericium, sharing the gloeocystidia and the ornamented amyloid basidiospores; molecular data also confirm these relationships (Larsson 2007b).

Dentipellopsis Y.C. Dai \& L.W. Zhou, Mycologia 105(3): 642, 2013.

Type species - Dentipellopsis dacrydicola Y.C. Dai \& L.W. Zhou, Mycologia 105(3): 642, 2013.

Basidiome annual, resupinate, hymenophore hydnoid, aculei soft, cream to flesh-pink when fresh, fragile, cream when dry, clay-buff in bruised part, margin cottony, white, narrow. Subiculum soft corky, white to cream. Hyphal system monomitic; generative hyphae with clamp connections, IKI-, CB-. Gloeoplerous hyphae absent in subiculum, present in hymenophoral trama. Gloeocystidia and cystidia present. Basidiospores distinctly thick-walled, colorless, densely echinulate but smooth in $\mathrm{KOH}$, strongly amyloid, $\mathrm{CB}+$.

Remarks - Dentipellopsis is morphologically similar to Dentipellis and Dentipellicula but differs in the distinctly thick-walled basidiospores. Phylogenetically it also seems quite isolated from previous genera (Zhou \& Dai 2013).

Dentiporus Audet, Mushrooms nomenclatural novelties 3: 1, 2017.

Type species - Antrodia albidoides A. David \& Dequatre, Mycol. helv. 1(6): 361, 1985.

Remarks - According to Audet (2017c) Dentiporus differs from Antrodia s.str. by smaller basidiomes, only resupinate, relatively soft, thicker dissepiments in young basidiomata becoming irpicoid with age, skeletal hyphae with capillary to distinct lumen, uninucleate and cylindrical basidiospores. 
Dentipratulum Domański, Acta Mycologica, Warszawa 1: 6, 1965.

Type species - Dentipratulum bialoviesense Domański, Acta Mycologica, Warszawa 1(6): 7, 1965.

Basidiome as individual gregarious aculei, pointed and unbranched. Hyphal system monomitic, generative hyphae with clamps. Gloeocystidia present. Basidia clavate, with 4sterigmata and a basal clamp. Basidiospores subglobose to globose, smooth to finely asperulate, amyloid.

Remarks - Dentipratulum is characterized macroscopically by the basidiome formed by gregarious aculei. It is morphologically related to Mucronella that differs in the absence of gloeocystidia. Dentipratulum is phylogenetically included among the russuloid lineage, with Gloiodon and Auriscalpium as the closest related genera (Larsson \& Larsson 2003, Larsson 2007b).

Dentocorticium (Parmasto) M.J. Larsen \& Gilb., Norw. J Bot. 21: 225, 1974.

Basionym - Laeticorticium sect. Dentocorticium Parmasto, Consp. System. Corticiac. (Tartu): 151, 1968.

Type species - Laeticorticium ussuricum Parmasto, Eesti NSV Tead. Akad. Toim., Biol. Seer 14(2): 229, 1965.

Basidiome annual, effused, hymenial surface smooth and undulating or odontioid. Hyphal system monomitic or dimitic, subicular hyphae normally clamped and with some wall thickening. Dendrohyphidia present, with or without a distinct and well-defined catahymenium. Probasidia absent. Basidia clavate, 4-sterigmata, 30-50 $\mu \mathrm{m}$ long. Basidiospores rarely wider than $4 \mu \mathrm{m}$, ellipsoid to cylindrical, allantoid, hyaline, IKI-, CB-.

Remarks - Dentocorticium differs from Corticium and Dendrocorticium in the absence of probasidia, smaller basidiospores, and in the hymenophore more or less odontioid (Larsen \& Gilbertson 1974).

Deviodontia (Parmasto) Hjortstam \& Ryvarden, Syn. Fung. (Oslo) 26: 49, 2009.

Basionym - Hyphodontia sect. Deviodontia Parmasto, Consp. System. Corticiac. (Tartu): 125, 1968.

Type species - Odontia pilaecystidiata S. Lundell, Fungi Exsiccati Suecici 43-44: 24, 1953.

Basidiome resupinate, adnate, hymenophore hydnoid to raduloid with conical to cylindrical aculei. Hyphal system monomitic, hyphae with clamps, thin- to slightly thick-walled. Cystidia numerous, distinctly capitate, thin-walled. Basidia clavate, constricted, with 4-sterigmata and a basal clamp. Basidiospores ellipsoid, smooth, thin-walled, IKI-, CB-.

Remarks - Deviodontia is a monotypic genus, segregated from Hyphodontia s.l. on the basis of its characteristic large cylindrical cystidia with capitate apex. According to Yurchenko et al. (2020), Deviodontia is a synonym of Kneiffiella.

Dextrinocystidium Sheng H. Wu, Mycologia 87(6): 888, 1996.

Type species - Peniophora sacrata G. Cunn., Trans. Roy. Soc. N.Z. 83: 274, 1955.

Remarks - A synonym of Gloeopeniophorella according to Hjortstam \& Ryvarden (2007). It differs from Gloeopeniophorella in the dextrinoid lamprocystidia (see Wu 1995).

Dextrinocystis Gilb. \& M. Blackw., Mycotaxon 33: 376, 1988.

Type species - Epithele capitata D.P. Rogers \& Boquiren, Mycologia 63: 942, 1971.

Basidiome resupinate, effused, soft, hymenial surface smooth, tomentose under the lens, pale ochraceous to buff. Hyphal system monomitic, generative hyphae thin-walled, with clamps, some with cystidiate straight branches apically encrusted with an umbrella-like matter. Cystidia abundant, tubular, with a branched base, thick-walled with a narrow lumen, apically with an umbrella-like encrustation, strongly dextrinoid except in the apical part, not dissolving in $\mathrm{KOH}$. Basidia clavate to utriform, with 4-sterigmata, and a basal clamp. Basidiospores cylindrical to slightly curved, smooth, hyaline, inamyloid, indextrinoid. 
Remarks - Dextrinocystis is characterized by the dextrinoid (lyo) cystidia with an umbrellalike encrustation not dissolving in KOH. The only species, Dextrinocystis capitata (D.P. Rogers \& Boquiren) Gilb. \& M. Blackw., is reminiscent of Tubulicrinis hamatus (H.S. Jacks.) Donk, but differs in the dextrinoid reaction of the cystidia (Gilbertson \& Blackwell 1988). Many species of Tubulicrinis have lyocystidia that do not dissolve completely or clearly in $\mathrm{KOH}$, so the concept of the lyocystidium as a cystidium that dissolves in $\mathrm{KOH}$ should be taken with precaution.

Dextrinodontia Hjortstam \& Ryvarden, Mycotaxon 12: 172, 1980. 1980.

Type species - Dextrinodontia molliuscula, Hjortstam \& Ryvarden, Mycotaxon 12: 173,

Basidiome resupinate, effuse, hymenophore distinctly hydnoid, faily soft. Hyphal system dimitic, skeletal hyphae thick-walled, sparsely ramified, clearly dextrinoid and with a strong cyanophilous reaction, without clamps, generative hyphae thin-walled, with clamps at all septa. Cystidia absent. Basidia more or less clavate, with 4-sterigmata, with a basal clamp. Basidiospores subglobose, smooth, thin-walled, inamyloid.

Remarks - Dextrinodontia is characterized by the hydnoid basidiome, dextrinoid and cyanophilous hyphae, and smooth basidiospores (Hjortstam \& Ryvarden 1980b). It was considered a synonym of Trechispora by Larsson (1992).

Dichomitus D.A. Reid, Rev. Biol., Lisb. 5(1-2): 149, 1965.

Type species - Trametes squalens P. Karst., in Rabenhorst \& Winter, Fungi Europ. Extra-Eur. exsicc.: no. 3528, 1886.

Basidiome annual to perennial, resupinate to pileate and broadly sessile; upper surface white to blackish, pore surface white, cream to pale grayish, in some specimens with a darkened margin. Pores small to large, context white to cream. Hyphal system dimitic, generative hyphae with clamps, skeletal hyphae dendritically branched, with tapering ends. Cystidia none. Basidia clavate, 4-sterigmate, with a basal clamp. Basidiospores cylindrical to oblong ellipsoid, smooth, hyaline, thin-walled, negative in Melzer's reagent. On dead wood of gymnosperms and angiosperms, causing a white rot.

Remarks - Dichomitus is in some respects similar to Polyporus, and the two genera are closely related. The main characters separating them are the stipitate to dimidiate (or fan-shaped) basidiomes of Polyporus and the resupinate to broadly sessile ones of Dichomitus. See also Ryvarden (1991), Ryvarden \& Melo (2017) and Bernicchia \& Gorjón (2020).

Dichostereum Pilát, Annls mycol.: 223, 1926.

Type species - Asterostromella dura Bourdot \& Galzin, Bull. Soc. mycol. Fr. 36: 74, 1920.

Basidiome resupinate, effused, membranaceous to crustaceous, hymenophore smooth to grandinioid, usually cream to ochraceous. Hyphal system dimitic with clamped generative hyphae and dichohyphae. Gloeocystidia present, SA+ in most species. Basidia narrowly clavate, with 4sterigmata, and a basal clamp. Basidiospores subglobose to broadly ellipsoid, verrucose, with a strong amyloid reaction.

Remarks - Dichostereum is characterized by the dextrinoid dichohyphae, presence of gloeocystidia, and amyloid basidiospores. It is closely related to Vararia, but mainly separated by the ornamented basidiospores. Phylogenetically both are classified in the Peniophoraceae among the russuloid clade (Larsson \& Larsson 2003, Larsson 2007b).

Dichopleuropus D.A. Reid, Nova Hedwigia, Beih. 18: 329, 1965.

Type species - Dichopleuropus spathulatus D.A. Reid, Nova Hedwigia, Beih. 18: 329, 1965.

Basidiome terrestrial, coriaceous, spathulate to flabelliform, pileal surface glabrous to rugulose, hymenial surface smooth, stipe lateral, widened towards the pileus. Hyphal system dimitic, generative hyphae simple-septate, binding hyphae dichotomously branched, thick-walled, 
yellowish-brown, dextrinoid. Gloeocystidia present. Basidia clavate, with 4-sterigmate, simpleseptate at the base. Basidiospores ellipsoid to subglobose, smooth, thin-walled, hyaline, amyloid.

Remarks - Dichopleuropus is characterized by the pileate and stipitate basidiomes, dextrinoid dichotomously branched binding hyphae, and amyloid globose basidiospores. The genus seems to be related to other genera in the Lachnocladiaceae (Reid 1965).

Diplomitoporus Domański, Acta Soc. Bot. Pol. 39: 191, 1970.

Type species - Trametes flavescens Bres., Annls mycol. 1(1): 81, 1903.

Basidiome annual, resupinate to effused reflexed, white to light-coloured. Pores circular to angular medium to small. Hyphal system dimitic, generative hyphae with clamps, skeletal hyphae hyaline, thick-walled, non-amyloid. Cystidia absent or present. Basidia clavate, 4-sterigmate, with a basal clamp. Basidiospores allantoid to ellipsoid, thin-walled, smooth, negative in Melzer's reagent. On dead wood of conifers and hardwoods. Causing a white rot.

Remarks - Diplomitoporus is in some respects similar to some species of Antrodia, but mainly differing in the type of rot, brown rot in Antrodia and usually with larger basidiospores. It is also similar to Antrodiella, but the latter usually has smaller basidiospores and with a more ellipsoid shape. See also Ryvarden (1991), Ryvarden \& Melo (2017) and Bernicchia \& Gorjón (2020).

Diplonema P. Karst., Bidr. Känn. Finl. Nat. Folk 48: 430, 1889.

Type species - Diplonema sordescens P. Karst., Bidr. Känn. Finl. Nat. Folk 48: 430, 1889.

Remarks - A latter homonym of Diplonema G. Don 1837 (Ebenaceae) and a synonym of Amphinema P. Karst.

Donkia Pilát, Bull. trimest. Soc. mycol. Fr. 52(3): 328, 1937.

Type species - Hydnum pulcherrimum Berk. \& M.A. Curtis, Hooker's J. Bot. Kew Gard. Misc. 1: 235, 1849.

Remarks - Treated as a synonym of Climacodon P. Karst., but Hydnum pulcherrimum, that clusters in the vicinity of other species of Phanaerochaete, probably merits a genus of its own as suggested by Moreno et al. (2007) based on morphology and phylogenetic characters.

Donkioporia Kotlába \& Pouzar, Persoonia 7: 214, 1973.

Type species - Boletus expansus Desm., Catal. des plantes omis.: 19, 1823.

Basidiome resupinate, perennial, woody, dark brown. Pores small, context brown, separated from the substrate by a black line. Hyphal system trimitic, generative hyphae with clamps, skeletal hyphae pale brown, binding hyphae hyaline. Cystidia none. Basidia clavate, with 4-sterigmate and a basal clamp. Basidiospores ellipsoid, smooth, thin-walled, hyaline, negative in Melzer's reagent. On hardwoods, causing a white rot.

Remarks - Superficially, Donkioporia resembles a resupinate Phellinus species but the clamps on the generative hyphae immediately exclude this genus. The brown tissue and clamped generative hyphae are characters also found in Gloeophyllum. However, Gloeophyllum species cause a brown rot, have cylindrical spores and most species also have cystidia (Kotlába \& Pouzar 1973, Bernicchia \& Gorjón 2020).

Dryodon Quél. ex P. Karst., Revue mycol., Toulouse 3(no. 9): 19, 1881.

Type species - Hydnum coralloides Scop., Fl. carniol., Edn 2 (Wien) 2: 472, 1772.

Remarks - A synonym of Hericium Pers.

Duportella Pat., Philipp. J. Sci., C, Bot. 10: 87, 1915.

Type species - Duportella velutina Pat., Philipp. J. Sci., C, Bot. 10(2): 87, 1915.

Basidiome resupinate, effused to effuse-reflexed, adnate, hymenophore smooth, greyish, reddish, violaceous, ochraceous or blackish. Hyphal system monomitic or dimitic, hyphae usually with clamps or some simple-septa. Cystidia of two kinds: 1) pseudocystidia, brown, apically 
encrusted, 2) gloeocystidia, usually SA+. Basidia cylindrical to subclavate, with 4-sterigmata, and with a basal clamp. Basidiospores variable in shape and characteristic for species delimitation, ellipsoid to ovoid or globose, cylindrical to allantoid, smooth, thin-walled, hyaline, IKI-.

Remarks - Duportella is characterized by Peniophora s.l. by the brown encrusted pseudocystidia and by presence of skeletal or skeletoid hyphae (Andreasen \& Hallenberg 2009). Duportella is closely related to Peniophora and Dendrophora and it is classified in the Peniophoraceae (Larsson 2007b).

Earliella Murrill, Bull. Torrey bot. Club 32(9): 478, 1905.

Type species - Earliella cubensis Murrill, Bull. Torrey bot. Club 32(9): 479, 1905 (=Polyporus scabrosus Pers., in Gaudichaud-Beaupré in Freycinet, Voy. Uranie., Bot.: 172, 1827).

Basidiome annual to biennial, resupinate, effuse-reflexed to pileate, pilear surface when present glabrous, white to cream, then with a reddish cuticle. Pore surface white to cork-coloured, pores elongated to sinuous, context white to cream. Hyphal system trimitic, generative hyphae with clamps, skeletal and binding hyphae hyaline. Cystidia none. Basidia clavate, with 4-stergimata, with a basal clamp. Basidiospores cylindrical to oblong ellipsoid, smooth, hyaline, negative in Melzer's reagent. Causing a white rot in hardwoods.

Remarks - Earliella seems close to Trametes Fr., sharing the trimitic hyphal system and type of rot, but differing in the resupinate basidiomes with sinuous pores.

Echinodontiellum Nakasone \& S.H. He, Mycologia 109 (4): 571, 2017.

Type species - Echinodontium japonicum Imazeki, Japanese Journal of Botany 11: 520, 1935.

Basidiome perennial, resupinate to slightly effused-reflexed, woody hard. Hymenophore odontioid, gray to olive gray. Aculei scattered to dense, conical, rigid, brittle. Margin determined, velvety, narrow. Subiculum cinnamon or olive gray to brownish gray, woody hard, darkening in $\mathrm{KOH}$. Hyphal system dimitic. Generative hyphae thin- to thick-walled, hyaline, nodose-septate, with scattered secondary simple septa. Skeletal hyphae thick-walled to subsolid, light brown. Cystidia numerous, clavate, hyaline to light brown, thick-walled, apically encrusted, blunt, embedded or slightly projected. Basidia clavate, hyaline, with 4 sterigmata and a basal clamp connection. Basidiospores ellipsoid, hyaline, thick-walled, echinulate, amyloid. Causing a white rot on living Quercus in Japan and China. Monotypic genus.

Remarks - Echinodontiellum present basidiomes that are effused to effused-reflexed, whereas they are effused-reflexed to pileate, rarely effused, in Echinodontium. Echinodontiellum has context cinnamon to olive-grey or brownish grey that darkens in $\mathrm{KOH}$. In comparison, the context in Echinodontium species are brick red or brownish orange that turns pale brown to brown in $\mathrm{KOH}$. There are no significant microscopic differences between Echinodontiellum and Echinodontium. Echinodontiellum occurs exclusively on Quercus, whereas Echinodontium s.str. prefers gymnosperms (Liu et al. 2017).

Echinodontium Ellis \& Everh., Bull. Torrey bot. Club 27(2): 49, 1900.

Type species - Fomes tinctorius Ellis \& Everh., Bull. Torrey bot. Club 22(7): 362, 1895.

Basidiome perennial, sessile, ungulate to conchate, or effuse-reflexed to entirely resupinate, context woody to leathery, brick red, olivaceous, or cream-colored, hymenophore distinctly aculeate to warted, sometimes almost smooth, inner tissue of teeth continuous with context. Hyphal system dimitic, with generative hyphae with clamps and secondary simple septa, thin-walled to solid and skeletal hyphae. Cystidia becoming thick-walled, dark red to hyaline, upper portion heavily encrusted, clavate to fusiform. Basidia clavate, with 4-sterigmate, and a basal clamp. Basidiospores hyaline, thick-walled, globose to ellipsoid, smooth to echinulate, strongly amyloid in Melzer's reagent.

Remarks - It is characterized by the perennial, usually pileate basidiomes with hydnoid hymenophore, dimitic hyphal system with encrusted skeletocystidia, and amyloid usually echinulate basidiospores. Echinodontium, with other corticioid and pileate fungi, frequently cluster 
together into a clade that has been circumscribed variously as the Bondarzewiaceae or the Echinodontiaceae in the Russulales (Larsson \& Larsson 2003, Miller et al. 2006). In the phylogenetic tree by Liu et al. (2017), Echinodontium, Amylostereum Boidin, Echinodontiellum S.H. He \& Nakasone, and Larssoniporia Y.C. Dai, Jia J. Chen \& B.K. Cui are included in the Echinodontiaceae, whereas Bondarzewia, Heterobasidion, Laurilia Pouzar, and Lauriliella Nakasone \& S.H. He, are in the Bondarzewiaceae; Perplexostereum Ryvarden \& Tutka forms a distinct clade from the Echinodontiaceae and Bondarzewiaceae.

Efibula Sheng H. Wu, Acta Bot. Fennica 142: 21, 1990.

Type species - Efibula tropica Sheng H. Wu, Acta Bot. Fennica 142: 25, 1990.

Basidiome effused, subceraceous or ceraceous, hymenial surface usually smooth. Hyphal system monomitic, hyphae simple-septate. Subiculum composed of a thin basal layer, and a prominent medullary layer with dense or compact texture. Cystidia usually lacking. Basidia clavate, 4-sterigmate. Basidiospores ellipsoid or narrowly ellipsoid, smooth, thin-walled, IKI-, CB-.

Remarks - Efibula has usually been included in Phlebia because of its dense contextual texture, or in Phanerochaete because of its simple-septate hyphae; but its relationship to the core of the two other genera seem to be distant. Efibula can be separated from Phlebia s.str. mainly by its simple-septate hyphae, but the separation from Phanerochaete requires more careful study. The medullary layer of Efibula is rather compact, all hyphae being interwoven and without a tendency to horizontal orientation. In Phanerochaete the subiculum usually has a somewhat loose texture, always having distinct spaces between the hyphae, which are usually straighter and horizontally oriented. Efibula was originally described to include two new species from Taiwan and some species formerly placed in Phlebia and Phanerochaete, characterized by simple-septate generative hyphae and dense contextual texture. Zmitrovich et al. (2006) expanded Efibula by transferring many acystidiate Phanerochaete species under this generic name. As it has been shown later (Wu et al. 2010) Efibula as seen by Zmitrovich et al. (2006) is polyphyletic.

Efibulella Zmitr., Folia Cryptogamica Petropolitana 6: 95, 2018.

Etymolgy - the generic name refers to another genus, Efibula Sheng H. Wu.

Type species - Grandinia deflectens P. Karst., Bidr. Känn. Finl. Nat. Folk 37: 239, 1882.

Basidiome of phlebioid habitus, annual, widely effused, ceraceous, hymenophore warted to smooth. Subiculum cornescent, hyaline. Hyphal system monomitic. Generative hyphae without clamp connections, hyaline, encrusted by resinous granules and deliquescent, CB-. Leptocystidia cylindrical, thin-walled. Basidia cylindrical, with central constriction, somewhat sinuous, 4-spored, simple-septate at the base. Basidiospores ellipsoid, thin-walled, IKI-, CB-. Causing a white rot.

Remarks - Efibulella mainly differs from Phlebia in the simple-septate hyphae, and from species of Phanerochaete by the ceraceous basidiomes, with hyphae usually embedded in a gelatinous matrix and difficult to discern (Zmitrovich 2018). Byssomerulius is also similar but differs by the byssoid basidiomes in contrasting to the ceraceous consistency of Efibulella. From Efibula, may be separated by the presence of leptocystidia.

Elaphocephala Pouzar, Česká Mykol. 37: 206, 1983.

Type species - Elaphocephala iocularis Pouzar, Česká Mykol. 37(4): 206, 1983.

Basidiome resupinate, effused, gelatinous, almost invisible, white to greyish. Hyphal system monomitic, generative hyphae with simple septa, thin-walled. Cystidia cylindrical with capitate apex, not encrusted. Basidia cylindrical, with 2-3 sterigmata, with a simple basal septum. Basidiospores lobed to forked, with two large outgrowths, smooth, thin-walled, IKI-.

Remarks - Elaphocephala is a monotypic genus characterized by the distinct antler-like basidiospores (Pouzar 1983). Its phylogenetic relationships remain still unclear (Larsson 2007b).

Elaphroporia Z.Q. Wu \& C.L. Zhao, MycoKeys 29: 88, 2018.

Type species - Elaphroporia ailaoshanensis Z.Q. Wu \& C.L. Zhao, MycoKeys 29: 89, 2018. 
Basidiomes annual, resupinate, becoming rigid and lightweight up on drying, pore surface cream to pale yellow when fresh, turning to yellow upon drying. Hyphal system monomitic, generative hyphae thick-walled bearing both clamp connections and simple septa, slightly amyloid, $\mathrm{CB}+$. Cystidia and cystidioles absent. Basidia clavate, with four sterigmata and a basal clamp connection. Basidiospores ellipsoid, hyaline, thin-walled, smooth, IKI-, CB-.

Remarks - According to Wu et al. (2018), phylogeny shows that the genus Elaphroporia belongs to the residual polyporoid clade in the family Meruliaceae. Elaphroporia is closely related to Flaviporus and Steccherinum. Morphologically, Elaphroporia resembles Ceriporia Donk and Phlebiporia Jia J. Chen, B.K. Cui \& Y.C. Dai. However, Ceriporia differs from Elaphroporia by the generative hyphae IKI-, CB-.

Emmia Zmitr., Spirin \& Malysheva, Mycena 6: 33, 2006.

Type species - Polyporus latemarginatus Durieu \& Mont., in Montagne, Syll. gen. sp. crypt. (Paris): 163, 1856.

Basidiome annual, resupinate to orbicular, soft and fragile; hymenophore bearing auxohymenium: tubular, one-layered, tubes firstly reticulate, later elongated, with thin dissepiments and large mouths, soft-waxy; trama subparallel; hyphal system monomitic; hyphae clampless, thinwalled in tubes, with thickened walls in subiculum, slightly amyloid; cystidia present as leptocystidia, thin-walled, cylindrical, naked or faintly encrusted; basidia clavate, four-spored, efibulate, with shallow medial constriction; basidiospores ellipsoid to short cylindrical, often abundant, cyanophilous, mitosporic state present in one species, of Sporotrichum type. Causing a white rot.

Remarks - Emmia latemarginata (Durieu \& Mont.) Zmitr., Spirin \& Malysheva has traditionally been classified in Oxyporus but it is strictly monomitic, while the other Oxyporus species have pseudodimitic structure consisting of more or less sklerified narrower hyphae. Hyphal structure brings Emmia latemarginata close to Ceriporia. According to molecular data, Emmia latemarginata is a member of phanerochaetoid clade and comes close to Ceriporia (Greslebin et al. 2004), i.e. it is rather distant from the other Oxyporus species.

Entomocorticium H.S. Whitney, Bandoni \& Oberw., Can. J. Bot. 65(1): 96, 1987.

Type species - Entomocorticium dendroctoni H.S. Whitney, in Whitney, Bandoni \& Oberwinkler, Can. J. Bot. 65(1): 96, 1987.

Remarks - Monotypic genus, not considered in this manual. The generic type grows in pupal chambers and larval galleries of Dendroctonus ponderosae (Curculionidae) on Pinus contorta var. latifolia (Whitney et al. 1987).

Eonema Redhead, Lücking \& Lawrey, Mycol. Res. 113(10): 1169, 2009.

Type species - Xenasma pyriforme M.P. Christ., Dansk bot. Ark. 19(no. 2): 108, 1960.

Basidiome resupinate, effused, pellicular, hymenial surface smooth, whitish. Hyphal system monomitic, hyphae with simple-septa. Cystidia absent. Basidia clavate to cylindrical, with 4sterigmata, and a simple basal septum. Basidiospores ellipsoid to pyriform, smooth, thin-walled, hyaline, IKI-.

Remarks - Eonema was proposed for Athelia pyriformis (M.P. Christ.) Jülich, a deviating species within Athelia living on ferns and grasses and phylogenetically related to Dictyonema but there is no obvious evidence of lichenization in specimens of $A$. pyriformis on fern fronds examined (Lawrey et al. 2009).

Epithele (Pat.) Pat., Essai Tax. Hyménomyc. (Lons-le-Saunier): 59, 1900.

Basionym - Hypochnus sect. Epithele Pat., N.T., Bull. Soc. Mycol. France 15: 202, 1899.

Type species - Athelia typhae Pers., Mycol. eur. (Erlanga) 1: 84, 1822.

Basidiome resupinate, adnate, at first orbicular, then becoming confluent, soft, white to pale yellowish white, hymenophore smooth to odontioid. Hyphal system monomitic, hyphae usually 
clamped, thin-walled, richly branched. Sterile hyphal pegs present. Cystidia lacking. Hyphidia present or lacking. Basidia clavate, sometimes slightly constricted. Basidiospores ellipsoid to fusiform, smooth to rough, thin- or thick-walled, non-amyloid, slightly stained in CB.

Remarks - Epithele is characterized by the odontioid hymenophore formed by sterile hyphal pegs and by the large fusiform basidiospores. It is phylogenetically included in the Polyporaceae. It presents a hymenial structure comparable to those present in Grammothele (Larsson 2007b). See Epithelopsis and Skeletohydnum for additional comments.

Epithelopsis Jülich, Persoonia 8(4): 457, 1976.

Type species - Epithele fulva G. Cunn., Trans. Roy. Soc. N.Z. 83: 631, 1956.

Basidiome annual, resupinate, effused, several cm large, adnate, consistency membranaceous, context homogeneous, hymenial surface pale coloured, odontioid, with sterile hyphal pegs composed of hyaline skeletal hyphae. Hyphal system dimitic, generative hyphae hyaline, cylindrical, loosely arranged, distinct, thick-walled (at least the basal ones), with clamps, skeletal hyphae hyaline, thick-walled. Gloeocystidia may be present. Basidia hyaline, clavate, 4-spored, with basal clamp. Basidiospores large, hyaline, somewhat thick-walled, strongly cyanophilous.

Remarks - Epithelopsis is characterized by the hyphal pegs composed by skeletal hyphae and by the thick-walled and cyanophilous basidiospores (Jülich 1976b). It differs from Epithele in the presence of thick-walled skeletal hyphae in the hyphal pegs, whereas Epithele lacks skeletals. Skeletohydnum is similar but it differs in the absence of cystidial elements, the presence of dendrohyphidia or paraphysoid hyphae, and in the thin-walled and acynophilous basidiospores.

Epulorhiza R.T. Moore, Mycotaxon 29: 94, 1987.

Type species - Rhizoctonia repens N. Bernard, Rev. gén. Bot. 9: 31, 1904.

Remarks - A name based on the anamorphic stage of Tulasnella J. Schröt.

Erastia Niemelä \& Kinnunen, Karstenia 45(2): 76, 2005.

Type species - Polyporus salmonicolor Berk. \& M.A. Curtis, Hooker's J. Bot. Kew Gard. Misc. 1: 104, 1849.

Basidiome annual, effused, poroid, soft, pale salmon colored, when dry discolored but not turning dark purple-red in bruised parts or elsewhere, $\mathrm{KOH}$ reaction almost null at pore surface, or light cherry red in ochraceous-colored mycelium of basidiocarp margins and subiculum. Hyphal system monomitic, hyphae thin-walled, with clamp connections, weakly amyloid in tube trama, acyanophilous. Brown oily matter abundant in between the hyphae. Cystidia none. Basidia clavate, with 4-sterigmate, with a basal clamp. Basidiospores thin-walled, ellipsoid, negative in both Melzer's reagent and Cotton Blue, with one prominent oil guttula.

Remarks - Ko et al. (2001) studied phylogenetic relationships among the Hapalopilus complex and they found that Hapalopilus salmonicolor is in fact alien to the genus, while $H$. rutilans and $H$. croceus are closely related. They proposed Hapalopilus salmonicolor to be included in the genus Sarcoporia. Niemelä et al (2005) found this proposal unacceptable and proposed the genus Erastia. The generic type of Erastia, Polyporus salmonicolor, is an American species, and in the sense given by European authors, the specimens previously identified as Hapalopilus salmonicolor sensu European authors, is heterogeneous and they belong to three different species: Aurantiporus priscus, E. aurantiaca, and E. ochraceolateritia. For further comments see Niemelä et al. (2012).

Erythricium J. Erikss. \& Hjortstam, Svensk bot. Tidskr. 64: 165, 1970.

Type species - Hyphoderma laetum P. Karst., Revue mycol., Toulouse 11(47): 206, 1889.

Basidiome resupinate, loosely adnate, hymenophore smooth, reddish, or pinkish red when fresh. Hyphal system monomitic, hyphae simple-septate, thin- to thick-walled in the subiculum, usually short-celled. Cystidia absent. Basidia clavate, constricted and sinuous, with 4-sterigmata, and a simple septum at the base. Basidiospores ellipsoid, smooth, thick-walled, IKI-, cyanophilous. 
Remarks - Erythricium is usually well recognized by the reddish colour of the basidiome; microscopically it reminds Phanerochate by the simple-septate hyphae, but differing in the shortcelled hyphae, constricted basidia and by the thick-walled and cyanophilous basidiospores (Eriksson \& Hjortstam 1970, Ghobad-Nejhad \& Hallenberg 2010). Phylogenetically it is classified close to Laetisaria in the Corticiaceae (Larsson 2007b).

Erythromyces Hjortstam \& Ryvarden, in Hjortstam \& Telleria, Mycotaxon 37: 55, 1990.

Type species - Hymenochaete crocicreas Berk. \& Broome, J. Linn. Soc., Bot. 14(no. 74): 68, 1873.

Basidiome resupinate, adnate, about 1-2 mm thick, hymenophore pilose by protruding cystidia, cinnamon brown to fawn-colored. Hyphal system monomitic, hyphae thin to moderately thick-walled or thick-walled, with clamps. Cystidia numerous, thick-walled, subulate, yellowish brown to reddish, ordinarily $100 \mu \mathrm{m}$ long. Basidia clavate, more or less pedunculate, with 4sterigmata and a basal clamp. Basidiospores ellipsoid to narrowly ellipsoid, thin-walled, smooth, IKI-.

Remarks - The noteworthy unique combination of a reddish and strongly pilose hymenophore and numerous, thick-walled encrusted cystidia characterize this genus. Other characteristics of importance are the monomitic hyphal system with clamped hyphae, hyaline and thin-walled spores and that the fungus produce a white pocket rot. Monotypic genus (Hjortstam \& Telleria 1990).

Etheirodon Banker, Bull. Torrey Bot. Club 29: 441, 1902.

Type species - Odontia fimbriata Pers., Observ. mycol. (Lipsiae) 1: 88, 1796.

Remarks - Proposed as a substitute for Odontia Fr. and a synonym of Steccherinum S.F. Gray.

Fasciodontia Yurchenko \& Riebesehl, Mycol. Progress 19: 178, 2020.

Type species - Odontia bugellensis Ces. in Rabenhorst, Klotzschii Herb.Viv.Mycol. no. 1915, 1855.

Basidiome resupinate, effused, membranaceous, cracking with age, margin thinning out, rarely abrupt. Hymenial surface minutely odontioid, whitish or cream-colored; aculei sterile except bases and consisting of projecting hyphae. Hyphal system pseudodimitic, hyphae clamped at all primary septa, colorless in water, slightly yellowish in mass in $\mathrm{KOH}$, moderately cyanophilous, negative in Melzer's reagent; skeletal-like thick-walled hyphae present in basal subhymenium and in aculeal trama; skeletal-like hyphae in aculei loosely encrusted, apically thin-walled, partly agglutinated with age. Cystidia from cylindrical to slightly moniliform and capitate. Basidia utriform to subcylindrical, with two slight constrictions, thin-walled or slightly thick-walled in lower part, with four-sterigmata, basally clamped. Basidiospores ellipsoid, smooth, with thickened or thick walls (about $0.2-0.3 \mu \mathrm{m}$ thick), colorless, IKI-, slightly to moderately cyanophilous.

Remarks - The main diagnostic features of the genus are minutely odontioid hymenophore, presence of thick-walled skeletal-like or sclerified hyphae, associated with aculeal trama, submoniliform cystidia, which are from seldom to numerous, confined mostly to the base of aculei, and thick-walled basidiospores. Xylodon is the closest genus to Fasciodontia in both morphological and molecular aspects (Yurchenko et al. 2020).

Fibriciellum J. Erikss. \& Ryvarden

Type species - Fibriciellum silvae-ryae J. Erikss. \& Ryvarden, Cortic. N. Eur., 3: 375, 1975.

Remarks - A synonym of Trechispora P. Karst.

Fibricium J. Erikss., Symb. bot. upsal. 16(1): 112, 1958.

Type species - Corticium rude P. Karst., Bidr. Känn. Finl. Nat. Folk 37: 143, 1882. 
Basidiome resupinate, effused, hymenophore smooth, margin rhizomorphic. Hyphal system dimitic, generative hyphae clamped, skeletal hyphae thick-walled, acyanophilous. Cystidia usually present, encrusted or smooth. Basidia clavate, with 4-sterigmata and a basal clamp. Basidiospores ellipsoid, smooth, thin-walled, IKI-, CB-.

Remarks - Fibricium is characterized by the dimitic hyphal system, the usual presence of cystidia and by the small thin-walled basidiospores. According to molecular data, it is polyphyletic and its relationships with other genera are still unclear. The type species, Fibricium rude belongs to the Hymenochaetales (Larsson et al. 2006, Larsson 2007b).

Fibrodontia Parmasto, Consp. System. Corticiac. (Tartu): 174, 1968.

Type species - Fibrodontia gossypina Parmasto, Consp. System. Corticiac. (Tartu): 207, 1968 (=Cystidiodendron fimbriatum Rick, Lilloa 9: 218, 1943).

Basidiome resupinate, easily detached in pieces, effused, adnate, hymenophore odontioid with cylindrical aculei. Hyphal system pseudodimitic, all hyphae with clamps, pseudoskeletal hyphae thick-walled. Cystidia absent. Basidia clavate to suburniform, with 4-sterigmata and a basal clamp. Basidiospores ellipsoid, smooth, thin-walled, hyaline, IKI-, CB-.

Remarks - Fibrodontia is characterized by the odontioid hymenophore, and dimitic or pseudodimitic hyphal system. Fibrodontia gossypina belongs to the trechisporoid clade close to other Trechispora species. Although morphologically it looks similar to Hyphodontia species, phylogenetically it seems not to be very closely related (Binder et al. 2005, Larsson 2007b). Baltazar et al. (2016) checked Cystidiodendron fimbriatum Rick and concluded it is conspecific with Fibrodontia gossypina Parmasto. See also comments under Cystidiodendron Rick.

Fibroporia Parmasto, Consp. System. Corticiac. (Tartu): 176, 1968.

Type species - Boletus vaillantii DC., in De Candolle \& Lamarck, Fl. franç., Edn 3 (Paris) 5/6: 38, 1815.

Basidiome annual, resupinate, often widely effused, soft-fibrous, margin often wide with rhizomorphs. Pores circular to angular, subiculum soft and cottony. Hyphal system dimitic, generative hyphae with clamps, hyaline, thin-walled, skeletal hyphae predominant in context and rhizomorphs, solid to thick-walled, hyaline, non-septate, straight, and unbranched, rarely dichotomously branched, negative in Melzer's reagent. Cystidia none, fusoid cystidiols may be present. Basidia clavate, 4-sterigmate, with a basal clamp. Basidiospores ellipsoid, hyaline, smooth, negative in Melzer's reagent. Causing a brown cubical rot.

Remarks - The genus is morphologically similar to Antrodia, but the presence of a rhizomorphic margin separate both genera. Phylogenetic analysis supports this separation (Bernicchia et al. 2012, Bernicchia \& Gorjón 2020, Rajchenberg et al. 2011).

Fibulomyces Jülich, Willdenowia Beih. 7: 178, 1972.

Type species - Corticium mutabile Bres., Fung. Trident. 2(11-13): 59, 1898.

Remarks - A synonym of Leptosporomyces Jülich.

Fibuloporia Bondartsev \& Singer ex Singer, Mycologia 36: 67, 1944.

Type species - Boletus molluscus Pers., Syn. meth. fung. (Göttingen) 2: 547, 1801.

Remarks - A synonym of Trechispora P. Karst.

Flavidoporia Audet, Mushrooms nomenclatural novelties 4: 1, 2017.

Type species - Poria pulvinascens Pilát, Sb. Nár. Mus. v Praze, Rada B, Prír. Vedy 9(2): 106, 1953.

Remarks - According to Audet (2017d), Flavidoporia differs from Antrodia s.str. by basidiomata annual to perennial, resupinate to pseudopileate, without context, yellowish pores, smaller basidia, absence of hyphidia or false skeletocystidia, and smaller oblong to ellipsoid basidiospores. 
Flaviporus Murrill, Bull. Torrey bot. Club 32(7): 360, 1905.

Type species - Polyporus rufoflavus Berk. \& M.A. Curtis, Bot. J. Linn. Soc. 10: 310, 1869 (=Boletus brownii Humb. [as 'browni'], Fl. Friberg. Spec. (Berlin): 101, 1793)

Basidiome effused reflexed to resupinate, annual; pileus when present dark brown to bay, glabrous. Pore surface vivid chrome yellow, pores tiny, almost invisible to the naked eye, tubes concolorous; context thin and yellow. Hyphal system dimitic, generative hyphae with clamps, skeletal hyphae abundant. Skeletocystidia, with densely encrusted apex. Basidia clavate, with 4sterigmate, with a basal clamp. Basidiospores ellipsoid, smooth, negative in Melzer's reagent, shorter than $5 \mu \mathrm{m}$. Causing a white rot.

Remarks - The genus is morphologically related to poroid species of Steccherinum, separated only by the vivid yellow pore surface (Bernicchia \& Gorjón 2020).

Flavodon Ryvarden, Norwegian J. Botany, 20: 3, 1973.

Type species - Irpex flavus Klotzsch, Linnaea 8: 488, 1833.

Basidiome annual, resupinate to pileate, reddish to brown with $\mathrm{KOH}$, pileus adpressed tomentose, yellowish to ochraceous-grey, hymenophore first poroid, then hydnoid to irpicoid, context bright sulphurous yellow (colour fades to cinnamon or ochraceous in old or herbarium specimens). Hyphal system dimitic, generative hyphae with simple septa, skeletal hyphae thickwalled and partly bent into the hymenium as smooth or encrusted cystidia, spores broadly ellipsoid, smooth, hyaline and non-amyloid. On deciduous wood, Monotypic genus.

Remarks - Often considered a synonym of Irpex Fr. but separated by some authors by its yellowish color and the reddish reaction with KOH. According to Ryvarden (1973) it must be regarded as a tropical counterpart to the temperate-boreal genus Irpex s.str.

Flavophlebia (Parmasto) K.H. Larss. \& Hjortstam, Mycotaxon 5: 475, 1977.

Basionym - Phlebia Fr. subgen. Lilaceophlebia Parmasto sectio Flavophlebia Parmasto, Consp. System. Corticiac. (Tartu): 94, 1968.

Type species - Corticium sulphureoisabellinum Litsch., Sb. nár. Muz. Praze, B 2(3): 43, 1940.

Basidiome resupinate, effused, at first thin, thickening with age, ceraceous, continuous, cracking with age, hymenophore smooth, margin indistinctly thinning out. Hyphal system monomitic, hyphae with clamps, mostly indistinct, thin-walled. Cystidia cylindrical to subfusiform, thin-walled, usually enclosed. Basidia terminal, slightly stalked, with a basal clamp, usually with 4sterigmata. Basidiospores oblong, subglobose or globose with sublateral apiculus, smooth, IKI-, CB-.

Remarks - Flavophlebia is easily recognized by its yellowish basidiome, clavate basidia, and by its subglobose, oil rich basidiospores with distinct, sublateral apiculus. Spore morphology reminds those of Radulomyces confluens, even if they are not closely related (Hjortstam \& Larsson 1977). Phylogenetic relationships of Flavophlebia are still unclear (Larsson 2007b).

Fragiliporia Y.C. Dai, B.K. Cui \& C.L. Zhao, Fungal Diversity 70: 121, 2015.

Type species - Fragiliporia fragilis Y.C. Dai, B.K. Cui \& C.L. Zhao, Fungal Diversity 70: 121, 2015.

Basidiome resupinate, very soft when fresh, becoming brittle when dry (become almost powdery when bruised). Hymenophore poroid, pore surface greyish-buff to lavender when fresh, turning violaceous grey to greyish brown upon drying, margin fimbriate. Subiculum orange yellow. Hyphal system monomitic, generative hyphae with clamps, thick-walled, hyphae frequently branched from clamps connections, hyphae encrusted with crystals. Cystidia absent. Basidia clavate to pyriform, with 4-sterigmata and a basal clamp. Basidiospores allantoid, smooth, thin-walled, IKI-, CB-. Causing a white rot.

Remarks - Fragiliporia according to authors forms an independent family, Fragiliporiaceae Y.C. Dai, B.K. Cui \& C.L. Zhao, in a monophyletic group within Polyporales. Particular characters 
are resupinate basidiomes with very soft tubes when fresh, which become brittle when dry (becoming almost powdery when bruised), a monomitic hyphal system with thick-walled generative hyphae, clamp connections, and frequently $\mathrm{H}-$, $\mathrm{W}$ - or $\mathrm{Y}$-shaped hyphae branching from the clamp connections (Zhao et al. 2015). It seems macro and micro morphologically related to Bjerkandera because the violaceous to greyish-black poroid surface and the thick-walled generative hyphae.

Frantisekia Spirin \& Zmitr., Czech Mycology 59 (2): 142, 2007.

Type species - Poria fissiliformis Pilát, Studia bot. cechosl.: 1, 1940.

Basidiome annual, pileate, effused-reflexed or resupinate, cream to pale ochraceous, and fleshy in fresh condition, often darkening and fragile when dry. Hymenophore poroid, tubes strongly agglutinated in herbarium specimens; pores small, 6-10 per mm. Hyphal structure pseudodimitic; generative hyphae clamped, hyaline or yellowish, thin- to somewhat thick-walled, pseudoskeletal hyphae present in context and (or) tubes, thick-walled, with rare clamps and septa, even or inflated, often weakly cyanophilous. No cystidia; cystidioles sometimes present. Basidia narrowly clavate, four-spored, clamped; basidiospores thin-walled, oblong ellipsoid to cylindrical, negative in both Melzer's reagent and Cotton Blue. Causing a white rot in dead hardwoods.

Remarks - The best diagnostic characters of Frantisekia are the pseudodimitic hyphal structure, narrowly clavate basidia, and small spores. Relatives of Frantisekia might be found among Ceriporiopsis species, with which it shares fleshy resupinate basidiomes, agglutinated tubes, the predominance of thin-walled generative hyphae and the type of rot. However, the genus Ceriporiopsis is evidently heterogeneous (Zmitrovich et al. 2006; Tomšovský et al. 2010) and needs to be split into small homogeneous genera (Spirin \& Zmitrovich 2007).

Funalia Pat., Essai Tax. Hyménomyc. (Lons-le-Saunier): 95, 1900.

Type species - Polyporus mons-veneris Jungh., Verh. Batav. Genootsch. Kunst. Wet. 17(2): 61, 1838; =Polyporus leoninus Klotzsch, in Dietrich, Fl. Regn. Boruss. 7: 459, 1839.

Basidiome annual, pileate and broadly attached, mostly single, semicircular to elongated, pileus densely hirsute, pure white when fresh, straw-colored when dry or old, pore surface concolorous with pileus, pores angular, 1-2 per mm or larger in old specimens, with age strongly lacerate in the dissepiments, context dense and brown to dark brown. Hyphal system trimitic, generative hyphae with clamps, hyaline skeletal and binding hyphae, cystidia none, spores cylindrical, smooth and hyaline 10-14 $\mu$ m long. On deciduous wood.

Remarks - According to the molecular phylogeny of Trametes by Justo \& Hibbet (2011), the genera Artolenzites Falck, Coriolopsis Murrill (the generic type), Coriolus Quél., Cubamyces Murrill, Cyclomycetella Murrill, Lenzites Fr., Poronidulus Murrill, Pseudotrametes Bondartsev \& Singer, and Pycnoporus P. Karst. are considered synonyms of Trametes, recommending the use of a single generic name Trametes for the trametoid clade as the best taxonomic and nomenclatural option to avoid many nomenclatural changes against the alternative scenarios dividing the trametoid clade in five or ten different genera. Out of the trametoid clade is recovered Trametopsis cervina (Schwein.) Tomšovský in the phlebioid clade and Coriolopsis trogii (Berk.) Domański outside the trametoid clade, closely related to Coriolopsis gallica (Fr.) Ryvarden. The name Funalia Pat. could be adopted for the C. gallica group, but no molecular data are available for the type species of Funalia, viz. Polyporus mons-veneris Jungh. Morphological characters of Polyporus mons-veneris, especially the spore length above $10 \mu \mathrm{m}$ and the strigose suggest a relationship with the $C$. gallica group (Justo \& Hibbet 2011). Funalia can be separated in morphology from Trametes by dark context composed of coloured vegetative hyphae, the dense and thick mat of strigose hairs on the pileus and the lacerate pore mouths with age.

Galzinia Bourdot, Compt. Rend. Assoc. Franç. Avancem. Sci. 45: 577, 1922.

Type species - Galzinia pedicellata Bourdot, Compt. Rend. Assoc. Franç. Avancem. Sci. 45: 577, 1922. 
Basidiome resupinate, adnate, effused, membranaceous to ceraceous, pruinose when dry, hymenophore smooth, margin indeterminate. Hyphal system monomitic, hyphae with clamps, thinto thick-walled. Cystidia (leptocystidia) present or lacking. Basidia cylindrical to urniform, with 4sterigmata and with a basal clamp, new basidia are occasionally produced by internal repetition. Basidiospores ellipsoid, cylindrical to allantoid, smooth, thin-walled, non-amyloid.

Remarks - Galzinia is morphologically defined by the curved basidiospores and by the urniform basidia with 4-sterigmata. Athelopsis differs by the pedunculate basidia and usually smaller basidiospores and Sistotrema by different spore morphology and hyphae usually with many oily contents. Phylogenetically Galzinia is included in the Corticiaceae (Larsson 2007b).

Galziniella Parmasto, Consp. System. Corticiac. (Tartu): 39, 1968.

Type species - Galziniella pereximia Parmasto, Consp. System. Corticiac. (Tartu): 209, 1968. (=Gloeocystidium sernanderi Litsch., Svensk bot. Tidskr. 25(3): 437, 1931).

Remarks - A synonym of Sistotrema Fr.

Geesterania Westph., Tomšovský \& Rajchenb., Persoonia 41: 134, 2018.

Type species - Poria carneola Bres., Hedwigia 35: 282, 1896.

Basidiome resupinate, adnate, becoming reddish when bruised and/or upon drying, soft to fleshy when fresh, becoming harder and somewhat waxy when dried. Hymenophore poroid; pores regular to irregular, round to angular. Hyphal system dimitic, generative hyphae clamped, skeletal hyphae metachromatic, hyphae IKI-. Skeletocystidia present in the trama and dissepiments, cylindrical to clavate, incrusted at the apex with thin crystals, usually very long and appearing as skeletal hyphae ends, metachromatic, IKI-. Basidiospores ellipsoid to sub-cylindrical, hyaline, smooth, IKI-.

Remarks - Geesterania is characterized by resupinate, somewhat fleshy basidiomes, typically becoming reddish when bruised or dried. The color change is variable, and in some basidiomes only some reddish spots appear while others become pale reddish-brown to flesh colored throughout. Microscopically, the very long finely incrusted (skeleto) cystidia are typical. In the trama, the cystidia are usually markedly thick-walled and long, arising from skeletal hyphae that slowly increase in diameter. For that reason, the distinction between skeletal hyphae and cystidia sometimes is not clear and the cystidia may be interpreted as wide incrusted skeletal hyphae ends. In addition, the skeletal hyphae and cystidia are metachromatic, while the generative hyphae, hymenium and basidiospores are non-metachromatic in Cresyl Violet. Geesterania differs from other polypore genera exactly by this combination of characteristics, which is unique and unknown in other poroid fungi. Phylogenetically, the closest related polypore species known is Phlebioporia bubalina (Chen \& Cui 2013) but, morphologically, it substantially differs by presenting a monomitic hyphal system with simple septate dextrinoid hyphae and lack of cystidia. The recently described genus and species Luteoporia albomarginata (Wu et al. 2016) is also phylogenetically related to Geesterania, presenting similar resupinate basidiomes with the presence of cystidia-like structures. However, Luteoporia differs in being monomitic, turning red in contact with $\mathrm{KOH}$, and having differently shaped thin-walled cystidia (Westphalen et al. 2018).

\section{Gelatoporia Niemelä, Karstenia 25(1): 22, 1985}

Type species - Poria subvermispora Pilát, Stud. Bot. Čechoslov. 3: 2, 1940.

Basidiome annual, resupinate, light-colored; tubes soft and hygrophanous when fresh. Hyphal system monomitic; hyphae with thin or slightly and evenly thickened walls but lacking solid side branches or other differentiated hyphal types, hyaline, with clamp connections, CRB+, glued together into a gelatinized structure in dry tube dissepiments, adjacent subiculum or both; dissepiment structure often layered with special medullary hyphae; hymenial cystidia absent, but bottle-shaped cystidioles some-times present; spores cylindrical, IKI-, CB-. Causing white rot on both coniferous and deciduous trees. 
Remarks - Gelatoporia includes resupinate polypores with a monomitic hyphal structure causing a white rot. Hyphae are generally glued together into a gelatinized matrix in a dry tube dissepiment and or in the subiculum. Gelatoporia is phylogenetically related to Obba and Cinereomyces, see Miettienen \& Rajchenberg (2012).

Ginnsia Sheng H. Wu \& Hallenb., in Wu, Nilsson, Chen, Yu \& Hallenberg, Fungal Diversity 42(1): 114, 2010.

Type species - Thelephora viticola Schwein.: Fr., Schrift. Naturforsch. Gesellsch. (Leipzig) 1: 107.1822

Basidiome resupinate, pellicular-membranaceous. Hymenial surface smooth. Hyphal system monomitic, hyphae simple-septate. Cystidia present. Basidia clavate with stalked bases, 4sterigmate, and guttulate. Basidiospores ellipsoid, smooth, thin-walled, guttulate, inamyloid, nondextrinoid

Remarks - Ginnsia was erected to accommodate Thelephora viticola, a deviating species formerly classified in Phanerochaete. Ginnsia has guttulate basidia with stalked bases, big and usually bearing one prominent oil-drop basidiospores, and fairly narrow subicular hyphae chiefly vertical. In addition to these unusual characters within Phanerochaete, Thelephora viticola is phylogenetically assigned to the Hymenochaetales (Wu et al. 2010).

Globuliciopsis Hjortstam \& Ryvarden, Synopsis Fungorum 18: 22, 2004. 2004.

Type species - Globuliciopsis fuegiana Hjortstam \& Ryvarden, Synopsis Fungorum 18: 24,

Basidiome resupinate, fairly thick, closely adnate, slightly stratified, pale brown, hymenophore smooth, subiculum compact, brownish. Hyphal system monomitic, subicular hyphae thin or moderately thick-walled, short-celled, other hyphae thin-walled, straight, hyphae hyaline, originally erroneously described without clamps but the original draw of the type species showing clamps. Cystidia absent, but with hyphal ends and dendrohyphidia. Basidia terminal, arranged in a relatively loose palisade, up to $100 \mu \mathrm{m}$ long with two or sometimes three to four sterigmata. Basidiospores almost globose, smooth, hyaline, with a slight wall thickening, inamyloid, indextrinoid, slightly cyanophilous.

Remarks - Globuliciopsis was originally described with a monomitic hyphal system and erroneously without clamps, even if the type species, G. fuegiana has clamp connections as it was showed in the original drawing (Hjortstam \& Ryvarden 2004c). Later, Globuliciopsis lindbladii Hjortstam \& Ryvarden, was added to Globuliciopsis (Hjortstam \& Ryvarden 2007); this species is superficially similar to $G$. fuegiana but differing in a di- trimitic hyphal system, generative hyphae lacking clamp connections, and possessing SA- gloeocystidia. According to Karl-Henrik Larsson who examined the type, G. lindbladii is conspecific to Licrostoma subgiganteum (comm. pers.).

Globulicium Hjortstam, Svensk bot. Tidskr. 67: 108, 1973.

Type species - Corticium hiemale Laurila, Ann. bot. Soc. Zool.-Bot. fenn. Vanamo 10(4): 4, 1939.

Basidiome resupinate, adnate, at first orbicular, then effused, ceraceous, hymenophore smooth, margin indeterminate. Hyphal system monomitic, generative hyphae with clamps, thinwalled, and richly branched. Cystidia absent, paraphysoid hyphae present, some encrusted. Basidia clavate to cylindrical, constricted, with 4-sterigmata, and a basal clamp. Basidiospores globose, smooth, thin-walled, IKI-.

Remarks - Globulicium reminds in some respects Hyphoderma, differing by the globose basidiospores and the encrusted paraphysoid hyphae, and Radulomyces, but basidiospores in Globulicium are considerably larger and thin-walled. Phylogenetic relationships of Globulicium are unclear, it belongs to the Rickenella family that includes a mixture of basidiome morphologies (Larsson 2007b). 
Gloeocorticium Hjortstam \& Ryvarden, Mycotaxon 25: 551, 1986. 1986.

Type species - Gloeocorticium cinerascens Hjortstam \& Ryvarden, Mycotaxon 25: 551,

Basidiome resupinate, effuse, adnate, hymenophore odontioid. Hyphal system monomitic to dimitic with thick-walled long-celled hyphae in the core of the aculei, hyphae with clamps, IKI-. Gloeocystidia moniliform, thin-walled, distinctly amyloid. Basidia clavate, with 4-sterigmata, and a basal clamp. Basidiospores subglobose, smooth, thick-walled, IKI-, CB-, with a distinct apiculus placed laterally.

Remarks - Gloeocorticium is characterized by the moniliform and amyloid gloeocystidia and the thick-walled with an apiculus laterally placed. According to Hjortstam \& Ryvarden (1986) it has similar characters to those of Radulomyces by the spores with a lateral apiculus and basidia. It was created for a species from North Argentina (Iguazu).

Gloeocystidiellum Donk, Medded. Nedl. Mycol. Ver. 18-20: 156, 1931, em. Donk, Fungus 26: 8, 1956.

Type species - Corticium porosum Berk. \& M.A. Curtis, Ann. Mag. nat. Hist., Ser. 5 3(15): 211, 1879.

Basidiome resupinate, effuse, membranaceous or ceraceous, hymenophore smooth, rarely grandinioid or odontioid. Hyphal system monomitic, hyphae with clamps or simple-septate. Gloeocystidia present, usually SA+. Basidia clavate, with 4-sterigmata. Basidiospores suballantoid, ellipsoid, subglobose or globose, verrucose or aculeate (seemingly smooth in $\mathrm{KOH}$ ), thin-walled, amyloid, CB-.

Remarks - Gloeocystidiellum was proposed by Donk (1931) for the misapplied Gloeocystidium P. Karst., afterwards emended to include monomitic species, with euhymenium, gloeocystidia and amyloid basidiospores (Donk 1956). The genus as above defined was regarded as heterogeneous, but useful for delimit a wide number of species. Eriksson \& Ryvarden (1975) subdivided Gloeocystidiellum into seven groups on the basis of basidiome texture, hyphal septation, cyanophily of hyphae, sulfoaldehyde reaction of gloeocystidia, basidiospores shape and nature of the spore wall. At the present time Gloeocystidiellum s.l. and the seven groups proposed by Eriksson \& Ryvarden (1975) are reorganized as follow: Boidinia (G. furfuraceum group), Conferticium (G. ochraceum group), Gloeocystidiellum (G. porosum group), Gloeopeniophorella (G. convolvens group), Gloiothele (G. lactescens group), Megalocystidium (G. luridum group), Vesiculomyces (G. citrinum group). In addition, Wu $(1995,1996)$ described other closely related genera: Amylofungus with amyloid hyphae, gloeocystidia, basidia and basidiospores; Dextrinocystidium with dextrinoid lamprocystidia (synonym of Gloeopeniophorella according to Hjortstam \& Ryvarden 2007); and Gloeomyces, with a distinct dimitic hyphal system and with brownish binding hyphae. For further comments and keys to resupinate corticioids with gloeocystidia and amyloid basidiospores see Wu l.c. and Boidin et al. (1997a).

Gloeocystidiopsis Jülich, Int. J. Mycol. Lichenol. 1: 27, 1982.

Type species - Gloeocystidiellum flammeum Boidin, Cahiers de La Maboké 4(3): 7, 1966.

Basidiome resupinate, effuse, membranaceous to subgelatinous, hymenophore smooth, pale to orange coloured. Hyphal system monomitic, hyphae with simple-septate. Gloeocystidia present, $\mathrm{SA}+$. Basidia clavate, with 4-sterigmata. Basidiospores ellipsoid, verrucose, thin-walled, strongly amyloid, CB-.

Remarks - It differs morphologically from Gloeocystidiellum Donk in the simple-septate hyphae and by the holocoenocytic mycelium. binucleate in Gloeocystidiellum (Jülich 1982). The separation is phylogenetically supported (Larsson \& Larsson 2003).

Gloeocystidium P. Karst., Bidr. Känn. Finl. Nat. Folk 48: 429, 1889.

Type species - Grandinia exsudans P. Karst., Meddn Soc. Fauna Flora fenn. 9: 51, 1882.

Remarks - A synonym of Dacryobolus Fr. 
Gloeodontia Boidin, Cahiers de La Maboké 4: 22, 1966.

Type species - Irpex discolor Berk. \& M.A. Curtis, Grevillea 1(3): 45, 1872.

Basidiome resupinate, effused, adnate, hymenophore hydnoid, subiculum well developed. Hyphal system monomitic or dimitic, generative hyphae with clamps, thin-walled or with slight thickened walls, skeletal hyphae rarely branched, aseptate or with rare septa, thick-walled. Cystidia (pseudocystidia) arising in tooth trama, cylindrical, heavily encrusted with hyaline crystals. Gloeocystidia scattered in tooth trama, frequent in hymenium, blue to blue-black in sulfuric benzaldehyde. Halocystidia present in one species. Basidia cylindrical to urniform, with 4sterigmata, with a basal clamp. Basidiospores hyaline to pale yellow under the microscope, thickwalled, asperulate, amyloid.

Remarks - Gloeodontia is morphologically defined by the hydnoid hymenophore, gloeocystidia, encrusted pseudocystidia, and by the amyloid asperulate basidiospores. Gloeodontia forms a well-defined group within the russuloid lineage (Larsson 2007b).

Gloeohypochnicium (Parmasto) Hjortstam, Mycotaxon 28: 30, 1987.

Basionym - Hypochnicium subgen. Gloeohypochnicium Parmasto, Consp. System. Corticiac. (Tartu): 116, 1968.

Type species - Gloeocystidium analogum Bourdot \& Galzin, Bull. Soc. mycol. Fr. 28(4): 36, 1913.

Basidiome resupinate, adnate, effused, hymenophore smooth to tuberculate, margin indeterminate. Hyphal system monomitic, hyphae with clamps, thin- to thick-walled. Gloeocystidia present, SA+. Basidia cylindrical to narrowly clavate, usually constricted, with a basal clamp, with 4-sterigmata. Basidiospores ellipsoid to globose, distinctly thick-walled, warted, IKI-, cyanophilous.

Remarks - Gloeohypochnicium is separated from Hypochnicium on the basis of the sulfopositive gloeocystidia, the ornamentation of basidiospores, and the swelling of the spore wall (Hjortstam 1987b). Phylogenetically the relationships of Gloeohypochnicium remain still unclear (Larsson 2007b).

Gloeomyces Sheng H. Wu, Mycotaxon 58: 47, 1996.

Type species - Gloeomyces graminicola Sheng H. Wu, Mycotaxon 58: 49, 1996.

Basidiome resupinate, effuse, membranous. Hyphal system dimitic, generative hyphae simple-septate, binding hyphae sparsely distributed in subiculum, yellow or brown. Gloeocystidia numerous, moniliform, slightly SA+. Basidia utriform, with 4-sterigmata, with a simple basal septum. Basidiospores ellipsoid, smooth, thin-walled, amyloid, CB-.

Remarks - Gloeomyces is characterized among Gloeocystidiellum s.l. by the dimitic hyphal system with binding hyphae in the subiculum (Wu 1996).

Gloeopeniophora Höhn. \& Litsch., Sber. Akad. Wiss. Wien, Math.-naturw. Kl., Abt. 1 116: 815, 1907.

Type species - Thelephora incarnata Pers., Syn. meth. fung. (Göttingen) 2: 573, 1801.

Remarks - A synonym of Peniophora Cooke.

Gloeopeniophorella Rick, Brotéria, sér. Ci. Nat. 3: 47, 173, 1934.

Type species - Gloeopeniophorella rubroflava Rick, Brotéria, sér. Ci. Nat. 3: 47, 1934.

Basidiome resupinate, effused, adnate. Hymenophore almost smooth to slightly tuberculate, usually ochraceous. Hyphal system monomitic, hyphae hyaline, thin-walled or with a slight thickened wall, simple-septate. Cystidia of two kind: 1) lamprocystidia numerous, hyaline, moderately thick-walled, without dextrinoid reaction (see also Dextrinocystidium for a similar genus with dextrinoid lamprocystidia), 2) gloeocystidia few to numerous, thin-walled, with or without positive sulphovanillin reaction. Basidia narrowly clavate, with 4-sterigmata, without a 
basal clamp-connection. Basidiospores more or less ellipsoid, distinctly rugose in Melzer's reagent, seemingly smooth in $\mathrm{KOH}$, amyloid.

Remarks - Gloeopeniophorella is characterized among the Gloeocystidiellum s.l. by the presence of lamprocystidia. Dextrinocystidium is a similar genus with dextrinoid lamprocystidia, and a synonym according to Hjortstam \& Ryvarden (2007). It belongs to the Russulaceae, closely related to Boidinia (Larsson 2007b).

Gloeophyllum P. Karst., Bidr. Känn. Finl. Nat. Folk 37: 79, 1882.

Type species - Agaricus sepiarius Wulfen, in Jacquin, Collnea bot. 1(2): 339, 1787.

Basidiome annual to perennial, sessile, pileate, dimidiate to broadly attached or rosette shaped, brown, pileus smooth, tomentose to hispid, hymenophore irregular, distinctly lamellate to daedaleoid, golden to deep brown, hyphal system di- or trimitic, generative hyphae with clamps, skeletal hyphae golden-brown, dominating in the fruitbodies, binding hyphae rare to common, cystidia mostly present, thick to thin-walled, smooth or with a slight apical encrustation, basidiospores cylindrical, smooth, hyaline and non-amyloid. Causing a brown rot.

Remarks - Gloeophyllum is closely related to Funalia, sharing with this genus the coloured skeletal hyphae, cylindrical, smooth, non-amyloid and moderately large spores and clamped generative hyphae. The catahymenium found in Gloeophyllum is however, absent in most Funalia species which also have a poroid hymenophore. Recently, He et al. (2014) proposed a phylogeny supporting the separation of Gloeophyllum s.l. in smaller entities, establishing differences between Gloeophyllum s.str., and Griseoporia Ginns (with typically poroid hymenophore and resupinate to effuse-reflexed basidiomes), Osmoporus Singer (poroid hymenophore and pileate basidiomes), and Hispidaedalea Y.C. Dai \& S.H. He (daedaleoid to lamellate hymenophore, pileate basidiomes, and trimitic hyphal system).

Gloeoporellus Zmitr., Folia Cryptogamica Petropolitana 6: 85, 2018.

Type species - Polyporus merulinus Berk., in Hooker, Bot. Antarct. Voy., III, Fl. Tasman. 2: 254, 1860.

Basidiome resupinate, of tyromycetoid to ceriporioid habitus with narrow matted pilei or totally prostrate and apricot to carmine red coloration; hymenophore poroid with 2-3-layered elongated pores, cornescent, carmine red. Context apricot, ceraceous. Hyphal system dimitic. Generative hyphae clamped, hyaline. Skeleto-binding hyphae regularly branched, thick-walled, pigmented, $\mathrm{CB}+$. Binding hyphae that in the context are dicophyses-like to arboriform or arachnoid in aspect; these dicophysal elements are present in the dissepiments and the tubes only when the hymenium becomes old. All the basidiome filled with an oily-like resinous substance responsible for the basidiome coloration. Cystidia none, but some cystidiols may be present. Basidia shortclavate, 4-spored with a basal clamp. Basidiospores cylindrical to allantoid, smooth, thin-walled, IKI-, CB-. Causing a white rot.

Remarks - Polyporus merulinus has an austral distribution (Australia, New Zealand, Tasmania, and South Argentina and Chile). There is a good description and drawing in Rajchenberg (2006). He considers the species under Ceriporiopsis but noted the unique features among polypore fungi, highlighting the particular dicophysal elements and the oily resinous substances that makes this species very particular. According to Zmitrovich (2018), the closely related genus is Skeletocutis, but this genus differs from Gloeoporellus by uncolored context and less branched skeletals.

Gloeoporus Mont., Ann. Sci. Nat. Bot. Ser. 2, 17: 126, 1842.

Type species - Gloeoporus conchoides Mont., Ann. Sci. Nat. Bot. Ser. 2, 17: 126, 1842.

Basidiome annual, resupinate to effuse-reflexed, abhymenial surface tomentose, hymenial surface poroid, rose to orange-reddish, pores round to angular, more or less gelatinous, dissepiments fertile. Hyphal system monomitic, generative hyphae with clamps or simple-septa. 
Cystidia absent, cystidiols may be present. Basidia clavate, with 4-sterigmate. Basidiospores allantoid to cylindrical, smooth, thin-walled, hyaline, IKI-.

Remarks - Gloeoporus is undoubtedly closely related to Byssomerulius and separated mainly by the poroid hymenophore (merulioid in the latter). See also Ryvarden \& Melo (2017) and Bernicchia \& Gorjón (2020).

Gloeosoma Bres., Ann. Mycol. 18: 51, 1920.

Type species - Exidia vitellina Lév., Annls Sci. Nat., Bot., sér. 3 2: 219, 1844.

Basidiome cupulate, attached by a contracted base, texture gelatinous when fresh, drying cartilaginous, hymenophore smooth, trama distinctly duplex with a lower dense layer and a subiculum of looser consistency. Hyphal system monomitic, hyphae with clamps. Acanthophyses and hyphidia generally unbranched or with a few apical stout side branches. Basidia subclavate to clavate, with 4 large sterigmata. Basidiospores ovoid, echinulate, amyloid.

Remarks - Gloeosoma is easy to recognize by the large cupulate yellowish to orange and gelatinous basidiomes. Microscopically it has typical features of the genus Aleurodiscus s.l. Monotypic genus from Patagonia associated to Nothofagus species (Gorjón et al. 2013).

Gloeostereum S. Ito \& S. Imai, Trans. Sapporo nat. Hist. Soc. 13(1): 11, 1933.

Type species - Gloeostereum incarnatum S. Ito \& S. Imai, Trans. Sapporo nat. Hist. Soc. 13(1): 11, 1933.

Remarks - Monotypic genus. A synonym of Merulius Fr. according to Spirin \& Zmitrovich (2004).

Gloeotulasnella Höhn. \& Litsch., Wiesner Festschrift (Wien): 57, 1908.

Type species - Tulasnella cystidiophora Höhn. \& Litsch., Sber. Akad. Wiss. Wien, Math.naturw. Kl., Abt. 1 115: 1557, 1906.

Remarks - A synonym of Tulasnella J. Schröt.

Gloiodon P. Karst., Meddn Soc. Fauna Flora fenn. 5: 42, 1879.

Type species - Hydnum strigosum Sw., K. Vetensk-Acad. Nya Handl. 31(3): 250, 1810.

Basidiome annual, pileate to effuse-reflexed, upper surface strigose to smooth, dark brown to almost black; hymenophore formed by conical aculei. Hyphal system monomitic, hyphae with clamps, pigmented and some with occasional clamps, simulating skeletal hyphae. Gloeocystidia present. Basidia clavate, with 4-sterigmata, and a basal clamp. Basidiospores subglobose, hyaline, finely ornamented and strongly amyloid.

Remarks - Gloiodon is characterized by the brownish to greyish effuse-reflexed to pileate basidiome, and microscopically by the presence of gloeocystidia and amyloid ornamented basidiospores. Gloiodon is phylogenetically closely related to the stipitate Auriscalpium and Dentipratulum, and it is included in the Auriscalpiaceae among the russuloid lineage (Larsson 2007b).

Gloiothele Bres., Annls mycol. 18: 44, 1920.

Type species - Poria lamellosa Henn., Bot. Jb. 33: 35, 1904.

Basidiome resupinate, adnate, effused, hymenophore smooth to tuberculate. Hyphal system monomitic, hyphae with simple-septa. Cystidia (gloeocystidia) vesicular, with guttulate content, SA+. Hyphidia, smooth or encrusted, may be present. Basidia narrowly clavate to cylindrical, with 4-sterigmata. Basidiospores ellipsoid to subglobose, with a prominent apiculus, thin-walled, smooth, amyloid.

Remarks - Gloiothele includes monomitic species with simple-septate hyphae, gloeocystidia $\mathrm{SA}+$ and, smooth amyloid basidiospores (correspond to the Gloeocystidiellum lactescens group in Eriksson \& Ryvarden 1975). Wu (1996) included in Gloiothele also Vesiculomyces (with SAgloeocystidia) and it is obvious that both genera are closely related. 
Grammothele Berk. \& M.A. Curtis, J. Linn. Soc., Bot. 10(no. 46): 327, 1868.

Type species - Grammothele lineata Berk. \& M.A. Curtis, J. Linn. Soc., Bot. 10(no. 46): 327, 1868.

Basidiome annual, resupinate, adnate, effused, hymenial surface irregularly irpicoid to poroid and then partly labyrinthine to sinuous, pore surface, cream, bluish-grey to almost black with age or time pale brownish pinkish to pale umber brown, hymenium usually restricted to the base of the tubes. Hyphal system dimitic, generative hyphae with clamps, skeletal hyphae thick-walled to solid, dextrinoid at least in the outer parts, hyaline or darker with age and in some species coloured from the very beginning. Dendrohyphidia absent or present, both in the hymenium and in the dissepiments and the sterile tube walls. Basidia clavate, with 4-sterigmate, and a basal clamp. Basidiospores ellipsoid to cylindrical, thin-walled, smooth and non-amyloid, non-dextrinoid.

Remarks - The genus is related to Theleporus which is separated by being di-trimitic with branched, non-dextrinoid, arboriform skeletal hyphae (Ryvarden 1991).

Grammothelopsis Jülich, Biblthca Mycol. 85: 397, 1981.

Type species - Grammothele macrospora Ryvarden, in Ryvarden \& I. Johans., Prelim. Polyp. Fl. E. Afr. (Oslo): 43, 1980.

Basidiome annual, resupinate, effused, adnate, membranaceous, context homogeneous, hyphal strands lacking, hymenial surface poroid with angular pores, ochraceous to brownish. Hyphal system dimitic, generative hyphae with clamps, hyaline, cylindrical, thin-walled, arboriform hyphae with a straight long unbranched base and a branched terminal part, thick-walled, yellowish, with a weak to strong dextrinoid reaction. Cystidia absent. Dendrohyphia present. Basidia clavate, with 4-sterigmate, and a basal clamp. Basidiospores ellipsoid, smooth, thickwalled, with a distinct germ pore, inamyloid, strongly dextrinoid.

Remarks - Grammothele macrospora is separated from Grammothele by the arboriform vegetative hyphae and the thick-walled, dextrinoid basidiospores (Jülich 1981). Theleporus differs in the non-dextrinoid arboriform hyphae and thin-walled basidiospores.

Grandinia Fr., Epicr. syst. mycol. (Upsaliae): 527, 1838.

Type species - Thelephora granulosa Pers., Syn. meth. Fung. 576, 1801.

Remarks - A synonym of Xylodon (Pers.) Fr.

Grandiniella P. Karst., Hedwigia 34: 8, 1895.

Type species - Grandiniella livescens P. Karst., Hedwigia 34: 8, 1895. (=Corticium sordidum P. Karst., Meddn Soc. Fauna Flora fenn. 9: 65, 1882).

Remarks - A synonym of Phanerochaete P. Karst.

Grandiniochaete Rick, Annls mycol. 38(1): 59, 1940.

Type species - Grandiniochaete latecontexta Rick [as 'late-contextum'], Annls mycol. 38(1): 59, 1940.

Remarks - A name of uncertain application (see Hjortstam 1987a).

Grandinioides Banker, Mem. Torrey bot. Club 12(2): 179, 1906.

Type species - Peziza flava Sw., Nov. Gen. Spec. Pl.: 150, 1788.

Remarks - A synonym of Mycobonia Pat.

Granulobasidium Jülich, Persoonia 10: 328, 1979.

Type species - Corticium vellereum Ellis \& Cragin, Bull. Washburn Coll. Lab. Nat. Hist. 1: 66, 1885.

Basidiome resupinate, effused, adnate, membranaceous, hymenophore smooth or slightly tuberculate. Hyphal system monomitic, hyphae with clamps, hyaline, often guttulate. Cystidia absent. Basidia hyaline, cylindrical to narrowly clavate, with granular or guttulate contents, with 4- 
sterigmata, with a basal clamp. Basidiospores hyaline, thick-walled, rugose, guttulate, not amyloid, cyanophilous, with chlamydospores ellipsoid, thick-walled, cyanophilous and dextrinoid.

Remarks - Corticium vellereum has been considered a deviating species when it was included in Hypochnicium (Parmasto 1968, Eriksson \& Ryvarden 1976). It is characterized by lacking cystidia, the long tubular basidia with remarkable short sterigmata and granular contents, and the particular, irregularly uneven basidiospores; also, the presence of chlamydospores is remarkable. Phylogenetically, Granulobasidium is referred to as the Cyphellaceae with Chondrostereum, Cunninghammyces, and Gloeostereum but the relationships with these genera are still unclear (Larsson 2007b).

Granulocystis Hjortstam, Mycotaxon 25(1): 277, 1986.

Type species - Phanerochaete flabelliradiata J. Erikss. \& Hjortstam, in Eriksson, Hjortstam \& Ryvarden, Cortic. N. Eur. (Oslo) 6: 1073, 1981.

Remarks - A later homonym of Granulocystis F. Hindak 1977 (Algae). Leifia Ginns was proposed to replace Granulocystis Hjortstam (Ginns 1998). It is considered a synonym of Odonticium Parmasto by Zmitrovich (2001).

Griseoporia Ginns, Mycotaxon 20(2): 559, 1984.

Type species - Hexagonia carbonaria Berk. \& M.A. Curtis, Grevillea 1: 68. 1872.

Basidiomes resupinate to effuse-reflexed, rarely narrowly pileate, pores grey, 1-3 per mm, context to $1 \mathrm{~mm}$ thick, distinctly brown. Hyphal system dimitic, generative hyphae narrow with clamp connections, skeletal hyphae narrow. Basidiospores allantoid, rarely cylindrical, rather large, the spore wall smooth, thin, hyaline, nonamyloid, acyanophilous.

Remarks - Griseoporia was proposed for Hexagonia carbonaria Berk. \& M.A. Curtis that has traditionally been classified in Gloeophyllum P. Karst (Ginns 1984). Griseoporia carbonaria (Berk. \& M.A. Curtis) Ginns differs from other species in Gloeophyllum by the typically poroid hymenophore (lamellate to daedaleoid in Gloeophyllum s.str.) and from Osmoporus Singer in the resupinate to effuse-reflexed basidiomes, that are distinctly pileate in the latter. The separation of this genera from Gloeophyllum and Hispidaedalea Y.C. Dai \& S.H. He is phylogenetically supported (He et al. 2014).

Gyrodontium Pat., Essai Tax. Hyménomyc. (Lons-le-Saunier): 117, 1900.

Type species - Hydnum henningsii Bres., Bull. Soc. mycol. Fr. 6: XLVIII, 1890.

Basidiome resupinate to subpileate, hymenophore tuberculate to hydnoid. Hyphal system monomitic, hyphae with simple-septa, thin- to thick-walled, weakly cyanophilous. Cystidia absent. Basidia subclavate, thin-walled, with 4-sterigmata, and simple-septate at the base. Basidiospores ellipsoid, smooth, thick-walled, dextrinoid, cyanophilous.

Remarks - Gyrodontium is characterized by the tuberculate to hydnoid resupinate basidiome and thick-walled and dextrinoid basidiospores. Gyrodontium is morphologically and phylogenetically related to Coniophora in the Coniophoraceae (Larsson 2007b).

Gyrophanopsis Jülich, Persoonia 10: 329, 1979.

Synonym - Hyphodermopsis Jülich.

Type species - Pellicularia zealandica G. Cunn., Trans. Roy. Soc. New Zealand 81: 322, 1953.

Basidiome resupinate, effused, adnate, membranaceous, hymenophore smooth, margin thinning out, rhizomorphs absent. Hyphal system monomitic, hyphae with clamps, hyaline, distinct, cylindrical, thin- to somewhat thick-walled. Cystidia (septocystidia), hyaline, cylindrical, somewhat thick-walled, encrusted. Basidia hyaline, suburniform, with 4-sterigmata, and with a basal clamp. Basidiospores hyaline, ellipsoid, smooth, slightly thick-walled, inamyloid, cyanophilous.

Remarks - Gyrophanopsis is separated from Hypochnicium s.l. by the characteristically hyphoid encrusted septocystidia (Jülich 1979). Phylogenetic relationships are unclear. 
Gyrophora Pat., Hym. Eur. p. 143, 1887.

Type species - Boletus lacrymans Wulfen, in Jacquin, Miscell. Austriac. 2: 111, 1781.

Remarks - A later homonym of Gyrophora Ach. 1803 and a synonym of Serpula (Pers.) Gray.

Haematostereum Pouzar, Česká Mykol. 13(1): 13, 1959.

Type species - Thelephora sanguinolenta Alb. \& Schwein., Consp. fung. (Leipzig): 274, 1805.

Remarks - A synonym of Stereum Pers.

Hallenbergia Dhingra \& Priyanka, Mycotaxon 118: 289, 2011.

Type species - Hallenbergia singularis Dhingra \& Priyanka, Mycotaxon 118: 291, 2011.

Basidiome resupinate, adnate, effused, thin, ceraceous, hymenial surface smooth, farinose under the lens, continuous, some cracks developing on drying, margin not well differentiated. Hyphal system monomitic. generative hyphae thin-walled, clamped, basal hyphae irregularly branched and interwoven into a dense texture, subhymenial hyphae short-celled, compactly packed and appear like a cellular tissue. Cystidia absent. Basidia subclavate to suburniform, 4-sterigmate, basal clamp not observed. Basidiospores ellipsoid to ovoid or subglobose, smooth, with thickened walls, cyanophilous, inamyloid.

Remarks - According to Dhingra \& Priyanka (2011b) Hallenbergia resembles the genus Hypochnicium in having broadly ellipsoid to subglobose basidiospores with somewhat thick and cyanophilous walls. There are also affinities with Intextomyces in having a densely interwoven texture. The new genus differs from both genera by the peculiar hyphal texture, with a densely and irregularly branched lower part and a pseudoparenchymatous upper part. Moreover, it differs from Intextomyces in the way basidia are formed. In Intextomyces contiguus (P. Karst.) J. Erikss. \& Ryvarden, basidia are formed at the apex of penetrating, sinuous hyphae, while in Hallenbergia singularis the basidia are directly produced from the surface of the pseudoparenchymatic tissue.

Haloaleurodiscus N. Maek., Suhara \& K. Kinjo, Mycological Research 109 (7): 826, 2005.

Type species - Haloaleurodiscus mangrovei N. Maek., Suhara \& K. Kinjo, Mycological Research 109 (7): 827, 2005.

Basidiome annual, resupinate, effused. Hyphal system monomitic, hyphae with clamps. Gloeocystidia tubular, with a basal clamp, SA+. Dendrohyphidia present (in the original description with no indication to reactions in Melzer's reagent). Basidia clavate, sometimes sinuous, with 4sterigmata and a basal clamp. Basidiospores ellipsoid to cylindrical, warted, thin to slightly thickwalled, amyloid. Causing a white pocket rot.

Remarks - It is characterized by the ornamented and amyloid basidiospores, the presence of dendrohyphidia, and the white pocket rot. So far, monotypic genus with an only species growing on mangroves (Sonneratia alba Sm.). Phylogenetically it seems related to Scytinostroma and Dichostereum, more than other members of Aleurodiscus s.l. (as indicated by the generic name), and further investigation in this line can provide more date of the natural relationships (Maekawa et al 2005).

Haploporus Singer, Mycologia 35: 66, 1944.

Type species - Polyporus odorus Sommerf., Suppl. Fl. lapp. (Oslo): 275, 1826.

Synonym - Pachykytospora Kotl. \& Pouzar.

Basidiomes perennial, pileate, whitish and with a strong scent of anise, hymenophore tubular. Hyphal system trimitic, generative hyphae with clamps. Cystidia absent. Basidia clavate, tetrasterigmate, with a basal clamp. Basidiospores globose to ellipsoid, hyaline, asperulate or with longitudinal ridges, variably dextrinoid in Melzer's reagent. Causing a white heart rot of laying trees, preferably on Salix. Circumpolar in the boreal zone. 
Remarks - The genus is well characterized by its perennial basidiomes with trimitic hyphal system, and the dextrinoid basidiospores. According to Justo et al. (2017), Haploporus is classified in the Polyporaceae among the Polyporales. Crassisporus is phylogenetically closely related but differentiated by the smooth basidiospores (Ji et al. 2019).

Haplotrichum Link, in Willdenow, Sp. pl., Ed. 4 6(1): 52, 1824.

Type species - Haplotrichum capitatum Link, in Willdenow, Sp. pl., Ed. 4 6(1): 52, 1824.

Remarks - Anamorphic stage of Botryobasidium Donk. See Holubová-Jechová (1976).

Hastodontia (Parmasto) Hjortstam \& Ryvarden, Syn. Fung. (Oslo) 26: 49, 2009.

Basionym - Hyphodontia sect. Corticiodontia subsect. Hastodontia Parmasto, Consp. System. Corticiac. (Tartu): 122, 1968.

Type species - Peniophora hastata Litsch., Öst. bot. Z. 77(2): 130, 1928.

Basidiome resupinate, effused, adnate, hymenophore smooth to tuberculate. Hyphal system monomitic, all hyphae with clamps, distinct to thick-walled. Cystidia of two kind: 1) subulate cystidia; 2) enclosed moniliform cystidia. Basidia subcylindrical, with a median constriction, with 4-sterigmata and a basal clamp. Basidiospores cylindrical to subcylindrical, smooth, thin-walled, IKI-, CB-.

Remarks - Hastodontia is recognized among Hyphodontia s.l. by the combination of subulate and moniliform enclosed cystidia (Hjortstam \& Ryvarden 2009b). Phylogenetic relationships are unclear.

Heptasporium Bref., Unters. Gesamtgeb. Mycol. 14: 167, 1908.

Type species - Heptasporium gracile Bref., Unters. Gesammtgeb. Mykol. (Liepzig) 14: 167, 1908. (=Sistotrema brinkmannii? (Bres.) J. Erikss., K. Fysiogr. Sällsk. Lund. Förhandl. 18(8): 134, 1948).

Remarks - A synonym of Sistotrema Fr.

Hemmesomyces Gilb. \& Nakasone, Mycologia 95(3): 467, 2003.

Type species - Hemmesomyces puauluensis Gilb. \& Nakasone, Mycologia 95(3): 467, 2003.

Basidiome resupinate, hymenial surface smooth to tuberculate. Hyphal system monomitic, hyphae with clamps. Cystidia of two types: 1) fusiform, terminal or lateral, with short, knobby branches at base and appearing rooted, tapering to an acute point, arising from subiculum and subhymenium, walls at first hyaline and slightly thickened, then becoming brown, thick, lightly encrusted, sometimes dextrinoid in Melzer's reagent; 2) globose, ellipsoid, or broadly cylindrical, apex obtuse, attenuate or mammiform, arising from subiculum and subhymenium, often containing conspicuous refractive globules or oil-like materials, negative in sulfovanillin, walls hyaline, thin or slightly thick, smooth. Basidia clavate, 4-sterigmate, with a basal clamp. Basidiospores globose to subglobose, walls hyaline, smooth, slightly thick, negative in Melzer's reagent. Causing a white rot.

Remarks - Hemmesomyces is characterized by brown, rooted, fusiform cystidia, gloeocystidia (negative in sulfovanillin), globose to subglobose slightly thickened basidiospores, and clamped generative hyphae (Gilbertson \& Nakasone 2003). Monotypic genus. Not validly published, type not mentioned (?).

Hermanssonia Zmitr., Folia Cryptogamica Petropolitana 6: 100, 2018.

Type species - Phlebia centrifuga P. Karst., Meddn Soc. Fauna Flora fenn. 6: 10, 1881.

Remarks - A synonym of Phlebia Fr. Hermanssonia is here considered a synonym of Phlebia and waiting for phylogenetic and sexual studies I prefer to adopt a wide concept of Phlebia.

Hericium Pers., Neues Mag. Bot. 1: 109, 1794.

Synonym - Creolophus P. Karst.

Type species - Hydnum coralloides Scop., Fl. carniol., Edn 2 (Wien) 2: 472, 1772. 
Basidiome pileate, usually large, dimidiate to globose, with imbricate or gregarious growth, upper side tomentose to hirsute, hymenophore hydnoid. Hyphal system monomitic, hyphae with clamps. Gloeocystidia present. Basidia clavate, with 4-sterigmata, and a basal clamp. Basidiospores ellipsoid, slightly rugose or asperulate, amyloid.

Remarks - Hericium is characterized and easy to identify by the large pileate and fleshy hydnoid basidiome, microscopically by the presence of gloeocysitida and amyloid ornamented basidiospores. It is morphologically close related to Climacodon, but the latter differs in having inamyloid basidiospores. Phylogenetically, Hericium is classified in the Hericiaceae within the russuloid lineage, and very closely related to Laxitextum, with steroid basidiome (Larsson 2007b). Hydnum cirrhatum Pers., has been referred to Creolophus on the basis of the basidiospores that seem smooth at the light microscope, but they appear slightly rugose at SEM; it clusters also with other Hericium species; thus the generic separation is not supported (Larsson \& Larsson 2003).

Heteroacanthella Oberw., in Oberwinkler, Langer, Burdsall \& Tschen, Trans. Mycol. Soc. Japan 31(2): 208, 1990.

Type species - Heteroacanthella variabilis Oberw. \& Langer, in Oberwinkler, Langer, Burdsall \& Tschen, Trans. Mycol. Soc. Japan 31(2): 211, 1990.

Basidiome effused, dense, ceraceous, grey, with a distinct margin. Hyphal system monomitic, with clamp connexions in the type species (simple-septate in $H$. variabilis). Hymenium thin, densely agglutinated, composed of one or more layers of basidia and hyphidia arising from laterally branching subicular hyphae. Hyphidia variously cylindrical, clavate, or irregularly swollen, acanthoid, with numerous simple or branched apical processes. Basidia subglobose to pyriform or ellipsoid, acanthoid, with numerous simple processes up to $2 \mu \mathrm{m}$ long scattered around the apex, with one long sterigmata in the type species (one or usually two sterigmata in $H$. variabilis), occasionally producing a single, short, subsidiary sterigma and thus appearing furcate. Basidiospores globose to subglobose, smooth, hyaline, inamyloid, some producing secondary spores by replication.

Remarks - Heteroacanthella is characterized by the repetitive basidiospores, hyphidia and globose basidia with acanthoid protuberances, and with one or two long sterigmata (see Oberwinkler et al. 1990, Roberts 1998). It was referred to the new family Hetereacanthellaceae P. Roberts in the Exidiales (Roberts 1998) but it is included in this manual because of the aseptate basidia.

Heterobasidion Bref., Unters. Gesammtgeb. Mykol. (Liepzig) 8: 154, 1888.

Type species - Polyporus annosus Fr., Syst. mycol. (Lundae) 1: 373, 1821.

Basidiome perennial, resupinate to pileate, widely effused and tough; pileal surface at first light brown and finely tomentose, soon darker and smooth with a distinct thin black cuticle. Pore surface light cream, pores regular, round to angular, mostly small, context white to light cream, dark reddish brown with Melzer's reagent. Hyphal system dimitic, generative hyphae thin-walled, simple septate, skeletal hyphae dominating in the basidiome, dextrinoid, unbranched or with a few branches. Cystidia none. Basidia clavate, with 4-sterigmate, simple-septate at the base. Basidiospores broadly ellipsoid to globose, hyaline, thin to slightly thick-walled, finely asperulate, negative in Melzer's reagent. Causing a white rot of living conifers.

Remarks - Heterobasidion is characterized among the polypores by the dextrinoid skeletal hyphae, the simple septate generative hyphae, and the asperulate spores negative in Melzer's reagent. See also Ryvarden \& Melo (2017) and Bernicchia \& Gorjón (2020).

Heteromyces L.S. Olive, American J. Bot. 44: 432, 1957.

Type species - Sebacina fibrillosa Burt, Ann. Mo. bot. Gdn 13(3): 335, 1926.

Remarks - A later homonym of Heteromyces Müll. 1889 and a synonym of Oliveonia Donk.

Hirneola Fr. Syst. orb. veg. (Lundae) 1: 93, 1825, non Hirneola Fr. 1849. 
Type species - Peziza flava Sw., Nov. Gen. Spec. Pl.: 150, 1788.

Remarks - Donk (1941) proposed the conservation of Mycobonia Pat. 1894 against Hirneola Fr. 1825, both typified with Peziza flava to avoid confusion and save Hirneola Fr. 1849 (typified by Peziza nigra Fr.) for species belonging to Auriculariales.

Hispidaedalea Y.C. Dai \& S.H. He, in He, Vlasák \& Dai, Mycol. Progr. 13(3): 835, 2014.

Type species - not indicated.

Remarks - Nom. inval., Art. 40.1 (Shenzhen code). See He et al. (2014) for generic delimitation.

Hjortstamia Boidin \& Gilles, Bull. Soc. mycol. Fr. 118(2): 99, 2002.

Type species - Thelephora friesii Lév., Syst. Verz.: 17, 1854.

Basidiome resupinate, effuse-reflexed to pileate, upper sterile surface tomentose, brown to greyish coloured, hymenophore smooth to tuberculate, usually with brown colours and often with a pinkish tint. Hyphal system dimitic, generative hyphae with simple septa (some species have few clamps at the basal hyphal but never at the basidial base), skeletal (skeletoid) hyphae thick-walled and brown coloured. Cystidia (skeletocystidia or hymenial cystidial) pale brown, thick-walled, smooth or encrusted. Basidia clavate, with 4-sterigmata, without a basal clamp. Basidiospores cylindrical to ellipsoid, smooth, hyaline, IKI-, CB-.

Remarks - Hjortstamia was introduced for stereoid species with a dimitic hyphal system, generative hyphae without clamps, and astatocoenocytic nuclear behaviour (normal in Lopharia) (Boidin \& Gilles 2002). Hjortstamia is distinguished from Phanerochaete morphologically by brownish basidiome, subiculum with a loose texture with skeletal or skeletoid hyphae, and pale yellow to brownish skeletocystidia ( $\mathrm{Wu}$ et al. 2010). The distinction of a strictly monomitic or dimitic hyphal system in some Hjortstamia species is often difficult and intermediate stages are frequent. In some species, there are skeletoid brown hyphae with some sparse simple or secondary septa, that cannot be called true skeletals, but they serve, as the skeletals, to provide support in reflexed basidiome. However, it seems that Phanerochaete is not the natural place for this group of species, and according to molecular and morphological data we keep Hjortstamia as a different genus close related but distinct from Phlebiopsis, Rhizochaete and Phanerochaete in accordance with Wu et al. l.c.

Hornodermoporus Teixeira, Boletim do Instituto de Botânica, São Paulo 8: 21, 1993.

Type species - Polyporus martius Berk., Hooker's J. Bot. Kew Gard. Misc. 8: 198, 1856.

Basidiome annual to perennial, pileate to effused-reflexed, woody hard, pilei applanate to ungulate, glabrous, usually irregularly concentrically sulcate, dirty brown to black with a distinct crust. Pore surface white to dirty ochraceous; pores round; dissepiments thick, entire. Context pale buff to wood color, woody hard. Tubes concolorous with pore surface, hard corky. Hyphal system dimitic; generative hyphae bearing clamp connections; skeletal hyphae strongly dextrinoid, cyanophilous; tissues unchanged in $\mathrm{KOH}$. Cystidia present. Basidiospores ellipsoid, truncate, hyaline, thick-walled, smooth, strongly dextrinoid, $\mathrm{CB}+$.

Remarks - Hornodermoporus is similar to Perenniporia but differs by having a black crust at pileal surface, the presence of cystidia and oblong-ellipsoid basidiospores. Previously, species in the genus were treated in Perenniporia. Recent phylogenetic studies supported that Hornodermoporus is distinct from the Perenniporia s.str. clade (Zhao et al. 2013a).

Hydnochaete Bres., Hedwigia 35: 287, 1896.

Type species - Hydnochaete badia Bres., Hedwigia 35(5): 287, 1896. (=Hydnum peroxydatum Berk. ex Cooke, Grevillea 20 (no. 93): 1, 1891.

Remarks - Phylogenetically it may be considered a synonym of Hymenochaete Lév. (Baltazar et al. 2014). From a morphological system it is a useful entity to comprise those species of Hymenochaete with a hydnoid hymenophore. 
Hydnochaete Peck, Rep. N.Y. State Mus. 50: 113, 1897.

Type species - Hydnochaete setigera Peck, Ann. Rep. N.Y. St. Mus. 50: 113, 1898. (=Asterodon ferruginosus Pat., Bull. Soc. mycol. Fr. 10(3): 130, 1894).

Remarks - A homonym of Hydnochaete Bres. 1896 and a synonym of Asterodon Pat.

Hydnochaetella Sacc., Tab. Com. Gen. Fung. p. 11, 1898.

Type species - Hydnochaete setigera Peck, Ann. Rep. N.Y. St. Mus. 50: 113, 1898.

(=Asterodon ferruginosus Pat., Bull. Soc. mycol. Fr. 10(3): 130, 1894).

Remarks - A substitute for Hydnochaete Peck and a synonym of Asterodon Pat.

Hydnocristella R.H. Petersen, Česká Mykol. 25(3): 130, 1971.

Type species - Hydnum himantia Schwein., Schr. naturf. Ges. Leipzig 1: 104, 1822.

Basidiome resupinate, effused, hymenophore hydnoid, margin fibrillose with rhizomorphs. Hyphal system monomitic, hyphae with clamps, thin- to thick-walled, smooth or encrusted with cyanophilous warts. Cystidia absent. Basidia clavate, with 4-sterigmata, and a basal clamp. Basidiospores fusiform to subcylindrical, thin-walled, smooth, IKI-.

Remarks - Hydnocristella is characterized by the resupinate hydnoid basidiome and microscopically by the hyphae encrusted with cyanophilous warts, and by the inamyloid and smooth basidiospores (Petersen 1971). Molecular data support that Kavinia is restricted to species with ornamented basidiospores, whereas smooth-spored species are referred to Hydnocristella (Hosaka et al. 2006, Larsson 2007b). Kavinia, Hydnocristella and Ramaricium, are probably derived from Clavariadelphus, a clavarioid genus (Giachini et al. 2010).

Hydnodon Banker, Mycologia 5(6): 297, 1913.

Type species - Hydnum thelephorum Lév., Annls Sci. Nat., Bot., sér. 3 2: 204, 1844.

Remarks - A synonym of Trechispora P. Karst. Hydnodon was erected to accommodate a widespread, semistipitate hydnoid species with echinulate inamyloid basidiospores and ampullate swellings at the hyphal septa. It is restricted to tropical parts of America. For further comments see Ryvarden (2002).

Hydnofomes Henn., Engl. Bot. Jahrb. 28: 267, 1900.

Type species - Hydnofomes tsugicola Henn. \& Shirai, in Hennings, Bot. Jb. 28: 268, 1900. (=Fomes tinctorius Ellis \& Everh., Bull. Torrey bot. Club 22(7): 362, 1895).

Remarks - A synonym of Echinodontium Ellis. \& Everh.

Hydnomerulius Jarosch \& Besl, Pl. Biol. 3(3): 447, 2001.

Type species - Hydnum pinastri Fr., Novit. fl. svec. 2: 38, 1814.

Basidiome resupinate, effused, hymenophore smooth at first, but then typically hydnoid to irpicoid, yellowish to brownish-yellow, margin rhizomorphic with sclerotia. Hyphal system monomitic, hyphae with clamps. Cystidia absent. Basidia clavate, with 4-sterigmata, basally clamped. Basidiospores ellipsoid, smooth, thick-walled, usually yellow to brown coloured, weakly dextrinoid, cyanophilous.

Remarks - Jarosch \& Besl (2001) proposed the inclusion of Hydnum pinastri, traditionally classified in Leucogyrophana, in the new genus Hydnomerulius. It is closely related to the mushroom-forming fungi Paxillus and Gyrodon and to the gastroid Melanogaster in the Paxillineae (Binder \& Hibbett 2006). This separation is well supported by molecular data, but from a morphological point of view, Hydnomerulius differs from Leucogyrophana only in the clearly hydnoid hymenophore. However, we adopt here the separation of Hydnum pinastri from Leucogyrophana highlighting another an interesting example of a close relationship among resupinate, pileate-stipitate and gastroid forms in the Boletales. 
Hydnophanerochaete Sheng H. Wu \& C.C. Chen, Mycokeys 39: 85, 2018.

Type species - Phanerochaete odontoidea Sheng H. Wu, Bot. Bull. Acad. sin., Taipei 41(2): 169, 2000 (=Hydnophanerochaete odontoidea (Sheng $\mathrm{H}$. Wu) Sheng $\mathrm{H}$. Wu \& C.C. Chen, Mycokeys 39: 86, 2018).

Basidiome effused, adnate, ceraceous, hymenial surface at first buff, with age turning ochraceous to pale brown, slightly tuberculate to grandinioid when young, becoming odontioid to hydnoid with age, without color changes in $\mathrm{KOH}$. Aculei conical to cylindrical, ca. 1-4 per mm, up to $700 \mu \mathrm{m}$ long. Hyphal system essentially monomitic; generative hyphae simple-septate. Subiculum fairly uniform, composed of a basal layer, with compact texture; generative hyphae somewhat horizontal, colorless, thick-walled; quasi-binding hyphae present near substratum, colorless. Hymenial layer thickening. Trama of aculei of compact texture; generative hyphae somewhat vertical, colorless, thick-walled. Cystidia lacking but projecting hyphal ends in the hymenium may be present. Basidia clavate, 4-sterigmate. Basidiospores ellipsoid to cylindrical, smooth, thin-walled, inamyloid, non-dextrinoid, CB-.

Remarks - Hydnophanerochaete is morphologically similar to the genus Hydnophlebia (Telleria et al. 2017). Both genera have resupinate basidiomes with odontioid to hydnoid hymenial surface, a monomitic hyphal system, ordinarily simple-septate hyphae and similar basidiospore shape. However, Hydnophlebia has membranaceous basidiome usually with rhizomorphic margin, while Hydnophanerochaete has ceraceous basidiomes with fairly determinate margin, occasional single or multiple clamp connections are present in subicular or aculei hyphae of Hydnophlebia, whereas they are lacking in hyphae of Hydnophanerochaete, and, Hydnophlebia occasionally bears tubular to ventricose leptocystidia, which are lacking in Hydnophanerochaete. Little morphological differences exist between Hydnophanerochaete and Odontoefibula: both genera have a monomitic hyphal system with simple-septate hyphae and are lacking cystidia. However, Hydnophanerochaete is distinguished from Odontoefibula by its basidiomes without a color change in $\mathrm{KOH}$; additionally, its subiculum is compact, not dense.

Hydnophlebia Parmasto, Eesti NSV Tead. Akad. Toim., Biol. seer 16: 384, 1967.

Type species - Hydnum chrysorhizon Torr., Man. bot., Edn 3: 309, 1822.

Basidiome resupinate, membranous, reddish-orange, hymenophore hydnoid with aculei, margin with rhizomorphs. Hyphal system monomitic, hyphae with simple-septa. Cystidia cylindrical, tubular to ventricose, thin-walled, simple-septate at base. Basidia cylindrical to subclavate, with 4 sterigmata, basal clamp absent. Basidiospores ellipsoid, smooth, thin-walled, inamyloid, non-dextrinoid, CB-.

Remarks - Hydnophlebia was erected by Parmasto (1967) to accommodate Hydnum chrysorhizon Torr. A few years later, the type species was transferred to Phanerochaete P. Karst. (Budington \& Gilbertson 1973), and Hydnophlebia was neglected for a long time. According to Hjortstam \& Ryvarden (2009a), two species should be included in this genus of wood-inhabiting corticoid fungi: Hydnophlebia chrysorhizon (Torr.) Parmasto and Hydnophlebia omnivora (Shear) Hjortstam \& Ryvarden. Recently, Telleria et al. (2017) described three new species in the genus.

Hydnophysa Clem. Genera of Fung. p. 108, 1909.

Type species - Hydnofomes tsugicola Henn. \& Shirai, in Hennings, Bot. Jb. 28: 268, 1900. (=Fomes tinctorius Ellis \& Everh., Bull. Torrey bot. Club 22(7): 362, 1895).

Remarks - A superfluous substitute for Hydnofomes Henn. and a synonym of Echinodontium Ellis. \& Everh.

Hydnoporia Murrill, N. Amer. Fl. (New York) 9(1): 3, 1907.

Type species - Sistotrema fuscescens Schwein., Schr. naturf. Ges. Leipzig 1: 102, 1822 (=Sistotrema olivaceum Schwein, Schr. naturf. Ges. Leipzig 1: 101, 1822).

Remarks - Hydnoporia was proposed by Murrill for Sistotrema fuscescens, a synonym of Sistotrema olivaceum and actually considered as Hymenochaetopsis olivacea (Schwein.) S.H. He \& 
Jiao Yang. The name was not considered when Yang et al. (2016) proposed the name Hymenochaetopsis S.H. He \& Jiao Yang with Hymenochaetopsis tabacina (Sowerby) S.H. He \& Jiao Yang, as the generic type, to replace the invalid name Pseudochaete T. Wagner \& M. Fisch. Both, $H$. olivacea and $H$. tabacina cluster in the same clade delimited by Hymenochaetopsis in the vicinity to Hymenochaete as the closely related genus.

Hydnotrema Link, Handb. Erk. Gew. 3: 298, 1833.

Type species - Sistotrema confluens Pers., Neues Mag. Bot. 1: 108, 1794.

Remarks - A synonym of Sistotrema Fr.

Hydrabasidium Park.-Rhodes ex J. Erikss. \& Ryvarden, Cortic. N. Eur. 5: 896, 1978.

Type species - Hypochnus subviolaceus Peck, Ann. Rep. N.Y. St. Mus. 47: 25, 1894.

Remarks - A synonym of Scotomyces Jülich.

Hymenogramme Mont. \& Berk., Lond. J. Bot. 3: 329, 1844.

Type species - Hymenogramme javensis Mont. \& Berk., Lond. J. Bot. 3: 330, 1844.

Basidiome annual, resupinate, adnate, hymenial surface whitish to cream and consisting of long anastomosing ridges with the hymenium restricted to the furrows between the ridges. Hyphal system monomitic, generative hyphae with clamps. Cystidiols more or less, abundant in the hymenium. Basidia clavate and with 4-sterigmata. Basidospores broadly ellipsoid, thinwalled, smooth and non-amyloid.

Remarks - Hymenogramme has a unique surface with long sterile ridges and fertile furrows. This and the light fruitbodies with a monomitic hyphal system are the diagnostic characteristics (Ryvarden 1991).

Hymenochaete Lév., Annls Sci. Nat., Bot., sér. 3 5: 150, 1846.

Type species - Helvella rubiginosa Dicks., Fasc. pl. crypt. brit. (London) 1: 25, 1785.

Basidiome resupinate to pileate, hymenophore smooth to slightly tuberculate, more or less cracked, usually brown coloured, blackish in $\mathrm{KOH}$. Hyphal system monomitic, hyphae simpleseptate. Setae usually present. Basidia more or less tubular to clavate, with 4-sterigmata, simpleseptate at the base. Basidiospores smooth, thin-walled, hyaline, IKI-.

Remarks - Hymenochaete is placed in the Hymenochaetaceae with poroid genera such as Phellinus and Inonotus. It differs from other genera in the Hymenochaetaceae in having a smooth to tuberculate or hydnoid (Hydnochaete) hymenial surface, and from true stereoid fungi in the xanthocroic reaction of the basidiome and in the presence of setae (Baltazar et al. 2014).

Hymenochaetopsis S.H. He \& Jiao Yang, Mycol. Progr. 15(2/13): 2, 2016.

Type species - Auricularia tabacina Sowerby, Brit. Fung., pl. 25, 1797.

Basidiomes annual, resupinate, effused-reflexed or pileate, thin, membranous, coriaceous or corky. Hymenophore smooth, hydnoid, semi-lamellate or lamellate, more or less cracked with age, usually brown colored, turning black in $\mathrm{KOH}$. Hyphal system monomitic or subdimitic; generative hyphae simple-septate; setal hyphae present in some species. Setae usually abundant, reddishbrown, subulate, more or less encrusted. Basidia clavate to subcylindrical, with four sterigmata, simple-septate at the base. Basidiospores narrowly cylindrical to allantoid, hyaline, thin-walled, smooth, IKI-, CB-. Causing a white rot on angiosperm or gymnosperm wood.

Remarks - Hymenochaetopsis is very similar to Hymenochaete, it always formed a distinct sister clade with Hymenochaete in the phylogenetic trees of Hymenochaetaceae. Hymenochaetopsis have narrowly and shortly cylindrical to allantoid basidiospores with a maximum length of $7 \mu \mathrm{m}$ and width of $2.5 \mu \mathrm{m}$, which might be a useful character to distinguish it from Hymenochaete with usual cylindrical, ellipsoid to subglobose basidiospores (Yang et al. 2016). 
Hyphoderma Wallr., Fl. Crypt. Germ. (Nürnberg) 2: 576, 1833.

Type species - Thelephora setigera Fr., Elench. fung. (Greifswald) 1: 208, 1828.

Basidiome resupinate, effused, adnate, often ceraceous when fresh, hymenophore smooth, tuberculate to hydnoid, usually white coloured to yellowish with ochraceous or brown tints. Hyphal system monomitic, rarely dimitic, hyphae usually with clamps. Cystidia (leptocystidia) common. Basidia suburniform to subcylindrical, 4-sterigmata and a basal clamp. Basidiospores cylindrical, ellipsoid or subglobose, smooth, thin-walled, IKI-, with oily contents, abundant in herbarium dry specimens.

Remarks - Hyphoderma is a large and heterogeneous genus but usually easy to recognize by the large smooth basidiospores, basidia with a median constriction, and hyphae with usually conspicuous clamps. We follow Larsson (2007a) considering Peniophorella as a separated genus on the basis of molecular characters and in a microscopical level by the presence of more than one type of cystidia, echinulate cells (echinocysts or stephanocysts), clavate basidia and medium-large size basidiospores. According to the molecular study by Larsson I.c., Peniophorella is clearly separated from the rest of leptocystidiate Hyphoderma species, however, these phylogenetic analyses show that Hyphoderma is not monophyletic and further studies are necessary to explain adequately the generic diversity.

Hyphodermella J. Erikss. \& Ryvarden, Cortic. N. Eur. (Oslo) 4: 579, 1976.

Type species - Grandinia corrugata Fr., Hymenomyc. eur. (Upsaliae): 625, 1874.

Basidiome resupinate, effused, adnate, hymenophore grandinioid to odontioid, with small aculei and with a fibrillose apex, whitish to cream, margin determined. Hyphal system monomitic, hyphae with simple septa and thin walls. Cystidia absent, encrusted hyphal ends present. Basidia sinuous, clavate to suburniform, with 4-sterigmata and a simple basal septum. Basidiospores ellipsoid, smooth, thin-walled, IKI-.

Remarks - Hyphodermella is closely related to Hyphoderma but differing in the shape of basidia and lacking clamps (clamps are not also present in Hyphoderma capitatum and H. etruriae). The study of Larsson (2007b), based on molecular data, places Hyphodermella in the family Phanerochaetaeae. The genus Phanerochaete, generic type of this family, has generative simpleseptate hyphae even if some species have multiclamped septa at basal hyphae. Phanerochaetaeae would be closely related to a sister clade that includes, among others, simple septate species such as Byssomerulius, Ceriporia and Candelabrochaete.

Hyphodermopsis Jülich, Int. J. Mycol. Lichenol. 1: 28, 1982.

Type species - Kneiffia polonensis Bres., Ann. Mycol. 1: 103, 1903.

Remarks - A synonym of Gyrophanopsis Jülich.

Hyphodontia J. Erikss., Symb. Bot. Upsal. 16(1): 101, 1958.

Type species - Gonatobotrys pallidula Bres., Ann. Mycol. 1(2): 127, 1903.

Basidiome resupinate, effused, hymenophore smooth to odontioid or hydnoid. Hyphal system monomitic, all hyphae with clamps, subicular hyphae weakly dextrinoid and distinctly cyanophilous. Cystidia of two kinds: 1) Cystidia septate with more or less capitate apex and some clamped septa, 2) typical lagenocystidia (but few or absent in some species). Basidia subcylindrical, with median constriction, with 4-sterigmata and a basal clamp. Basidiospores subglobose to ellipsoid, smooth, thin-walled, hyaline, IKI-, CB-.

Remarks - Hyphodontia is considered here in a restricted sense including only those species with septocystidia and lagenocystidia (Hjortstam \& Ryvarden 2009b). In the traditional sense, Hyphodontia included a large number of species with many sterile elements and with an easily recognized more or less thick-walled hyphae with well delimited small circular clamps. Cladistic analyses by Langer (1994a) based on morphological and molecular characters suggested that Hyphodontia is not monophyletic and that cystidial morphology could be an indication of the species relationships; in addition, several molecular studies show that Hyphodontia is polyphyletic 
(Langer 2002, Binder et al. 2005, Larsson et al. 2006). Although we are reticent to split genera into small entities when there is limited morphological or molecular support, in the case of Hyphodontia, there are many evidences to consider it in a more restricted sense. However, the limits and relationships between most of the species in Hyphodontia s.l. are still unclear. There are many deviating species of Hyphodontia s.l. from the southern hemisphere with distinct cyanophilous and thick-walled spores, with transitional typical lagenocystidia to heavily encrusted cystidia, with tramal and large capitate cystidia (see Greslebin \& Rajchenberg 2000, Gorjón 2012) that does not fit satisfactory in the proposed genera of Hyphodontia s.str. A more detailed study is necessary, including species from both hemispheres, and combining morphological and molecular analysis, to have a more comprehensible vision of the Hyphodontia s.l. For recent studies see also Riebesehl \& Langer (2017), Yurchenko et al. (2020), Yurchenko \& Wu (2016).

Hyphodontiastra Hjortstam, Kew Bull. 54(3): 755, 1999.

Type species - Hyphodontiastra virgicola Hjortstam \& Melo, in Hjortstam, Kew Bull. 54(3): 756, 1999.

Basidiome resupinate, thin, loosely attached, hymenophore odontioid, aculei entire or fimbriate, fairly soft to fragile. Hyphal system monomitic, hyphae thin-walled, with clamps. Gloeocystidia tubular, thin-walled, more or less sinuous, with oily contents, yellowish in $\mathrm{KOH}$, brownish in sulphovanillin. Basidia tubular to narrowly clavate, basally slightly thick-walled, with four sterigmata and a basal clamp, basidia collapsing after spore discharge, the sterigmata turned inwards and difficult to discern. Basidiospores narrowly ellipsoid, smooth, thin-walled, IKI-, CB-.

Remarks - Hyphodontiastra is reminiscent of Hyphodontia but differing in the conspicuous gloeocystidia and tubular to clavate basidia. Gloeocystidia reminds those of Boidinia and Gloeocystidiellum s.l. Monotypic genus from Brazil (Hjortstam 1999).

Hyphodontiella Å. Strid, Kgl. norske vidensk. Selsk. Skr. 4: 19, 1975.

Type species - Hyphodontiella multiseptata Å. Strid, Kgl. norske vidensk. Selsk. Skr. 4: 19, 1975.

Basidiome resupinate, effused, hymenophore smooth, more or less porulose at first. Hyphal system monomitic, hyphae with both clamps and simple-septa, slightly thick-walled, not encrusted. Cystidia absent. Basidia clavate to subcylindrical, more or less constricted, with 4-sterigmata and a basal clamp. Basidiospores subfusiform to navicular, smooth, thin-walled, IKI-.

Remarks - Hyphodontiella resembles Hyphodontia in the nature of the hyphae, differing in the presence of secondary simple-septa and in the shape of the basidiospores (Eriksson \& Ryvarden 1976). According to molecular studies, Hyphodontiella clusters with clavarioid taxa, but there are no obvious morphological similarities to indicate that connection (Larsson 2007b).

Hyphoradulum Pouzar, Česká Mykol. 41: 26, 1987.

Type species - Hyphoradulum conspicuum Pouzar, Česká Mykol. 41(1): 26, 1987.

Remarks - A synonym of Pseudolagarobasidium J.C. Jang \& T. Chen. See Nakasone (2015).

Hypochnella J. Schröt., Krypt.-Fl. Schlesien 3(1): 402, 1888.

Type species - Hypochnella violacea Auersw. ex J. Schröt., Krypt.-Fl. Schlesien 3(1): 402, 1888.

Basidiome resupinate, adnate, hymenophore smooth, violaceous turning brownish when dry. Hyphal system monomitic, hyphae with simple-septa, branched at right angles, basal hyphae thickwalled, with brownish walls, subhymenial hyphae thin-walled, hyaline. Cystidia absent. Basidia cylindrical, with a slight median constriction, with 4-sterigmata and a simple basal septum. Basidiospores narrowly ellipsoid to ovoid, smooth, thick-walled, brownish to violet, with a slight amyloid reaction. 
Remarks - Hypochnella is characterized by the violaceous smooth basidiome, simple-septate and wide hyphae branched at right angles, and brownish to violaceous basal hyphae and basidiospores. The phylogenetic relationships of Hypochnella are unclear (Larsson 2007b).

Hypochniciellum Hjortstam \& Ryvarden, Mycotaxon 12: 176, 1980.

Type species - Leptosporomyces ovoideus Jülich, Willdenowia, Beih. 7: 203, 1972.

Basidiome resupinate, effused, pellicular (athelioid) or thin and membranaceous, hymenophore smooth, more or less byssoid. Hyphal system monomitic. Basal hyphae with thin to thickened walls, distinct, partly straight and uniform, hyaline, subhymenial hyphae thin-walled, faintly sinuous, all hyphae with clamps. Cystidia absent in the type species. Basidia fairly small, more or less clavate, moderately constricted, with 4-sterigmata. Basidiospores thick-walled, smooth, non-amyloid or dextrinoid, distinctly cyanophilous,

Remarks - Hypochniciellum was described to include Leptosporomyces ovoideus, an acystidiate species with thick-walled and cyanophilous basidiospores, and with no reaction in Melzer's reagent. Later, Hjortstam (1981) emended Hypochniciellum to include also species with greyish spore-wall in Melzer's reagent. The species having a distinct reaction in Melzer that Hjortstam added to Hypochiciellum, are now placed in Amylocorticiellum (Gorjón et al. 2011).

Hypochnicium J. Erikss., Symb. bot. upsal. 16(1): 100, 1958.

Type species - Thelephora bombycina Sommerf., Suppl. Fl. lapp. (Oslo): 284, 1826.

Basidiome resupinate, adnate, effused, hymenophore smooth, tuberculate or irregularly odontioid, margin indeterminate. Hyphal system monomitic, hyphae with clamps, thin- to thickwalled. Cystidia present or absent. Basidia cylindrical to narrowly clavate, usually constricted, with a basal clamp, with 4-sterigmata. Basidiospores ellipsoid to globose, distinctly thick-walled, smooth or warted, IKI-, cyanophilous.

Remarks - Hypochnicium in an old sense included species with thick-walled, cyanophilous, smooth or warted basidiospores (Boidin \& Lanquetin 1971, Eriksson \& Ryvarden 1976). Some species have been separated from Hypochnicium: Kneiffia polonensis has been included in Gyrophanopsis due to the hyphoid septate and encrusted cystidia; Gloeocystidium analogum in Gloeohypochnicium on the basis of the SA+ gloeocystidia; Peniophora detritica in Lagarobasidium for its evident affinities to Hyphodontia (Larsson 2007b); and Corticium vellereum in Granulobasidium by the irregularly warted basidiospores and the presence of chlamydospores.

Hypochnopsis P. Karst., Bidr. Känn. Finl. Nat. Folk 48: 442, 1889.

Type species - Hypochnopsis mustialaensis (P. Karst.) P. Karst., Bidr. Känn. Finl. Nat. Folk 48: 442, 1889.

Remarks - A synonym of Amaurodon J. Schröt.

Hypochnus Fr., Observ. Mycol. (Havniae) 2: 278, 1818.

Type species - Corticium ferrugineum Pers., Observ. Mycol. (Havniae) 2: 18, 1800.

Remarks - A synonym of Tomentella Pers. ex Pat.

Incrustoporia Domański, Acta Soc. Bot. Pol. 32: 737, 1963.

Type species - Poria stellae Pilát, Sb. Nár. Mus. v Praze, Rada B, Prír. Vedy 9(2): 107, 1953.

Remarks - Incrustoporia has usually been regarded as a synonym of Skeletocutis Kotl. \& Pouzar, but, phylogeny of genera in the Incrustoporiaceae is actually not settled (Justo et al. 2017).

Inflatostereum D.A. Reid, Nova Hedwigia, Beih. 18: 143, 1965.

Type species - Thelephora glabra Lév., Annls Sci. Nat., Bot., sér. 3 5: 147, 1846.

Basidiome discrete, coriaceous, flabelliform, pileus tending to split radially into wedgeshaped segments, glabrous, hymenial surface smooth, stipe lateral, very short or rudimentary, attached to the substrate by a thin, closely appressed, creamy-ochre coloured mycelial disc. Hyphal 
system mono- to dimitic, generative hyphae with clamps, thin- to thick-walled, some segments become conspicuously inflated up to $25 \mu \mathrm{m}$ wide, skeletal hyphae in the stipe and basal portion of the basidiome. Cystidia and gloeocystidia absent. Basidia clavate, often with one large oil globule or a number of smaller guttules in the apical portion, 4-spored. Basidiospores ellipsoid, smooth, hyaline, nonamyloid.

Remarks - Inflatostereum is characterized by the inflated hyphae and dimitic hyphal system. It seems close to Stereopsis but the latter differs in the monomitic hyphal system and non-inflated hyphae (Ryvarden 2010).

Inonotus P. Karst., Meddn Soc. Fauna Flora fenn. 5: 39, 1879.

Type species - Boletus hispidus Bull., Herb. Fr. (Paris) 4: tab. 210, 1784.

Basidiome annual, resupinate, effused-reflexed, sessile, or stipitate, tissue yellowish-to reddish-brown, darkening in $\mathrm{KOH}$; pileal surface hirsute, hispid, tomentose, or glabrous, yellowish to reddish-brown, often darkened and rimose in age; context brown, soft-fibrous to tough-corky. Hymenophore tubular with round to angular pores. Hyphal system monomitic to dimitic, hyphae simple-septate, in most species ranging from thin-walled and almost hyaline to thick-walled and brownish in $\mathrm{KOH}$, much branched, binding hyphae present in some species. Setal hyphae present in context or trama of some species; hymenial setae present in most species, usually hymenial or subhymenial in origin, subulate to ventricose, pointed, pale to dark brown in $\mathrm{KOH}$. Basidia clavate to broadly ellipsoid, 4-sterigmate, simple-septate at the base. Basidiospores narrowly ellipsoid to ovoid or subglobose, hyaline or golden to reddish brown, smooth, negative to variably dextrinoid in Melzer's reagent. Causing a white rot of living and dead conifers and hardwoods.

Remarks - Inonotus is a large genus of poroid Hymenochaetaceae, with soft basidiomes and a usually monomitic hyphal system (dimitic in Phellinus). We include it in this manual just to key out a large group of resupinate polypores. For more information, the reader is referred to specific polypore works (Bernicchia 2005, Bernicchia \& Gorjón 2020, Ryvarden 2005, Ryvarden \& Gilbertson 1993, Ryvarden \& Melo 2014, 2017).

Intextomyces J. Erikss. \& Ryvarden, Cortic. N. Eur. (Oslo) 4: 735, 1976.

Type species - Corticium calceum subsp. contiguum P. Karst., Acta Soc. Fauna Flora fenn. 2(1): 39, 1881.

Basidiome resupinate, effused, adnate, ceraceous, hymenophore at first smooth, then slightly tuberculate or odontioid, whitish or greyish, margin determinate. Hyphal system monomitic, hyphae clamped, thin-walled, irregularly ramified and densely interwoven. Cystidia absent or present (originally described without cystidia). Basidia suburniform, basally continuing in a narrow-stalked hypha, with a basal clamp. Basidiospores ellipsoid to subangulate, thick-walled, IKI-, cyanophilous.

Remarks - Intextomyces was segregated from Hypochnicium by Eriksson \& Ryvarden (1976) according to the different ceraceous structure of the basidiome with densely interwoven hyphae and with a different development. It is recognized, among other characters, by the stalked basidia and the subangulate thick-walled basidiospores, very abundant in herbarium dry specimens. The molecular study by Larsson (2007b) does not clarifies its phylogenetic relationships.

Irpex Fr., Elench. fung. (Greifswald) 1: 142, 1828.

Type species - Sistotrema lacteum Fr., Observ. mycol. (Havniae) 2: 266, 1818.

Basidiome annual, resupinate, effused or slightly effuse-reflexed, abhymenial surface whitish, tomentose, hymenophore poroid to hydnoid, with irregular to irpicoid pores. Hyphal system dimitic, generative hyphae simple-septate, skeletal hyphae thick-walled, aseptate. Cystidia (pseudocystidia or skeletocystidia) present, thick-walled, with encrusted terminal part. Basidia clavate, with 4-sterigmata and a simple basal septum. Basidiospores cylindrical, smooth, thinwalled, IKI-. 
Remarks - Irpex is usually well distinguished by the combination of micro- and macromorphological features. Irpex differs mainly from Steccherinum mainly in the irregularly irpicoid hymenophore whereas in the latter the hymenophore is formed by well-defined cylindrical aculei. In addition, most of the Steccherinum species have clamped generative hyphae. Phylogenetically Irpex and Steccherinum belong to the Polyporaceae but two different clades; Irpex is included in the Byssomerulius family with other clampless species, whereas Steccherinum belongs to the Meruliaceae with Junghuhnia as the closest related genus (Larsson 2007b). Irpex has traditionally been considered as a monotypic genus with the exclusion of many species (Maas Geesteranus 1974), but recently three more species have been added: Irpex cremicolor Miettinen, Niemelä \& Ryvarden from Scandinavia (Miettinen et al. 2007), Irpex hydnoides Y.W. Lim \& H.S. Jung (Lim \& Jung 2003), and Irpex hacksungii J.S. Lee \& Y.W. Lim (Lee et al. 2008) both from Korea. Kotiranta \& Saarenoksa (2002) transferred most of the Steccherinum species to Irpex, but at the time present we prefer to maintain the distinction, considering Irpex in the strict sense of a clampless genus with irregularly poroid to irpicoid hymenophore. Flavodon Ryvarden is another genus to consider, and often is synonymized to Irpex. Flavodon flavus, the generic type, is usually easily to distinguish by the yellowish to cinnamon hydoid to subporoid hymenophore that turns red in $\mathrm{KOH}$.

Irpicodon Pouzar, Folia geobot. phytotax. 1: 371, 1966.

Type species - Sistotrema pendulum Alb. \& Schwein., Consp. fung. (Leipzig): 261, 1805.

Basidiome pileate, dimidiate to flabelliform, often with imbricate growth, hymenophore hydnoid to sublamellate, margin lobed. Hyphal system monomitic, hyphae with clamps, thin- to slightly thick-walled in the trama. Cystidia absent. Basidia clavate, with 4-sterigmata, and a basal clamp, arranged in a dense palisade. Basidiospores suballantoid, smooth, thin-walled, amyloid.

Remarks - Irpicodon is characterized by the pileate hydnoid basidiome and amyloid basidiospores; Pouzar (1966b) pointed out a relationship with Amylocorticium and Anomoporia, both also with amyloid basidiospores. This supposition is confirmed by molecular data and Irpicodon is included in the Amylocorticiaceae with Amylocorticium, Anomoporia, Amylocorticiellum, Amyloxenasma and Plicaturopsis among others; with the last genus it shares also the pileate basidiome (Larsson 2007b).

Jaapia Bres., Ann. mycol. 9: 428, 1911.

Type species - Jaapia argillacea Bres., Annls mycol. 9(4): 428, 1911.

Basidiome resupinate, effused, adnate, hymenophore porulose to flocculose, pilose by protuding cystidia. Hyphal system monomitic, hyphae with clamps, thin-walled. Cystidia tubular, thick-walled, not encrusted. Basidia clavate, with 4-sterigmata, and a basal clamp. Basidiospores fusiform, smooth, thick-walled, hyaline to yellowish, dextrinoid, cyanophilous.

Remarks - Jaapia is characterized by the tubular thick-walled cystidia and the thick-walled dextrinoid and cyanophilous basidiospores. It includes only two well morphologically and phylogenetically delimited species, closely related to Coniophora and Serpula but in a distinct basal clade of the Boletales (Larsson 2007b). Jaapia forms the order Jaapiales as the sister group to the rest of the Agaricomycetidae, suggesting that the greatest radiation of pileate-stipitate mushrooms resulted from resupinate ancestors (Binder et al. 2010).

Jacksonomyces Jülich, Persoonia 10(3): 329, 1979.

Type species - Peniophora phlebioides H. S. Jacks. \& Dearden, Can. J. Res. (C) 27: 150, 1949.

Remarks - A synonym of Phlebia Fr. The type of Jacksonomyces has the typical ceraceous basidiome of Phlebia but deviates in its stalked-clavate basidia, partly pleurobasidioid, and rather small basidia. Peniophora phlebioides on the basis of morphological characters and interfertility tests, is considered to be a synonym of Phlebia subserialis. 
Junghuhnia Corda, Ann. Stud. Mycol. 195, 1842.

Type species - Laschia crustacea Jungh., Verh. Batav. Gerdotsch 17(2): 75, 1839.

Basidiome annual to perennial, resupinate to effuse-reflexed, hymenophore poroid, variable in colour, pores small, round to irregular. Hyphal system dimitic, generative hyphae with clamps, thin-walled, hyaline, skeletal hyphae dominant, hyaline, thick-walled to almost solid. Cystidia present as skeletocystidia, arising in the trama and projecting above the basidial layer, encrusted in the apical part. Basidia clavate, 4-stergimata, with a a basal clamp. Basidiospores cylindrical to ellipsoid, smooth, thin-walled, hyaline, IKI-. On dead wood or conifers and hardwoods, causing a white rot.

Remarks - It is very close related to Steccherinum, sharing the same microfeatures and only distinguished by the poroid hymenial surface. In recent studies Junghuhnia is treated as a synonym of Steccherinum (Miettinen \& Ryvarden 2016, Westphalen et al. 2018).

Kavinia Pilát, Stud. Bot. Čechoslav. 1: 3, 1938.

Type species - Caldesiella sajanensis Pilát, Bull. trimest. Soc. mycol. Fr. 52(3): 329, 1937 (= Hydnum alboviride Morgan, J. Cincinnati Soc. Nat. Hist. 10: 12, 1887).

Basidiome resupinate, effused, hymenophore hydnoid, margin fibrillose with rhizomorphs. Hyphal system monomitic, hyphae with clamps, thin- to thick-walled, usually smooth. Cystidia absent. Basidia clavate, with 4-sterigmata, and a basal clamp. Basidiospores fusiform to subcylindrical, with slightly thickened walls, and with cyanophilous warts.

Remarks - Kavinia is characterized by the hydnoid resupinate basidiome and microscopically by the fusiform basidiospores ornamented with cyanophilous warts. It is closely related to Hydnocristella but phylogenetically and morphologically well separated by the smooth basidiospores in the latter (Larsson 2007b).

Kneiffia Fr., Fl. Scan. p. 340: 1835.

Type species - Thelephora setigera Fr., Elench. fung. (Greifswald) 1: 208, 1828.

Remarks - A homonym of Kneiffia Spach. (Onagraceae) and a synonym of Hyphoderma Wallr.

Kneiffiella Underw., Bull. Torrey bot. Club 24: 205, 1897.

Type species - Thelephora setigera Fr., Elench. fung. (Greifswald) 1: 208, 1828.

Remarks - A substitute for Kneiffia Fr. and a homonym of Kneiffiella P. Karst.

Kneiffiella P. Karst., Bidr. Känn. Finl. Nat. Folk 48: 371, 1889.

Type species - Hydnum barba-jovis Bull. [as 'barba-jobi'], Herb. Fr. 11: tab. 481, fig. 2, 1791.

Basidiome resupinate, effused, hymenophore smooth to usually odontioid or hydnoid. Hyphal system monomitic (pseudodimitic), hyphae with or without clamps, thin- to thick-walled. Cystidia (pseudocystidia) numerous, tubular, thick-walled, arising from the subiculum. Basidia clavate to cylindrical with median constriction, with 4 sterigmata, with or without a basal clamp. Basidiospores subglobose to ellipsoid, smooth, thin-walled, hyaline, IKI-, CB-.

Remarks - Kneiffiella is morphologically and phylogenetically close to other Hyphodontia s.l. species but it is a valid and useful genus for those Hyphodontia species with thick-walled tubular cystidia of tramal origin. Larsson et al. (2006) recovered Kneiffiella in the Hymenochaetales. For a modern taxonomic concept see Riebesehl \& Langer (2017) and Yurchenko \& Wu (2016).

Koleroga Donk, Fungus, Wageningen 28: 35, 1958.

Type species - Koleroga noxia Donk, Fungus, Wageningen 28: 35, 1958.

Remarks - A synonym of Ceratobasidium D.P. Rogers. 
Korupella Hjortstam \& P. Roberts, Kew Bull. 55: 817, 2000.

Type species - Korupella denticulata Hjortstam \& P. Roberts, Kew Bull. 55: 817, 2000.

Basidiome resupinate, effuse, pellicular, hymenophore hydnoid. Hyphal system monomitic, hyphae simple-septate, thin- to thick-walled in the subiculum, some dextrinoid. Cystidia absent. Basidia narrowly clavate, with 4-sterigmata, with a simple basal-septum. Basidiospores oblong, smooth, thin-walled, inamyloid, some weakly dextrinoid (pale orange) and cyanophilous.

Remarks - Korupella was described for an African species with hydnoid hymenophore, unclamped hyphae, lack of cystidia, small basidiospores some of them are weakly dextrinoid and cyanophilous (Roberts 2000). Except for the reaction of the basidiospores, it is similar to species in Phanerochaete.

Kurtia Karasiński, Index Fungorum 140: 1, 2014.

Type species - not indicated.

Remarks - Nom. inval., Art. 40.6 (Shenzhen code). Karasiński (2014) indicated after the generic description 'Corticium argillaceum; here designated' but with no specific generic type designation. The generic name Kurtia was intended to accommodate Corticium argillaceum Bres., Gloeocystidium macedonicum Litsch., and Hyphoderma magnargillaceum Boidin \& Gilles.

Lachnocladium Lév., Considér. Mycol.: 108, 1846.

Type species - Eriocladus brasiliensis Lév., Annls Sci. Nat. Bot. Sér. 3(5): 159, 1846.

Remarks - A clavarioid genus belonging to the Lachnocladiaceae related to Scytinostroma, Gloiothele, Vesiculomyces, and Asterostroma (see Larsson \& Larsson 2003, Leal-Dutra et al. 2018).

Laeticorticium Donk, Fungus, Wageningen 26: 16, 1956.

Type species - Corticium roseum Pers., Neues Mag. Bot. 1: 111, 1794.

Remarks - A superfluous synonym of Corticium Pers.

Laetisaria Burds., Trans. Br. mycol. Soc. 72(3): 420, 1979.

Type species - Hypochnus fuciformis McAlpine, Annls mycol. 4(6): 549, 1906; anamorph as Isaria fuciformis Berk., J. Linn. Soc., Bot. 13: 175, 1873.

Basidiome effused, pellicular, hymenophore smooth. Hyphal system monomitic, hyphae simple-septate, with broad hyphae, up to $10 \mu \mathrm{m}$ diam. Cystidia absent, hyphidia simple, not branched. Probasidia sphaeropedunculate, metabasidium cylindrical to clavate, with 4-sterigmate, with a simple basal septum. Basidiospores ovoid, hyaline, thin-walled, IKI-, CB-.

Remarks - Laetisaria is a genus to accommodate Hypochnus fuciformis, an effused corticioid species related to Dendrocorticium, but differing in the simple-septate hyphae and not ramified hyphidia (Burdsall 1979). Phylogenetically it is classified in the Corticiaceae s.str. confirming the relationships supposed by Burdsall. The anamorph of L fuciformis is a clavarioid filamentous structure growing on grasses, where conidia formation has been reported by Berkeley (1873), Smith (1884), Massee (1893), and Cunningham (1954) but not by Burdsall l.c. Some nomenclatural confusion exists in the epithet "fuciformis", resolved by Burdsall l.c as follow. Berkeley (1873) proposed the name Isaria fuciformis Berk. for the clavariod anamorph stage. McAlpine (1906) found the perfect stage and proposed a combination as Hypochnus fuciformis (Berk.) McAlpine, but this combination was invalid because the teleomorphic name was typified by an anamorph. However, because McAlpine provided a description of the teleomorph, he is crediting with describing a new species of Hypochnus, that should be cited as Hypochnus fuciformis McAlpine. In addition, Wakefield (1917), combined Isaria fuciformis in Corticium, as C. fuciformis (Berk.) Wakef., thus in the same McAlpine situation, the name was not validly published. Because Wakefield's species is not conspecific with $H$. fuciformis, it should be regarded as a new species validly published, and the name cited as Corticium fuciformis Wakef. Wakefield's species is an Athelia species, similar to Athelia singularis Parmasto (Parmasto 1967), and because, A. singularis 
was subsequently described, C. fuciformis has priority, and it was stated by Burdsall l.c. as Athelia fuciformis (Wakef.) Burds.

Lagarobasidium Jülich, Persoonia 8: 84, 1974.

Type species - Odontia pruinosa Bres., Ann. mycol. 18(1/3): 43, 1920.

Remarks - A synonym of Xylodon (Pers.) Gray. Viner et al. (2018) showed that Odontia pruinosa, the type of Lagarobasidium, belongs in Xylodon and is a sister species to $X$. detriticus. Molecular and morphological information show that the traditional concept of Lagarobasidium detriticum (Bourdot) Jülich covers at least two species, Xylodon detriticus (Bourdot) K.H. Larss., Viner \& Spirin from Europe and $X$. pruinosus (Bres.) Spirin \& Viner, with known distribution in Europe and North America (Viner et al. 2018).

Larssoniporia Y.C. Dai, Jia J. Chen \& B.K. Cui, Persoonia 37: 26, 2015.

Type species - Fomes tropicalis Cooke, Grevillea 15 (73): 22, 1886.

Basidiome annual to perennial, pileate, effused-reflexed or resupinate, woody hard when dry. Pileal surface ochraceous beige to dark brown, usually glabrous. Pore surface cream, grey, alutaceous to brown; pores large to small, irregular, round to angular. Context clay-buff, rusty to brown, hard corky. Tubes tough. Hyphal system dimitic, generative hyphae with clamp connections, skeletal hyphae dextrinoid. Gloeocystidia present. Cystidia with crystals at tips present. Basidiospores broadly ellipsoid to subglobose, hyaline, thin- to slightly thick-walled, finely asperulate, $\mathrm{IKI}+, \mathrm{CB}-$ or $\mathrm{CB}+$. Causing a white rot.

Remarks - Larssoniporia is characterized by its woody hard basidiomes when dry, tough tubes, dextrinoid skeletal hyphae, presence of cystidia with crystals at tips and gloeocystidia, finely asperulate and amyloid basidiospores, and by presenting a distribution in the tropics. In the phylogeny of Chen et al. (2016), Larssoniporia tropicalis (Cooke) Y.C. Dai, Jia J. Chen \& B.K. Cui, the generic type, was present as a single lineage distant from the Wrightoporiaceae clade which was also shown by Larsson \& Larsson (2003).

Laurilia Pouzar, Česká Mykol. 13: 14, 1959.

Type species - Stereum sulcatum Burt, Ann. Rep. Reg. St. N.Y. 54: 154, 1901.

Basidiome perennial, resupinate to effuse-reflexed, with a corky consistence, hymenophore smooth to tuberculate. Hyphal system trimitic, generative hyphae with clamps, hyaline, skeletal hyphae subhyaline to pale brown, binding hyphae more or less hyaline. Cystidia (metuloids) thickwalled, encrusted. Basidia clavate, with 4-sterigmata, and a basal clamp. Basidiospores globose to subglobose, minutely echinulate thin- to slightly thick-walled, hyaline, amyloid.

Remarks - Laurilia is characterized by the trimitic hyphal system (a very rare feature among the corticioid fungi), the presence of metuloids, and by the amyloid echinulate basidiospores. It is phylogenetically included in the Echinodontiaceae among the russuloid clade with other corticioid genera as Amylostereum (Larsson \& Larsson 2003, Larsson 2007b). Monotypic genus. For further comments and phylogenetic analyses see Liu et al. (2017).

Lauriliella S.H. He \& Nakasone, Mycologia 109 (4): 571, 2017.

Type species - Stereum taxodii Lentz \& H.H. McKay, Mycologia 52: 262, 1961.

Basidiome perennial, effuse-reflexed, pileate or umbonate, woody hard. Upper surface velutinous, grayish brown to dark brown, sulcate, becoming glabrous, black, rimosus with age; margin acute to blunt, thickening with age. Hymenophore smooth to tuberculate, grayish orange, brownish orange to light brown; margin sterile, distinct. Context brownish orange to light brown, woody hard. Hyphal system dimitic. Generative hyphae thin- to thick-walled, hyaline, nodoseseptate, with scattered secondary simple septa. Skeletal hyphae dominant, thick-walled to subsolid, light yellow, unbranched. Cystidia hyphoid to clavate, thick-walled, apically encrusted, embedded or slightly projected. Basidia clavate, hyaline, with 4 sterigmata and a basal clamp connection. Basidiospores broadly ellipsoid to subglobose, hyaline, thick-walled, echinulate, amyloid. 
Associated with white stringy rot to brown powdery rot in pockets, often associated with living trees of Cupressaceae.

Remarks - Lauriliella creates large pockets of decayed wood scattered in the heartwood of Taxodium and Chamaecyparis that is somewhat stringy or laminated, whereas Laurilia causes a white stringy rot or white pocket rot of dead coniferous wood. The hymenophore is light yellow or pink to salmon-colored in Laurilia but gray, orange, or brown in Lauriliella. Microscopically, unbranched skeletal hyphae are dominant in the context of Lauriliella whereas in Laurilia unbranched skeletals and richly branched binding hyphae are present (Liu et al. 2017).

Lawreymyces Lücking \& Moncada, Fungal Diversity 84: 133, 2017.

Type species - Lawreymyces palicei Lücking \& Moncada, Fungal Diversity 84: 135, 2017.

Remarks - Lawreymyces is a genus proposed on by a cryptic (no physical type specimens preserved except for the corresponding host lichens, which, however, do not show the features of the fungus), lichenicolous basidiomycete in the family Corticiaceae, on lichens of the family Verrucariaceae, known specifically from the genera Agonimia and Normandina; consistently differing from the currently resolved sister clade formed by the related genera Erythricium and Marchandiobasidium (Lücking \& Moncada 2017).

Lawrynomyces Karasiński, Acta Mycol. 48(1): 6, 2013.

Type species - Hyphoderma capitatum J. Erikss. \& Å. Strid, in Eriksson \& Ryvarden, Cort. N. Eur. 3: 461, 1975.

Basidiome resupinate, effused, adnate, thin. Hymenophore even, margin indeterminate, without rhizomorphs. Hyphal system monomitic. Hyphae with simple septa, thin to thick-walled, hyaline. Cystidia tubular, with capitate apex. Hyphidia sometimes present. Basidia suburniform to subcylindrical and constricted, more or less pedunculate, basally without clamps, with (2-)4 prominent sterigmata. Basidiospores relatively large, broadly ellipsoid to subglobose, with slightly thickened walls and distinct apiculus, smooth, IKI-, CB-.

Remarks - Lawrynomyces was proposed to accommodate $H$. capitatum, a deviating species in Hyphoderma by the simple-septate hyphae. It belongs to the Rickenella clade in the Hymenochaetales (Karasiński 2013).

Laxitextum Lentz, U.S. Dept. Agric. Monogr. 24: 18, 1956.

Type species - Thelephora bicolor Pers., Syn. meth. fung. (Göttingen) 2: 568, 1801.

Basidiome resupinate an effuse-reflexed (stereoid), upper sterile surface tomentose, hymenophore smooth, whitish. Hyphal system monomitic, hyphae with clamps, thin-walled, brown in the trama. Cystidia (gloeocystidia) cylindrical to subulate, frequently constricted, enclosed or slightly projecting. Basidia tubular to clavate, with 4-sterigmata, and with a basal clamp. Basidiospores ellipsoid to subglobose, finely asperulate to echinulate, amyloid.

Remarks - Laxitextum is characterized by the stereoid basidiome, the presence of gloeocystidia and by the minutely echinulate amyloid basidiospores. Molecular studies by Larsson \& Larsson (2003) and Larsson et al. (2004) show that Laxitextum is closely related to Hericium in the russuloid clade.

Lazulinospora Burds. \& M.J. Larsen, Mycologia 66(1): 97, 1974.

Type species - Lazulinospora wakefieldiae Burds. \& M.J. Larsen, Mycologia 66(1): 98, 1974. Remarks - A synonym of Amaurodon J. Schröt.

Leaia Banker, Mem. Torrey Bot. Club 12: 175, 1906.

Type species - Leaia piperata Banker, Mem. Torrey bot. Club 12(2): 175, 1906, (=Hydnum strigosum Sw., K. Vetensk-Acad. Nya Handl. 31: 250, 1810).

Remarks - A synonym of Gloiodon P. Karst. 
Leifia Ginns, Mycologia 90(1): 19 (1998)

Type species - Phanerochaete flabelliradiata J. Erikss. \& Hjortstam, in Eriksson, Hjortstam \& Ryvarden, Cortic. N. Eur. (Oslo) 6: 1073, 1981.

Basdiome loosely adnate, effuse, moderately thick, hymenophore grandinioid and with the margin distinctly fibrillose. Hyphal system monomitic, hyphae thin-walled to slightly thick-walled, in $\mathrm{KOH}$ with walls swelling, without clamps. Cystidia terminal, thick-walled except in the apical part, walls swelling or dissolving in $2 \% \mathrm{KOH}$, inamyloid. Basidia subclavate to pedunculate, basally thick-walled, with four sterigmate and without a basal clamp. Basidiospores ellipsoid, thinwalled, smooth, medium-sized (4-6 $\mu \mathrm{m}$ long), walls inamyloid, non-dextrinoid and without cyanophilous reaction.

Remarks - Phanerochaete flabelliradiata agrees in some respects with Hyphodermella, preferably in the shape of the basidia and the nature of the subodontioid hymenium. The main difference from the type species of this genus is the occurrence of cystidia and the morphology of the spores. Another striking feature is the cystidial wall that swells and dissolved partially in $\mathrm{KOH}$. It is considered a synonym of Odonticium Parmasto according to Zmitrovich (2001).

Leifiporia Y.C. Dai, F. Wu \& C.L. Zhao, Mycological Progress 15(7): 802, 2016.

Type species - Leifiporia rhizomorpha Y.C. Dai, F. Wu \& C.L. Zhao, Mycological Progress 15(7): 803, 2016.

Basidiome annual, resupinate, adnate, soft when fresh, brittle when dry, pore surface white to cream. Pores angular; dissepiments thin, entire to slightly lacerate. Hyphal system dimitic, generative hyphae hyaline, thin-walled with clamp connections, frequently branched at right angle; skeletal hyphae present in subiculum only, interwoven, distinctly thinner than generative hyphae, IKI-, CB-; tissues unchanged in $\mathrm{KOH}$. Basidia barrel-shaped to pyriform. Basidiospores ellipsoid, hyaline, thin-walled, smooth, usually bearing one or two guttules, IKI-, CB-.

Remarks - Leifiporia is characterized by an annual growth habit, resupinate basidiocarps with white to cream pore surface, dimitic hyphal system with clamped generative hyphae and branching mostly at right angles, skeletal hyphae present in the subiculum only and distinctly thinner than generative hyphae. Phylogenetic analyses show that Leifiporia belongs to the core polyporoid clade and is closely related to Diplomitoporus overholtsii and Lopharia cinerascens, and then grouped with Pycnoporus and Trametes (Zhao et al. 2016b).

Leiostroma Fr., Novit. F. Suec. part 5 cont. p. 80: 1819.

Type species - Thelephora maculaeformis Fr. Observ. mycol. (Havniae) 1: 150, 1815. (=Thelephora polygonia (Pers.) Pers., Syn. meth. fung. (Göttingen) 2: 574, 1801).

Remarks - A synonym of Peniophora Cooke.

Lentoporia Audet, Mushrooms nomenclatural novelties 5: 1, 2017.

Type species - Poria carbonica Overh., Canadian Journal of Research, Section C 21: 232, 1943.

Remarks - According to Audet (2017e) Lentoporia differs from Antrodia s.str. by only round pores, strongly amyloid hyphae and larger skeletal hyphae, smaller basidia, smaller ellipsoid to short cylindric basidiospores and It differs from Amyloporia s.str. by no yellow basidiome, no bitter taste, and no curved basidiospores.

Lenzitella Ryvarden, Syn. Fung. (Oslo) 5: 174, 1991.

Type species - Lenzitella malenconii Ryvarden, Syn. Fung. (Oslo) 5: 174, 1991.

Remarks - A synonym of Lenzitopsis Malençon \& Bertault.

Lenzitopsis Malençon \& Bertault, Bull. Soc. Mycol. France 79: 82, 1963.

Type species - Lenzitopsis oxycedri Malençon \& Bertault, Bull. Soc. Mycol. France 79: 82, 1963. 
Synonym - Lenzitella Ryvarden.

Basiodiome annual, resupinate to effused-reflexed, submembranaceous, abhymenial surface tomentose to glabrous, hymenial surface irpicoid to irregularly poroid or irregularly lamellate, with brown to black brown. Hyphal system monomitic, hyphae with thin to thickened walls, hyaline to brownish, with clamps. Cystidia absent. Basidia clavate to cylindrical, often somewhat constricted and narrowed towards the base, with 2-4 sterigmata, with a basal clamp. Basidiospores subglobose to broadly ellipsoid, yellowish to brown, warted, thin-walled, IKI-, CB-. It is associated to Juniperus causing a white rot.

Remarks - Lenzitopsis is characterized by the brown lamellate to irpicoid hymenophore and brownish ornamented basidiospores (Malençon \& Bertault 1963, Bernicchia \& Gorjón 2020).

Lepidomyces Jülich, Persoonia 10: 329, 1979.

Type species - Peniophora subcalcea Litsch., Öst. bot. Z. 88: 119, 1939.

Basidiome resupinate, effused, ceraceous-crustaceous, adnate, hymenophore smooth. Hyphal system monomitic, hyphae with clamps, hyaline, thin-walled, indistinct, torulose, Cystidia (leptocystidia) present, hyaline, thin-walled, clamped, basally strongly encrusted. Basidia variable in shape, cylindrical, suburniform or clavate, sometimes pleurobasidia are present, hyaline, with 4sterigmata, and with a basal clamp. Basidiospores cylindrical to narrowly ellipsoid, hyaline, smooth, thin-walled, IKI-, CB-.

Remarks - Lepidomyces is characterized by the basally strongly encrusted leptocystidia and frequent pleurobasidia. The phylogenetic relationships are still unclear, but it may belong to the Pterulaceae in the Agaricales, close to Aphanobasidium (Larsson 2007b).

Leptochaete Lév., Considér. Mycol.: 108, 1846.

Remarks - In the original publication as 'Leptochaete, Lév. = Hymmochaete, Lév.'; nomen rejiciendum.

Leptochaete Zmitr. \& Spirin, Mycena 6: 39, 2006.

Type species - Thelephora sanguinea Fr., Elenchus Fungorum 1: 203, 1828.

Remarks - A homonym of Leptochaete Lév. 1846 and a synonym of Phanerochaete P. Karst.

Leptocorticium Hjortstam \& Ryvarden, Syn. Fung. (Oslo) 15: 23, 2002.

Type species - Corticium cyatheae S. Ito \& S. Imai, Trans. Sapporo nat. Hist. Soc. 16: 143, 1940.

Basidiome resupinate, adnate, crustaceous, hymenophore smooth, margin indeterminate. Hyphal system monomitic, hyphae with clamps, hyaline, thin-walled. Dendrohyphidia present and numerous. Cystidia (leptocystidia), almost subulate, thin-walled, basally encrusted. Basidia clavate, subpedunculate, with 4-sterigmata, and a basal clamp. Basidiospores subfusiform to navicular, smooth, thin- to slightly thick-walled, IKI-, CB-.

Remarks - Leptocorticium reminds in some respects Lepidomyces, but it differs in the terminal basidia and the presence of dendrohyphae. Repetobasidiellum is somewhat similar but it has repetitive basidia (Hjortstam \& Ryvarden 2002c). Phylogenetically its relationships are unclear, but it may belong to the Corticiaceae (Larsson 2007b).

Leptodon Quél., Ench. fung. Eur. (Paris) p. 191, 1886.

Type species - Hydnum pudorinum Fr., Elench. Fung. 1: 133, 1828) (=Hydnum ochraceum Pers. ex J.F. Gmel., in Gmelin, Systema Naturae, 2(2): 1440, 1792).

Remarks - A synonym of Steccherinum S.F. Gray.

Leptosporomyces Jülich, Willdenowia, Beih. 7: 192, 1972.

Type species - Corticium galzinii Bourdot, Rev. Sci. Bourb. Centr. Fr. 23(1): 11, 1910.

Synonym - Fibulomyces Jülich. Includ.: Confertobasidium Jülich. 
Basidiome resupinate, effused, soft and fragile, pellicular, hymenophore smooth, whitish or with some green or pinkish tints. Hyphal system monomitic, hyphae with clamps, thin- to slightly thick-walled, hyaline, encrusted or not. Cystidia usually absent or present as thin-walled hyphoid leptocystidia. Basidia clavate, usually small up to 12-15 $\mu \mathrm{m}$ long, with 4-sterigmata, and a basal clamp. Basidiospores ellipsoid or cylindrical, usually up to $5 \mu \mathrm{m}$ long, smooth, thin-walled, IKI-, CB- (in the generic type) or with a weakly cyanophilous reaction.

Remarks - The generic delimitation of Fibulomyces and Leptosporomyces from Athelia s.l. is somewhat controversial. Both genera were differentiated from Athelia mainly by the smaller size and the shape of basidia (Jülich 1972); Leptosporomyces with Corticium galzinii Bourdot as the generic type species, was differentiated by a more pellicular basidiome with some smaller basidia (up to $12 \mu \mathrm{m}$ long), while the basidiome is membranaceous in Fibulomyces, with Corticium mutabile Bres., as the type species. Molecular data indicate that both Athelia and Athelopsis, are polyphyletic and also seem to support a narrow genus concept close to the one advocated by Jülich (Larsson 2007b). Fibulomyces and Leptosporomyces are phylogenetically very closely related and almost morphologically indistinguishable, for that reason we chose to include all the species in Leptosporomyces instead in Fibulomyces, even if the generic type in Fibulomyces is an older name (page priority is not a criterion when two genera are described in the same publication). However, most of the species have been commonly referred to Leptosporomyces during the recent time (Parmasto et al. 2004), and the use of Fibulomyces needed more nomenclatural proposals. More species are needed to be sequenced to establish a more comprehensible delimitation of these related generic entities. Jülich (1972) erected the genus Confertobasidium to include those athelioid species with pigmented brown basal hyphae, but designating Corticium olivaceoalbum Bourdot \& Galzin, as the generic type, a dimitic species with gloeocystidia and amyloid basidiospores actually placed in Scytinostromella.

Leptoporus Quél., Enchir. fung. (Paris): 175, 1886.

Type species - Boletus mollis Pers., Observ. mycol. (Lipsiae) 1: 22, 1796.

Basidiome annual, sessile to effused reflexed, upper surface white to pale reddish at first, becoming dark reddish purple to purplish brown with age or drying, pore surface becoming purplish brown, pores regular. Hyphal system monomitic, generative hyphae simple-septate. Cystidia or other sterile hymenial elements lacking. Basidia clavate, 4-sterigmate, simple-septate at the base. Basidiospores allantoid, hyaline, negative in Melzer's reagent; causes a brown cubical rot of dead conifers in boreal forests.

Remarks - The genus comes very close to Postia, sharing the same hyphal system, type of spores and brown rot, but is separated because of the simple-septate hyphae and the color change from pinkish white to deep purplish in drying. According to molecular data by Justo et al. (2017), Leptoporus is recovered in the Irpicaceae, close to Ceriporia and Meruliopsis.

Leucogyrophana Pouzar, Česká Mykol. 12: 32, 1958.

Type species - Merulius molluscus Fr., Syst. mycol. (Lundae) 1: 329, 1821.

Basidiome resupinate, effused, easily detachable, membranaceous to pellicular, hymenophore smooth to merulioid, yellowish, orange red to brown coloured. Hyphal system monomitic, hyphae with clamps, more or less branched. Cystidia usually absent. Basidia clavate, with 4-sterigmata, basally clamped. Basidiospores ellipsoid, smooth, thick-walled, usually yellow to brown coloured, dextrinoid, cyanophilous.

Remarks - Leucogyrophana, according to Jarosch \& Besl (2001) and Binder \& Hibbett (2006), is polyphyletic and could be reduced to just the type, Merulius molluscus. It is very closely related to Hygrophoropsis aurantiaca (Wulfen) Maire and included in the Hygrophoropsidaceae, whereas the rest of Leucogyrophana would be placed within Coniophora. According to Jarosch \& Besl l.c., Hydnum pinastri Fr. is included in Hydnomerulius. Leucogyrophana is considered a synonym of Serpula by Zmitrovich \& Spirin (2002), but molecular studies (Jarosch \& Besl l.c., Binder \& Hibbett l.c., Larsson 2007b) seem to indicate that they do not form a monophyletic group. 
Although there are not many morphological characters to support a clear generic separation (larger basidiospores and presence of skeletal hyphae in Serpula), and waiting for further studies, it seems preferably to keep Leucogyrophana and Serpula separate (see also Ginns 1978, 1982).

Leucophellinus Bondartsev \& Singer, in Singer, Mycologia 36(1): 68, 1944.

Type species - Trametes irpicoides Bondartsev ex Pilát, Bull. trimest. Soc. mycol. Fr. 52(3): 311, 1937.

Basidiome resupinate to pileate, pileal surface if present densely tomentose. Pores angular, cream to light brown, context cream. Hyphal system monomitic, generative hyphae simple-septate. Cystidia clavate to tubular, sinuous, thin- to thick-walled, adventitious septa may occur, usually not encrusted but a resinous matter may be covering the cystidia. Basidia clavate, with 4-sterigmate, simple-septate at the base. Basidiospores broadly ellipsoid to ovoid, smooth, thick-walled. Causing a white rot.

Remarks - Leucophellinus is characterized by the simple-septate hyphae, long cystidia, and ellipsoid to ovoid, thick-walled basidiospores. Some similarities share with Oxyporus, but in the latter, cystidia are slender and apically encrusted (Ryvarden 1991).

Licentia Pilát, Ann. Mycol. 38: 66, 1940.

Type species - Licentia yaochanica Pilát, Ann. mycol. 38(1): 66, 1940.

Remarks - A synonym of Lopharia Kalchbr. \& MacOwan.

Licrostroma P.A. Lemke, Can. J. Bot. 42: 762, 1964.

Type species - Corticium subgiganteum Berk., Grevillea 2: 3, 1873.

Basidiome resupinate or effuso-reflexed, confluent, margin determinate, hymenial surface continuous, cream-colored to alutaceous. Hyphal system dimitic, generative hyphae simple-septate, walls thin to partially thickened, basal hyphae aseptate, thick-walled to semisolid, with tapering, antler-like branches (Bovista type), indextrinoid. Pseudocystidia thin to thick-walled elements of deep origin, flexuous-cylindric to subclavate, with rounded or tapering apices, not projecting beyond the level of the hymenium. Basidia scattered, large, cylindric. Basidiospores large, globoid to subgloboid, apiculate, thick-walled, smooth, non-amyloid in Melzer's.

Remarks - Licrostroma is characterized by the simple-septate and binding Bovista-like hyphae, long cylindrical cystidia, and non-amyloid basidiospores. Monotypic genus (Lemke 1964b).

Lilaceophlebia (Parmasto) Spirin \& Zmitr., Nov. sist. Niz. Rast. 37: 177, 2004.

Basionym - Phlebia sect. Lilaceophlebia Parmasto, Consp. System. Corticiac. (Tartu): 94, 1968.

Type species - Corticium lividum Pers., Observ. mycol. (Lipsiae) 1: 38, 1796.

Remarks - A synonym of Phlebia Fr.

Limonomyces Stalpers \& Loer., Can. J. Bot. 60(5): 533, 1982.

Type species - Limonomyces roseipellis Stalpers \& Loer., Can. J. Bot. 60(5): 534, 1982.

Basidiome resupinate, effused, ceraceous. Hyphal system monomitic, hyphae hyaline, with clamps. Basidia urniform, normally originating from thin-walled probasidia, with (1-)2-4 sterigmata. Basidiospores ellipsoid to pip-shaped, smooth, thin-walled, hyaline, inamyloid.

Remarks - Limonomyces is a pathogen pinkish corticoid growing on grasses. Laetisaria also is a pathogen on several herbs, differing in having hyphidia and hyphae lacking clamps. See Stalpers \& Loerakker (1982) for a complete survey on the previous genera.

Lindtneria Pilát, Stud. Bot. Čechoslav. 1: 72, 1938.

Type species - Poria trachyspora Bourdot \& Galzin, Bull. trimest. Soc. mycol. Fr. 41: 219, 1928. 
Basidiome resupinate, effused, membranaceous, hymenophore smooth, merulioid to poroid or hydnoid. Hyphal system monomitic, hyphae with or without clamps (usually both), often inflated where branches occur. Basidia hyaline, clavate to suburniform, with 4-sterigmata, with strongly cyanophilous globules or guttules in the cytoplasma. Basidiospores globose to broadly ellipsoid, hyaline to mostly pale brown, somewhat thick-walled, with an ornamentation of aculei or wing-like crests, IKI-, cyanophilous.

Remarks - Lindtneria is characterized above all by the basidia with cyanophilous granulation and the cyanophilous ornamented basidiospores. It is phylogenetically very closely related to the gastroid genus Stephanospora Pat., sharing common morphological characters as the cyanophilous thick-walled and ornamented basidiospores (Martín et al. 2004). Eriksson \& Ryvarden (1975) pointed a relation of Lindtneria with Cristinia due to the cyanophilous granulations in immature basidia, and this connection is confirmed also by molecular data (Larsson 2007b).

Litschauerella Oberw., Sydowia 19: 43-44, 1966.

Type species - Peniophora abietis (Bourdot \& Galzin) Bourdot \& Galzin, Hyménomyc. de France (Sceaux): 286, 1928.

Basidiome resupinate, effused, adnate, thin, hymenophore smooth, velutinous under the lens, margin thinning out, indeterminate. Hyphal system monomitic, hyphae with clamps, thin-walled. Cystidia (lyocystidia) numerous, narrowly conical with an acute apex, pleural or multi-rooted at the base, thick-walled, heavily encrusted, usually covered with infrequently branched hyphae. Basidia terminal or pleural, short-cylindrical to ellipsoid, with a basal clamp, with 4-sterigmata. Basidiospores globose, ornamented (or smooth), IKI-.

Remarks - Litschauerella is characterized by the multi-rooted lyocystidia covered with branched hyphae and by the ornamented basidiospores. It differs from Tubulicium mainly by the subglobose ornamented spores, vermiform to sigmoid and smooth in the latter. It seems to belong to the Hydnodontaceae, and related to genera such as Brevicellicium, Fibrodontia, Subulicystidium, Trechispora, and Tubulicium (Larsson 2007b).

Lloydella Bres., Lloyd Mycol. Writ. 1: 51, 1901.

Type species - Thelephora cinerascens Schwein., Trans. Am. phil. Soc., Ser. 2 4(2): 167, 1832.

Remarks - A synonym of Lopharia Kalchbr. \& MacOwan.

Lobulicium K.H. Larss. \& Hjortstam, in Hjortstam \& Larsson, Mycotaxon 14: 69, 1982.

Type species - Lobulicium occultum K.H. Larss. \& Hjortstam, in Hjortstam \& Larsson, Mycotaxon 14(1): 70, 1982.

Basidiome resupinate, small, orbicular to effuse, loosely adnate, hymenophore smooth, or more or less blister-like. Hyphal system monomitic, hyphae with clamps, distinct, thin-walled, basal hyphae long-celled, rarely with ampulliform swellings near the septa, subhymenial hyphae short-celled. Cystidia absent. Basidia small, mostly subclavate, usually with 4-sterigmata. Basidiospores with seven lobes, thin-walled, IKI-, CB-.

Remarks - Lobulicium is characterized by the small basidiome growing on very decayed brown-rotted wood and by the lobed basidiospores (Hjortstam \& Larsson 1982). According to molecular data, Lobulicium seems to belong to the Atheliaceae (Larsson 2007b).

Lomatia P. Karst., Bidr. Känned. Finl. Nat. Folk 48: 403, 1889.

Type species - Thelephora salicina Fr., Syst. mycol. (Lundae) 1: 442, 1821.

Remarks - A homonym of Lomatia R. Broome 1819 (Proteaceae).

Lomatina P. Karst., Hedwigia 31: 220, 1892.

Type species - Thelephora salicina Fr., Syst. mycol. (Lundae) 1: 442, 1821.

Remarks - A substitute of Lomatia P. Karst. and a synonym of Cytidia Quél. 
Lopharia Kalchbr. \& MacOwan, Grevillea 10(54): 58, 1881.

Type species - Lopharia lirellosa Kalchbr. \& MacOwan, Grevillea 10(54): 58, 1881 (=Radulum mirabile Berk. \& Broome, J. Linn. Soc., Bot. 14(2): 61, 1875).

Basidiome resupinate, effuse-reflexed to pileate, upper sterile surface tomentose, hymenophore smooth to tuberculate, with irregular ridges to hydnoid or semiporoid, greyish withe to cream or pale ochraceous. Hyphal system dimitic, generative hyphae with clamps, skeletal hyphae thick-walled, hyaline. Cystidia (skeletocystidia) thick-walled, hyaline and encrusted. Basidia clavate, with 4-sterigmata, with a basal clamp. Basidiospores cylindrical to ellipsoid, smooth, hyaline, with distintc oily content, IKI-, CB-.

Remarks - Lopharia differs from Porostereum in the lighter basidiome, hyaline skeletocystidia, and larger basidiospores, usually more than $10 \mu \mathrm{m}$ long, with distinct guttulate content. Hjortstamia differs from both Lopharia and Porostereum in the clampless generative hyphae (Hjorstam \& Ryvarden 1990, Boidin \& Gilles 2002). Lopharia belongs to the Polyporaceae according to molecular data (Larsson 2007b).

Luellia K.H. Larss. \& Hjortstam, Svensk bot. Tidskr. 68: 59, 1974.

Type species - Corticium reconditum H.S. Jacks., Can. J. Res., Section C 26: 154, 1948.

Basidiome resupinate, effused, thin, hymenophore smooth, brown. Hyphal system monomitic, hyphae clamped or not. Cystidia absent. Basidia clavate to pyriform, with 2 or 4sterigmata, with simple basal septum or clamp. Basidiospores fusiform to navicular, smooth, thinwalled, IKI-, CB-

Remarks - Luellia is mainly characterized by its brown basidiome, a rare character in the corticioid fungi, except for the Coniophoraceae; and microscopically by its pyriform basidia and fusiform basidiospores (Larsson \& Hjortstam 1974). Phylogenetically Luellia is included in the Hydnodontaceae although it does not show clear morphological common characteristics with the other members in that family (Larsson 2007b).

Luteoporia F. Wu, Jia J. Chen \& S.H. He, Phytotaxa 263(1): 37, 2016.

Type species - Luteoporia albomarginata F. Wu, Jia J. Chen \& S.H. He, Phytotaxa 263(1): 37, 2016.

Basidiome annual, resupinate, soft when fresh, hard corky upon drying. Hymenophore poroid, pore surface yellow when fresh, straw-colored when dry, pores angular to irregular, dissepiments thin, lacerate, margin cottony, thinning out. Hyphal system monomitic, generative hyphae with clamp connections, become red in $\mathrm{KOH}$. Cystidia-like hyphae projecting out of hymenium, cystidioles present. Basidia subclavate to barrel-shaped, bearing four sterigmata and a basal clamp connection. Basidiospores oblong-ellipsoid, hyaline, thin-walled, smooth, IKI-, CB-. Causing a white rot.

Remarks - Luteoporia is distinguished from other fungal genera by having yellow poroid hymenophore, a monomitic hyphal structure, generative hyphae with clamp connections, cystidia like hyphae projecting out of hymenium (Wu et al. 2016).

Lyoathelia Hjortstam \& Ryvarden, Syn. Fungorum 18: 10, 2004.

Type species - Peniophora laxa Burt, Ann. Mo. Bot. Gard. 12: 224, 1926.

Basidiome resupinate, pellicular to membranaceous, smooth, subiculum thin, white, hyphal chords usually present. Hyphal system monomitic, hyphae thin- to thick-walled, encrusted, all hyphae with clamps. Cystidia capitate, encrusted, thin-walled. Basidia somewhat stalked, thinwalled, with 4-sterigmata, and a basal clamp. Basidiospores ellipsoid, thin-walled or with distinct walls, smooth, IKI-, CB-.

Remarks - Lyoathelia reminds Athelia, but it differs in a more developed basidiome with a thicker subiculum, capitate cystidia, somewhat pedunculate basidia, and almost globose basidiospores (Hjortstam \& Ryvarden 2004a). 
Lyomyces P. Karst., Bidr. Känn. Finl. Nat. Folk 37: 153, 1882.

Type species - Corticium sambuci Pers., Neues Mag. Bot. 1: 111, 1794.

Basidiome resupinate, effused, hymenophore smooth to rarely granular or odontioid. Hyphal system monomitic, all hyphae with clamps, subicular hyphae thin- to thick-walled, usually strongly encrusted. Cystidia (leptocystidia) present, capitate or subulate, usually more or less encrusted. Basidia clavate to suburniform, with 4-sterigmata and a basal clamp. Basidiospores globose, ellipsoid or cylindrical, smooth, thin- to slightly thick-walled, IKI-, with a weak cyanophilous reaction.

Remarks - Lyomyces is separated from Hyphodontia s.l. mainly by the strongly encrusted capitate or subulate cystidia. The relationships with the other Hyphodontia s.l. are not completely clear and more studies are required (see Yurchenko et al. 2017, 2020).

Macrohyporia I. Johans. \& Ryvarden, Trans. Br. mycol. Soc. 72(2): 192, 1979.

Type species - Polyporus dictyoporus Cooke, Grevillea 12(no. 61): 17, 1883.

Basidiome annual to perennial, resupinate, in small patches to widely effused, brittle to hard when dry. Pore surface cream to ochraceous or pale brown, dull, pores 1-5 per mm or larger, context thin. Hyphal system (pseudo)dimitic, generative hyphae simple-septate, thin-walled, binding or strongly branched thick-walled generative hyphae dominating in the trama and context, inamyloid to weakly amyloid, of large diameter. Basidia clavate, 4-sterigmate, simple-septate at the base. Basidiospores hyaline to pale yellow, thin- to slightly thick-walled, subglobose to ellipsoid, non-amyloid. Causing a brown rot.

Remarks - Macrohyporia is characterized by the wide and simple-septate generative hyphae, the wide and tortuous binding hyphae (even in some cases they present simple-septa, so can be considered sclerified generative hyphae), and by the slightly thick-walled subglobose basidiospores (Rajchenberg 1996).

Malacodermum (Fr.) Marchand, Énum. Méth. Rais Fam. Genres Mycophytes (Paris): 200, 1896.

Basionym - Stereum subgen. Malacodermum Fr., Nova Acta R. Soc. Scient. upsal., Ser. 3 1(1): 111, 1851.

Type species - Stereum vitile Fr., K. svenska Vetensk-Akad. Handl., ser. 3: 23, 1848.

Remarks - A name of uncertain application.

Marchandiobasidium Diederich \& Schultheis, in Diederich, Schultheis \& Blackwell, Mycol. Res. 107(5): 524, 2003.

Type species - Marchandiobasidium aurantiacum Diederich \& Schultheis, in Diederich, Schultheis \& Blackwell, Mycol. Res. 107(5): 524, 2003.

Basidiome effused, thin, adnate, granulose, floccose, margin indeterminate, light orange (same colour as sclerotia of Marchandiomyces aurantiacus), hymenium comprising one or several layers of basidia on vertically branching, thin-walled hyphae. Hyphal system monomitic, basal hyphae hyaline, occasionally contorted, thick-walled, subhymenial hyphae hyaline, thin-walled, lacking clamps. Hyphidia, cystidia and other sterile hymenial elements lacking. Basidia initially elongate cylindrical, sometimes with a basal, lateral, ellipsoid probasidial bladder, generally becoming clavate to suburniform, exceptionally with one thin, transverse septum in the upper third, with four long and curved sterigmata, basal clamp lacking. Basidiospores pyriform or lacriform, one side frequently flattened or slightly concave, with a prominent truncate apiculus, hyaline, smooth, thick-walled, nonamyloid, not repetitive.

Remarks - Basidiome of Marchandiobasidium aurantiacum is rare but widespread in Europa, also cited from North America (Diederich \& Lawrey 2007). The anamorph, Marchandiomyces aurantiacus is relatively common and widespread in Europe. The species is a virulent parasite of corticolous Physcia spp., occasionally also attacking neighboring thalli of other lichens, such as Xanthoria spp. The parasite develops mainly in autumn and is able to kill Physcia thalli over larger areas within several weeks. Infection results in the thalli shrinking in thickness, leaving at the end 
the decoloured, film-like, cortical remnants of the hosts. Marchandiobasidium differs from Waitea in the longer basidia, the occasional presence of a basal, lateral probasidial bladder, and dry, nongelatinous sclerotia. Marchandiopsis may be a latter synonym or a closely related genus. The only and type species described in Marchandiopsis differ in the bisterigmatic basidia and thin-walled basidiospores, but it shares the same ecology, parasiting lichens, and it is phylogenetically related. Marchandiobasidium belongs to the Corticiales and it is morphologically and phylogenetically closely related to Erythricium (see also Lawrey et al. 2007).

Marchandiomyces Diederich \& D. Hawksw., in Diederich, Mycotaxon 37: 311, 1990.

Type species - Illosporium corallinum Roberge, in Desmazières, Pl. Crypt. Nord France, Edn 1 32: no. 1551, 1847.

Remarks - A name based on an anamorphic stage. Marchandiomyces aurantiacus (Lasch) Diederich \& Etayo is the anamorph of Marchandiobasidium aurantiacum Diederich \& Schultheis (Diederich \& Lawrey 2007).

Marchandiopsis Ghobad-Nejhad \& Hallenb., Taxon 59(5): 1530, 2010.

Type species - Laeticorticium quercinum J. Erikss. \& Ryvarden, Cort. N Europe 4: 777, 1976.

Basidiome resupinate, closely adnate, orbicular, coocurring with Colpoma quercinum (Pers.) Wallr. from tiny patches around lenticels on bark, rarely confluent, reddish-pink, hymenial surface smooth, gelatinous, margin indistinct. Hyphal system monomitic, hyphae hyaline, thin-walled, simple-septate. Basidia large clavate, developing from bladder-like probasidia, 2-sterigmate. Cystidia absent. Dendrohyphidia few, little branched. Basidiospores large ellipsoid, smooth, thinwalled, with a prominent apiculus, IKI-, CB-.

Remarks - It is characterized by its small reddish-pink gelatinous basidiomes, simple-septate hyphae, bisporic basidia and large basidiospores. It has a peculiar ecology occurring with the lichen Colpoma on Quercus twigs. Phylogenetically it is related to the anamorphic genus Marchandiomyces within the Corticium clade (Ghobad-Nejhad et al. 2010). It seems to be related to the parasitic lichen genus Marchandiobasidium.

Megalocystidium Jülich, Persoonia 10: 139, 1978.

Type species - Corticium leucoxanthum Bres., Fung. trident. 2(11-13): 57, 1898.

Basidiome resupinate, effused, membranaceous to ceraceous, hymenophore smooth, margin byssoid or thinning out. Hyphal system monomitic, hyphae with clamps, hyaline, cylindrical to torulose, more or less thin-walled. Gloeocystidia present, long, flexuous, with oily contents or granular, SA+. Basidia narrowly clavate, hyaline, with 4-sterigmata, a basal clamp present or lacking. Basidiospores cylindrical to ellipsoid, smooth (or minutely ornamented), hyaline, more or less thin-walled, amyloid, CB-.

Remarks - Megalocystidium was described by Jülich (1978b) to include the Gloeocystidiellum luridum and G. lactescens groups proposed by Eriksson \& Ryvarden (1975). The genus thus defined, included species with smooth basidiospores, SA+ gloeocystidia, clamped ( $G$. luridum group) and clampless (G. lactescens group) species. Hjortstam (1987) pointed out that the main features of Thelephora lactescens Berk. were in agreement with those of Gloiothele lamellosa (Henn.) Bres., the type species of Gloiothele, and subsequently regarded as the appropriate genus for the G. lactescens group. Thus, Megalocystidium is including only clamped species in the sense of $\mathrm{Wu}(1996)$.

Megasporia B.K. Cui, Y.C. Dai \& Hai J. Li, in Li \& Cui, Mycologia 105(2): 369, 2013.

Type species - Poria hexagonoides Speg., Anal. Mus. nac. Hist. nat. B. Aires 6: 170, 1898.

Basidiome annual, resupinate. Pore surface white, cream, ochraceous, pale brown, pale purplish brown, lavender tints. Pores mostly angular, rarely round. Hyphal system dimitic with clamped generative hyphae, skeletal hyphae non to strongly dextrinoid and CB-, unbranched to 
sparingly branched. Basidiospores cylindrical to ellipsoid, hyaline, thin-walled, smooth, IKI-, CB-. Polyhedral crystals in subhymenium and hymenium in all species so far. Hyphal pegs absent. Dendrohyphidia in $M$. cavernulosa, difficult to observe. Causing white rot. So far known from the Neotropics.

Remarks - Megasporia can be differentiated from the other similar genera by the acyanophilous hyphae and the neotropical distribution (Li \& Cui 2013).

Megasporoporia Ryvarden \& J.E. Wright, Mycotaxon 16(1): 173, 1982.

Type species - Poria setulosa Henn., Bot. Jb. 28(3): 321, 1900.

Basidiome resupinate, pores generally large, angular to round, pore-surface cream, greyish to pale brown or cinnamon, context usually very thin white to cream or very pale brown. Hyphal system di-trimitic, generative hyphae with clamps, skeletal hyphae thick-walled and dextrinoid, branched vegetative hyphae which may be interpreted as binding hyphae present in most species, dextrinoid, $\mathrm{CB}+$. Cystidia absent, dendrohyphidia present or absent, crystals usually present, often abundantly in the subhymenium and the context. Basidia clavate, with 4-stergimata, with a basal clamp. Basidiospores cylindrical, smooth, thin-walled and large, IKI-. On deciduous wood causing a white rot. Predominantly a tropical genus.

Remarks - Megasporoporia is characterized by its resupinate basidiomes, the large spores and pores besides strongly dextrinoid skeletal hyphae. The presence of dendrohyphidia in some of the species described here seems to point to a relationship to Grammothele as such organs in general are rare among the polypores (Ryvarden et al. 1982). According to Justo et al. (2017), Megasporoporia is classified in the Polyporaceae family.

Megasporoporiella B.K. Cui, Y.C. Dai \& Hai J. Li, in Li \& Cui, Mycologia 105(2): 377, 2013.

Type species - Polyporus cavernulosus Berk., Hooker's J. Bot. Kew Gard. Misc. 8: 235, 1856.

Remarks - A synonym of Megasporia B.K. Cui, Y.C. Dai \& Hai J. Li. There are no morphological or phylogenetical evidence to keep Megasporoporiella separated from Megasporia.

Melzericium Hauerslev, Friesia 10: 316, 1975.

Type species - Corticium udicola Bourdot, Rev. Sci. Bourb. Centr. Fr. 23(1): 10, 1910.

Basidiome annual, resupinate, effused, membranaceous, hymenophore smooth, margin indistinct. Hyphal system monomitic, hyphae with clamps, thin- to slightly thick-walled, often with ampullate septa. Cystidia absent. Basidia clavate, stalked, with (2-)4-sterigmata, and a basal clamp, some pleurobasidia present. Basidiospores ellipsoid to cylindrical, constricted, smooth, thin-walled, hyaline, amyloid.

Remarks - Melzericium is characterized by lacking cystidial elements, the stalked basidia, and by the smooth amyloid basidiospores. The phylogenetic relationships of Melzericium remain still unclear (Larsson 2007b).

Melzerodontia Hjortstam \& Ryvarden, Mycotaxon 12: 177, 1980.

Type species - Melzerodontia aculeata Hjortstam \& Ryvarden, Mycotaxon 12: 178, 1980.

Basidiome resupinate, effuse, adnate, hymenophore grandinioid to distinctly odontioid with rather small aculei. Hyphal system monomitic, all hyphae without clamps, basal hyphae somewhat thick-walled, with a slight dextrinoid reaction, subhymenial hyphae less thick-walled, usually strongly dextrinoid and cyanophilous. Cystidia absent, paraphysoid hyphae or dendrohyphidia may be present. Basidia clavate, with 4-sterigmate, basally thick-walled, dextrinoid, and cyanophilous. Basidiospores subcylindrical, smooth, thin-walled, IKI-, CB-.

Remarks - Melzerodontia is recognized by the aculeate hymenophore, lack of cystidia and clamps, and hyphae and basidial base with a dextrinoid reaction. Monotypic genus of an African species (Hjortstam \& Ryvarden 1980b). 
Membranomyces Jülich, Persoonia 8: 296, 1975.

Type species - Corticium spurium Bourdot, Rev. Sci. Bourb. Centr. Fr. 35: 15, 1922.

Basidiome resupinate, effused, subceraceous to membranaceous, hymenophore smooth. Hyphal system monomitic, hyphae simple-septate, thin- to slightly thick-walled. Cystidia and gloeocystidia absent, hyphoid cystidiols may be present. Basidia clavate, 2-4-spored, simple-septate at the base, guttulate. Basidiospores ellipsoid to subglobose, smooth, thin- or slightly thick-walled, inamyloid, indextrinoid, distinctly guttulate.

Remarks - Membranomyces is characterized by the simple-septate generative hyphae, absence of cystidial elements, and large ellipsoid to subglobose basidiospores. It mainly differs from Clavulicium in the absence of clamps and gloeocystidia. Membranomyces is considered as the sister group of Clavulina cristata which provides another example of a resupinate-clavarioid transformation (Larsson et al. 2004).

Merulicium J. Erikss. \& Ryvarden, Cortic. N. Eur. (Oslo) 4: 859, 1976.

Type species - Merulius fusisporus Romell, Ark. Bot., Ser. 2 2(3): 27, 1911.

Basidiome resupinate, thin, pellicular, hymenophore merulioid when fresh, more or less smooth when dry, margin fibrillose with thin rhizomorphs. Hyphal system dimitic, generative hyphae with clamps, thin-walled, skeletal hyphae thick-walled, dextrinoid. Cystidia (leptocystidia), obclavate, with an obtuse or slightly widened apex, thin-walled. Basidia clavate, with 4-sterigmata and a basal clamp. Basidiospores fusiform, smooth, thin-walled, IKI-, CB-.

Remarks - Merulicium differs from other athelioid species in the dimitic hyphal system with dextrinoid skeletal hyphae (Eriksson \& Ryvarden 1976). Phylogenetically, it belongs to the Pterulaceae in the Agaricales (Larsson 2007b).

Meruliophana Duhem \& Buyck, Cryptogamie, Mycol. 32(2): 136, 2011.

Type species - Meruliophorana mahorensis Duhem \& Buyck, Cryptogamie, Mycol. 32(2): 137, 2011.

Basidiome resupinate, effused, hymenophore tuberculate to merulioid, bluish greyish, more or less translucid, gelatinous to ceraceous, margin indeterminate. Hyphal system monomitic, hyphae with simple-septa, usually thin-walled, more or less widened at septa, not encrusted. Cystidia vesicular or broadly clavate, thin-walled, usually encrusted with irregular crystals. Basidia clavate, stalked, with 4-sterigmata, and a simple basal septum. Basidiospores ellipsoid to cylindrical, smooth, thin-walled, IKI-, guttulate.

Remarks - Meruliophana is characterized by the merulioid bluish-greyish hymenophore, simple-septate hyphae, vesicular cystidia, and smooth not reacting in Melzer's reagent basidiospores (Duhem \& Buyck 2011).

Meruliopsis Bondartsev, in Parmasto, Izv. Akad. Nauk Estonsk. SSR, Ser. Biol. 8: 274, 1959.

Type species - Xylomyzon taxicola Pers., Mycol. Eur. (Erlanga) 2: 32, 1825.

Basidiome annual, resupinate to effused-reflexed, seldom wholly sessile, coriaceousceraceous, upper surface tomentose; white to pale yellow brown. Hymenial surface usually with shallow pits (merulioid), in one species more or less even when young; cream-colored, yellowish orange, red to dark violaceous black. Hyphal system monomitic. Hyphae mostly without clamps, some septa with single or multiple clamps; hyaline. Cystidia present or absent; cylindrical; thinwalled. Basidia subclavate; 4-sterigmate; basal clamp absent. Basidiospores ellipsoid, cylindrical, or allantoid; hyaline; smooth; thin-walled; negative in Melzer's.

Remarks - Meruliopsis Bondartsev (1971), was described to include Xylomyzon taxicola Pers., with a typical poroid hymenophore. Byssomerulius, Candelabrochaete, Ceriporia, Gloeoporus, and Meruliopsis, all sharing generative simple-septate hyphae, are classified by molecular data in the Byssomerulius family, with other clamped species as Phlebia nitidula (P. Karst.) Ryvarden and Ceraceomyces serpens (Tode) Ginns (Larsson 2007b). 
Meruliporia Murrill, Mycologia 34: 596, 1942.

Type species - Merulius incrassatus Berk. \& M.A. Curtis., Hooker's J. Bot. Kew Gard. Misc. 1: 234, 1849.

Remarks - A synonym of Serpula Gray.

Merulius Fr., Syst. mycol. 1: 326, 1821.

Type species - Merulius tremellosus Schrad.: Fr., Syst. mycol. 1: 327, 1821.

Remarks - A synonym of Phlebia Fr.

Metulodontia Parmasto, Consp. System. Corticiac. (Tartu): 117, 1968.

Type species - Kneiffia nivea P. Karst., Hedwigia 35: 173, 1896.

Basidiome resupinate, effused, adnate, hymenophore tuberculate to grandinioid, whitish, margin rhizomorphic. Hyphal system monomitic, hyphae with clamps, thin-walled. Cystidia of two kinds: 1) encrusted cystidia and 2) gloeocystidia, SA+. Basidia clavate, with 4-sterigmata, and a basal clamp. Basidiospores ellipsoid, smooth, thin-walled, IKI-, CB-.

Remarks - Metulodontia is microscopically characterized by the presence of both, encrusted cystidia and sulphopositive gloeocystidia, and smooth inamyloid basidiospores. According to molecular studies, despite the inamyloid basidiospores, belongs to the russuloid clade, as the most basal subclade of the Peniophorales (Larsson \& Larsson 2003, Larsson 2007b).

Metuloidea G. Cunn., Bull. N.Z. Dept. Sci. Industr. Res., Pl. Dis. Div. 164: 250, 263, 1965.

Type species - Trametes tawa G. Cunn., Bull. N.Z. Dept. Sci. Industr. Res., Pl. Dis. Div. 80: 9, 1948.

Basidiome annual, effuse-reflexed to pileate, solitary or imbricate, attached by a lateral base, pilei conchate, applanate, or effused-reflexed, without a cortex, hymenophore hydnoid to poroid with round pores, brown-colored and with a sweet smell. Context of mainly parallel hyphae radiately arranged; hyphal system dimitic to trimitic, generative hyphae branched, septate, with clamp connections, walls thin and hyaline, skeletal hyphae aseptate, unbranched, walls brown; binding hyphae aseptate, freely branched of Bovista type, walls brown. Skeletocystidia or encrusted hyphal ends present in tube mouths, clearly differentiate in old basidiomes. Basidia cylindrical, with 4-sterigmata, and a basal clamp. Basidiospores ellipsoid to short cylindrical, walls smooth, hyaline, nonamyloid.

Remarks - Metuloidea resembles species of Steccherinum which are generally not brown colored and have no sweet smell. Butyrea is phylogenetically closely related, but morphologically it differs in the effused basidiomes without any smell, and in the presence of gloeocystidia and well differentiated skeletocystidia. Phylogenetically, all are phylogenetically classified in the Steccherinaceae family according to Justo et al. (2017).

Michenera Berk. \& M.A. Curtis, J. Linn. Soc., Bot. 10(no. 46): 333, 1868.

Type species - Michenera artocreas Berk. \& M.A. Curtis, J. Linn. Soc., Bot. 10(no. 46): 333, 1868.

Remarks - A name based on an anamorph. Anamorphic state of Licrostroma P.A. Lemke.

Minostroscyta Hjortstam \& Ryvarden, Mycotaxon 79: 194, 2001.

Type species - Minostroscyta discoidalis Hjortstam \& Ryvarden, Mycotaxon 79: 194, 2001.

Basidiome disciform, hymenophore smooth to rough. Hyphal system dimitic, generative hyphae thin-walled, clamped, hyaline, skeletal hyphae thick-walled, moderately branched, cyanophilous, indextrinoid and inamyloid. Gloeocystidia abundant, sinuous, not encrusted, thinwalled, $\mathrm{SV}^{-}$. Basidia clavate, with 4-sterigmate, with a basal clamp. Basidiospores subfusiform, smooth, thin-walled, IKI-, CB-. 
Remarks - Minostroscyta is characterized by the discoid basidiome, dimitic hyphal system with cyanophilous skeletal, large gloeocystidia, and large subfusiform basidiospores (Hjortstam \& Ryvarden 2001).

Mucronella Fr., Hyménomyc. Eur. (Paris): 629, 1874.

Type species - Hydnum calvum Alb. \& Schwein., Consp. fung. (Leipzig): 271, 1805.

Basidiome formed by small aculei, of very small dimension and mostly inconspicuous. Hyphal system monomitic, hyphae simple-septate or with clamps, hyaline. Cystidia absent but hyphoid cystidiols may be present, as well gloeoplerous hyphae. Basidia more or less clavate, with 4-sterigmata. Basidiospores ellipsoid, smooth, thin-walled, hyaline, weakly to distinctly amyloid.

Remarks - Mucronella is macroscopically characterized by the inconspicuous basidiome, formed by small gregarious aculei, microscopically by the absence of cystidial elements and by the amyloid basidiospores. It seems to belong to the Clavariaceae in the Agaricales, but data are not consistent (Larsson 2007b).

Muscinupta Redhead, Lücking \& Lawrey, Mycol. Res. 113(10): 1167, 2009.

Type species - Cantharellus laevis Fr., Syst. mycol. (Lundae) 1: 324, 1821.

Basidiome pileate, dimidiate to flabelliform, with a short stipe but often inconspicuous, hymenophore smooth. Hyphal system monomitic, hyphae without clamps. Cystidia (leptocystidia) present. Basidia clavate, with 4-sterigmata and a simple basal septum. Basidiospores ellipsoid to subglose, smooth, thin-walled, IKI-.

Remarks - Muscinupta is a recently created genus by Lawrey et al. (2009) to include Cantharellus laevis. This species was traditionally included in Cyphellostereum, but Cyphellostereum pusiolum (Berk. \& M.A. Curtis) D.A. Reid, the generic type, differs in some anatomical and morphological features (liquenized structure) and lacks cystidia (Lawrey et al. l.c.). They are not phylogenetically related, Muscinupta laevis belongs to the Hymenochaetales and Cyphellostereum pusiolum to the Agaricales.

Mutatoderma (Parmasto) C.E. Gómez, in Gómez \& Loewenbaum, Bol. Soc. Argent. Bot. 17(3-4): 346, 1976.

Basionym - Hyphoderma sect. Mutatoderma Parmasto, Consp. System. Corticiac. (Tartu): 114, 1968.

Type species - Corticium mutatum Peck, Ann. Rep. N.Y. St. Mus. 43: 23, 1890.

Remarks - Not validly published, basionym not mentioned.

Mycoacia Donk, Medded. Nedl. Mycol. Ver. 18-20: 150, 1931.

Type species - Hydnum fuscoatrum Fr., Novit. fl. Svec. 2: 39, 1814.

Remarks - A synonym of Phlebia Fr. A name largely and still in use for species of Phlebia with odontioid to hydnoid hymenophore. See Nakasone (1997).

Mycoaciella J. Erikss \& Ryvarden, Cort. N Europe 5: 901, 1978.

Type species - Resinicium bisporum Stalpers, Persoonia 9(1): 145, 1976.

Basidiome resupinate, effuse, adnate, not detachable, when fresh of ceraceous consistency, hymenophore hydnoid with dense, cylindrical or narrow conical subulate aculei. Hyphal system dimitic to trimitic, skeletal hyphae straight, parallel, thick-walled, micro-binding hyphae may be present, generative hyphae thin-walled, with clamps, richly branched and interwoven, trama of the aculei to the largest part composed of parallel hyphae, subhymenium thin, distinct, separate from the trama. Cystidia small, cylindrical, thin-walled, with an apical globule of excreted, resinous matter. Basidia clavate, normally with 4-stergimata. Basidiospores narrowly ellipsoid, thin-walled, smooth, inamyloid. 
Remarks - Mycoaciella is characterized by a dimitic to trimitic (with microbinding hyphae), hydnoid hymenophore, and small cystidia with a cap of resinous substance (Eriksson et al. 1978). It was considered a synonym of Phlebia Fr. by Nakasone (2002) but it is still in use by other authors.

Mycobonia Pat. 1894, Bull. Soc. Mycol. Fr. 10(2): 76, 1894.

Type species - Hydnum flavum Berk., Ann. Mg. Nat. Hist. I 10: 380, 1842.

Basidiome pileate, subsessile, dimidiate to reniform, firm and brittle when dry. Hymenial surface with sterile hyphal pegs consisting of thick-walled to almost solid hyphae lacking clamps. Hyphal system dimitic, generative hyphae hyaline, thin-walled, with clamps, binding hyphae hyaline to somewhat yellowish, thick-walled to solid, arboriform. Cystidia and gloeocystidia absent, but tramal cystidia-like present. Basidia clavate, with 4-sterigmate and with a basal clamp. Basidiospores ellipsoid, smooth, thin-walled, IKI-, CB-. On dead wood of hardwoods, causing a white rot.

Remarks - Mycobonia is recognized by the pileate and fragile yellowish basidiomes with numerous hyphal pegs, and microscopically by the arboriform skeletal hyphae (Jülich 1976b, Ryvarden 2010).

Mycoleptodon Pat., Cat. Rais. Pl. Cellul. Tunisie (Paris): 54, 1897.

Type species - Hydnum pudorinum Fr., Elench. fung. (Greifswald) 1: 133, 1828.

Remarks - A synonym of Steccherinum Gray.

Mycoleptodonoides Nikol., Botanicheskie Materialy 8: 117, 1952.

Type species - Mycoleptodonoides vassiljevae Nikol., Botanicheskie Materialy 8: 117, 1952.

Basidiome made up of imbricate pilei, pileus fan-shaped, narrowed into a lateral base or stipe, glabrous, innate-fibrillose, smooth or radiately rugulose, white drying yellow to brown, hymenium covering aculei on the underside of the pileus, concolorous with the pileus. Hyphal system monomitic, generative hyphae inflating in the older parts of the pileus, branched, septate, thin- to thick-walled or even solid, with clamp-connections. Cystidia little differentiate may be present, fusiform to ventricose. Basidia clavate, 4-spored, with a basal clamp. Basidiospores narrowly ellipsoid, more or less curved, smooth, colourless, IKI-, CB-.

Remarks - Mycoleptodonoides is characterized by the flabelliform and stipitate basidiomes with hydnoid hymenophore, monomitic hyphal system, presence of cystidia or cystidiols, and basidiospores with no reaction in Melzer's reagent. It is morphologically closely related to Mycorrhaphium, differentiated in the dimitic hyphal system, and Climacodon which possess gloeocystidia and basidia with a simple basal septum.

Mycolindtneria Rauschert, Feddes Repert. 98: 660, 1987.

Type species - Poria trachyspora Bourdot \& Galzin, Bull. trimest. Soc. mycol. Fr. 41(2): 219, 1925.

Remarks - A superfluous nomen novum for Lindtneria Pilát. Lindtneria Pilát was named after Vojtech H. Lindtner and is not a homonym of Linderia Durand \& Lubber 1889 (Liliaceae).

Mycorrhaphium Maas Geest., Persoonia 2: 394, 1962.

Type species - Hydnum adustum Schwein., Schr. naturf. Ges. Leipzig 1: 103 (1822)

Basidiome sessile to pileate with imbricate growth, flabelliform, narrowed into a lateral base, upper sterile surface glabrous, without concentric zonation, hymenophore hydnoid, white drying yellow to brown. Hyphal system monomitic in the context and dimitic in the aculei, generative hyphae with clamps, thin- to thick-walled, inflated. Gloeocystidia present or absent, cystidiols may be present. Basidia clavate, with 4-sterigmata, with a basal clamp. Basidiospores narrowly ellipsoid, more or less curved, smooth, colourless, IKI-, CB-. 
Remarks - Mycorrhaphium is macroscopically characterized by the flabelliform basidiome with hydnoid hymenophore. The phylogenetic relationships of Mycorrhaphium are unclear but it may belong to the Meruliaceae (Larsson 2007b).

Mycostigma Jülich, Persoonia 8(4): 432, 1976.

Type species - Corticium aegeritoides Bourdot \& Galzin, Bull. Soc. mycol. Fr. 27(2): 249, 1911.

Basidiome consisting of minute globules, separated or aggregated but never confluent, connected by a few hyaline hyphae. Hyphal system monomitic, hyphae indistinct, probably with clamps, hyaline, cylindrical or somewhat torulose, thin-walled. Cystidia or gloeocystidia lacking. Basidia hyaline, clavate, thin-walled, rather small, probably clamped, 4-spored. Basidiospores ellipsoid, thin-walled, smooth, hyaline, inamyloid, CB-.

Remarks - Mycostigma, a monotypic genus, is typified by Corticium aegeritoides, a species characterized by the gregarious basidiome formed by minute globules up to $0.1 \mathrm{~mm}$ in diam., the lack of cystidial elements and the absence of any reaction in Melzer's reagent or cotton blue (Jülich 1976). The phylogenetic relationships of Mycostigma are still unclear.

Mycothele Jülich, Persoonia 8(4): 452, 1976.

Type species - Mycobonia disciformis G. Cunn., Trans. Roy. Soc. N.Z. 83(4): 635, 1956.

Basidiome resupinate to disciform, solitary or in small groups of 3-5, attached by a small central base, membranaceous to coriaceous. Hymenial surface hydnoid with sterile aculei of tramal origin (fascicles arising from the basal layer, traversing the context and projecting to $130 \mu \mathrm{m}, 20-30$ per mm, apices long-acuminate, of cemented hyphae embedding masses of crystals often placed obliquely). Hyphal system monomitic, hyphae hyaline, with clamps, with large masses of crystals in the trama of the aculei. Cystidia and gloeocystidia absent. Basidia stalked clavate, hyaline, with 4-sterigmata and a basal clamp. Basidiospores broadly ellipsoid to subglobose, smooth, hyaline, thin-walled, inamyloid, dextrinoid, CB-.

Remarks - Monotypic genus, only found on Rhopaloslylis sapida, an endemic species of Palmae in New Zealand. Characterized by the small basidiomes and peculiar fascicles. Basidiomes may be disciform and range in diameter from one to a few $\mathrm{mm}$. The surface is pallid sulphur-yellow and densely velutinate with projecting fascicles, in this feature simulating some delicate Hydnum (Jülich 1976).

Myriothele Nakasone, Sydowia 65(1): 104, 2013.

Type species - Epithele philippiae Boidin \& Gilles, Bull. mens. Soc. linn. Lyon 69(9): 195, 2000.

Basidiome resupinate, thick, soft, hymenial surface aculeate from emergent hyphal pegs. Hyphal system monomitic with clamped generative hyphae, subhymenium thickening, a nonagglutinated tissue of upright hyphidia, cystidia and hyphae. Cystidia of three types: obclavate to utriform, often with secondary septa, walls hyaline, thin, smooth; filiform to narrowly obclavate, with a capitate apex, enclosed by a bulbous globule observed in water mounts only; gloeocystidia, rare. Basidia distinctly utriform, clamped at base, 4-sterigmate. Basidiospores broadly ellipsoid to subglobose with hyaline, smooth, weakly cyanophilous, and inamyloid walls that swell in $\mathrm{KOH}$.

Remarks - Epithele philippiae is characterized by hyphal pegs, a thickened, non-agglutinated context, capitate cystidia, utriform basidia, and subglobose basidiospores with walls that swell in $\mathrm{KOH}$. The bulbous globules on the capitate cystidia were not observed in mounts with $\mathrm{KOH}$ or Melzer's reagent. This is a striking species that is clearly not related to Epithele or the Polyporales (Nakasone 2013).

Myxomycidium Massee, Bull. Misc. Inf., Kew: 179, 1901.

Type species - Myxomycidium pendulum Massee, Bull. Misc. Inf., Kew: 180, 1901.

Remarks - A synonym of Mucronella Fr. 
Necator Massee, Bull. Misc. Inf., Kew: 119, 1898.

Type species - Necator decretus Massee, Bull. Misc. Inf., Kew: 119, 1898.

Remarks - A name based on an anamorph. The anamorphic state of Erythricium salmonicolor (Berk. \& Broome) Burds.

Neoantrodia Audet, Mushrooms nomenclatural novelties 6: 1, 2017.

Type species - Polyporus serialis Fr., Syst. mycol. (Lundae) 1: 370, 1821.

Remarks - According to Audet (2017f), Neoantrodia differs from Antrodia s.str. by nonmetachromatic hyphae, smaller not short-lived pedunculate basidia, cystidioles with cap-like crystalline encrustation, and by uninucleate basidiospores.

Neocampanella Nakasone, Hibbett \& Goranova, Botany 87: 877, 2009.

Type species - Dentocorticium blastanos Boidin \& Gilles, Cryptogamie Mycologie 19 (3): 193, 1998.

Basidiome resupinate, effuse, thin, membranous, soft, not gelatinous, smooth to pulverulent, white to pale yellow or orange, margin adnate, more or less abrupt. Hyphal system monomitic, generative hyphae with clamp connections. Dendrohyphidia simple or delicately and finely branched, usually encrusted with tiny hyaline crystals. Cystidia cylindrical, subfusiform to capitate. Basidia ephemeral, more or less cylindrical, bearing 2 or 4 sterigmata. Basidiospores often adherent, ellipsoid or pyriform, often adaxial side slightly flattened, tapering to a prominent, blunt apiculus, with walls thin, hyaline, smooth, IKI-, CB-.

Remarks - Neocampanella is characterized by thin, effuse, nongelatinized basidiomes with dendrohyphidia, cylindrical to capitate cystidia, and ellipsoid to pyriform basidiospores with a distinct, blunt apiculus. Mature basidia are difficult to observe for they probably collapse soon after discharging spores. The characteristic spore shape, slightly tapering toward the prominent apiculus, is reminiscent of basidiospores found in species of Campanella and related genera. Neocampanella, with its corticioid habit and smooth hymenial surface, however, would never be confused with Campanella's pleurotoid form and reticulate lamellae. Dendrohyphidia in the hymenium of Neocampanella may be homologous to the rameal-like structures developed in the pileal epicutis of Campanella and allied genera. The combination of dendrohyphidia and pyriform basidiospores, however, is unique among corticioid fungi to Neocampanella and Brunneocorticium. Neocampanella has a monomitic hyphal system, whereas Brunneocorticium has a dimitic hyphal system with the brown-pigmented skeletal hyphae dominating the context and margin (Nakasone et al. 2009).

Neodatronia B.K. Cui, Hai J. Li \& Y.C. Dai, in Li, Cui \& Dai, Persoonia 32: 177, 2014.

Type species - Neodatronia sinensis B.K. Cui, Hai J. Li \& Y.C. Dai, Persoonia 32: 178, 2014.

Basidiome annual, resupinate, pore surface white, cream to pale brown; pores moderate to small, round to angular; pore surface fragile when dry. Subiculum yellowish brown to cinnamon, corky. Hyphal system dimitic, generative hyphae with clamp connections, skeletal hyphae usually dominating, pale brown to brown, moderately to frequently branched in subiculum and trama, branches of tramal skeletal hyphae usually well differentiated from the main part, IKI-, CB+, tissues darkening in $\mathrm{KOH}$. Dendrohyphidia present in the hymenium and dissepiment edges, cystidia absent, but thin-walled cystidioles usually present. Basidiospores cylindrical, hyaline, thinwalled, smooth, IKI-, CB-. Usually growth on angiosperm wood and causing a white rot.

Remarks - Species of Neodatronia differ from Datronia s.str. by their resupinate basidiomes and moderately to frequently branched skeletal hyphae in subiculum Li et al. (2014).

Neokneiffia Sacc., Tab. compar. Gen. Fung. p. 11, 1898.

Type species - Thelephora setigera Fr., Elench. fung. (Greifswald) 1: 208, 1828.

Remarks - A substitute form Kneiffia Fr. and a synonym of Hyphoderma Wallr. 
Niemelaea Zmitr., Ezhov \& Khimich, Agriculture \& Forestry 61(4): 27, 2015.

Type species - Poria consobrina Bres., Bull. trimest. Soc. mycol. Fr. 41(2): 230, 1925.

Basidiome resupinate, annual, with ceraceous tubular hymenophore. Hyphal system monomitic, hyphae with clamps, of phanerochaetoid appearance, with large oculate clamps and parallel branching pattern, hyaline and loosely arranged in the subiculum, basically inamyloid (seemingly amyloid in the subiculum after heating) and acyanophilous. Cystidia absent. Basidia clavate, with a weak medial constriction, 4-spored, with a large basal clamp. Basidiospores widely ellipsoid, with homogeneous refractive contents, with prominent smooth walls, firm-walled, IKI-, CB-. Causing a white rot.

Remarks - Niemelaea was created for Ceriporiopsis consobrina (Bres.) Ryvarden and Fibuloporia cremea Parmasto by Zmitrovich et al. (2015). The hyphal walls in Niemelaea are a bit thickened, with a phanerochaetoid appearance. Phylogenetically, Niemelaea is phylogenetically classified in the Steccherinaceae family according to Justo et al. (2017).

Nodotia Hjortstam, Mycotaxon 28: 33, 1987.

Type species - Nodotia aspera Hjortstam, Mycotaxon 28(1): 33, 1987.

Basidiome resupinate, effused, hymenophore odontioid, margin indistinct or fibrillose. Hyphal system (pseudo)dimitic, generative hyphae with clamps, thin- to thick-walled, with thickwalled and clamped skeletoid hyphae. Cystidia arising from thick-walled and clamped basal hyphae, encrusted. Basidia clavate, sinuous, with 4-sterigmata, and a basal clamp. Basidiospores subglobose to ellipsoid, smooth, thick-walled, IKI-, CB-.

Remarks - Nodotia was described to include Nodotia aspera, a species close to Hypochnicium but differing in the presence of skeletoid encrusted cystidia (Hjortstam 1987b). Nodotia was later synonymised with Hypochnicium (Hjortstam 1995) but later reintroduced (Hjortstam \& Ryvarden 2004b). Phylogenetically, Nodotia is included among other Hypochnicium species and closely related to the generic type of Hypochnicium (Telleria et al. 2010). Therefore, it can be considered, based on phylogenetic analysis, a synonym of Hypochnicium.

Nodularia Peck, Ann. Rep. N.Y. St. Mus. 24: 96, 1872.

Type species - Nodularia balsamicola Peck, Ann. Rep. N.Y. St. Mus. 24: 96, 1872.

Remarks - A latter homonym of Nodularia Link ex Lyngbye 1819 (Rhodophyceae) and a synonym of Aleurodiscus amorphus (Pers.) J. Schröt.

Nothocorticium Gresl. \& Rajchenb., Mycotaxon 70: 372, 1999.

Type species - Nothocorticium patagonicum Gresl. \& Rajchenb., Mycotaxon 70: 372, 1999.

Basidiome resupinate, orbicular to effused, hymenial surface smooth, velutinate or felty under the lens, pinkish or pale brown coloured, margin fibrillose or forming white hyphae strands developing into the bark. Hyphal system monomitic, hyphae simple-septate, thin- to thick-walled. Hyphidia abundant, filiform, strongly encrusted, simple or with few straight branches. Cystidia absent. Basidia narrowly clavate, large, sinuous, with 2 prominent sterigmata, simple septate at the base. Basidiospores cylindrical or slightly curved, large, smooth, thin-walled, guttulate, IKI-.

Remarks - Nothocorticium is characterized by the orbicular to effused basidioma that develop marginal white strands penetrating into the wood, smooth hymenophore, simple-septate hyphae, large bisterigmatic basidia, large cylindric and thin-walled basidiospores, and encrusted, thickwalled and simple or branched (usually two or few branches) hyphidia. It is a common species and easy to identify, even in the field. It is distributed in the Patagonian Andes of South Sudamerica (Greslebin \& Rajchenberg 1999). Nothocorticium resemble morphologically Corticium, Dendrocorticium, and Marchandiopsis. Preliminary molecular analysis seems to indicate that Nothocorticium is related to Clavulicium macounii (Burt) J. Erikss. \& Boidin ex Parmasto (K-H. Larsson, pers. comm.). 
Obba Miettinen \& Rajchenb., Mycol. Progr. 11(1): 141, 2012.

Type species - Ceriporiopsis rivulosa var. valdiviana Rajchenb., Bol. Soc. Argent. Bot. 30(34): 153, 1995.

Basidiome resupinate, annual to biennial, white when fresh, cream to ochraceous when dry, consistency soft when fresh, drying hard to brittle, subiculum thin, cartilaginous line when dry. Hyphal system monomitic, clamps present, hyphae mostly thin-walled to slightly thick-walled, coarse crystal rosettes in trama and tube mouths, also rhomboidal plates. Subicular hyphae often agglutinated. Cystidia none. Basidia mostly clavate, with 4 sterigmata. Cystidioles subulate to mammiform, common. Basidiospores subglobose to globose, $\mathrm{CB}+$, plasma stained in $\mathrm{CB}$, with a large hyaline oil droplet, thin- to slightly thick-walled, smooth. Associated with white-rot on trunks of gymnosperms and angiosperms.

Remarks - Obba shares a monomitic, clamped hyphal structure with several other polypore genera including Atraporiella, Ceriporiopsis, Erastia, Hapalopilus and Raduliporus. Type species of these genera never show quite the same combination of characters as species of Obba: white, thin, resupinate fruiting bodies, large hymenial cells, abundant cystidioles and subglobose, (very) slightly thick-walled spores. Phylogenetically, Obba is recovered in the 'Cinereomyces clade' with Cinereomyces, Gelatoporia and Sebipora. Species in the 'Cinereomyces clade' share a number of characters: resupinate pale-coloured and poroid fruiting bodies, encrustation in trama or tube mouths, medium-sized spores, the hyaline or shiny oily substance in trama, CRB+ but CB- hyphae, and cultural characters (Miettienen \& Rajchenberg 2012).

Odontia Pers., Neues Mag. Bot. 1: 110, 1794.

Type species - Odontia ferruginea Pers., Neues Mag. Bot. 1: 110, 1794.

Basidiome resupinate, separable from the substratum, arachnoid, continuous, hymenophore granulose to hydnoid, hyphal cords present in subiculum and margins. Hyphal system dimitic, generative hyphae simple-septate or with clamps connections. Cystidia absent. Basidia long clavate or utriform, clamped at base, often sinuous, rarely with transverse septa, 4 sterigmata. Basidiospores bi- and trifurcate, pale brown in $3 \% \mathrm{KOH}$ and in distilled water. Chlamydospores absent. Saprobious, not ectomycorrhizal as in the sister genus Tomentella.

Remarks - Under the name Odontia have been described a heterogeneous group of corticioid species with odontioid to hydnoid hymenophore (eg. species belonging to Dentipellis, Hyphodontia, Hypochnicium, Phlebia, Trechispora, Xylodon, ett.). Odontia ferruginea has long been treated under Tomentella. Phylogenetic analyses of LSU data placed Odontia species as a sister group to Thelephora and Tomentella species. The monophyly of Odontia was strongly supported. In Odontia ectomycorrhizal associations were not observed in nature or in various synthesis experiments (Tedersoo et al. 2014, Yuan et al. 2018).

Odonticium Parmasto, Consp. System. Corticiac. (Tartu): 126, 1968.

Type species - Odonticium romellii (S. Lundell) Parmasto, Consp. System. Corticiac. (Tartu): 126, 1968.

Synonym - Granulocystis Hjortstam.

Basidiome resupinate, effused, membranaceous, hymenophore odontioid to hydnoid, usually cream coloured. Hyphal system monomitic (pseudodimitic), hyphae with simple-septa, thin- to thick-walled, hyaline. Cystidia present or absent, cylindrical, thin- or with distinct walls, hyaline, smooth or encrusted. Basidia cylindrical to clavate, with 4-sterigmata, and a simple basal septum. Basidiospores cylindrical to ellipsoid, smooth, thin-walled, IKI-.

Remarks - Odonticium is characterized by the odontioid to hydnoid hymenophore, monomitic to pseudodimitic hyphal system with simple-septate hyphae, the presence of cystidial elements in most of the species and the smooth inamyloid basidiospores. Phylogenetically it is included in the Rickenella family, but probably Odonticium is a paraphyletic genus (Larsson 2007b). 
Odontina Pat., Hym. Eur. p.147, 1887.

Type species - Hydnum denticulatum Pers., Mycol. eur. (Erlanga) 2: 181, 1825.

Remarks - A synonym of Steccherinum Gray.

Odontiochaete Rick, Ann. mycol. 38(1): 60, 1940.

Type species - Odontiochaete alba Rick, Ann. Mycol. 38(1): 60, 1940.

Remarks - A name of uncertain application (see Hjortstam 1987a).

Odontiopsis Hjortstam \& Ryvarden, Mycotaxon 12: 180, 1980.

Type species - Odontiopsis hyphodontina Hjortstam \& Ryvarden, Mycotaxon 12(1): 180, 1980 (=Odontiopsis ambigua (Berk. \& Broome) Hjortstam, Mycotaxon 28(1): 35, 1987).

Basidiome resupinate, effused, adnate, hymenophore odontioid, aculei with a more or less fimbriate apex. Hyphal system monomitic or subdimitic, all hyphae with clamps, subicular hyphae thick-walled, refractive in $\mathrm{KOH}$ and cotton blue as well in Melzer's reagent, long-celled and with scattered clamps, subhymenial hyphae thin-walled and less refractive, swelling in $\mathrm{KOH}$. Cystidia lacking, hyphal ends numerous in the aculei and in the smooth hymenial surface between the aculei. Basidia clavate, short and with 4-sterigmata. Basidiospores globose, smooth, thin-walled, IKI-.

Remarks - Odontiopsis is recognized by its odontioid hymenophore, projecting hyphal ends, strongly refractive hyphae, shorth and stout basidia, and nearly globose basidiospores (Hjortstam \& Ryvarden 1980b). The phylogenetic relationships are still not resolved (Larsson 2007b).

Odontoefibula C.C. Chen \& Sheng H. Wu, in Chen, Wu \& Chen, MycoKeys 39: 89, 2018.

Type species - Odontoefibula orientalis C.C. Chen \& Sheng H. Wu, in Chen, Wu \& Chen, MycoKeys 39: 90, 2018.

Basidiome annual, resupinate, effused, adnate, membranaceous to ceraceous. Hymenial surface at first honey yellow, becoming ochraceous to pale brown with age, turning dark reddish in $\mathrm{KOH}$, initially smooth to slightly tuberculate, becoming grandinioid to odontioid with age. Aculei conical to cylindrical, separate or fused, up to $0.3 \mathrm{~mm}$ long. Hyphal system monomitic; hyphae normally simple-septate. Subiculum uniform, with dense texture; basal hyphae interwoven, somewhat horizontal or with irregular orientation, colorless, thin- to slightly thick-walled; subicular hyphae somewhat vertical, colorless, thin- to slightly thick-walled. Subhymenium not clearly differentiated from subiculum. Central trama of fairly dense texture; hyphae vertical, colorless, thin- to slightly thick-walled. Cystidia lacking but projecting hyphal ends in the hymenium may be present. Basidia clavate to narrowly clavate, 4-sterigmate. Basidiospores ellipsoid, smooth, thinwalled, inamyloid, non-dextrinoid, CB-.

Remarks - Phaneroites Hjortstam \& Ryvarden, a monotypic genus introduced to accommodate P. subquercinus (Henn.) Hjortstam \& Ryvarden, resembles Odontoefibula in having odontioid hymenial surface and a monomitic hyphal system with ordinarily simple-septate hyphae. However, Phaneroites is distinguished from Odontoefibula by having thin-walled subicular hyphae, a few clamped septa on hyphae next to the substratum and subcapitate cystidia (Hjortstam \& Ryvarden 2010). Moreover, basidiomes of Odontoefibula turn dark reddish in $\mathrm{KOH}$, while this reaction was not reported from Phaneroites.

Oidium Link, in Willdenow, Sp. pl., Ed. 4 6(1): 121, 1824.

Type species - Oidium monilioides (Nees) Link, in Willdenow, Sp. pl., de. 4 6(1): 121, 1824.

Remarks - Some anamorphic states of Botryobasidium Donk were adscribed to Oidium Link. ex Fr. emend. Linder (see Holubová-Jechová 1969). The generic name Oidium was latter accepting to only be conserved for conidials states of Erysiphe R. Hedw. ex DC. and related genera (powdery mildews, Ascomycota). Recently, Braun (2013) proposed to conserve the teleomorph-typified names Blumeria Golovin ex Speer and others against the anamorph-typified name Oidium Link. 
Oligoporus Bref., Unters. Gesammtgeb. Mykol. (Liepzig) 8: 114, 1888.

Type species - Oligoporus farinosus Bref., Unters. Gesammtgeb. Mykol. (Liepzig) 8: 118, 1888 (=Polyporus rennyi Berk. \& Broome, Ann. Mag. nat. Hist., Ser. 4 15(no. 85): 31, 1875).

Basidiomes annual, resupinate to pileate, fleshy when fresh, brittle to hard when dry, mostly white to light colored, sometimes becoming darker by drying. Hymenophore tubular with round pores. Hyphal system monomitic, generative hyphae with clamps, thin- to thick-walled. Cystidia mostly absent, present in few species. Basidia clavate, 4-sterigmate and with a basal clamp. Basidiospores thin-walled, smooth, hyaline, allantoid to ellipsoid, negative in Melzer's reagent. Chlamydospores absent or present. Causing a brown rot, mostly in conifers, more rarely in hardwoods.

Remarks - Oligoporus comprises species causing a brown rot and having a monomitic hyphal system with clamped generative hyphae. Still today, there is controversy with the correct use of Postia or Oligoporus. For nomenclatural comments see Ryvarden (1991), Renvall (1992), Walker (1996), and Bernicchia \& Gorjón (2020). Phylogenetic studies showed that species of both genera appear here and there in the same clade.

Oliveonia Donk, Fungus, Wageningen 28: 20, 1958.

Type species - Sebacina fibrillosa Burt, Ann. Mo. bot. Gdn 13(3): 335, 1926.

Syn.: Sebacinella Hauerslev, Friesia 11: 95, 1977; Monosporonella Oberw. \& Ryvarden, Mycol. Res. 95(3): 378, 1991.

Basidiome resupinate, effused, thin, ceraceous, hymenophore smooth, greyish to white. Hyphal system monomitic, hyphae with or without clamps, thin- to thick-walled in subiculum, often agglutinated, with or without clamps. Cystidia (leptocystidia) present or absent, thin-walled. Basidia clavate to ovoid, with (1-)2-4-sterigmata. Basidiospores subglobose, ellipsoid, citriform, or oblong, smooth, thin-walled, IKI-, CB-, producing secondary spores by repetition.

Remarks - Oliveonia is closely related to Ceratobasidium but originally differing mainly in the presence of leptocystidia, even some acystidiate species have been transferred to Oliveonia. Roberts (1998) referred Oliveonia to the Exidiales.

Oncobasidium P.H.B. Talbot \& Keane, Aust. J. Bot. 19: 203, 1971 1971.

Type species - Oncobasidium theobromae P.H.B. Talbot \& Keane, Aust. J. Bot. 19: 203,

Remarks - A synonym of Thanatephorus Donk.

Osmoporus Singer, Mycologia 36(1): 67, 1944.

Type species - Boletus odoratus Wulfen, in Jacquin, Collnea bot. 2: 150, 1791.

Remarks - Traditionally, the generic type has been treated as Gloeophyllum odoratum (Wulfen) Imazeki. Recently He et al. (2014) proposed a phylogeny to delimit small genera among Gloeophyllum s.str. It differs from Gloeophyllum s.str. in the typically poroid hymenophore and pileate basidiomes.

Oxychaete Miettinen, Mycokeys 17: 19, 2016.

Type species - Polyporus cervinogilvus Jungh., Praemissa in floram cryptogamicam Javae insulae: 45, 1838.

Basidiome effused-reflexed, yellow-brown, light cardboard-like consistency and large, shallow pores. Hyphal system monomitic, hyphae simple-septate, with slightly thick-walled hyphae and abundant subulate, naked, thick-walled cystidia of subhymenial origin. Hymenial branching corymb-like. Cystidia abundant, hymenial, thick-walled, often with an apical crystal cap. Basidia cylindrical to narrowly clavate, collapsing upon spore release and difficult to spot, with 4 sterigmata. Basidiospores cylindrical, curved, thin-walled, smooth, IKI-, plasma stains in CB.

Remarks - Other hydnoid and poroid genera with simple-septate hyphae and encrusted, thickwalled cystidia include Australohydnum, Phlebiopsis, Flavodon and Irpex. The latter two are 
phylogenetically distantly related to Oxychaete, and they possess dimitic hyphal structure quite different from the loose monomitic structure of Oxychaete. Phlebiopsis is phylogenetically distinct from Oxychaete, and its hyphal structure is more compact, even agglutinated (basal layer). Hyphae are also winding and covered with abundant brownish encrustation, which is lacking in Oxychaete. Cystidia are tramal in origin (as opposed to hymenial in Oxychaete). Due to the hyphal structure the basidiome is tougher and not board-like when cut as in Oxychaete (Miettinen et al. 2016).

Oxydontia L.W. Mill., Mycologia 25: 294, 1933.

Type species - Hydnum setosum Pers., Mycol. eur. (Erlanga) 2: 213, 1825.

Remarks - A synonym of Sarcodontia Schulz.

Oxyporus (Bourdot \& Galzin) Donk, Revis. Nederl. Heterobasid. 2: 202, 1933.

Type species - Polyporus connatus connatus Schwein., Trans. Am. phil. Soc., New Series 4(2): 154, 1832 (=Oxyporus populinus (Schumach.) Donk, Meded. Bot. Mus. Herb. Rijks Univ. Utrecht 9: 204, 1933).

Basidiome annual to perennial, resupinate to pileate, fibrous to woody, pilear surface when present white to deep cream, velutinate and often covered with mosses, pore surface white to yellowish, pores mostly small and isodiametric or angular, context white to cream. Hyphal system monomitic to dimitic or pseudodimitic, generative hyphae simple-septate, mostly thick-walled, IKI-. Cystidia obclavate to fusiform, thick-walled, often abundantly encrusted in the apex. Basidia clavate, with 4-sterigmate, with a simple basal septum. Basidiospores globose to broadly ellipsoid, smooth, thin-walled, hyaline, IKI-. Causing a white rot.

Remarks - Oxyporus is characterized by the simple-septate hyphae, presence of encrusted cystidia and more or less globose basidiospores. Rigidoporus is somewhat similar differing in darker orange to brown colored basidiomes and in the mamillated cystidiols. Emmia differs in the strictly monomitic hyphal system with slightly amyloid hyphae (Bernicchia \& Gorjón 2020).

Pachykytospora Kotl. \& Pouzar, Česká Mykol. 17(1): 27, 1963.

Type species - Polyporus tuberculosus Fr., Syst. mycol. (Lundae) 1: 380, 1821.

Remarks - A synonym of Haploporus Singer.

Pachysterigma Johan-Olsen, in Bref., Unters. Gesamt. Mykol. 8: 5, 1889.

Type species - Pachysterigma fugax Johan-Olsen, in Bref., Unters. Gesamt. Mykol. 8: 6, 1889.

Remarks - A synonym of Tulasnella J. Schröt.

Palifer Stalpers \& P.K. Buchanan, New Zealand J. Bot. 29(3): 339, 1991.

Type species - Peniophora verecunda G. Cunn., Trans. Roy. Soc. New Zealand 83: 262, 1955.

Basidiome resupinate, effuse, annual, arachnoid to submembranaceous, hymenophore smooth. Hyphal system monomitic, hyphae with clamps, hyaline, thin- to rarely somewhat thinwalled, locally inflated. Leptocystidia straight, cylindrical, protruding, originating from basal hyphae. Lamprocystidia small, originating in the trama, encrusted with coarse crystals. Capitate cystidia or capitate hyphal ends also present. Basidia subclavate, terminal, hyaline, thin-walled, with 4-sterigmata, with a basal clamp. Basidiospores ellipsoid, hyaline, thin-walled, IKI-.

Remarks - Phylogenetically but also morphologically, it can be considered a synonym of Xylodon. Palifer was erected to include a species described by G.H. Cunningham, Peniophora verecunda from New Zealand with three kinds of cystidia: leptocystidia, lamprocystidia, and capitate cystidia. Later, Hjortstam \& Ryvarden (2007) included three species from Argentina previously referred to Hyphodontia, with odontioid hymenophore and lacking typical leptocystidia (vid. Hyphodontia wrightii Hjortstam \& Ryvarden, $H$. gamundiae Gresl. \& Rajchenb., and $H$. hjortstamii Gresl. \& Rajchenb.). However, Hyphodontia wrightii is a member of Hyphodontia s.str. 
(with typical lagenocystidia), and $H$. gamundiae and $H$. hjortstamii (both with encrusted cystidia) seem to belong in the Xylodon clade, the latter phylogenetically closely related to $P$. verecunda. See also Gorjón (2012). The genus is treated as a synonym of Xylodon (Pers.) Gray, see Viner et al. (2018).

Papyrodiscus D.A. Reid, Beih. Sydowia 8: 333, 1979.

Type species - Papyrodiscus ferrugineus D.A. Reid, Beih. Sydowia 8: 333, 1979.

Basidiome 1.7-4.5 cm diam., attached to very small twigs at the centre of the dorsal surface, and forming flattened circular or slightly pendulous, thin papery fruitbodies. Upper surface feltytomentose, conspicuously zoned and of a uniform bright red-brown colour. Hymenial surface smooth, cream flushed with pink. Hyphal structure monomitic, consisting of hyphae, 3.5-5.5 $\mu \mathrm{m}$ wide, with thin to very slightly brown walls, freely branched and loosely entwined, lack clampconnections at the septa. Cystidia fortuitous. A very thin-walled, hyaline fusiform or lanceolate sterile organs, up to 25(-40) $\mu \mathrm{m}$ long and 4.5-6.0 $\mu \mathrm{m}$ wide may be present. Basidia 12.0-17.0 × 4.5$6.0 \mu \mathrm{m}$, thinwalled, suburniform, with 4 sterigmata. Basidiospores 5.0-6.2 $\times$ 2.0-2.2 $\mu \mathrm{m}$, thinwalled, hyaline, navicular and nonamyloid.

Remarks - Monotypic genus from Papua-New Guinea. According to Reid (1979) is a stereoid fungus apparently showing certain affinities with the polypore genus Flavodon. We have examined the type and the species is similar to a thin papyraceous Stereum or Hymenochaete, with hyphae and hyphal system reminding Hymenochaetaceae, also blackening in $\mathrm{KOH}$, but lacking setal elements.

Parapterulicium Corner, Ann. Bot. London 16: 285, 1952.

Type species - Parapterulicium subarbusculum Corner, Ann. Bot. London 16: 288, 1952.

Remarks - A clavarioid genus belonging to the Varariaceae related to Vararia and Dichostereum (see Leal-Dutra et al. 2018).

Parastereopsis Corner, Nova Hedwigia 27: 331, 1976.

Type species - Parastereopsis borneensis Corner, Nova Hedwigia 27: 331, 1976.

Basidiome infundibuliform, with a hollow stipe, hymenophore smooth to sulcate. Hyphal system monomitic, hyphae with clamps, shorth-celled and inflating. Cystidia absent. Basidia clavate, with (2-)4-sterigmate, and a basal clamp. Basidiospores ellipsoid, smooth, non-amyloid. Conidia globose, smooth, formed in the pileal surface.

Remarks - This genus resembles Stereopsis, but it has a hollow stem or tubaeform basidiome as in Craterellus and inflating short-celled hyphae. Monotypic genus from Borneo (Corner 1976).

Parmastomyces Kotl. \& Pouzar, Reprium nov. Spec. Regni veg. 69: 138, 1964.

Type species - Tyromyces kravtzevianus Bondartsev \& Parmasto, Mycoth. Eston. 1: 22, 1957.

Remarks - A synonym of Sarcoporia P. Karst.

Parvobasidium Jülich, Persoonia 8: 302, 1975.

Type species - Gloeocystidium cretatum Bourdot \& Galzin, Bull. Soc. mycol. Fr. 28(4): 371, 1913.

Basidiome resupinate, effused, adnate, membranaceous, hymenophore smooth creamcoloured. Hyphal system monomitic, hyphae with clamps, hyaline, thin-walled. Gloeocystidia present, hyaline, clavate. Basidia hyaline, small, thin-walled, with a basal clamp. Basidiospores hyaline, small, thin-walled, smooth, inamyloid.

Remarks - Parvobasidium is characterized by the presence of gloeocystidia, the small clavate basidia and the smooth basidiospores with no reaction in Melzer's reagent. It is for the time being included in the Cystosteraceae, a group of gloeocystidiate genera where Crustomyces, Cystidiodontia, Cystostereum, Parvodontia are also present (Larsson 2007b). 
Parvodontia Hjortstam \& Ryvarden, Syn. Fungorum 18: 28, 2004.

Type species - Parvodontia luteocystidiata Hjortstam \& Ryvarden, Syn. Fungorum 18: 28, 2004.

Basidiome resupinate, hymenial surface grandinioid with more or less smooth aculei. Hyphal system monomitic, hyphae smooth, thin-walled, with clamps at all septa. Cystidia abundant, projecting or enclosed, thin-walled, variably in size but with a vesicular shape, encrusted with a yellowish matter, negative in sulphovanilline, cystidiols sinuose, slightly projecting. Basidia subpedunculate, sinuous, thin-walled, with 4-sterigmata, and a basal clamp. Basidiospores narrowly ellipsoid, smooth, thin-walled, IKI-, CB-.

Remarks - Parvodontia is characterized by the grandinioid hymenophore and thin-walled cystidia with yellowish, resinous contents (Hjortstam \& Ryvarden 2004c).

Paullicorticium J. Erikss., Symb. bot. upsal. 16(1): 66, 1958.

Type species - Corticum pearsonii Bourdot, Trans. Br. mycol. Soc. 7(1-2): 52, 1921.

Basidiome resupinate, adnate, thin and inconspicuous, ceraceous when fresh, pruinose when dry, hymenophore more or less porulose. Hyphal system monomitic, hyphae with clamps or simple septa. Cystidia absent. Basidia obconical to pyriform, with (4-)6-8-sterigmata. Basidiospores ellipsoid, allantoid or navicular, smooth, thin-walled, IKI-, CB-.

Remarks - Paullicorticium is characterized by the inconspicuous basidiome and by the obconical to pyriform basidia with usually 6-8-sterigmata. The phylogenetic relationships of Paullicorticium remain still unclear (Larsson 2007b).

Pellicularia Cooke, Grevillea 4(no. 31): 116, 1876

Type species - Pellicularia koleroga Cooke, Grevillea 4(no. 31): 116. 1876.

Remarks - A nomen confusum, see further Donk (1954).

Peniophora Cooke, Grevillea 8: 20, 1879.

Type species - Thelephora quercina Pers., Syn. meth. fung. (Göttingen) 2: 573, 1801.

Synonym - Gloeopeniophora Höhn. \& Litsch.

Basidiome usually resupinate, margin adnate or loosening from the substrata, hymenial surface smooth to tuberculate, grey, violaceous, orange, red or brown. In vertical section, subhyaline to brown, membranaceous to ceraceous, sometimes pseudoparenchymatic. Hyphal system monomitic, hyphae hyaline to brown, thin- to thick-walled with clamps in most species, few with simple-septate hyphae. Dendrohyphidia, gloeocystidia and lamprocystidia present or lacking. Basidia subclavate to cylindrical, with 4-sterigmata, a basal clamp present in most species. Basidiospores usually large, ellipsoid, cylindrical to allantoid, smooth, thin-walled, IKI-, spore print pinkish to reddish.

Remarks - Peniophora is a large genus somewhat heterogeneous but it appears monophyletic in the most analysis (Boidin et al. 1998, Hallenberg et al. 2010, Larsson \& Larsson 2003, Larsson 2007b). The genus Dendrophora differs from Peniophora above all, for the presence of pigmented, thick-walled dendrohyphidia and Duportella for the distinct brown pseudocystidia and the dimitic hyphal system (Boidin 1994). Peniophora is a good example of ecological adaptation to dry and exposed environments. We can find many species with a protective dendrohyphidia layer or more or less coloured hyphae to avoid dehydration or solar radiation, but also large basidiospores to aid the first steps of the germination in a dry substrate. For a complete and recent survey of the genus see Andreasen \& Hallenberg (2009) and Yurchenko (2010).

Peniophorella P. Karst., Bidr. Känn. Finl. Nat. Folk 48: 427, 1889.

Type species - Thelephora pubera Fr., Elench. fung. (Greifswald) 1: 215, 1828.

Basidiome resupinate, effused, adnate, ceraceous to corneous, hymenophore usually smooth to tuberculate, white to yellowish. Hyphal system monomitic, hyphae clamped, subiculum thin and not well differentiate. Cystidia of several kinds, leptocystidia, metuloids or gloeocystidia, 
echinulate cells (echinocysts or stephanocysts) usually present (at least in culture) but often difficult to see. Basidia narrowly to broadly clavate, with 4-sterigmata and basal clamp. Basidiospores cylindrical, ellipsoid or allantoid, smooth, thin-walled, with oily content, abundant in herbarium material, IKI-.

Remarks - Peniophorella differs from Hyphoderma in the presence of echinulate cells, clavate basidia, medium-sized to large basidiospores with oily content, and a bipolar mating system (cf. Larsson 2007a).

Perenniporia Murrill, Mycologia 34(5): 595, 1942.

Type species - Boletus medulla-panis Jacq., Miscell. Austriac. 1: 141, 1778.

Basidiome mostly perennial, rarely annual, resupinate to pileate, pileus smooth, ochraceous to blackish with age. Pore surface white to cream, pores small, isodiametric; context white to pale ochraceous, hard and woody. Hyphal system dimitic (trimitic), generative hyphae thin-walled, hyaline, with clamps, often difficult to observe, skeletal hyphae dominating in the basidiomes, solid to thick-walled, unbranched to moderately branched, non-dextrinoid to strongly dextrinoid, more rarely amyloid in Melzer's reagent. Cystidia none; Basidia clavate, with 4-sterigmate, with a basal clamp. Basidiospores thin- to thick-walled, globose to ellipsoid, drop shaped to truncate, hyaline, non-dextrinoid to strongly dextrinoid in Melzer's reagent, often variable within the same basidiome. On dead and living hardwoods and conifers, causing white rots.

Remarks - Perenniporia is characterized by the poroid basidiomes with ellipsoid to distinctly truncate spores, usually thick-walled and with a variable dextrinoid reaction, combined with a di- to trimitic hyphal system where the vegetative hyphae are dextrinoid to a variable degree (Decock \& Stalpers 2006, Ryvarden 1991, Ryvarden \& Melo 2017, Bernicchia \& Gorjón 2020).

Perplexostereum Ryvarden \& Tutka, Synopsis Fugnorum 32: 72, 2014.

Type species - Stereum endocrocinum Berk., Hooker's J. Bot. Kew Gard. Misc. 6: 169, 1854.

Basidiome perennial, pileate dimidiate, pileus dark brown, zonate, finely velutinate to pubescent. Hymenial surface smooth, ochraceous, in section with a thin black zone between the hymenial part and the upper surface of the pileus. Hyphal system dimitic, generative hyphae hyaline and with clamps, skeletal hyphae pale brown and almost solid. Cystidia present in the hymenium, hyaline tubular, thin-walled. Basidia clavate, with 4-sterigmate, basally clamped. Basidiospores subglobose hyaline, ornamented and strongly amyloid in Melzer's reagent. On coniferous wood.

Remarks - Perplexostereum is a monotypic genus macroscopically identical to Xylobolus subpileatum (Berk.) Boidin by its smooth brown zonate pubescent pileus and smooth hymenophore. However, the cystidia and the ornamented amyloid basidiospores exclude any relationship to that genus. The spores remind one of those seen in Dichostereum, a genus however, where all species have resupinate basidiomes and hyaline cystidia are unknown (Ryvarden \& Tutka 2014).

Phaeophlebia W.B. Cooke, Mycologia 48: 401, 1956.

Type species - Merulius strigosozonatus Schwein., Trans. Am. phil. Soc., Ser. 2 4(2): 160, 1834.

Remarks - A synonym of Punctularia Pat.

Phaeophlebiopsis Floudas \& Hibbett, Fungal Biology 119: 707, 2015.

Type species - Phaeophlebiopsis caribbeana Floudas \& Hibbett, Fungal Biology 119: 707, 2015.

Basidiome resupinate, adnate, smooth, and hard, beige to pale brown to grey-brown and occasionally with purple tints, slightly or extensively cracking, periphery either concolorous or brown with very thin white margin, subiculum beige or brown, no hyphal cords. Hyphal system monomitic, without or very rare clamp connections, thin or thick-walled, occasionally with crystals, 
hyphae usually agglutinated, hyaline and very difficult to separate, reminding Phlebiopsis. Cystidia of metuloid appearance, with heavy encrustation of usually coarse crystals, some with brown colors. Basidia clavate to subcylindrical, thin-walled, with four sterigmata and without basal clamp. Basidiospores smooth, thin-walled, subcylindrical, ellipsoid or ovoid, usually one side flattened and very slightly bent, inamyloid.

Remarks - Phaeophlebiopsis is very similar to Phlebiopsis and their separation is not easy only on morphology. Authors introduced the genus mainly based on the strongly supported phylogenetic separation (Floudas \& Hibbett 2015). The beige to the brown color in the basidiomes and the brown colored cystidia in Phaeophlebiopsis may be a good character to distinguish both genera.

Phaeoradulum Pat., Bull. Soc. mycol. Fr. 16: 178, 1900.

Type species - Phaeoradulum guadelupense Pat., Bull. Soc. mycol. Fr. 16: 178, 1900.

Basidiome resupinate, membranous, context brown and compact, hymenial surface tuberculate to odontioid, brown. Hyphae without clamps. Cystidia cylindrical, thin-walled, 40-60 $\times$ 8-10 $\mu \mathrm{m}$. Basidiospores ovoid, smooth, 10-12 6 $6 \mu \mathrm{m}$, brownish, with a distinct germ pore.

Remarks - Phaeoradulum guadelupense is a species with brown spores and seems to belong in Coniophoraceae (Hjortstam \& Ryvarden 2007). According to the original description it is close to Radulum (however a name of uncertain application) but differing in the brown basidiospores. It would be desirable to examine the generic type to confirm its position among the corticioid fungi.

Phanerochaete P. Karst., Bidr. Känn. Finl. Nat. Folk 48: 426, 1889.

Type species - Thelephora velutina DC., in De Candolle \& Lamarck, Fl. franç., Edn 3 (Paris) 5/6: 33, 1815.

Basidiome resupinate, closely attached to the substrate or commonly more or less detachable, ceraceous when fresh and wet, membranaceous when dried, whitish to yellowish, red-orange or brown, hymenophore smooth, tuberculate, hydnaceous or velutinous due to the projecting cystidia, margin fibrillose, fimbriate or with hyphal cords. Hyphal system monomitic, subicular hyphae mostly $5 \mu \mathrm{m}$ wide or more, as a rule thick-walled, with simple septa or occasional to rare single, double or multiple clamps, straight and parallel with scattered ramifications while the subhymenial hyphae are thin-walled, richly branched and intertwined, without clamps, all hyphae smooth or encrusted to some degree. Cystidia numerous, cylindrical or more or less tapered, usually aseptate, always simple septate at the base, naked or strongly encrusted. Basidia narrowly clavate to cylindrical, hyaline, thin-walled, with a simple basal septum. Basidiospores usually narrowly ellipsoid, allantoid to subglobose, hyaline, smooth, thin-walled, usually with small apiculus, IKI-, CB-.

Remarks - Phanerochaete is a large genus, characterized mainly by the ceraceous basidiome, simple-septate generative hyphae (even single or verticillate clamps may occur in basal hyphae), clavate basidia and smooth inamyloid basidiospores. Phanerochaete, according to several molecular studies, is polyphyletic (Lim 2001, De Koker et al. 2003, Wu et al. 2010). De Koker et al. (2003) pointed out that the core of Phanerochaete group contains the type species $P$. velutina and corresponds more or less to Phanerochaete subgen. Phanerochate as defined by Burdsall (1985); these taxa develop true hymenial cystidia. Some species of Phanerochaete were clearly shown to have closer affinities with other polyporoid lineages. This could indicate that convergent morphology to a phanerochaetoid type has occurred several times. The molecular studies by Wu et al. (2010) confirm the complex delimitation with most of Phanerochaete species dispersed in the phlebioid clade of the Polyporales.

Phanerodontia Hjortstam \& Ryvarden, Syn. Fung. (Oslo) 27: 26, 2010. 2010.

Type species - Phanerodontia dentata Hjortstam \& Ryvarden, Syn. Fung. (Oslo) 27: 27, 
Basidiome resupinate, closely adnate, hymenophore rarely smooth, tuberculate or raduloid to distinctly hydnoid, whitish to pale brown, subiculum thin to moderately thick, pale coloured. Hyphal system monomitic, mostly hyphae with simple-septa, some scattered clamps may be present in the subicular hyphae, thin- to thick-walled in the subiculum, aculeal hyphae usually thinwalled, rarely thickened, parallel and closely arranged in the trama of the aculei. Cystidia few or absent, tubular, hyaline, thin or with thickened walls, smooth or encrusted with granular material. Basidia with 4-sterigmata, simple-septate at the base. Basidiospores ellipsoid, smooth, thin-walled, IKI-, CB-.

Remarks - Phanerodontia was erected to accommodate species formerly placed in Phanerochaete with a typical raduloid to hydnoid hymenophore and thick-walled subicular hyphae. Phaneroites differs morphologically by the absence of cystidia and thin-walled subicular hyphae (Hjortstam \& Ryvarden 2010b).

Phaneroites Hjortstam \& Ryvarden, Syn. Fung. (Oslo) 27: 30, 2010.

Type species - Radulum subquercinum Henn., in Warburg, Monsunia 1: 46, 1899.

Basidiome resupinate, adnate, hymenophore slightly tuberculate or odontioid to hydnoid. Hyphal system monomitic, hyphae with simple-septa but scattered clamps may occur in the subicular hyphae, all hyphae thin-walled. Cystidia absent but subcapitate hyphal ends may be present. Basidia terminal, clavate, with 4-sterigmata and a simple basal septum. Basidiospores ellipsoid or subglobose, smooth, thin-walled, IKI-, CB-.

Remarks - Phaneroites was described to accommodate Radulum subquercinum a species characterized mainly by the odontioid hymenophore and hyphae thin-walled and simple septate. It differs from Phanerodontia by the thin-walled hyphae and absence of cystidia (Hjortstam \& Ryvarden 2010b).

Phellinus Quél., Enchir. fung. (Paris): 172, 1886.

Type species - Boletus igniarius L., Sp. pl. 2: 1176, 1753.

Basidiome perennial, resupinate to pileate, single or imbricate with decurrent pore surface, pileus if present, yellowish, rusty brown, grey to black, tomentose, hispid, glabrous or deeply cracked. Pore surface brownish, pores isodiametric, more rarely irregular and angular and slightly split; context dark reddish brown, umber or yellowish brown, mostly woody, more rarely toughfibrous. Hyphal system dimitic with transitions between thin-walled generative hyphae and this thick-walled skeletal type hyphae, generative hyphae hyaline to pale yellow, narrow, thin-walled, simple-septate, skeletal hyphae yellowish to rusty brown, non-septate or with occasional simple septa, mostly thick-walled and wider than the generative hyphae. Hymenial setae and tramal setae absent or present; setal hyphae absent or present in margin, context or hymenium. Basidia broadly clavate to subglobose, 4-sterigmate, simple-septate at the base. Basidiospores globose to cylindrical, smooth, hyaline to rusty brown, thin- to thick-walled, dextrinoid to negative in Melzer's reagent. On dead or living conifers and hardwoods, causing a white rot.

Remarks - Phellinus is a cosmopolitan large genus of polypores with many species of difficult classification and numerous small genera have been proposed the different species. We include it in this manual in a broad sense, just to cover a large number of resupinate poroid species. For a detailed discussion on nomenclature and taxonomy the reader is referred to specific hymenochaetoid polypore papers and manuals (Bernicchia 2005, Bernicchia \& Gorjón 2020, Fiasson \& Niemelä 1984, Larsen \& Cobb-Poulle 1990, Murrill 1907, Rajchenberg et al. 2015, Ryvarden \& Gilbertson 1994, Ryvarden \& Melo 2017).

Phlebia Fr., Syst. mycol. (Lundae) 1: 426, 1821.

Type species - Phlebia radiata Fr., Syst. mycol. (Lundae) 1: 427, 1821.

Synonyms - Cabalodontia Piątek, Hermanssonia Zmitr., Merulius Fr., Jacksonomyces Jülich, Lilaceophlebia (Parmasto) Spirin \& Zmitr., Mycoacia Donk, Mycoaciella J. Erikss. \& Ryvarden, Stereophlebia Zmitr. 
Basidiome resupinate, rarely pileate, with a subceraceous to subgelatinous consistency when fresh, membranaceous to coriaceous when dry, hymenophore smooth, tuberculate, phlebioid, odontioid, merulioid or poroid. Hyphal system monomitic (rare dimitic), hyphae as a rule clamped (few species with simple-septate generative hyphae), embedded in a gelatinous matrix. Cystidia present or absent. Basidia usually narrowly clavate, arranged in a dense palisade, septa difficult to discern due to the gelatinous matrix. Basidiospores allantoid to ellipsoid, smooth, thin-walled, IKI-, CB-.

Remarks - Phlebia, according to molecular data by Larsson et al. (2004), is closely related to Phlebiella, Mycoacia and Mycoaciella, all these genera share, among other characteristics: basidiome gelatinous to corneous, narrowly clavate basidia arranged in a dense palisade and small inamyloid basidiospores. All those genera form the phlebioid clade with other species of Hyphoderma, Ceriporia, Gloeoporus, Ceraceomyces, and Byssomerulius. The synonymy of Merulius, Mycoacia, and Mycoaciella with Phlebia proposed by Nakasone (Nakasone \& Burdsall, 1984, Nakasone 1997, 2002) seems to be molecularly and morphologically justified and it is adopted here. Mycoaciella is the more deviating genus from Phlebia due to its dimitic hyphal system. Many species of Phlebia developing an odontioid to hydnoid hymenophore, need to add supporting structures in the hymenial configuration, and more or less sclerified generative hyphae are indeed present. Therefore, typical skeletals are not deviating elements if are considered to add strength to a more structured basidiome. Parmasto \& Hallenberg (2000) also preferred to keep Phlebia s.l. undivided. Hermanssonia and Stereophlebia were also recently described by Zmitrovich (2018) to accommodate Phlebia centrifuga P. Karst. and Grandinia tuberculata Berk. \& M.A. Curtis as the generic type species, respectively.

Phlebiella P. Karst., Hedwigia 29: 271, 1890.

Type species - Phlebia vaga Fr., Syst. mycol. (Lundae) 1: 428, 1821.

Basidiome resupinate, effused, adnate, thin, often inconspicuous, hymenophore smooth, ceraceous to subgelatinous, margin fibrillose with hyphal strands. Hyphal system monomitic, hyphae smooth, thin-walled, with clamps or simple-septa, usually agglutinated and difficult to discern. Cystidia absent. Basidia pleural, cylindrical, with 4-sterigmata. Basidiospores warted, thinto slightly thick-walled, non-amyloid.

Remarks - Oberwinkler (1965) described Xenasmatella to include pleurobasidiate corticioid species without cystidial elements and smooth to warted basidiospores (with Corticium subflavidogriseum Litsch. as the generic type species), including in the subg. Xenasmatella those species with inamyloid basidiospores and in the subg. Amyloxenasma species with amyloid basidiospores. In the same contribution, he also erected the Xenasmataceae family including the next genera: Acanthobasidium, Xenosperma, Xenasmatella, Litschauerella, and Xenasma. Later, Oberwinkler (1977) considered that Corticium subflavidogriseum could be separated from Phlebia vaga (generic type of Phlebiella) only at specific (not generic) level, rejecting the genus Xenasmatella and re-establishing Phlebiella for species with warted basidiospores. Hjortstam (1983) preferred to keep separated Xenasmatella for the species with 'basidiospores apparently pale brown in the microscope $(\mathrm{KOH})$ and showing a dextrinoid reaction (pale yellowish)'. Phlebiella in a wide old sense included species with smooth and ornamented amyloid or inamyloid basidiospores. Jülich (1979) erected the genus Aphanobasidium for amyloid or inamyloid, smooth spored species. Hjortstam \& Larsson (1987) divided Phlebiella into three subgenera: subg. Phlebiella, with ornamented basidiospores; subg. Aphanobasidium, with smooth and inamyloid spores and subg. Amyloxenasma, with smooth and amyloid basidiospores. Boidin \& Gilles (1989) accepted that arrangement and Hjortstam \& Ryvarden (2005) raised Amyloxenasma to a generic level. In the molecular study by Larsson (2007b), Phlebiella does not show clear relationships with other genera, Amyloxenasma joints other amyloid-spored species in the family Amylocorticiaceae Jülich and Aphanobasidium appears in the family Perulaceae Corner next to, among others, Radulomyces. Relationships between Phlebiella and Radulomyces have also been recently evaluated by Ghobad-Nejhad \& Kotiranta (2007). 
Phlebiopsis Jülich, Persoonia 10: 137, 1978.

Type species - Thelephora gigantea Fr., Observ. mycol. (Havniae) 1: 152, 1815.

Basidiome annual, resupinate, effused, membranaceous to subceraceous, hymenophore smooth to odontioid. Hyphal system monomitic, hyphae simple septate, hyaline, compactly arranged, basal hyphae thick-walled, subiculum well developed. Cystidia hyaline, thick-walled, encrusted. Basidia narrowly clavate, with 4-sterigmata and a simple basal septum. Basidiospores cylindrical to ellipsoid, hyaline, thin-walled, IKI-.

Remarks - Phlebiopsis is closely related to Phanerochaete and both share the simple-septate generative hyphae and the presence of cystidia. Phlebiopsis, as well Scopuloides, are regarded by some mycologists as congeneric with Phanerochaete (Burdsall 1985, Nakasone 1990a), but the phylogeny of this group is somewhat more complex as it was recently shown by Wu et al. (2010). Morphologically, Phlebiopsis differs from Phanerochaete mainly in the subceraceous basidiome, with a thick and dense subiculum with almost indistinctly agglutinate subicular hyphae. Scopuloides differs from the previous genera in the distinct ceraceous basidiome with a dense context and less developed subiculum. Even if these differences seem to be not important and a matter of basidiome configuration as an ecological adaptation, it seems that these genera, although closely related, are phylogenetically more or less separated. See also Phaeophlebiopsis for comparison.

Phlebiporia Jia J. Chen, B.K. Cui \& Y.C. Dai, Mycological Progress 13: 568, 2013.

Type species - Phlebiporia bubalina Jia J. Chen, B.K. Cui \& Y.C. Dai, Mycological Progress 13: 569, 2013.

Basidiome annual, resupinate, hard corky upon drying, hymenophore poroid, pore surface buff to pinkish buff when dry, pores angular to irregular, dissepiments thin, entire to lacerate, margin thin, cream to buff, cottony. Hyphal system monomitic, generative hyphae with simplesepta, dextrinoid, $\mathrm{CB}+$, thick-walled, tissues becoming brownish in $\mathrm{KOH}$. Thin-walled, frequently branched and aseptate quasi-binding hyphae present in the subiculum. Cystidia absent, cystidioles present. Basidia subclavate to barrel shaped, bearing four sterigmata and a basal simple septum. Basidiospores ellipsoid, hyaline, thin-walled, smooth, IKI-, CB-.

Remarks - The genus seems to be related with other species of Phlebia s.l. in the Meruliaceae (Chen \& Cui 2013). It is characterized by the poroid hymenophore, a monomitic hyphal system with simple-septate and dextrinoid hyphae, and smooth, non-reacting in Melzer's reagent basidiospores.

Phlyctibasidium Jülich, Proc. K. Ned. Akad. Wet., Ser. C, Biol. Med. Sci. 77(2): 154, 1974.

Type species - Corticium polyporoideum Berk. \& M.A. Curtis, Grevillea 1(no. 12): 177. 1873.

Remarks - A synonym of Ramaricium J. Erikss.

Physisporinus P. Karst., Bidr. Känn. Finl. Nat. Folk 48: 324, 1889.

Type species - Poria vitrea Pers., Observ. mycol. (Lipsiae) 1: 15, 1796.

Basidiome resupinate, annual, soft to ceraceous, often changing colour on bruising or drying. Pore surface with round pores. Hyphal system monomitic, generative hyphae with clamps or simple septa. Cystidia absent. Basidia clavate, 4-sterigmate, simple-septate or basally clamped. Basidiospores globose to ovoid, smooth, thin-walled, negative in Melzer's reagent. Causing a white rot in rotten wood.

Remarks - Physisporinus presents soft to waxy ceraceous or subgelatinous basidiomes that often change colour when they are bruised or dried, being very fragile when dry, in contrast to Rigidoporus with very hard basidiomes. Also, in Rigidoporus basal hyphae are very thick-walled and can be easily taken as skeletal hyphae. Both genera, have been treated as synonyms by some authors. See also Bernicchia \& Gorjón (2020). 
Physodontia Ryvarden \& H. Solheim, Mycotaxon 6: 375, 1977.

Type species - Physodontia lundellii Ryvarden \& H. Solheim, Mycotaxon 6(2): 376, 1977.

Basidiome resupinate, hymenophore grandinioid, odontioid to hydnoid, porose-reticulate among the aculei, white to pale cream. Hyphal system monomitic, hyphae with clamps, thinwalled. Cystidia of two kind: 1) gloeocystidia oblong, very numerous in the hymenium, subhymenium and trama, 2) leptocystidia present in the hymenium subulate, smooth, hyaline, thinwalled and projecting. Basidia clavate with 4-sterigmata, and with a basal clamp. Basidiospores subglobose, smooth, IKI-, CB-.

Remarks - Physodontia reminds in some respects Trechispora by the consistence of the basidiome and the similar basidia, but the latter lacks gloeocystidia; Cystostereum by the numerous gloeocystidia but differing in the presence of skeletals in the latter; and Gloeocystidiellum s.l. differs clearly in the amyloid basidiospores. Phylogenetically the position of Physodontia is still unclear, but it could belong to the Hymenochaetales (Larsson 2007b).

Physospora Fr., Fl. Scand.: 360, 1835.

Type species - Sporotrichum rubiginosum Fr., Syst. mycol. (Lundae) 3(2): 417, 1832.

Remarks - A name based on an anamorph. Anamorphic state of Botryobasidium Donk.

Pileodon P. Roberts \& Hjortstam, in Hjortstam, Roberts \& Spooner, Kew Bull. 53(4): 817, 1998.

Type species - Pileodon megasporus P. Roberts \& Hjortstam, in Hjortstam, Roberts \& Spooner, Kew Bull. 53(4): 817, 1998.

Basidiome tough, perennial, effuse-reflexed with distinct pileus in the type species, hymenial surface finely odontioid with numerous hyphal pegs often fused in a semi reticulum, brown throughout. Hyphal system dimitic, generative hyphae hyaline, with clamp-connections, skeletal hyphae brown, thick-walled, often nodulose. Cystidia absent, but with brown skeletal hyphae in the hyphal pegs. Basidia large, stalked, with 4 sterigmata. Basidiospores very large, hyaline becoming brown, thin to slightly thick-walled, inamyloid, non-dextrinoid, acynophilous.

Remarks - Pileodon is characterized by a dark brown, effuse-reflexed basidiome, dimitic hyphal system, hyphal pegs, hyphidia, and large basidiospores. For further details see Nakasone (2004). Morphological limits with Veluticeps are uncertain. The hyphal pegs of Pileodon often fuse laterally to form short, thin plates that resemble an extensively developed shallow semi reticulum whereas species in Veluticeps have a smooth to diverse odontioid hymenophore. Also, basidiospores are usually larger ( $>25 \mu \mathrm{m}$ long) than those of Veluticeps. Campylomyces differs in the cupulate or disciform, deeply lobed basidiomes.

Piloderma Jülich, Ber. dt. bot. Ges. 81: 415, 1969.

Type species - Corticium bicolor Peck, Bull. Buffalo Soc. Nat. History 1(2): 62, 1873 (=Sporotrichum fallax Lib., Pl. crypt. Arduenna, fasc. (Liège), 1832).

Basidiome resupinate, effused, loosely adnate, thin, hymenophore smooth to porulose, margin indeterminate, hyphal strands often present. Hyphal system monomitic, hyphae simple-septate, thin-walled, loosely intertwined in the subiculum, smooth or encrusted. Cystidia absent. Basidia clavate, with 2-4-sterigmata, without a basal clamp. Basidiospores ellipsoid to globose, smooth, slightly thick-walled, IKI-.

Remarks - Piloderma is morphologically closely related to Athelia in basidiome structure with the hyphae loosing intertwined but mainly differs in the distinct thick-walled basidiospores (Jülich 1969). Phylogenetical analysis confirms this relationship (Larsson et al. 2004, Larsson 2007b). Piloderma species are reported to be ectomycorrhizal, like other species in Amphinema, Tylospora, etc. (Binder et al. 2005).

Piloporia Niemelä, Karstenia 22(1): 13, 1982.

Type species - Antrodia sajanensis Parmasto, Botanicheskie Materialy 15: 134, 1962. 
Basidiomes pileate, effused-reflexed to resupinate; upper surface tomentose, dark brown; pore surface whitish to cork-colored; tubes concolorous; context duplex with a black line separating the lower cork-colored part from the upper rusty brown part; hyphal system dimitic; generative hyphae with clamps; skeletal hyphae hyaline to brown in upper part of context, finely encrusted in the dissepiments; cystidia none; basidiospores allantoid, hyaline, thin-walled and negative in Melzer's reagent; causing a white rot in conifers and hardwoods.

Remarks - Piloporia is similar to Incrustoporia and Skeletocutis but differs in the brown pigmentation of the hyphae and the duplex structure of the context and subiculum. Phylogenetically, Piloporia is recovered in the Incrustoporiaceae, with Incrustoporia and Skeletocutis as the closest relatives (Justo et al. 2017).

Pirex Hjortstam \& Ryvarden, Mycotaxon 24: 287, 1985.

Type species - Radulum concentricum Cooke \& Ellis, Grevillea 14(no. 69): 13, 1885.

Basidiome resupinate, effused. Hymenophore odontioid or hydnoid to subporoid. Subiculum dark brown. Hyphal system monomitic, hyphae with clamps, subicular hyphae dark coloured. Cystidia absent. Basidia clavate, with 4 sterigmata and a basal clamp. Basidiospores, ellipsoid to subcylindrical, smooth, thin-walled, IKI-, with oily inclusions.

Remarks - Among corticioid genera with aculei and clamped hyphae, Pirex is most similar to Pseudolagarobasidium and Radulodon (Hallenberg et al. 1985). Following Nakasone \& Linder (2012) Radulodon include aculeate species with a ceraceous or cartilaginous texture, mostly agglutinated, hyaline hyphae, with or without hyphidia, and acyanophilous, slightly thick-walled basidiospores containing a resinous globule. In contrast, Pseudolagarobasidium species have a soft to subceraceous, fragile or brittle texture with mostly non-agglutinated, hyaline to light brown hyphae in the aculeus trama and subiculum, tramal cystidia, basidiospores without resinous material, and lack hyphidia. Radulomyces is somewhat similar to the previous genera, however, is distinguished by its ceraceous, hygrophanous basidiome, large basidia and basidiospores, hyphidia, and lack of cystidia. In addition, its hyphae, basidia and basidiospores are filled with resinous matter.

Plicatura Peck, Ann. Rep. Reg. St. N.Y. 24: 75, 1872.

Type species - Plicatura alni Peck, Ann. Rep. N.Y. St. Mus. 24: 75, 1872 (=Merulius niveus Fr., Elench. fung. (Greifswald) 1: 59, 1828).

Syn.: Plicaturopsis D.A. Reid.

Basidiome pileate, more or less cupulate to flabelliform, abhymenial surface velutinous, hymenophore plicate, with bifurcate lamellae, margin involute. Hyphal system monomitic, hyphae with large and conspicuous clamps, thin- to thick-walled, hyaline or brownish in the trama. Cystidia absent. Basidia clavate, with 4-sterigmata and a basal clamp. Basidiospores allantoid, smooth, thinwalled, with a variable amyloid reaction.

Remarks - Plicaturopsis is here treated as a synonym of Plicatura. There are not reliable morphological and molecular characters to keep both genera separated. Plicaturopsis differs from Plicatura in the more folded hymenophore with branched ridges whereas in Plicatura is only irregularly plicate. Both generic type species, Plicaturopsis crispa and Plicatura nivea are also similar in culture (Nakasone 1990a). According to molecular data, it seems to belong to the Amylocorticiaceae (Larsson 2007b).

Plicaturopsis D.A. Reid, Persoonia 3: 150, 1964.

Type species - Cantharellus crispus Pers., Neues Mag. Bot. 1: 106, 1794.

Remarks - A synonym of Plicatura Peck.

Podoscypha Pat., Essai Tax. Hyménomyc. (Lons-le-Saunier): 70, 1900.

Type species - Sterum surinamense Lév., Annls Sci. Nat., Bot., sér. 3 2: 209, 1844. 
Basidiome flabelliform to infundibuliform, pilei fused, more or less stipitate, hymenophore more or less rugose. Hyphal system dimitic, generative hyphae with clamps, skeletal hyphae thickwalled. Cystidia (gloeocystidia) present. Basidia clavate, with 2-4-sterigmata, with a basal clamp. Basidiospores ellipsoid to cylindrical, smooth, thin-walled, hyaline, IKI-.

Remarks - Podoscypha is mainly characterized by the stipitate and flabellate basidiome, the dimitic hyphal system and the presence of gloeocystidia. According to Binder et al. (2005), Podoscypha is phylogenetically related to Abortiporus biennis in the polyporoid clade; in the molecular analysis by Larsson (2007b) it is also placed in the Polyporales and related with other corticioids as Phlebia in the Meruliaceae.

Podoserpula D.A. Reid, Kew Bull. 16(3): 437, 1963.

Type species - Craterellus pusio Berk., in Hooker, Flora Tasman., Fungi 2: 258, 1859.

Basidiome stipitate, simple to ramose, with usually numerous reniform to spathulate pilei, glabrous. Hymenophore longitudinally to irregularly rugose or merulioid. Hyphal system monomitic, hyphae with clamps, hyaline, thin-walled. Cystidia absent. Basidiospores small, ellipsoid to subglobose, smooth, thick-walled, hyaline, hyaline, IKI-.

Remarks - Podoserpula pusio (Berk.) D.A. Reid (the "pagoda fungus") is characterized by the stipitate basidiomes with a merulioid hymenophore. It belongs to the Amylocorticiales (Binder et al. 2010). Another species, Podoserpula miranda B. Buyck, B. Duhem, G. Eyssartier \& M. Ducousso, was described recently (Buyck et al. 2012).

Poria Adans., Fam. Pl. 2: 10, 1763.

Remarks - A nomen dubium as stated by Ryvarden (1991). The name has usually been used for resupinate poroid fungi and several interpretations have been made from the generic concept.

Poriodontia Parmasto, Mycotaxon 14: 103, 1982.

Type species - Poriodontia subvinosa Parmasto, Mycotaxon 14(1): 104, 1982.

Remarks - A synonym of Trichaptum Murrill.

Porogramme (Pat.) Pat., Essai Tax. Hyménomyc. (Lons-le-Saunier): 63, 1900.

Basyonym - Poria subgen. Porogramme Pat., Bull. Soc. mycol. Fr. 15: 199, 1899.

Type species - Poria dussii Pat., Bull. Soc. mycol. Fr. 15: 199, 1899.

Basidiome resupinate, adnate, bluish grey, reddish to almost blackish, pores angular and irregular, in parts labyrinthine or consisting of irregular plates, hymenium restricted to the base of the pores, context dark and resinous hard, old tubes filled with white mycelium, substrate reddened in zones. Hyphal system monomitic, generative hyphae with clamps, first hyaline, later thickwalled and tinted brownish, and then darker in $\mathrm{KOH}$ and dextrinoid, densely intertwined and agglutinated, clamps often difficult to observe. Cystidia and dendrohyphidia absent. Basidiospores ellipsoid, smooth, thin-walled and non-amyloid.

Remarks - The genus is easy to recognize because of the unusual colour and the reddening of the substrate. The hyphal system may be mistaken to be dimitic, but an examination of the thickwalled hyphae demonstrated clamps, thus, they must be interpreted as sclerified generative hyphae. Their reaction in Melzer and in $\mathrm{KOH}$ is quite unique. It may be related to Grammothele where the same type of reaction does occur in the skeletal hyphae and where the same darkening of the fruitbody occurs with age. However, the dendrohyphidia of Grammothele are absent in Porogramme (Ryvarden 1991).

Porostereum Pilát, Bull. Soc. mycol. Fr. 52: 330, 1936.

Type species - Porostereum phellodendri Pilát, Bull. trimest. Soc. mycol. Fr. 52(3): 331, 1937.

Basidiome resupinate, effuse-reflexed to pileate, upper sterile surface tomentose, brown to greyish coloured, hymenophore smooth to tuberculate, usually with brown colours and often with a 
pinkish tint. Hyphal system dimitic, generative hyphae with clamps, skeletal hyphae thick-walled and brown coloured. Cystidia (skeletocystidia or hymenial cystidial) pale brown, thick-walled, smooth or encrusted. Basidia clavate, with 4-sterigmata, with a basal clamp. Basidiospores cylindrical to ellipsoid, smooth, hyaline, IKI-, CB-.

Remarks - We follow Hjortstam \& Ryvarden (1990) and keep Porostereum separated from Lopharia on the base of the brown basidiome, brown metuloids, and smaller spores present in Porostereum. This separation is also supported by molecular data (Yoon et al. 2003). Boidin \& Gilles (2002) described the genus Hjortstamia for the afibulate Porostereum species.

Porotheleum Fr., Observ. mycol. (Havniae) 2: 272, 1818.

Type species - Poria fimbriata Pers., Neues Mag. Bot. 1: 109, 1794.

Basidiome annual, resupinate, effused, hymenophore formed by small cupuliform pores densely aggregated in a stroma, margin rhizomorphic. Hyphal system dimitic, generative hyphae with clamps, skeletal hyphae thick-walled, aseptate. Cystidia absent, cystidiols may be present. Basidia cylindrical, with 4-sterigmata, and a basal clamp. Basidiospores ellipsoid to cylindrical, smooth, thin-walled, IKI-.

Remarks - Porotheleum is characterized by the particular basidiome structure with cupuliform pores embedded in stroma. It has been traditionally treated under the Polyporales, and phylogenetically it is related to other cyphelloid fungi (Bodensteiner et al. 2004).

Porpomyces Jülich, Persoonia 11(4): 425, 1982.

Type species - Poria mucida Pers., Observ. mycol. (Lipsiae) 1: 87, 1796.

Basidiome resupinate, cream-colored or ochraceous, membranaceous, with small, subangular pores. Hyphal system monomitic, hyphae hyaline, thin- to somewhat thick-walled, always with clamps, smooth or the basal ones covered with granules. Cystidia absent. Basidia hyaline, small, distinctly clavate, four-spored. Basidiospores hyaline, broadly ellipsoid, thin-walled, smooth, IKI-, CB-.

Remarks - Poria mucida has usually classified in Ceriporiopsis Domański. Porpomyces is nested in the trechisporoid clade, with other Trechispora species, and other corticioid genera such as Tubulicium and Sistotremastrum (Larsson 2001).

Postia Fr., Hymenomyc. Eur. (Upsaliae): 586, 1874.

Type species - Polyporus lacteus Fr., Syst. mycol. (Lundae) 1: 359, 1821.

Basidiome annual, resupinate to pileate, fleshy when fresh, brittle to hard when dry, mostly white to light coloured, some species changing to bluish or reddish brown when touching, sometimes becoming darker by drying; hyphal system monomitic, generative hyphae with clamps, thin- to thick-walled; cystidia mostly absent, present in a few species; basidia 4-sterigmate and with basal clamp; basidiospores thin- to thick-walled, smooth, hyaline, allantoid to ellipsoid, negative in Melzer's reagent but in some species weakly amyloid in mass, cyanophilous in some species; causes a brown rot, mostly in conifers, more rarely in hardwoods.

Remarks - According to Ryvarden (1991) a nomen provisorium or nudum. See comments in Oligoporus. Oligoporus can be separated from Postia in a more restricted sense, for its cyanophilous and thick-walled basidiospores, contrary to the acyanophilous, thin-walled basidiospores of most species in Postia. However, Oligoporus rennyii, the type species of Oligoporus, received variable bootstrap values according to the analyses by Rajchenberg et al. (2013). Now, we consider all the species under the oldest name of Postia.

Pouzaroporia Vampola, Česká Mykol. 46(1-2): 59, 1992.

Type species - Poria subrufa Ellis \& Dearn., Proc. Roy. Canad. Inst., Ser. Nov. (Ottawa) 1: 89, 1897.

Basidiome annual, resupinate, adnate, soft when fresh, hard and fragile when dried, margin thin and floccose, hymenophore poroid, pore surface whitish to cream when fresh and young, 
reddening at first after bruising. Hyphal system mono- to dimitic, generative hyphae are thinwalled, hyaline, with clamps, skeletal or skeletoid hyphae both thin- and thick-walled, unbranched or only exceptionally branched, present in subiculum and trama, neither amyloid or dextrinoid, terminal parts of hyphae filled with mass of numerous refractive grains. Cystidia and cystidioles absent. Basidia broadly clavate, some of them nearly cylindrical, 4-sterigmatic, hyaline, with a basal clamp. Basidiospores numerous in fresh specimens, elliptical to ovoid, hyaline, smooth, in $\mathrm{KOH}$ mostly with solitary large spherical drop, IKI-. Causing a white rot.

Remarks - Often regarded as a synonym of Ceriporiopsis, the generic name Pouzaroporia was proposed to accommodate Poria subrufa, differing from Ceriporiospsis in the dimitic hyphal system and from Fibroporia in the white type of root (Vampola 1992). Pouzaroporia belongs to Podoscyphaceae D.A. Reid that comprises genera such as Podoscypha, Abortiporus, and Pouzaroporia. Poria subrufa is not related to Poria gilvescens, the generic type of Ceriporiopsis recovered in the Meruliaceae (Justo et al. 2017).

Prototremella Pat., J. Bot. 2: 269, 1888.

Type species - Prototremella tulasnei Pat., J. Bot. 2: 270, 1888.

Remarks - A synonym of Tulasnella J. Schröt.

Pseudochaete T. Wagner \& M. Fisch., Mycol. Progr. 1(1): 100, 2002.

Type species - Auricularia tabacina Sowerby, Brit. Fung., pl. 25, 1797.

Remarks - nom. illegit.; non Pseudochaete W. West \& G.S. West 1903 (Algae).

Pseudofibroporia Yuan Y. Chen \& B.K. Cui, Mycological Progress 16 (5): 527, 2017.

Type species - Pseudofibroporia citrinella Yuan Y. Chen \& B.K. Cui, Mycological Progress 16 (5): 528, 2017.

Basidiome annual, pileate, pileus sessile, flabelliform to dimidiate, fleshy when fresh, crumbly or chalky, and light in weight after drying. Pileal surface white to lemon yellow when fresh, becoming cream to cinnamon buff upon drying. Pore surface lemon yellow to curry buff when fresh, becoming cinnamon buff to clay buff upon drying. Pores angular, dissepiments thin and entire. Context white to lemon yellow, fleshy when fresh, cottony, or corky after drying. Tubes concolorous with pore surface, crumbly or chalky after dying. Hyphal system dimitic in context, monomitic in trama, generative hyphae with clamp connections, skeletal hyphae IKI-, CB-. Cystidia absent but bottle shaped cystidioles occasionally present. Basidiospores are oblong to broadly ellipsoid, hyaline, slightly thick-walled, smooth, IKI-, CB-. Grows on angiosperm wood.

Remarks - Pseudofibroporia is different from Fibroporia in its distinct pileate basidiomes with entire margin and lacking rhizomorphs. For further information and relations with other poroid genera see Chen et al. (2017).

Pseudolagarobasidium J.C. Jang \& T. Chen, Trans. Br. mycol. Soc. 85(2): 374, 1985.

Type species - Pseudolagarobasidium leguminicola J.C. Jang \& T. Chen, Trans. Br. mycol. Soc. 85(2): 374, 1985. (= Hydnum subvinosum Berk. \& Broome, J. Linn. Soc., Bot. 14(2): 60, 1875).

Basidiome resupinate, effuse, adnate, soft, subceraceous, membranaceous, or cretaceous, hymenial surface odontoid, or raduloid, occasionally reticulate to subporoid. Hyphal system monomitic or dimitic, generative hyphae with clamps, hyaline, yellow to light brown, cyanophilous, all microscopical elements often finely encrusted with resinous material (observed in water mounts) that readily dissolves in $\mathrm{KOH}$; microbinding hyphae aseptate, frequently branched, non-staining. Cystidia originating in aculei trama, subiculum, or subhymenium (tramal cystidia) and hymenium (hymenial cystidia), cylindrical, clavate, or subfusiform, clamped at base, contents homogeneous, staining deeply in phloxine and cotton blue, SV-, walls hyaline, thin, cyanophilous. Basidia clavate to cylindrical, sometimes with a median constriction, clamped at base, walls hyaline, thin, 4-sterigmate. Basidiospores globose to ellipsoid with a small, inconspicuous apiculus, 
contents homogeneous, walls hyaline, thin to slightly thickened, smooth, not or weakly cyanophilous, IKI-.

Remarks - Pseudolagarobasidium is distinguished by the soft-textured basidiome, nonagglutinated hyphae throughout the aculei and context (except in one species), the distinct, even, clamped hyphae of the aculei trama, the numerous tramal and hymenial cystidia with homogenous contents that stain deeply in phloxine and cotton blue, and the small, globose to ellipsoid basidiospores. Species of Pseudolagarobasidium are saprobes although several are known to be facultative or opportunistic pathogens or endophytes. See Nakasone \& Linder (2012).

Pseudomerulius Jülich, Persoonia 10: 350, 1979.

Type species - Merulius aureus Fr., Observ. mycol. (Havniae) 1: 101, 1815.

Basidiome resupinate, effused or effuse-reflexed, ceraceous to membranaceous, adnate, hymenophore merulioid, rhizomorphs absent. Hyphal system monomitic, clamps present at all or most septa, some ansiform, hyphae hyaline, thin-walled or somewhat thick-walled and distinctly swelling in $\mathrm{KOH}$. Cystidia absent or only as hyphoid septate-hyphae. Basidia suburniform or narrowly clavate, with 4-sterigmata and a basal clamp. Basidiospores cylindrical or slightly curved, smooth, slightly thick-walled, light yellowish, inamyloid, slightly dextrinoid, cyanophilous.

Remarks - Pseudomerulius is a deviating genus in the corticioid fungi, and phylogenetically it is placed in the Boletales (Larsson et al. 2004, Larsson 2007b, Kotiranta et al. 2011). It is mainly characterized by the open ansiform clamps and by the slightly dextrinoid basidiospores. It is however morphologically similar to Leucogyrophana in the basidiome structure and in the more or less coloured, thick-walled and cyanophilous basidiospores (Eriksson et al. 1981).

Pseudotomentella Svrček, Česká Mykol. 12(2): 67, 1958.

Type species - Hypochnus mucidulus P. Karst., Bidr. Känn. Finl. Nat. Folk 37: 163, 1882.

Basidiome resupinate, separable from the substratum, pelliculose, continuous or discontinuous. Hymenophore smooth, concolorous with or paler than subiculum. Sterile margin indeterminate or determinate. Hyphal cords present in subiculum and margins, mostly dimitic or rarely monomitic. Subicular hyphae usually simple-septate, clamp connections absent to frequent in some species, thin- or thick-walled, hyaline, brownish or partly greenish in KOH. Subhymenial hyphae simple-septate or clamped, thin-walled, hyaline or partly greenish in $\mathrm{KOH}$. Cystidia absent. Basidia utriform (mostly clavate when immature), stalked, sinuous, rarely with transverse septa, hyaline or partly greenish in $\mathrm{KOH}$, 4-sterigmata. Basidiospores lobed, triangular (with widened proximal part) or slightly globose frontal and ellipsoid lateral face, bi- or trifurcate, hyaline or brownish in $3 \% \mathrm{KOH}$ and in distilled water. Chlamydospores present or absent.

Remarks - Pseudotomentella may be distinguished from species of Tomentella from a combination of characters as the basidiospores ornamented with bifurcate aculei, hymenophore smooth, spaheropedunculate basidia, and usually simple-septate hyphae (Svrček 1958, Kõljalg 1996).

Pseudowrightoporia Y.C. Dai, Jia J. Chen \& B.K. Cui, Persoonia 37: 28, 2015. 1982.

Type species - Wrightoporia cylindrospora Ryvarden, Nordic Journal of Botany 2 (2): 147,

Basidiome annual, sessile, pileate, effused-reflexed or re- supinate, soft corky to corky when fresh, corky when dry. Pileal surface beige, pale brown to ochraceous. Pore surface cream, buffyellow, yellowish brown, orange to ochraceous, usually shining; pores large to tiny, round to angular. Context cream, buff, pale orange, ochraceous to brown, corky to cottony. Tubes corky to fibrous-corky. Hyphal system dimitic, generative hyphae with clamp connections, skeletal hyphae dextrinoid or IKI-. Gloeoplerous hyphae present or absent. Gloeocystidia present or absent. Cystidia present or absent. Basidiospores oblong, broadly ellipsoid, ellipsoid to subglobose, hyaline, thin- to thick-walled, finely asperulate, IKI+, $\mathrm{CB}+$ or $\mathrm{CB}-$. Causing a white rot. 
Remarks - Pseudowrightoporia is characterized by soft corky to corky basidiomes when fresh, corky basidiomes when dry, usually with shining pores, corky to fibrous-corky tubes, a dimitic hyphal structure, oblong, broadly ellipsoid, ellipsoid to subglobose, finely asperulate and amyloid basidiospores, and a subtropical to tropical distribution. Pseudowrightoporia differ from Wrightoporia s.str. and other russuloid polypores by poroid basidiomes, more or less yellowish to pale brown pore surface, shining pores, corky to fibrous-corky tubes, and a dimitic hyphal structure with clamped generative hyphae. Based on phylogenetic analyses by Chen et al. (2016) both Wrightoporia cylindrospora and W. japonica were clustered in the Hericiaceae clade that is distant from Wrightoporia lenta, the type species of Wrightoporia.

Pseudoxenasma K.H. Larss. \& Hjortstam, in Hjortstam \& Larsson, Mycotaxon 4: 307, 1976.

Type species - Pseudoxenasma verrucisporum K.H. Larss. \& Hjortstam, Mycotaxon 4(1): 307, 1976.

Basidiome resupinate, effused, thin, hymenophore smooth, ceraceous. Hyphal system monomitic, hyphae with clamps, densely interwoven, indistinct. Cystidia (sulphocystidia) SA+, cylindrical, with apical schizopapillae. Basidia clavate, mostly pleurobasidial, with 4-sterigmata and a basal clamp. Basidiospores broadly ellipsoid to subglobose, verrucose, thick-walled, with a strong amyloid reaction.

Remarks - Pseudoxenasma is easy to recognize by the presence of pleurobasidia, papillate sulphocystidia, and the verrucose amyloid basidiospores (Hjortstam \& Larsson 1976). It seems to belong to the Russulaceae, but its position is still not completely clear (Larsson 2007b).

Pteridomyces Jülich, Persoonia 10(3): 331, 1979.

Type species - Epithele galzinii Bres., Bull. Soc. mycol. Fr. 27(2): 264, 1911.

Basidiome resupinate, effused, adnate, membranaceous, hymenophore minutely odontioid with sterile hyphal pegs. Hyphal system monomitic, hyphae with clamps, hyaline, thin-walled, densely arranged. Hyphal pegs consisting of parallel arranged and hyaline clamped hyphae. Cystidia absent. Basidia clavate, small, hyaline, thin-walled, with 4-sterigmate, with a basal clamp. Basidiospores hyaline, smooth, thin-walled, IKI-, CB-.

Remarks - Pteridomyces was erected to accommodate Epithele galzinii, an inconspicuous species growing on ferns, with a fragile and closely adnate basidiome, hymenophore with sterile hyphal pegs, short basidia, and smooth curved basidiospores (Jülich 1979). Hjortstam (1991) considered Pteridomyces a synonym of Athelopsis emphasizing the similar shape of the basidia and basidiospores. Epitele galzinii shows a quite different structure of the basidiome, not pellicular and the hyphae are not loosely arranged, contrary to Corticium glaucinum Bourdot \& Galzin, the type species of Athelopsis. We prefer to retain Pteridomyces, based on the presence of sterile hyphal pegs and the absence of well a developed subiculum, at least to the moment that phylogenetic relationships of E. galzinii (still not sequenced) with other athelioid genera can be established. The type species of Pteridomyces has exclusively found growing on ferns.

Ptychogaster Corda, Icon. fung. (Prague) 2: 23, 1838.

Type species - Ptychogaster albus Corda, Icon. fung. (Prague) 2: 24, fig. 90, 1838.

Remarks - A poroid species often referred to Postia Fr. or Oligoporus Bref., with an imperfect stage of a brown mass of chlamydospores. Stalpers (2000) considered the species at a generic level.

Pulcherricium Parmasto, Consp. System. Corticiac. (Tartu): 132, 1968.

Type species - Byssus coerulea Lam., Fl. franç. (Paris) 1: (103), 1779.

Remarks - A synonym of Terana Adans.

Punctularia Pat., in Patouillard \& Lagerheim, Bull. Herb. Boissier 3: 57, 1895.

Type species - Corticium tuberculosum Pat., Bull. Soc. mycol. Fr. 8: 118, 1892. 
Basidiome resupinate to effuse-reflexed or dimidiate, abhymenial surface zonate, hymenophore tuberculate or with radial ridges, gelatinous when fresh. Hyphal system monomitic, hyphae with clamps, thin- to thick-walled. Dendrohyphidia present, yellowish to brown. Cystidia absent. Basidia flexuose with an elongated base, with 4-sterigmata and a basal clamp. Basidiospores ellipsoid, smooth, thin-walled, yellowish to brown, IKI-, CB-.

Remarks - Eriksson et al. (1981), basing on the presence of dendrohyphidia and nature of basidia and basidiospores, pointed out a relationship of Punctularia with Laeticorticium (Corticium). Molecular studies confirm this hypothesis and Punctularia is included in the Corticiaceae with other genera such as Corticium, Dendrocorticium, and Vuilleminia (Larsson 2007b).

Punctulariopsis Ghobad-Nejhad, in Ghobad-Nejhad et al. Taxon 59(5): 1529, 2010.

Type species - Vuilleminia subglobispora Hallenb. \& Hjortstam, Mycotaxon 57: 121, 1996.

Basidiome resupinate, closely adnate, subgelatinous when wet, corneous when dry. Hymenial surface smooth, reddish-ochraceous, velutinous to pruinose under the lens. Hyphal system monomitic, all hyphae with clamps, hyphae hyaline and thin-walled in the subhymenium, yellowish brown and thickened in the subiculum. Cystidia absent. Dendrohyphidia hyaline, smooth or irregularly encrusted, sparsely branched. Basidia elongate, large clavate, flexuous, with four sterigmata and a basal clamp. Basidiospores broadly ellipsoid, smooth, with granular contents, moderately thick-walled, IKI-, CB-.

Remarks - Punctulariopsis resemble microscopically to Punctularia, Dendrocorticium, and Vuilleminia. It resembles Punctularia but with thinner basidiome, less developed and hyaline layer of dendrohyphidia, and larger basidia and basidiospores. Dendrocorticium differs in a different basidiome configuration, lacking gelatinous consistency and smaller basidia formed in a distinguishable layer. Vuilleminia is characterized and differentiated mainly by the decorticating habit. Phylogenetically it is related to Punctularia and Dendrocorticium in the Punctulariaceae (Ghobad-Nejhad et al. 2010).

Purpureocorticium Sheng H. Wu, Mycological Progress 17 (3): 360, 2017.

Type species - Purpureocorticium microsporum Sheng H. Wu, Mycological Progress 17 (3): 360. 2017.

Basidiome effused, adnate, membranaceous. Hymenial surface white when fresh, turning purple in $\mathrm{KOH}$, becoming purplish pink or pinkish purple after storage of several years, smooth. Hyphal system monomitic; hyphae nodose-septate. Subiculum fairly uniform, composed of medullary layer, with compact texture; hyphae colourless, interwoven and with irregular orientation, tortuous, indistinct, \pm agglutinated, occasionally irregularly swollen. Hymenial layer not clearly differentiated from subiculum, possibly thickening. Cystidia lacking. Basidia subclavate with a medium constriction, or utriform, 4-sterigmate. Basidiospores ovate-ellipsoid, smooth, thinwalled, often with one oily drop, IKI-, CB-.

Remarks - Purpureocorticium is characterized by having a resupinate basidiome with smooth hymenial surface which turns purple in $\mathrm{KOH}$, and becomes purplish after storage, microscopically having the compact texture of subiculum, nodose-septate thin-walled generative hyphae, absence of cystidia, basidia subclavate with a median constriction, or utriform, bearing ovate-ellipsoid smallsized basidiospores. Purpureocorticium is similar to Phlebia Fr., due to the presence of the dense texture of subiculum, nodose-septate hyphae, and small basidiospores. However, basidia of Phlebia are strictly clavate or subclavate, while those of Purpureocorticium are subclavate with a median constriction or utriform. The phylogenetic analyses by Wu et al. (2017) showed that Purpureocorticium does not belong to the phlebioid clade. Purpureocorticium also resembles Rhizochaete Gresl., Nakasone \& Rajchenb., because hymenophores of both turn violet in $\mathrm{KOH}$, while Rhizochaete differs from the former in having clavate to subcylindrical basidia, and its cystidia contain two types of encrustation. 
Pycnodon Underw., Bull. Torrey Bot. Club 25: 631, 1898.

Type species - Thelephora setigera Fr., Elench. fung. (Greifswald) 1: 208, 1828.

Remarks - A substitute for Kneiffia Fr. and a synonym of Hyphoderma Wallr.

Pycnoporellus Murrill, Bull. Torrey bot. Club 32(9): 489, 1905.

Type species - Polyporus fibrillosus P. Karst., Sydvestra Finlands Polyporeer, Disp. Praes. Akademisk Afhandling (Helsingfors): 30, 1859 (=Hydnum fulgens Fr., Öfvers. K. Svensk. Vetensk.Akad. Förhandl. 9: 130, 1852.

Basidiome annual, pileate or resupinate, when pileate, broadly attached and semicircular or somewhat elongated; pileus tomentose and zonate, bright orange to rust coloured; pore surface orange, pores medium to large, angular; tubes concolorous with pore surface; context orange to orange-buff, soft and fibrous; all tissues deep red in $\mathrm{KOH}$; hyphal system monomitic; generative hyphae thin-walled to thick-walled, mostly encrusted, simple septate; cystidia present in the hymenium, not encrusted, mostly thin-walled, tubular and projecting; spores cylindric to oblongellipsoid, thin-walled, smooth, hyaline, negative in Melzer's reagent. On dead conifers causing a brown rot.

Remarks - Pycnoporellus is easy to identify by the bright orange to reddish-orange colors, and microscopically by the simple-septate hyphae, presence of cystidia. Pycnoporellus is classified in the Polyporales in the 'Antrodia clade' among other genera such as Sparassis, Crustoderma, and Sarcoporia in the Sparassidaceae (Justo et al. 2017).

Raduliporus Spirin \& Zmitr., Mycena 6: 24, 2006.

Type species - Polyporus aneirinus Sommerf., Suppl. Fl. lapp. (Oslo): 273, 1826.

Basidiome annual or persistent, hymenophore poroid, with wide shallow pores, sometimes splitting and becoming irpicoid, soft-corky to membranaceous or ceraceous; trama irregular; context relatively soft, fleshy-ceraceous or membranaceous; hyphal system monomitic; hyphae clamped, thin- to moderately thick-walled. sometimes with verticillate clamps and crystalline encrustation; cystidia absent: basidia clavate-pedunculate, constricted, often long (more than 25 $\mathrm{pm}$ ); basidiospores comma-shaped to broadly ellipsoid, sometimes with medial constriction, faintly cyanophilous.

Remarks - Type species of Ceriporiopsis, C. gilvescens (Bres.) Domański, differs from $P$. aneirinus by narrow suballantoid spores, relatively small basidia, and rod-shaped reddish crystals on basal hyphae. In the phylogeny of Tomšovský et al. (2010), P. aneirinus is recovered in the phlebioid clade with Polyporus resinascens Romell and Poria pseudogilbescens Pilát and more distant C. gilvescens (recently classified in Resiniporus Zmitr.). For now, we consider Ceriporiopsis as a polyphyletic clade, but, according to molecular data, maintaining Raduliporus and Resiniporus as possible synonyms.

Radulochaete Rick, Annls mycol. 38(1): 58, 1940.

Type species - Radulochaete ceracea Rick, Annls mycol. 38(1): 58, 1940.

Remarks - A name of uncertain application. Some species classified by Rick in Radulochaete are transferred to other genera: Radulochaete horridula (Rick) Rick as Hypochnicium horridulum (Rick) Baltazar \& Rajchenb., and Radulochaete flavoalutacea Rick in Hyphodermella corrugata (Fr.) J. Erikss. \& Ryvarden (Baltazar et al. 2016).

Radulodon Ryvarden, Can. J. Bot. 50: 2073, 1972.

Type species - Radulodon americanus Ryvarden, Can. J. Bot. 50(10): 2074, 1972.

Basidiome resupinate, effused, adnate, hymenophore hydnoid, with usually long cylindrical or flattened aculei. Subiculum pale, mainly thin. Hyphal system monomitic, hyphae thin-walled or with a slight thickened wall, all hyphae with clamps. Cystidia lacking or present, thin-walled, more or less tubular. Basidia clavate, with 4-sterigmata and a basal clamp. Basidiospores globose to subglobose, smooth, moderately thick-walled, rarely thin-walled, IKI-, CB-. 
Remarks - Radulodon differs from related genera such as Hyphoderma in its globose thickwalled basidiospores (Ryvarden 1972). From other hydnoid species of Phanerochaete it is distinguished also by the presence of clamped generative hyphae. It is molecularly classified in the Meruliaceae (Larsson 2007b).

Radulodontia Hjortstam \& Ryvarden, Synopsis Fungorum 25: 31, 2008.

Type species - Radulodontia pyriformis, Synopsis Fungorum 25: 32, 2008.

Basidiome resupinate, effused, hymenophore distinctly hydnoid, with aculei often aggregated in small outgrowths, ochraceous coloured. Hyphal system monomitic, hyphae with clamps, hyaline, thin-walled in the trama of the aculei, in the subiculum thickened and abundantly encrusted. Cystidia absent. Basidia clavate, narrowing towards the base, with 4-sterigmata, and a basal clamp. Basidiospores pyriform, smooth, thin-walled, hyaline, IKI-, CB-.

Remarks - Radulodontia is characterized by the distinctly hydnoid hymenophore, absence of cystidial elements, and pyriform basidiospores. The basidiospores remind those of Cylindrobasidium (Hjortstam \& Ryvarden 2008).

Radulomyces M.P. Christ., Dansk bot. Ark. 19: 230, 1960.

Type species - Thelephora confluens Fr., Observ. mycol. (Havniae) 1: 152, 1815.

Synonym - Adustomyces Jülich.

Basidiome resupinate, adnate, effused, ceraceous, hygrophanous, hymenophore smooth, tuberculate, odontioid to raduloid. Hyphal system monomitic, hyphae with clamps, hyaline, thin- to slightly thick-walled. Cystidia absent, hyphal ends frequently present. Basidia clavate, sinuous, with 4-sterigmata and a basal clamp, with abundant oil drops. Basidiospores ellipsoid to globose, smooth or minutely ornamented (spore dimorphism occurs in some species), slightly thick-walled, IKI-, CB-, with abundant oil content.

Remarks - Some controversies exist on the delimitation of the genus. Jülich \& Stalpers (1980) included Radulomyces, Globulicium, Flavophlebia, and Uncobasidium as synonyms of Cerocorticium P. Henn. Cerocorticium is mainly distinguished by large narrowly ellipsoid to subcylindrical thin-walled basidiospores; Globulicium has globose thin-walled basidiospores and a kind of lagenocystidia or encrusted hyphal ends; Flavophlebia is usually well delimited by its yellowish basidiome, ellipsoid thin-walled basidiospores and cylindrical to subfusiform leptocystidia; Uncobasidium differs in the thin-walled basidiospores with apical apiculus, and above all in the characteristical hooked basidia. Jülich \& Stalpers (1980) included Stereum repandum var. lusitanicum Torrend in Cerocorticium although it was later included in the new genus Adustomyces by Jülich (1979) on the basis of the crustaceous basidiome and brownish subicular hyphae. We are of the opinion that these characters can be perfectly consider as an ecological adaptation to dry environments and not a generic deviating element (more or less coloured subicular hyphae can be found among different species in other genera such as Peniophora, Phlebia, Xylobolus). Since molecular studies do not resolve the phylogenetic relationships of Adustomyces (Larsson 2007b) we, following Hjortstam (1998), including Stereum repandum var. lusitanicum in Radulomyces. Molecular data by Hibbett \& Binder (2002) and Larsson et al. (2004) placed Radulomyces in the euagarics clade next to Phlebiella and Coronicium, separated from the rest of the corticioid fungi.

Radulomycetopsis Dhingra, Priyanka \& J. Kaur, Mycotaxon 119: 133, 2012.

Type species - Radulomycetopsis cystidiata Dhingra, Priyanka \& J. Kaur, Mycotaxon 119: 135, 2012.

Basidiome resupinate, adnate, effused, membranous-ceraceous, hymenophore smooth to slightly tuberculate, orange to brownish orange to reddish brown, margin fibrillose, concolorous but paler. Hyphal system monomitic, generative hyphae branched at wide angles, without clamps, basal hyphae covered with a thick sheath of brownish red matter, which dissolves in $3 \% \mathrm{KOH}$. Cystidia projecting, thin- to slightly thick-walled. Hyphidia simple to somewhat branched in the hymenium. 
Basidia clavate to subclavate, 4-sterigmate, without a basal clamp. Basidiospores broadly ellipsoid to subglobose, smooth, thin- to slightly thick-walled, IKI-, CB-. Both basidia and basidiospores rich in oil drops.

Remarks - Radulomycetopsis is similar to Radulomyces differing in the absence of clamp connections and presence of cystidia (Dhingra et al. 2012).

Radulotubus Y.C. Dai, S.H. He \& C.L. Zhao, Nova Hedwigia 103 (1-2): 270, 2016.

Type species - Radulotubus resupinatus Y.C. Dai, S.H. He \& C.L. Zhao, Nova Hedwigia 103 (1-2): 271.

Basidiome annual, resupinate, adnate, waxy to hygrophanous when fresh, becoming soft corky to slightly brittle when dry, hymenophore white to cream when fresh, which become pale brown when bruised, and the bruised part becoming greyish brown to brown when dry. Hyphal system monomitic, generative hyphae hyaline, thin- to thick-walled with clamps, IKI-, CB-. Basidia pleural, pyriform to barrel shaped, with four sterigmata and a basal clamp. Basidiospores hyaline, thin- to slightly thick-walled, smooth, globose, IKI-, CB-. Causing a white rot.

Remarks - Radulotubus is characterized by annual and resupinate basidiomes, white to cream pores when fresh, darkening after bruising and drying, a monomitic hyphal system with clamped generative hyphae, pleural basidia, and globose, hyaline, thin- to slightly thick-walled, smooth, basidiospores without reactions in Melzer's reagent and Cotton Blue. The phylogenetic analyses based on ITS and nLSU sequences showed that Radulotubus belonged to the Pterulaceae clade in Agaricales and was closely related to Aphanobasidium and Radulomyces that included species with smooth to hydnoid hymenophore. Radulotubus is the sole genus with poroid hymenophore in Pterulaceae (Zhao et al. 2016).

Radulum Fr., Syst. orb. veg. (Lundae) 1: 81, 1825.

Type species - Hydnum aterrimum Fr., Syst. mycol. (Lundae) 1: 416, 1821.

Remarks - Radulum, as described by the type is a species belonging to the Diatrypaceae, where traditionally were classified many corticioids with a more or less odontioid to raduloid hymenophore.

Ramaricium J. Erikss., Svensk bot. Tidskr. 48: 189, 1954.

Type species - Ramaricium occultum J. Erikss., Svensk bot. Tidskr. 48(1): 189, 1954.

Basidiome resupinate, effused, hymenophore smooth, ceraceous when fresh, fragile when dry, margin rhizomorphic. Hyphal system monomitic, hyphae with clamps, often with ampullaceous septa, thin- to thick-walled, smooth or encrusted. Hyphidia poorly differentiated, dendrohyphidia present. Cystidia absent. Basidia terminal or pleural, clavate, more or less sinuous, with 4sterigmata and a basal clamp. Basidiospores globose, ellipsoid or cylindrical, smooth or ornamented, thin- to thick-walled, IKI-, cyanophilous.

Remarks - Ramaricium is characterized above all by dendrohyphidia and the cyanophilous, usually warted basidiospores (Eriksson 1954). Ramaricium and the resupinate hydnoid genera Kavinia and Hydnocristella are members of the Gomphales and probably derived from clavarioid forms (Binder et al. 2005, Giachini et al. 2010).

Repetobasidiellum J. Erikss. \& Hjortstam, in Eriksson, Hjortstam \& Ryvarden, Cortic. N. Eur. (Oslo) 6: 1247, 1981.

Type species - Repetobasidiellum fusisporum J. Erikss. \& Hjortstam in Eriksson, Hjortstam \& Ryvarden, Cortic. N. Eur. (Oslo) 6: 1247, 1981.

Basidiome resupinate, adnate, effused, thin, ceraceous to crustaceous, hymenophore smooth, continuous, margin indeterminate. Hyphal system monomitic, hyphae with clamps, thin-walled. Cystidia absent. Dendrohyphidia present. Basidia urniform, with 4-sterigmata, with a basal clamp, produced by internal repetition from old basidia. Basidiospores subfusiform, smooth, thin-walled, IKI-, CB-. 
Remarks - Repetobasidiellum differs from Repetobasidium in the urniform basidia, and in the presence of dendrohyphidia (Eriksson et al. 1981). From a phylogenetical point of view, the relationships of Repetobasidiellum are unclear (Larsson 2007b).

Repetobasidiopsis Dhingra \& Avneet P. Singh, Mycotaxon 97: 115, 2006.

Validated by Repetobasidiopsis Dhingra \& Avneet P. Singh, Mycotaxon 105: 421, 2008.

Type species - Repetobasidiopsis grandisporus, Dhingra \& Avneet P. Singh, Mycotaxon 97: 116, 2006.

Basidiome resupinate, closely adnate effuse, hymenial surface smooth, subceraceous. Hyphal system monomitic, hyphae without clamps, basal hyphae irregularly branched and interwoven into a dense texture, subhymenial hyphae short-celled and compactly packed and appear like pseudoparenchymatous tissue. Cystidia thin-walled, negative to sulfovainillin. Basidia subclavate to suburniform, showing linear repetition, 4-sterigmate. Basidiospores ellipsoid to subfusiform or suballantoid, smooth, thin-walled, IKI-, CB-.

Remarks - Repetobasidiopsis is characterized by the repetitive basidia, absence of clamps, and smooth inamyloid basidiospores. It differs from Repetobasidiellum and Repetobasidium in the absence of clamp connections (Dhingra et al 2006, Dhingra \& Singh Avneet 2008).

Repetobasidium J. Erikss., Symb. bot. upsal. 16(1): 67, 1958.

Type species - Peniophora vilis Bourdot \& Galzin, Hyménomyc. de France (Sceaux): 282, 1928.

Basidiome resupinate, adnate, effused, thin, ceraceous, hymenophore smooth, margin indeterminate. Hyphal system monomitic, hyphae with clamps, thin-walled. Cystidia (leptocystidia) present. Basidia subglobose to pyriform, with 4-sterigmata, with a basal clamp, produced by internal repetition from old basidia. Basidiospores globose to allantoid, smooth, thin-walled, IKI-, CB-.

Remarks - Repetobasidium is characterized above all by the presence of leptocystidia and repetobasida, even the formation of repetitive basidia occurs occasionally in other genera. The phylogenetic position of Repetobasidium and the relationships with other genera is still unclear, but according to some molecular studies it belongs to the Hymenochatales (Larsson et al. 2006, Larsson 2007b).

Resinicium Parmasto, Consp. System. Corticiac. (Tartu): 97, 1968.

Type species - Hydnum bicolor Alb. \& Schwein., Consp. fung. (Leipzig): 270, 1805.

Basidiome, resupinate, effused, adnate, hymenophore smooth, grandinioid, odontioid to hydnoid, membranous to ceraceous, white to yellowish, margin thinning out, indeterminate, pruinose. Hyphal system monomitic, hyphae with clamps (one species with simple septate hyphae is present), thin-walled. Cystidia of two kinds: 1) halocystidia and 2) astrocystidia. Basidia clavate, with 4-sterigmata and a basal clamp. Basidiospores ellipsoid, cylindrical or allantoid, smooth, thinwalled, IKI-, CB-.

Remarks - Resinicium, according to some phylogenetic studies, is a polyphyletic genus, where the circumscription should be restricted to species with asterocystidia and large halocystidia (Larsson 2007b). Skvortzovia is an available genus name for species previously placed in Resinicium lacking typical asterocystidia.

Resiniporus Zmitr., Folia Cryptogamica Petropolitana 6: 98, 2018.

Type species - Polyporus resinascens Romell, Arkiv før Botanik 11 (3): 20, 1911.

Basidiome of ceriporioid habitus, annual, orbicular, confluent into large effused patches or local stalactite-like formations; hymenophore poroid, ceraceous, with resinous deposits. Subiculum white to cream, soft-fibrous. Hyphal system pseudodimitic. Generative hyphae with clamp connections, hyaline, often encrusted by resinous granules, CB-. Pseudoskeletal hyphae thickwalled, yellowish, with scattered phanerochaetoid clamps, CB+. Leptocystidia hyphoid, 
intrahymenial. Basidia utriform, with central constriction, 4-spored, with clamp at the base. Basidiospores oblong-ellipsoid to lacrymoid thin-walled, IKI-, CB-. Causing a white rot.

Remarks - Resiniporus was established for Polyporus resinascens Romell and Poria pseudogilbescens Pilát, two closely related species classified in Ceriporiopsis and separated only based on basidiospore dimensions. The separation is however, supported by molecular data (Tomšovský et al. 2010). We adopt here a broad concept of Ceriporiopsis even it is clear the genus is polyphyletic.

Resinoporia Audet, Mushrooms nomenclatural novelties 7: 1, 2017.

Type species - Physisporus crassus P. Karst., Bidr. Känn. Finl. Nat. Folk 48: 319, 1889.

Basidiome perennial, resupinate, soft when fresh, hard and brittle when dry, separable, odor pleasant, taste bitter, margin white, smooth and narrow; pore surface white to cream, tubes white to slightly yellowish as if partly soaked with resinous substances, especially in old specimens, young parts of tubes distinct, lower parts white, disintegrated and crumbly with a cheesy consistency. Hyphal system dimitic; generative hyphae with clamps, skeletal hyphae thick-walled to solid, sinuous, unbranched to occasionally dichotomously branched, non-amyloid, 3-5 $\mu \mathrm{m}$ in diam; microscopical preparations often filled with oily to resinous, irregular globules. Cystidia none, but fusoid, non-projecting cystidioles usually abundant among the basidia. Basidia clavate, 4sterigmate, with a basal clamp. Basidiospores broadly cylindrical to oblong ellipsoid, hyaline, smooth, negative in Melzer's reagent. Causing a brown rot in dead conifers.

Remarks - According to Audet (2017g), Resinoporia differs from Antrodia s.str. or Amyloporia s.str. by abundant resinous matter occurs in the context and tubes, tortuous and variably inflated skeletal hyphae, basidia broadly clavate to barrel-shaped, with thickened walls after spore detachment. The genus circumscribes the Antrodia crassa group as selected by Spirin et al. (2015), but more studies are needed to elucidate to relationships in the Antrodia crassa group.

Rhizochaete Gresl., Nakasone \& Rajchenb., Mycologia 96(2): 261, 2004.

Type species - Rhizochaete brunnea Gresl., Nakasone \& Rajchenb., Mycologia 96(2): 264, 2004.

Basidiome resupinate, effused, pellicular to membranaceous, subceraceous when fresh, coriaceous when dry, hymenophore smooth to slightly tuberculate, velutinous, yellowish, orange or brownish coloured, turning red to violet with $\mathrm{KOH}$, margin distinct, fimbriate to fibrillose, hyphal cords usually abundant, turning red to violet in $\mathrm{KOH}$. Hyphal system monomitic, generative hyphae with clamps or simple-septa, thin to thick-walled, usually encrusted with dark yellow to yellowish brown granules that dissolve in $\mathrm{KOH}$ turning the solution pale violet; some hyphae also encrusted with hyaline crystals that do not dissolve in $\mathrm{KOH}$. Cystidia as a rule present, cylindrical to subfusiform, thin to thick-walled, encrusted. Basidia clavate to subcylindrical, with 4-sterigmata. Basidiospores cylindrical to ellipsoid, smooth, thin-walled, IKI-.

Remarks - Rhizochate is morphologically and phylogenetically closely related to Phanerochate, but it mainly differs in the characteristic basidiome and hyphal cords reaction to $\mathrm{KOH}$, that turns them red or violet. It also reminds some species of Crustoderma by the tubular cystidia with thickened walls but differs in the crystal and granular encrustations (cf. Greslebin et al. 2004).

Rhizoctonia DC., in Lamarck \& De Candolle, Fl. franç., Edn 3 (Paris) 2: 110, 1805.

Type species - Rhizoctonia solani J.G. Kühn, Ann. Sper. agr., N.S.: 224, 1858.

Remarks - A name based on an anamorph. Anamorphic state of Thanatephorus Donk.

Rhizoporia Audet, Mushrooms nomenclatural novelties 8: 1, 2017.

Type species - Antrodia hyalina Spirin, Miettinen \& Kotir., Mycol. Prog. 12(1): 56, 2013.

Remarks - According to the original description by Audet (2017h), Rhizoporia differs from Antrodia s.str. by basidiomata with margin byssoid, poorly developed rhizomorphs, tubes first 
fleshy, later soft leathery, in some cases partly agglutinated, often easily crumbling, subiculum with generative hyphae somewhat thick-walled, smaller basidia, often slightly constricted in the middle, and, cylindrical, mostly slightly curved but also straight basidiospores. The new genus is supported by the molecular analyses in Ortiz-Santana et al. (2013) and Spirin et al. (2016).

Rhodonia Niemelä, Karstenia 45(2): 79, 2005.

Type species - Polyporus placenta Fr., Öfvers. K. Svensk. Vetensk.-Akad. Förhandl. 18(1): 30, 1861.

Basidiome annual, effused, poroid, fairy thick, juicy and soft, pale rose-colored or white. Hyphal system monomitic, hyphae with clamp connections, at first thin-walled but in mature basidiome thick-walled; cystidia absent; basidia clavate, tetrasterigmate, with a basal clamp; basidiospores hyaline, smooth, IKI-, thin-walled, cylindrical slightly curved. Causing a brown rot on coniferous trees.

Remarks - Rhodonia was the first brown-not polypore in which was completely sequenced the complete genome. The type species of Rhodonia appears nested within Amyloporia sensu lato, but according to Justo et al. (2017), at the moment it is not clear what is the best taxonomic solution for this group (one or multiple genera). Traditionally, Polyporus placenta has been classified in Postia-Oligoporus with other monomitic polypores that causes a brown rot.

Rickiopora Westph., Tomšovský \& Rajchenb., Fungal Biology 120(8): 1005, 2016.

Type species - Daedalea latemarginata Rick, in Rambo (Ed.), Iheringia, Sér. Bot. 7: 263, 1960. (=Antrodiella angulatopora Ryvarden, Mycotaxon 28(2): 525, 1987).

Basidiomes effuse-reflexed to pileate, whitish, soft and somewhat flexible when fresh, becoming brittle and corky to tough when dried. Hymenophore poroid, pores mostly angular, splitted in parts. Hyphal system monomitic to pseudo-dimitic, generative hyphae clamped, thin to thick-walled, thick-walled hyphae with sparse clamps, hyphae metachromatic, IKI-. Cystidia absent, but cystidioles with variable shape abundant in the hymenium. Basidiospores subglobose to ellipsoid, hyaline, smooth, thin-walled, IKI-.

Remarks - Rickiopora is characterized by effused-reflexed basidiomes, often with large resupinate parts, with angular and somewhat split pores and the presence of a monomitic hyphal system with thin to thick-walled metachromatic generative hyphae. Phylogenetically, Rickiopora is included in the residual polyporoid clade (Binder et al. 2013) in the vicinity of Climacocystis borealis (Fr.) Kotl. \& Pouzar (Westphalen et al. 2016).

Rigidoporopsis I. Johans. \& Ryvarden, Trans. Br. mycol. Soc. 72(2): 192, 1979.

Type species - Rigidoporopsis amylospora I. Johans. \& Ryvarden, Trans. Br. mycol. Soc. 72(2): 192, 1979.

Remarks - A synonym of Amylosporus Ryvarden.

Rigidoporus Murrill, Bull. Torrey bot. Club 32(9): 478, 1905.

Type species - Polyporus micromegas Mont., Annls Sci. Nat., Bot., sér. 2 17: 128, 1842.

Basidiome annual to perennial, coriaceous to bony hard when dry, resupinate to pileate, reddish orange to pinkish, isabelline or ochraceous; pileus tomentose to glabrous, usually zonate. Pore surface concolorous, in some species becoming gray to almost black on drying; context dense and fibrous. Hyphal system monomitic to apparently dimitic, generative hyphae with simple septa, variable in width and wall thickness, in some species strongly sclerified skeletoid hyphae. Cystidia absent or present, encrusted at the apex, cystidiols mammillate, smooth. Basidia clavate, 4sterigmate, simple-septate at the base. Basidiospores ovoid to globose, smooth, thin-walled, negative in Melzer's reagent. Causing a white rot in hardwoods, rarely in coniferous wood.

Remarks - Rigidoporus is close to Oxyporus sharing the same type of generative hyphae and cystidia in most species. Basidiome of all species of Oxyporus are usually light colored, and the thick-walled cystidia are hymenial and not tramal as in Rigidoporus. In addition, the mammillate 
cystidiols of Rigidoporus are unknown in Oxyporus. See also Bernicchia \& Gorjón (2020).

Riopa D.A. Reid, Revue Mycol., Paris 33: 244, 1969.

Type species - Riopa davidii D.A. Reid, Rev. Mycol, (Paris) 22: 47, 1969 (=Polyporus metamorphosus Fuckel, Jahrb. Nassauischen Vereins Naturk. 27-28: 87, 1874).

Basidiome white, resupinate with shallow pores, 2-5 per mm. Hyphal system monomitic, clamps absent. Hyphal thin- to slightly thick-walled, similar throughout the basidiome, hyphae not swollen, wider (3-5 $\mu \mathrm{m}$ in diameter) in subiculum, a bit narrower in trama (2.8-3.5 $\mu \mathrm{m})$. Hymenial branching corymb-like. Thin-walled, poorly differentiated hymenial cystidia and conidia in one species. Spores curved cylindrical, allantoid, thin-walled, mid-sized (4.5-6.5 × 2-3 $\mu \mathrm{m})$.

Remarks - Reid (1969) described Riopa as a monotypic genus with Riopa davidii. Miettinen et al. (2016) studied the type of Riopa davidii, and it turned out to be a more recent synonym for Ceriporia metamorphosa (Fuckel) Ryvarden \& Gilb. According to Justo et al. (2017), Riopa belongs to Phanerochaetaceae Jülich with other resupinate polypores and corticioid fungi.

Ripexicium Hjortstam, Mycotaxon 54: 191, 1995.

Type species - Trechispora spinulifera Jülich, Persoonia 8: 437, 1976.

Basidiome resupinate, effuse, closely adnate, firm membranaceous to corneous when dry, hymenial surface distinctly hydnoid. Hyphal system monomitic, hyphae with clamps, some septa with ampullaceous swellings, hyaline to pale yellowish. Cystidia absent. Basidia suburniform to clavate, with 4-sterigmata, and a basal clamp. Basidiospores subglobose, verrucose, thin-walled, inamyloid, indextrinoid, with a weakly cyanophilous reaction.

Remarks - Ripexicium was described to accommodate Trechispora spinulifera. Hjortstam (1995) pointed a relation to Mycoacia (Phlebia) by its strongly hydnoid hymenophore with corneous aculei but differing in the verrucose basidiospores and loose basidial layer, not arranged in a dense palisade as in Mycoacia. Hjortstam (1995) also described the hyphae with no typical ampullate septa even Jülich (1976a) in the original description, both Latin, English and in the linedrawings, described and illustrated ampullate swellings. Larsson (1992) excluded T. spinulifera in the revision of the genus Trechispora. The phylogenetic relationships of T. spinulifera remain still unclear (Larsson 2007).

Rodwaya Syd. \& P. Syd., Hedwigia 40: 2, 1901.

Type species - Merulius infundibuliformis Cooke \& Massee, Grevillea 16(no. 79): 73, 1888.

Remarks - A homonym of Rodwaya F. Mueller, 1890 (Burmanniaceae).

Rogersella Liberta \& A.J. Navas, Can. J. Bot. 56(15): 1777, 1978.

Type species - Rogersella asperula Liberta \& A.J. Navas, Can. J. Bot. 56(15): 177, 1978. (=Corticium griseliniae G. Cunn., Bull. N.Z. Dept. Sci. Industr. Res., Pl. Dis. Div. 145: 71, 1963).

Basidiome effused, thin, subceraceous to chalky or farinose, white or cream coloured to yellowish, under the lens the surface reticulate and nodulose to odontioid, margin thinning. Hyphal system monomitic, hyphae fibulate, the walls not or slightly cyanophilous, crystalline material abundant in the context. Cystidia cylindrical to capitate. Basidia subcylindrical to suburniform, with four sterigmata and a basal clamp. Basidiospores hyaline, globose to ellipsoid, the wall thin or slightly thickened, asperulate, non-amyloid, not or slightly cyanophilous.

Remarks - Rogersella is characterized by sharing typical Hyphodontia-like hyphae, odontioid hymenophore, cylindrical cystidia with a subcapitate apex, and asperulate basidiospores. It is easily distinguished from members of Hyphodontia s.l. because it produces asperulate spores (Langer 1994a). It may be distinguished from other species of Trechispora because it lacks ampulliform hyphae and possesses capitate cystidia. The thin- rather than thick-walled, and smaller basidiospores exclude it from Hypochnicium. Phylogenetically it is closely related to Hyphodontia sambuci and other species in Xylodon. 
Roseograndinia Hjortstam \& Ryvarden, Syn. Fung. (Oslo) 20: 40, 2005.

Type species - Grandinia rosea Henn., Bot. Jb. 38: 108, 1907.

Basidiome resupinate, effused, adnate, distinctly grandinioid or with small aculei, ceraceous, rosy pinkish. Hyphal system monomitic, hyphae with simple-septa, thin-walled, sometimes encrusted. Cystidia absent. Basidia narrowly clavate, with 4-sterigmata, and a simple basal septum. Basidiospores ellipsoid to subglobose, smooth, thin-walled, IKI-, CB-.

Remarks - Roseograndinia was created to accommodate Grandinia rosea a common species distributed in South and Eastern Africa and New Zealand. It is morphologically closely related to Phanerochaete but differs in the grandinioid hymenophore with rosy to pinkish colours. It is also characterized by the absence of cystidial elements, simple-septate hyphae, and smooth basidiospores (Hjortstam \& Ryvarden 2005). Phylogenetic relationships are unclear, but it seems to belong to the Polyporales (Larsson 2007b).

Sarcodontia Schulzer, in Schulzer von Müggenburg et al. Verh. zool.-bot. Ges. Wien 16: 41, 1866.

Type species - Sarcodontia mali Schulzer, in Schulzer, Kanitz \& Knapp, Verh. zool.-bot. Ges. Wien 16(Abh.): 41, 1866 (=Sarcodontia crocea (Schwein.) Kotl., Česká Mykol. 7: 117, 1953).

Basidiome resupinate, effused, ceraceous, hymenophore hydnoid with long aculei. Hyphal system monomitic (pseudodimitic), all hyphae with clamps, subicular hyphae thick-walled. Cystidia absent. Basidia clavate, with 4-sterigmata and a basal clamp. Basidiospores ellipsoid to subglobose, smooth, thick-walled, IKI-, CB-.

Remarks - Sarcodontia is characterized by the long aculeate hymenophore, the thick-walled subicular hyphae, and the smooth thick-walled basidiospores (Schulzer et al. 1866). Phylogenetically it seems to belong to the Meruliaceae (Larsson 2007b).

Sarcoporia P. Karst., Hedwigia 33: 15, 1894.

Type species - Sarcoporia polyspora P. Karst., Hedwigia 33: 15, 1894.

Basidiome annual, resupinate to effused-reflexed or sessile, white to light brown, soft when fresh, fragile when dry. Pores angular; context duplex, with a dense dark gelatinous layer next to the tubes and a white, soft-fibrous layer next to the substrate. Hyphal system monomitic, generative hyphae with clamps. Cystidia none. Basidia clavate, 4-sterigmate, with a basal clamp. Basidiospores cylindrical, smooth, hyaline, slightly thick-walled, dextrinoid in Melzer's reagent. On conifers and hardwoods, causing a brown rot.

Remarks - The genus is closely related to Oligoporus sharing the monomitic hyphal system and a distinct brown rot. The dextrinoid reaction of the thick-walled spores is a diagnostic character. According to Justo et al. (2017), Crustoderma, Pycnoporellus, and Sarcoporia appear in the same clade as Sparassis, but without strong support, and their placement in or out the Sparassidaceae needs to be confirmed in future studies.

Sceptrulum K.H. Larss., Index Fungorum 131: 1, 2014.

Type species - Peniophora inflata Burt, Ann. Mo. bot. Gdn 12: 267, 1926 (=Palifer seychellensis Dämmrich \& Rödel, Z. Mykol. 76(2): 211, 2010).

Basidiome resupinate, effused, thin, adnate to membranous. Hyphal system monomitic, hyphae with clamps. Hymenial cystidia fusoid and more or less apically encrusted; subicular hyphoid projections with a double cup encrustation. Basidia short, cylindrical to clavate or pedunculate, with four sterigmata and a basal clamp. Basidiospores elliptical to subcylindrical or slightly allantoid, thin-walled, smooth, IKI-.

Remarks - Sceptrulum is easily recognized by the subicular cystidial elements, some striking thick-walled hyphal projections with a double small umbrella-like cup encrustation. Phylogenetic relationships with other corticioid genera are unclear. See also Gorjón (2012).

Schizopora Velen., České Houby 4-5: 638, 1922. 
Type species - Polyporus laciniatus Velen., České Houby 4-5: 638, 1922 (=Hydnum paradoxum Schrad., Spicil. fl. Germ. 1: 179, 1794).

Basidiome annual to perennial, resupinate to pileate with narrow, imbricate pilei over a decurrent pore layer, pore surface and context white to cream, pores angular, round to split or as irregular teeth, often of variable length and partly flattened. Hyphal system dimitic; generative hyphae narrow to wide, thin to moderately thick-walled with distinct clamps; skeletal hyphae sparsely represented and mostly as very long cystidial organs. Cystidia none, but subulate or bottleshaped cystidiols, often present in the hymenium, enclosed or partly projecting. Basidia clavate to suburniform, with four sterigmata, and a basal clamp. Basidiospores broadly ellipsoid, hyaline, thin-walled, smooth and non-amyloid.

Remarks - Schizopora is obviously closely related to Xylodon but differing mainly in the poroid hymenophore. Phylogenetically, it is included among other species of Hyphodontia s.l. in the same clade of Odontia quercina Pers., the generic type of Xylodon. Is considered a synonym of Xylodon by most authors. See also Bernicchia \& Gorjón (2020).

Sclerodon P. Karst., Bidr. Känn. Finl. Nat. Folk 48: 348, 360, 1889.

Type species - Hydnum strigosum, Sw., K. Vetensk-Acad. Nya Handl. 31(3): 250, 1810.

Remarks - A synonym of Gloiodon P. Karst.

Scopulodontia Hjortstam, in Hjortstam, Roberts \& Spooner, Kew Bull. 53(4): 820, 1998.

Type species - Scopulodontia loricata Hjortstam \& P. Roberts, in Hjortstam, Roberts \& Spooner, Kew Bull. 53(4): 821, 1998 (=Scopulodontia webbii (Berk.) Nakasone, Sydowia 61: 277, 2009).

Basidiome resupinate, closely adnate, dense, hard, stratose and probably perennial, hymenial surface grandinioid, becoming distinctly hydnoid. Hyphal system monomitic, hyphae closely interwoven in a dense tissue, thin-walled, almost smooth, with clamps. Cystidia abundant, often clustered, thin-walled or with slightly thickened walls, encrusted. Basidia short-clavate, with 4sterigmata and a basal clamp. Basidiospores small, ellipsoid, thin-walled, smooth, IKI-, CB-.

Remarks - Scopulodontia is identifiable because of the stratified, rimose, dense basidiomes with rounded tubercles, encrusted, thick-walled cystidia, and small, ellipsoid basidiospores. See Nakasone (2003, 2009).

Scopuloides (Massee) Hjortstam \& Ryvarden. Mycotaxon 9: 509, 1979.

Basionym - Peniophora subg. Scopuloides Massee, J. Linnean Soc., Botany 25: 154, 1889.

Type species - Peniophora hydnoides Cooke \& Massee, in Cooke, Grevillea 16(79): 77, 1888 (=Peniophora rimosa Cooke, Grevillea 9(51): 94, 1881).

Basidiome resupinate, adnate, effused, ceraceous, hymenophore odontioid. Hyphal system monomitic, hyphae without clamps, thin- to slightly thick-walled. Cystidia numerous, conical, thick-walled, heavily encrusted. Basidia narrowly clavate, with 4-sterigmata and without a basal clamp. Basidiospores cylindrical to ellipsoid, thin-walled, smooth, IKI-, CB-.

Remarks - Scopuloides is characterized by its dense ceraceous hymenium and thick-walled, strongly encrusted cystidia. Scopuloides was put in synonymy with Phanerochaete by Burdsall (1985), but it differs in the thin subiculum with a distinct ceraceous structure more typical in Phlebia. The recent molecular study of Wu et al. (2010) shows that most of the phanerochaetoid fungi are distributed here and there in the phlebioid clade, with a complex phylogenetic relationship. Metulodontia also has a quite firm fruitbody and encrusted cystidia but differs in having small sulfopositive gloeocystidia.

Scotoderma Jülich, Proc. K. Ned. Akad. Wet., Ser. C, Biol. Med. Sci. 77(2): 149, 1974.

Type species - Corticium viride Berk., The botany of the Antarctic Voyage II, Flora NovaeZealandiae 2: 184, 1855. 
Basidiome resupinate, effused, membranaceous, hymenial surface smooth, brown tinged with olive-green, subiculum pale brown, margin tomentose. Hyphal system monomitic, hyphae yellowish brown, thin- to slightly thick-walled, clamped, only in the subhymenial region some secondary septa lacking clamps, not incrusted with granules or crystals, about 2.5-5 $\mu \mathrm{m}$ in diam., not inflated, in some hyphae the cytoplasma turning brown in $\mathrm{KOH}$. Cystidia lacking. Basidia large, broadly cylindrical to subclavate, somewhat stalked, basal part of the basidia occasionally produces a lateral swelling, about $100 \mu \mathrm{m}$ long, 4-spored, yellowish, clamped at the base. Basidiospores large, c. $15 \mu \mathrm{m}$ long, ellipsoid, yellowish o pale brown in water and weak $\mathrm{KOH}$ and darkening to blackish brown, finely roughened, somewhat thick-walled, non-amyloid, noncyanophilous.

Remarks - Scotoderma is characterized by the monomitic hyphal system with clamped hyphae, large basidia, absence of cystidia, and large, somewhat thick-walled, finely ornamented basidiospores, yellowish turning darker after some minutes (Jülich 1974b).

Scotomyces Jülich, Persoonia 10: 139, 1978.

Type species - Corticium fallax G. Cunn., Trans. Roy. Soc. New Zealand 82: 309, 1954. (=Hypochnus subviolaceus Peck, Ann. Rep. N.Y. St. Mus. 47: 25, 1894)

Syn.: Hydrabasidium Park.-Rhodes ex J. Erikss. \& Ryvarden.

Basidiome annual, resupinate, effused, adnate, hypochnoid to subceraceous, hymenophore smooth, margin indistinct, not rhizomorphic. Hyphal system monomitic, hyphae with clamps, thinwalled, hyaline. Cystidia lacking. Basidia obconical to narrowly clavate, thin-walled, with 4 large sterigmata and a basal clamp. Basidiospores repetitive, more or less ellipsoid, smooth, thin-walled, guttulate, IKI-, CB-.

Remarks - Scotomyces is characterized by the greyish to violaceous subceraceous basidiome, the obconical basidia with four large sterigmata, and the repetitive ellipsoid basidiospores. The phylogenetic relationships of Scotomyces remain still unclear (Larsson 2007b).

Scytinostroma Donk, Fungus, Wageningen 26: 19, 1956.

Type species - Corticium portentosum Berk. \& M.A. Curtis, Grevillea 2(13): 3, 1873.

Basidiome resupinate, effused, adnate, coriaceous, hymenophore smooth to tuberculate, usually cream to ochraceous coloured. Hyphal system dimitic; generative hyphae with clamps or simple septa, thin-walled, skeletal hyphae densely branched and sometimes forming dendrohyphae or dichohyphae, narrow and thick-walled, strongly dextrinoid and cyanophilous. Cystidia (gloeocystidia) present in most species, SA+ or SA-, frequently with apical schizopapillae. Basidia tubular to utriform, with 4-sterigmata, basally clamped or simple-septate. Basidiospores subglobose to ellipsoid, smooth, variably amyloid.

Remarks - Scytinostroma is morphologically very close to Vararia, differing in the presence of distinct and numerous dichohyphae in Vararia, which gives a looser consistency to the Basidiome, but frequently it is not difficult to find some intermediate forms, as in Vararia maremmana where dichohyphae are not very differentiated and the basidiome presents a tough texture. Several studies (Larsson \& Larsson 2003, Larsson et al. 2004, Binder et al. 2005, Larsson 2007b) include Scytinostroma close to other russuloid members in the family Peniophoraceae Lotsy. Phylogenetic data seem to indicate that dextrinoid hyphae do not define a monophyletic group.

Scytinostromella Parmasto, Consp. System. Corticiac. (Tartu): 171, 1968.

Type species - Peniophora heterogenea Bourdot \& Galzin, Bull. Soc. mycol. Fr. 28(4): 39, 1913.

Basidiome resupinate, effused, crustaceous or membranaceous to pellicular, hymenophore smooth, whitish to pale yellow, margin usually rhizomorphic. Hyphal system dimitic, generative hyphae with clamps, hyaline to pale yellow, thin-walled, skeletal hyphae originated from thinwalled clamped hyphae, narrow, thick-walled. Cystidia of two kinds: 1) gloeocystidia SA+, and 2) 
pseudocystidia, encrusted (not in all species). Basidia suburniform, with 4-sterigmata and a basal clamp. Basidiospores ovoid, broadly ellipsoid or subglobose, smooth or ornamented, thin- to slightly thick-walled, amyloid, CB-.

Remarks - Scytinostromella morphologically seems to be related to other species of Gloeocystidiellum s.l. with gloeocystidia and amyloid and ornamented basidiospores; with Boidinia shares also the basidial morphology but differs in the dimitic hyphal system. According to molecular studies, the phylogenetic relationships of Scytinostromella are still unclear, but it seems to be a polyphyletic genus (Larsson 2007b). Corticium olivaceoalbum Bourdot \& Galzin was chosen by Jülich (1972) as the generic type of the genus Confertobasidium, but it is a dimitic species with gloeocystidia and amyloid spores, now referred to Scytinostromella and not related with other athelioid fungi.

Sebacinella Hauerslev, Friesia 11(2): 95, 1977.

Type species - Sebacinella nodosa Hauerslev, Friesia 11(2): 95, 1977.

Remarks - A synonym of Oliveonia Donk., according to Roberts (1998).

Sebipora Miettinen, Mycol. Progr. 11(1): 144, 2012.

Type species - Sebipora aquosa Miettinen, Mycol. Progr. 11(1): 144, 2012.

Basidiome resupinate to pileate, annual, pure white when fresh, upper surface hairless, azonate, pore surface turning greyish, sometimes with yellow or brown hues, consistency rubberlike but easy to tear apart when fresh, often watery, hard when dry, pores round to angular, mycelial cords absent. Hyphal system monomitic, $\mathrm{CB}-$, IKI-, $\mathrm{KOH}-$, $\mathrm{CRB}+$, hyphae with clamps, crystals present as sandy encrustation in tube mouths and trama or small thorny crystal clusters attached to tramal hyphae, scanty to abundant; sometimes also large rhomboidal plates and irregular crystal clusters in subiculum and upper trama. Cystidia and cystidiols absent. Basidia clavate, with 4 sterigmata, basally clamped. Basidiospores cylindrical, often slightly curved, thin-walled, smooth, IKI-, CB-. Causing a white rot on buried wood.

Remarks - Sebipora aquosa belongs to the 'Cinereomyces clade' sharing with Gelatoporia, Cinereomyces and Obba the resupinate pale-colored and poroid basidiomes, encrustation in trama or tube mouths, medium-sized spores, a hyaline or shiny oily substance in trama. All species in the clade, except Sebipora aquosa, have hyphal walls swelling in $\mathrm{KOH}$ (Miettinen \& Rajchenberg 2012).

Serpula (Pers.) Gray, Nat. Arr. Brit. Pl. (London) 1: 637, 1821.

Basionym - Merulius sect. Serpula Pers., Syn. meth. fung. 2: 496, 1801.

Type species - Merulius destruens Pers., Syn. meth. fung. (Göttingen) 2: 496, 1801 (=Serpula lacrymans (Wulfen) J. Schröt., Meddn Soc. Fauna Flora fenn. 11: 21, 1885)

Basidiome resupinate to effuse-reflexed, or pileate, membranaceous, soft, easily detachable, hymenophore merulioid to poroid, orange to brownish or with olivaceous tints, margin rhizomorphic. Hyphal system dimitic, hyphae polymorphic, generative hyphae with clamps, skeletoid hyphae present in the context, true skeletal hyphae occur in the rhizomorphs. Cystidia absent, hyphoid cystidiols may be present. Basidia clavate, with 4-sterigmata and a basal clamp. Basidiospores ellipsoid to ovoid, smooth, thick-walled (double layered), brownish, not or weakly dextrinoid, cyanophilous.

Remarks - Serpula differs from Leucogyrophana in the presence of skeletals and in the larger basidiospores (Hallenberg 1985). Serpula, Leucogyrophana, Coniophora, and Hydnomerulius belong to the Boletales, and they are phylogenetically closely related to other gastroid or mushroom-forming fungi (Binder \& Hibbett 2006). Zmitrovich \& Spirin (2002), synonymized Serpula to Leucogyrophana and although they are morphologically closely related, molecular studies seem to indicate that they are phylogenetically separated (Larsson 2007b).

Serpulomyces (Zmitr.) Zmitr., in Zmitrovich \& Spirin, Mikol. Fitopatol. 36(1): 20, 2002. 
Type species - Merulius borealis Romell, Ark. Bot. 11(3): 27, 1911.

Basidiome annual, resupinate, membranaceous to ceraceous when dry, hymenophore merulioid to almost smooth after drying. Hyphal system monomitic, hyphae with clamps, thin- to slightly thick-walled, hyaline. Cystidia absent. Basidia clavate, with four sterigmata, and a basal clamp. Basidiospores cylindrical to fusiform, smooth, thin-walled, IKI-.

Remarks - The generic type of Serpulomyces was traditionally classified in Ceraceomyces. It mainly differs from other species in the genus by the clearly merulioid hymenophore and fusiform basidiospores. Its separation seems phylogenetically supported (Larsson 2007b, Binder et al. 2010).

Sidera Miettinen \& K.H. Larss., Mycol. Progr. 10(2): 136, 2011.

Type species - Physisporus lenis P. Karst., in Rabenhorst, Fungi europ. exsicc.: no. 3527, 1886.

Basidiome resupinate, annual to perennial, hymenophore tubular to hydnoid, pores regular, middle-sized to small, aculei less than $1 \mathrm{~mm}$ long, margin thinning out. Hyphal system monomitic or dimitic, generative hyphae with clamps, thin-walled, acyanophilous or slightly cyanophilous, skeletal hyphae relatively loosely arranged, straight and without branches, acyanophilous. Rosettelike crystals usually abundant in subiculum and sometimes in trama, also irregular rhomboidal plates may be present. Cystidia present as cystidiols (hyphidia, thin-walled cystidia) in hymenium, subulate and long-necked (polypores) to cylindrical and slightly capitate (S. lunata), naked, thinwalled, occasionally surrounded by a halo or vesicle with a very thin wall in the tube mouths. Basidia ellipsoid, cylindrical or clavate, with 4-sterigmata, and a basal clamp. Basidiospores allantoid, curved often strongly, thin-walled, smooth, under $5 \mu \mathrm{m}$ long, $\mathrm{CB}^{-}$, IKI-.

Remarks - Sidera is a recently created genus to accommodate the dimitic polypores Skeletocutis lenis (P. Karst.) Niemelä and S. vulgaris (Fr.) Niemelä \& Y.C. Dai, the monomitic polypore Ceriporiopsis lowei Rajchenb., and the monomitic, hydnoid Athelopsis lunata (Romell ex Bourdot \& Galzin) Parmasto. Sidera thus, includes species with monomitic and dimitic hyphal system, poroid and hydnoid hymenophore, and even seemingly morphologically very different, they form a monophyletic, well-defined group with strong support in phylogenetic analysis. It is characterised by white-rot, whitish resupinate fruiting bodies, crystal rosettes on specialised hyphae, and allantoid spores (Miettinen \& Larsson 2011).

Sistotrema Fr., Syst. mycol. (Lundae) 1: 426, 1821.

Type species - Sistotrema confluens Pers., Neues Mag. Bot. 1: 108, 1794.

Basidiome resupinate or pileate, soft, hymenial surface smooth, grandinioid, odontioid or poroid, pellicular, membranous or ceraceous. Hyphal system monomitic, hyphae usually thinwalled, usually with clamps but few species with simple-septate hyphae, often and characteristically with oily content, frequently ampullate at the septa. Cystidia present or absent. Basidia urniform, mostly with 6-8-sterigmata. Basidiospores varying in shape, smooth, thinwalled, IKI-.

Remarks - Sistotrema is a well delimited genus by the urniform basidia with usually 6-8sterigmata and the hyphae with oily inclusions (Eriksson et al. 1984, Gorjón \& Hallenberg 2008). It contains terricolous to lignicolous species, but recently mycorrhizal associations have also been indicated (Nilsson et al. 2006). Phylogenetically, Sistotrema is polyphyletic belonging to the Hydnaceae in the Cantharellales, closely related to Hydnum, Clavulina, and Membranomyces (Moncalvo et al. 2006).

Sistotremastrum J. Erikss., Symb. bot. upsal. 16(1): 62, 1958.

Type species - Sistotremastrum suecicum Litsch. ex J. Erikss., Symb. bot. upsal. 16(1): 62, 1958.

Basidiome resupinate, effused, thin, hymenophore smooth, porulose to reticulate, whitish. Hyphal system monomitic, hyphae clamped, thin-walled, hyaline. Cystidia absent. Basidia more or 
less clavate to tubular, often constricted, 4-8-sterigmata, with a basal clamp. Basidiospores ellipsoid to cylindrical or suballantoid, smooth, thin-walled, IKI-.

Remarks - Sistotremastrum resembles superficially Sistotrema in the number of sterigmata but differing in the shape of the basidium and in the nature of the hyphae. Molecular studies by Larsson et al. (2004), Binder et al. (2005) and Larsson (2007b) include Sistotremastrum in the trechisporoid clade and not related to Sistotrema as was pointed out by Eriksson et al. (1984).

Sistotremella Hjortstam, in Eriksson, Hjortstam \& Ryvarden, Cortic. N. Eur. (Oslo) 7: 1379, 1984.

Type species - Sistotremella perpusilla Hjortstam, in Eriksson, Hjortstam \& Ryvarden, Cortic. N. Eur. (Oslo) 7: 1381, 1984.

Basidiome resupinate, adnate, effused, hymenophore surface smooth, margin indeterminate. Hyphal system monomitic, hyphae with or without clamps, smooth, thin-walled. Cystidia present or absent. Basidia urniform with 6-8-sterigmata. Basidiospores thick-walled, non-amyloid, cyanophilous.

Remarks - Sistotremella is morphologically close to Sistotrema, differing in the thick-walled basidiospores (Eriksson et al. 1984). Phylogenetically, the systematic position of Sistotremella is unclear (Larsson 2007b).

Skeletocutis Kotl. \& Pouzar, Česká Mykol. 12(2): 103, 1958.

Type species - Polyporus amorphus Fr., Observ. mycol. (Havniae) 1: 125, 1815.

Basidiome annual to perennial, resupinate to pileate, white, cream pink to lilac, often slightly discoloured when dry. Pores usually small, many species with a dense cartilaginous zone above the tube layer. Hyphal system di- to trimitic, generative hyphae with clamps, often encrusted, especially in the dissepiments, skeletal hyphae hyaline. Cystidia absent, cystidioles present in most species. Basidia clavate, 4-sterigmate, with a basal clamp. Basidiospores cylindric to ellipsoid, smooth, hyaline, negative in Melzer's reagent. Causing a white rot.

Remarks - Skeletocutis is morphologically related to Diplomitoporus and Antrodiella. Except for the finely encrusted hyphae of Skeletocutis, these genera share the same basic dimitic hyphal system with skeletal hyphae and clamped generative hyphae, smooth non-amyloid spores and lack of cystidia and the type of white rot. According to Justo et al. (2017), Skeletocutis is classified in Incrustoporiaceae, with Incrustoporia, Piloporia and Tyromyces, a group of polypores producing a white rot with encrusted generative hyphae in the dissepiments, but the generic limits of this genera are still unclear.

Skeletohydnum Jülich, Persoonia 10(3): 331, 1979.

Type species - Epithele nikau G. Cunn., Trans. Roy. Soc. N.Z. 83: 629, 1956.

Basidiome resupinate, effused, adnate, membranaceous. Hymenial surface minutely hydnoid with sterile aculei (hyphal pegs) consisting of hyaline skeletal hyphae. Hyphal system dimitic with skeletal hyphae. Generative hyphae hyaline, cylindrical to torulose, thin-walled, with clamps. Hyaline dendrohyphidia or torulose paraphysoid hyphae present. Cystidia absent. Basidia distinctly constricted in the middle, with a basal clamp, four-spored. Basidiospores hyaline, elongated, ellipsoid, thin-walled, smooth, not amyloid.

Remarks - Skeletohydnum is characterized among the epitheliod fungi by the presence of skeletal hyphae in the core of the hyphal pegs and thin-walled, acyanophilous, and negative in Melzer's reagent basidiospores. Epithelopsis differs in the thick-walled and cyanophilous basidiospores. Sleletohydnum was synonymised to Epithele by Boidin \& Lanquetin (1983) and Hjortstam \& Ryvarden (2005).

Skvortzovia Bononi \& Hjortstam, Mycotaxon 28: 12, 1987.

Type species - Odontia furfurella Bres., Mycologia 17: 71, 1925.

Basidiome resupinate, effused, hymenial surface distinctly odontioid, moderately fragile. Hyphal system monomitic, all hyphae with clamps, hyphae in the trama of the aculei slightly 
conglutinated, thin- to moderately thick-walled. Cystidia (leptocystidia) subulate with a rounded apex, or more or less capitate to lageniform. Basidia clavate, with 4-sterigmata, and a basal clamp. Basidiospores allantoid to subreniform, smooth, thin-walled, IKI-, CB-.

Remarks - Skvortzovia reminds hydnoid Phlebia but is separated by a more fragile basidiome and loose hymenium (Hjortstam \& Bononi 1987). It belongs to the Hymenochaetales in the Rickenella family, and probably it is phylogenetically related to Resinicium.

Sphaerobasidium Oberw., Sydowia 19: 57, 1965.

Type species - Xenasma minutum J. Erikss., Symb. bot. upsal. 16(1): 65, 1958.

Basidiome resupinate, adnate, effused, very thin, hymenophore smooth, margin indeterminate. Hyphal system monomitic, hyphae with clamps, smooth, thin-walled. Cystidia (leptocystidia) present. Basidia subglobose to obconical with a basal clamp, with 4-sterigmata. Basidiospores smooth, thin-walled, IKI-.

Remarks - Sphaerobasidium is mainly characterized by the subglobose basidia and the presence of leptocystidia. The phylogenetic position of Sphaerobasidium is still controversial, according to Binder et al. (2005) it is related to Repetobasidium. This relationship was firstly stated by Eriksson et al. (1984) according to morphological characters; however, in another molecular study by Larsson (2007b), it is placed in the vicinity of Tubulicrinis.

Sphaerophora Bonord., Abh. naturforsch. Ges. Halle 2: 52, 1870.

Type species - Thelephora byssoides Pers., Syn. meth. fung. (Göttingen) 2: 577, 1801.

Remarks - A later homonym of Sphaerophora (A. H. Hassall) J. Lindley 1845 (Bacillariophyceae) and a synonym of Amphinema P. Karst.

Spiniger Stalpers, Proc. K. Ned. Akad. Wet., Ser. C, Biol. Med. Sci. 77(4): 402, 1974.

Type species - Cunninghamella meineckella A.J. Olson, Phytopathology 31: 1076, 1941.

Remarks - Spinger was proposed by Stalpers (1974) to accommodate imperfect states of aphyllophoroid basidiomycetes. Originally was proposed for the anamorphic state of Heterobasidium annosum (Fr.) Bref., so can be considered a synonym of Heterobasidium Massee.

Sporocephalium Chevall., Fl. Gén. Env. Paris (Paris) 1: 59, 1826.

Type species - Haplotrichum capitatum Link, in Willdenow, Sp. pl., Ed. 4 6(1): 52, 1824.

Remarks - A name based on an anamorph. Anamorphic state of Botryobasidium Donk.

Stecchericium D.A. Reid, Kew Bull. 17(2): 270, 1963.

Type species - Steccherinum fistulatum G. Cunn., Trans. Roy. Soc. N.Z. 85(4): 598, 1958.

Basidiome annual, membranous, effused-reflexed to pileate, hymenial surface aculeate. Hyphal system dimitic, generative hyphae with clamps, thick-walled, hyaline, staining, branched, skeletal hyphae thin-walled, hyaline, staining, and forming conducting hyphae arising in the context, traversing aculei and projecting slightly at aculei apices, naked, filled with refractive oily contents. Paraphyses subclavate. Hymenial layer a dense palisade of basidia, paraphyses and conducting hyphae. Basidia subclavate, with 4-sterigmata. Basidiospores oval or obovate, small, usually up to $3 \mu \mathrm{m}$, smooth, hyaline, thin-walled.

Remarks - Stecchericium resembles Steccherinum but differs in the presence of conducting hyphae and absence of encrusted skeletocystidia (Reid 1963).

Steccherinum Gray, Nat. Arr. Brit. Pl. (London) 1: 597, 651, 1821.

Type species - Hydnum ochraceum Pers., in Gmelin, Syst. Nat., Edn 13 2: 1440, 1792.

Synonym - Mycoleptodon Pat.

Basidiome resupinate, effuse-reflexed to subpileate or pileate, membranaceous, hymenophore odontioid to hydnoid, usually cream coloured with pinkish, orange, violaceous or brown tints, margin differentiated, frequently fibrillose. Hyphal system dimitic or pseudodimitic, generative 
hyphae thin-walled, with clamps or simple-septa, skeletal hyphae thick-walled, with some simple septa, some with characteristically encrusted apex penetrating as pseudocystidia into the hymenial layer. Basidia subclavate, with 4-sterigmata and usually with a basal clamp. Basidiospores ellipsoid to subcylindrical, smooth, thin-walled, IKI-, cyanophilous.

Remarks - Steccherinum is characterized by its odontioid hymenophore, encrusted pseudocystidia and small, smooth basidiospores. In a study by Boidin et al. (1998), Meripilus and Steccherinum form a monophyletic group together with Antrodiella, Junghuhnia, Galzinia, Spongipellis, Physisporinus, Hypochnicium, and Rigidoporus in the order Hyphodermatales. Kim \& Jung (2000) included Diplomitoporus, Steccherinum, Meripilus, Columnocystis, Junghuhnia, Albatrellus, Antrodiella, Ceriporiopsis and Spongipellis in the family Steccherinaceae. Larsson (2007b) included Steccherinum in the family Meruliaceae with Junghuhnia as the closest related genus (Junghuhnia differs morphologically only by its poroid hymenophore), these results are in accordance with previous studies by Hibbett \& Binder (2002). Miettinen \& Ryvarden (2016) and Westphalen et al. (2018) and Bernicchia \& Gorjón (2020) consider Junghuhnia as a synonym of Steccherinum.

Sterellum P. Karst., Bidr. Känn. Finl. Nat. Folk 48: 405, 1889.

Type species - Thelephora pini Schleich. ex Fr., Observ. mycol. (Havniae) 1: 154, 1815.

Remarks - A synonym of Peniophora Cooke.

Stereofomes Rick, Egatea 13: 435, 1928.

Type species - Stereofomes nodulosus Rick, Egatea 13: 435, 1928.

Remarks - A synonym of Scytinostroma Donk. See Boidin \& Lanquetin (1997).

Stereogloeocystidium (Rick) Rick, Brotéria, sér. Ci. Nat. 9: 79, 1940.

Type species - Stereogloeocystidium lobatoplicatum Rick, Rick, Ann. K. K. naturh. Hofmus.

Wien 9: 79, 1940. (=Stereum viridans Lloyd., Mycol. Writ. 7: 1339, 1925).

Remarks - A synonym of Podoscypha Pat.

Stereophlebia Zmitr., Folia Cryptogamica Petropolitana 6: 98, 2018.

Type species - Grandinia tuberculata Berk. \& M.A. Curtis, Hooker's J. Bot. Kew Gard. Misc. 1: 237, 1849.

Remarks - A synonym of Phlebia Fr. Stereophlebia is here considered a synonym of Phlebia and waiting for phylogenetic and sexual studies we prefer to adopt a wide concept of Phlebia.

Stereophyllum P. Karst., Hedwigia 28: 190, 1889.

Type species - Stereophyllum pallens P. Karst., Hedwigia 28: 191, 1889.

Remarks - A synonym of Cyphellostereum D.A. Reid.

Stereopsis D.A. Reid, Nova Hedwigia, Beih. 18: 290, 1965.

Type species - Thelephora radicans Berk., J. Bot., London 3: 190, 1844.

Basidiome flabelliform to infundibuliform, stereoid, stipitate, upper sterile surface and hymenophore smooth to rugose. Hyphal system monomitic, hyphae with simple-septa or clamps, thin-walled. Gloeocysitidia usually absent. Basidia clavate, with 4-sterigmata, with a simple septum or a basal clamp. Basidiospores ellipsoid to subglobose, smooth, thin-walled, IKI-, CB-.

Remarks - Stereopsis is closely related to Cotylidia, but it differs in the absence of cystidia. The phylogenetical relationships remain still unclear (Larsson 2007b).

Stereum Hill ex Pers., Neues Mag. Bot. 1: 110, 1794.

Type species - Thelephora hirsuta Willd., Fl. berol. prodr.: 397, 1787.

Synonym - Haematostereum Pouzar. 
Basidiome annual to perennial, resupinate, effused, effuse-reflexed to pileate, upper sterile surface glabrous to tomentose or hirsute, hymenophore smooth to slightly tuberculate, usually with light colours, some species excreting a reddish liquid when cut or touched. Hyphal system dimitic, generative hyphae simple-septate, thin- to thick-walled, skeletal hyphae thick walled. Cystidia of several kinds: skeletocystidia, acutocystidia, acanthocystidia. Basidia clavate to tubular, with 4sterigmata, simple-septate at the base. Basidiospores ellipsoid to cylindrical, smooth, thin-walled, amyloid.

Remarks - Stereum is one of the easily recognized genus at macroscopic level due to its characteristic effuse-reflexed to pileate basidiome with a usually yellowish to orange coloured smooth hymenophore. Some confusion exists regarding to the species because many of them have been described under different names, and too many forms and varieties are found in the literature based only on slight macroscopic differences such as shape, size and/or colour of the basidiome (Jahn 1971). Phylogenetically Stereum is included among other russuloid species; the conducting hyphae, functionally or not, seem to be homologous to the gloeoplerous hyphae present in the rest of the russuloid lineage (Larsson \& Larsson 2003).

Stromatoscypha Donk, Reinwardtia 1: 218, 1951.

Type species - Poria fimbriata Pers., Neues Mag. Bot. 1: 109, 1794.

Remarks - A synonym of Porotheleum Fr.

Subantrodia Audet, Mushrooms nomenclatural novelties 9: 1, 2017.

Type species - Agaricus juniperinus Murrill, Bull. Torrey bot. Club 32(2): 85, 1905.

Remarks - According to Audet (2017i) Subantrodia differs from Antrodia s.str. by smaller basidia, chlamydospores from trama, and by basidiospores with no tapering distal end.

Subulicium Hjortstam \& Ryvarden, Mycotaxon 9: 511, 1979.

Type species - Peniophora lauta H.S. Jacks., Can. J. Res., Section C 26: 129, 1948.

Basidiome resupinate, effused, adnate, hymenophore smooth, pilose by projecting cystidia. Hyphal system monomitic, hyphae with simple-septa, distinct and with thin- to thickened walls. Cystidia of two kinds: 1) subulate, lateral, smooth or encrusted, thick-walled, IKI-, 2) gloeocystidia present or absent, SA-. Basidia clavate or subcylindrical, more or less constricted, with 4sterigmata, with a simple basal septum. Basidiospores globose to subglobose, with thin- to slightly thickened walls, IKI-, not or slightly cyanophilous.

Remarks - Subulicium is a genus with thick-walled, non-amyloid, and lateral cystidia (Hjortstam \& Ryvarden 1979a). The genus is close to Subulicystidium and the main differences are the absence of clamps, globose basidiospores and lateral cystidia. Phylogenetically it could belong to the Hymenochaetales but its relationships are still unclear (Larsson 2007b).

Subulicystidium Parmasto, Consp. System. Corticiac. (Tartu): 120, 1968.

Type species - Hypochnus longisporus Pat., J. Bot. Morot 8(12): 221, 1894.

Basidiome resupinate, effused, more or less arachnoid, smooth to slightly velutinous by the projecting cystidia, margin undifferentiated. Hyphal system monomitic, hyphae clamped, encrusted. Cystidia subulate, characteristically encrusted with rectangular crystals. Basidia urniform, with 4sterigmata and a basal clamp. Basidiospores fusiform to sigmoid, smooth, hyaline, IKI-.

Remarks - Subulicystidium is above all characterized by the particular cystidia encrusted with rectangular crystals; the ornamentation consists of two rows of ribbon-shaped structures, arranged crosswise to the main axis of the cystidia, the free ends of these structures are double-refractive. (Jülich 1975, 1984). Molecular studies by Hibbett \& Binder (2002) and Larsson et al. (2004) show that Subulicystidium is closely related to Tubulicium vermiferum and both are included in the trechisporoid clade next to other Trechispora species, even if they do not share the shame morphological and ecological characters. 
Suillosporium Pouzar, Česká Mykol. 12: 31, 1958.

Type species - Pellicularia cystidiata D.P. Rogers, Farlowia 1: 101, 1943.

Basidiome resupinate, effused, hymenophore flocculose to porulose, whitish, margin undifferentiated. Hyphal system monomitic, hyphae with clamps, thin- to thick-walled. Cystidia (septocystidia) with clamped septa, thin-walled, more or less encrusted. Basidia round, shortly clavate, with 4-sterigmata, and a basal clamp. Basidiospores fusiform to navicular, smooth, thinwalled, IKI-, weakly cyanophilous.

Remarks - Suillosporium by the nature of hyphae, basidia and basidiospores, seems to be related to Botryobasidium, but phylogenetically its relationships remain still unclear (Larsson 2007b).

Syzygospora G.W. Martin, J. Wash. Acad. Sci. 27: 112 (1937)

Type species - Syzygospora alba G.W. Martin, J. Wash. Acad. Sci. 27: 112 (1937)

Remarks - Syzygospora has traditionally been included in the Corticiaceae (e.g. Eriksson \& Ryvarden 1973, under Christiansenia) because of the holobasidia. The gelatinous basidiomes, the mycoparasitic behavior, the presence of tremelloid haustorial branches, the basidiospores germinating by budding and the structure of the septal pores show, however, that they should be placed amongst the heterobasidiomycetes (Diederich 1996).

Szczepkamyces Zmitr., Folia Cryptogamica Petropolitana 6: 52, 2018.

Type species - Trametes campestris Quél., Mém. Soc. Émul. Montbéliard, Sér. 2 5: 286, 1872.

Basidiome annual, persistent, nodulose, cushion-shaped, upper margin forms pseudopilei. Context white to cream, fibrous. Hyphal system dimitic, generative hyphae clamped, skeletal hyphae hyaline, regularly branched, dendrites with inflated axis, appendages sympodially branched. Cystidia absent. Basidia clavate to suburniform, 4-spored, with a basal clamp. Basidiospores large, smooth, fusoid, IKI-, CB-.

Remarks - Trametes campestris Quél. is similar in some respects to species in Polyporus by the binding hyphae Bovista-type. It is very easy to recognize by the pulvinate basidiome, large basidia and basidiospores. It has previously been classified in Dichomitus and Polyporus. According to Zmitrovich (2018) it forms an independent lineage from a molecular perspective.

Taeniospora Marvanová, Trans. Br. Mycol. Soc. 69(1): 146, 1977.

Type species - Taeniospora gracilis Marvanová, Trans. Br. Mycol. Soc. 69(1): 146, 1977.

Remarks - A name based on an anamorph. Anamorphic state of Leptosporomyces galzinii (Bourd.) Jülich. See also Marvanová \& Stalpers (1987).

Terana Adans., Fam. Pl. 2: 5, 1763.

Type species - Byssus caerulea Lam., Fl. franç. (Paris) 1: 103, 1779.

Synonym - Pulcherricium Parmasto.

Basidiome resupinate, effused, hymenophore smooth to slightly tuberculate, bluish. Hyphal system monomitic, hyphae with clamps. Dendrohyphidia present. Cystidia absent. Basidia clavate, often with dendritical prolongations, with 4-sterigmata, and a basal clamp. Basidiospores ellipsoid, smooth, thin-walled, hyaline, IKI-.

Remarks - Terana is characterized above all by the bluish iridescent basidiome. The phylogenetic relationships are unclear (Larsson 2007b).

Thanatephorus Donk, Reinwardtia 3: 376, 1956.

Type species - Hypochnus cucumeris A.B. Frank, Ber. dt. bot. Ges. 1: 62, 1883 (anamorph Rhizoctonia solani J.G. Kühn, Krankh. Kulturgew.: 224, 1858)

Synonyms - Aquathanatephorus C.C. Tu \& Kimbr., Cejpomyces Svrček \& Pouzar, Oncobasidium P.H.B. Talbot \& Keane, Uthatobasidium Donk, Ypsilonidium Donk. 
Basidiome effused, thin, hypochnoid to ceraceous or subgelatinous, hymenophore smooth, whitish to ochraceous. Hyphal system monomitic, hyphae simple-septate, thin to thick-walled. Cystidia absent. Basidia, ellipsoid to oblong or cylindrical, with (1-)2-4(-8) sterigmata. Basidiospores smooth, globose, ellipsoid, citriform or oblong, hyaline to ochraceous, thin-to slightly thick-walled, usually producing secondary spores by replication. Anamorph present.

Remarks - Thanatephorus is characterized by the repetitive spores, the absence of cystidial elements, and by the oblong to clavate basidia usually arranged in a more or less dense palisade (Hjortstam et al. 1988). It with Ceratobasidium seem to form a monophyletic group in the cantharelloid clade, for further comments see Roberts (1999) and Moncalvo et al. (2006).

Thelephora Ehrh. ex Willd., Fl. berol. Prodr.: 396, 1787.

Type species - Thelephora terrestris Ehrh., Pl. Crypt. Linn. Exsicc.: no. 178, 1787.

Remarks - Many Corticiaceae s.l. were firstly described in Thelephora. The generic type is a mycorhizal stipitate species with a smooth to tuberculate hymenophore, coloured, and angularly echinulate basidiospores. It belongs to the Thelephorales, and mainly some resupinate species of the order are considered in this manual.

Theleporus Fr., Kung. Vet. Akad. Handl. 11: 138, 1848.

Type species - Theleporus cretaceus Fr., Kung. Vet. Akad. Handl. 11: 138, 1848.

Basidiome resupinate, adnate, irregularly poroid, light cream to ochraceous, pores angular or interconnected seemingly labyrinthine, hymenium as a whiter and denser layer restricted to the base of the pores. Hyphal system di-trimitic, generative hyphae with clamps, vegetative hyphae of two types, partly as skeletal hyphae more or less unbranched and parallel in the tube walls, and binding arboriform hyphae present, all vegetative hyphae hyaline to very pale yellowish, not reacting in Melzer's reagent. Dendrohyphidia present or absent. Basidia clavate, with 4-stergimate, and a basal clamp. Basidiospores broadly ellipsoid, thin-walled, smooth, non-amyloid.

Remarks - Theleporus is related to Grammothele and the main difference is the di-trimitic hyphal system, with straight and arboriform hyphae, and vegetative hyphae not reacting in Melzer's reagent. In Grammothele skeletal hyphae are dextrinoid and darkening with age, this make the basidiomes of Grammothele darker with age. Contrary, Theleporus keep their whitish to pale colours (Ryvarden 1991).

Thujacorticium Ginns, Mycologia 80: 69, 1988.

Type species - Thujacorticium mirabile Ginns, Mycologia 80(1): 69, 1988 (=Corticium zurhausenii Bres., Öst. bot. Z. 48: 136, 1898).

Basidiome resupinate, pulvinate, crustaceous, hymenophore smooth, margin more or less abrupt. Hyphal system monomitic, hyphae with clamps, thin- to thick-walled, hyaline. Cystidia absent, cystidiols rare, cylindrical to narrowly clavate or subulate. Basidia clavate, pedunculate, with 4-sterigmata, and a basal clamp. Basidiospores ellipsoid to subcylindrical, smooth, thinwalled, hyaline, IKI-, CB-.

Remarks - Thujacorticium seems to be related to Cerocorticium, but hyphoid elements and larger basidiospores are present in the latter. It differs from Hyphoderma in the typically pedicellate basidium. In addition, the conspicuous development of large crystal when section is mounted in heated cotton blue is an interesting feature (Ginns 1988). Phylogenetically its position is still unclear (Larsson 2007b).

Thwaitesiella Massee, Grevillea 21: 2, 1893.

Type species - Radulum mirabile Berk. \& Broome., J. Linn. Soc., Bot. 14(2): 61, 1875.

Remarks - A synonym of Lopharia Kalchbr. \& MacOwan.

Tinctoporellus Ryvarden, Trans. Br. mycol. Soc. 73(1): 18, 1979. 
Type species - Polyporus epimiltinus Berk. \& Broome, J. Linn. Soc., Bot. 14(no. 73): 54, 1873.

Basidiome resupinate, pore surface bluish to light violet, pores angular. Hyphal system dimitic, generative hyphae with clamps, skeletal hyphae thick-walled, hyaline to light golden yellow in $\mathrm{KOH}$, weakly dextrinoid in Melzer's reagent. Cystidia none. Basidia clavate, with 4sterigmata and a basal clamp. Basidiospores ellipsoid to subglobose, smooth, hyaline and nonamyloid. Causing a white rot and turns the substratum reddish in zones.

Remarks - Tinctoporellus is characterized by the dimitic hyphal system, smooth basidiospores negative in Melzer's reagent, and by turning the substrate reddish. This change of colour to red in the rotten substrate is also present in Porogramme albocincta (Cooke \& Massee) J. Lowe (Ryvarden 1991).

Tofispora G. Langer, Biblthca Mycol. 158: 32, 1994.

Type species - Tofispora repetospora G. Langer \& Ryvarden, in Langer, Biblthca Mycol. 158: 338, 1994.

Basidiome resupinate, loosely adnate, hypochnoid, hymenophore smooth, whitish to ochraceous or, especially when dried or old, yellowish brown to brownish. Hyphal system monomitic, basal hyphae almost hyaline to pale brown, thick-walled, subhymenial hyphae hyaline to subhyaline, slightly narrower and generally thin-walled. All hyphae without clamp connections. Cystidia absent. Basidia stout, with (1)(2)4(5) sterigmata. Basidiospores globose or distinctly biapiculate, thick-walled, with small verrucae, hyaline or brownish, with a distinct apiculus. All species in the genus except Tofispora hebelomatospora producing secondary spores.

Remarks - According to Roberts (1999) a synonym of Thanatephorus Donk but differing in the asperulate basidiospores. At least, the generic type seems to be separated from Thanatephorus by the ornamented basidiospores.

Tomentella Pers. ex Pat., Hyménomyc. Eur. (Paris): 154, 1887. (nom. cons.)

Type species - Corticium ferrugineum Pers., Observ. mycol. (Lipsiae) 2: 18, 1800.

Basidiome annual, resupinate, effused, byssoid, arachnoid, mucedinoid or pelliculose, adherent to separable, sometimes encrusting, fertile areas continuous or discontinuous, red, ferruginous, yellow, tan, brown, green, olive, grey, or black, margin arachnoid, byssoid, villose, fibrillose, or fimbriate, normally paler than the fertile area, hymenial surface smooth, granulose, colliculose, or aculeate, basidiomes often darkening or changing colour in $\mathrm{KOH}$. Hyphal system monomitic or dimitic, generative hyphae often of more than one kind, thin- or thick-walled, septate, clamp connections abundant, normally pigmented; cordons present or absent. Basidia clavate, often with transverse septa, 4-sterigmate. Cystidia absent or present, acuminate, clavate, obclavate or capitulate. Basidiospores normally pigmented some shade of brown, green or red $\left(\right.$ in $\left.\mathrm{H}_{2} \mathrm{O}\right)$ and usually appearing some shade of brown in $\mathrm{KOH}$, variable in shape but usually subglobose to lobed in outline, ornamented.

Remarks - Tomentella is a large genus with numerous and difficult species. For more information see Larsen (1974) and Kõljalg (1996). Species of Tomentella are ectomycorrhizal fungus, being of ecological importance in the forest dynamic.

Tomentellago Hjortstam \& Ryvarden, Mycotaxon 31(1): 40, 1988.

Type species - Tomentellago aeruginascens Hjortstam \& Ryvarden, Mycotaxon 31(1): 40, 1988.

Remarks - A synonym of Amaurodon J. Schröt.

Tomentellastrum Svrcek, Ceska Mykol. 12: 68, 1958.

Type species - Thelephora fuscocinerea Pers., Mycol. eur. (Erlanga) 1: 114, 1822.

Remarks - A synonym of Tomentella Pat. 
Tomentellina Höhn. \& Litsch., Sber. Akad. Wiss. Wien, Math.-naturw. Kl., Abt. 1 115: 1604, 1906. Type species - Tomentellina ferruginosa Höhn. \& Litsch., Beitr. 1: 1604, 1906.

Remarks - A synonym of Tomentella Pat.

Tomentellopsis Hjortstam, Svensk bot. Tidskr. 64(4): 425, 1970.

Type species - Corticium echinosporum Ellis, Bull. Torrey bot. Club 8: 64, 1881.

Basidiome resupinate, separable or rarely adherent to the substratum, arachnoid or pelliculose, continuous, hymenophore smooth, concolor with or turning paler than subiculum, sterile margin usually indeterminate. Hyphal cords absent. Hyphal system monomitic, subicular hyphae simple-septate, cross-shaped branching common, thin-walled, without encrustation, hyaline to pale brown in $\mathrm{KOH}$, subhymenial hyphae simple-septate, thin-walled, hyaline in $\mathrm{KOH}$. Cystidia absent. Basidia up to $40 \mu \mathrm{m}$ long, simple-septate at base, utriform, not stalked, sometimes sinuous, without transverse septa, hyaline in $\mathrm{KOH}$ and in distilled water, 4 sterigmata. Basidiospores up to $7.5 \mu \mathrm{m}$ long in lateral and frontal face, slightly globose or ellipsoid frontal and lateral faces, echinulate, hyaline or rarely very pale brown in $\mathrm{KOH}$ and in distilled water. Chlamydospores absent.

Remarks - Tomentellopsis is defined by the simple-septate hyphae and echinulate basidiospores that are usually hyaline in KOH. See Kõljalg (1996) for more information.

Trechinothus E.C. Martini \& Trichies, Mycotaxon 90(2): 262, 2004.

Type species - Tomentella smardae Pilát, Stud. Bot. Čechoslav. 5: 75, 1942.

Basidiome pellicular, separable, membranaceous, hymenophore smooth, porulose, whitish to pale yellowish, margin indetermiante to fibrillose, rhizomorphs present. Hyphal system monomitic, hyphae with evident clamps, some ampullate septa present, short-celled in the subhymenium, longcelled in the subiculum, becoming brown next to the substrata, hyphal strands originated from subicular hyphae. Cystidia absent. Basidia subcylindrical to clavate, with (2)4-sterigmata, and with a basal clamp. Basidiospores ovoid to dacryoid or ellipsoid, echinulate, thick-walled, hyaline, IKI-, CB-. Chlamydospores numerous in subiculum and in the rhizomorphs, globulose, berry-like, at first hyaline, then typically yellowish to brown.

Remarks - Trechinothus was described to include Tomentella smardae, a deviating species in Tomentella. It is characterized by the echinulate basidiospores with ornamentation that mostly develops during the following stages of maturation, the ampullate hyphae, the presence of numerous chlamydospores in the subiculum and in the rhizomorphs, and the absence of any cyanophilous reaction. For further comments see Martini \& Trichies (2004). Phylogenetically, the systematic position of Trechinothus is unclear (Larsson 2007b).

Trechispora P. Karst., Hedwigia 29: 147, 1890.

Type species - Trechispora onusta P. Karst., Hedwigia 29: 147, 1890.

Synonyms - Cristelloporia I. Johans. \& Ryvarden, Dextrinodontia Hjortstam \& Ryvarden, Echinotrema Park.-Rhodes, Fibriciellum J. Erikss. \& Ryvarden.

Basidiome resupinate or pileate, thin to thick, hymenophore smooth, grandinioid, odontioid or poroid, usually light coloured. Hyphal system monomitic or dimitic, hyphae clamped, usually with typical ampullate septa, subhymenial cells short, skeletal hyphae present in some species. Cystidia usually absent, but some hyphoid cystidia can be present. Basidia short, cylindrical to clavate, with 4-sterigmata and a basal clamp. Basidiospores small, usually subglobose to ellipsoid, rarely allantoid, smooth or ornamented, usually with some thickened walls, IKI-, acyanophilous or with a slight cyanophilous reaction.

Remarks - Trechispora is above all characterized by the fragile basidiome, with a variable hymenophore configuration, and by the frequently ampullate hyphae. According to molecular data, Trechispora belongs to the Hydnodontaceae Jülich with Brevicellicium, Fibrodontia and other genera with no common morphological features as Luellia, Subulicystidium, and Tubulicium (Larsson 2007b). 
Tretomyces K.H. Larss, Kotir. \& Saaren., Ann. Bot. Fennici 48: 41, 2011.

Type species - Byssocorticium lutescens J. Erikss. \& Ryvarden, Cort. North Europe 2: 185, 1973.

Basidiome byssoid, yellowish or greyish, hymenophore smooth. Hyphal system monomitic, hyphae usually with clamps but partly simple-septate. Cystidia absent, but hyphidia may be present. Basidia clavate or subcylindrical, with a basal clamp. Basidiospores globose or subglobose, smooth, with thickened walls, inamyloid, cyanophilous.

Remarks - Tretomyces is characterized by a yellowish, soft, byssoid basidiome, monomitic hyphal system, hyphae with both clamped and simple septa, stalked, relatively small basidia and small, almost spherical, slightly thick-walled cyanophilous basidiospores. It lacks typical bluishgreen colour of Byssocorticium where the generic type was previously classified. Microscopically it is reminiscent of Piloderma, whose hyphae are always simple-septate. It seems that Piloderma is phylogenetically the closest relative (Kotiranta et al. 2011).

Trichaptum Murrill, Bull. Torrey Bot. Club 31: 608, 1904.

Type species - Polyporus trichomallus Berk. \& Mont.

Basidiome annual, resupinate to reflexed, often pileate with imbricate growth, hymenial surface poroid, irpicoid to lamellate, purplish to brownish when old or dry but with a typical violet shadow when young. Hyphal system di-trimitic, generative hyphae with clamps, skeletal hyphae thick-walled, sometimes more or less branched. Cystidia usually as hymenial cystidia encrusted in the apical part, thin- to usually thick-walled. Basidia clavate, with 4-sterigmate, and a basal clamp. Basidiospores cylindrical to allantoid, smooth, thin-walled, hyaline, IKI-. On deciduous or coniferous wood, causing a white rot.

Remarks - Trichaptum is characterized above all by the purplish to the violet hymenial surface, and microscopically by the di- to trimitic hyphal system, encrusted cystidia, and smooth basidiospores without any reaction in Melzer's reagent (Ryvarden 1991).

Trimitiella Dhingra, Mycotaxon 97: 125, 2006.

Validated by Trimitiella Dhingra, Mycotaxon 105: 422, 2008.

Type species - Trimitiella indica Dhingra, Mycotaxon 97: 126, 2006.

Basidiome resupinate, adnate, effused, membranous-ceraceous, hymenial surface smooth, margin turned when drying. Hyphal system trimitic, generative hyphae with clamps, skeletal hyphae thick-walled, acyanophilous, binding hyphae richly branched, thick-walled, context composed of densely interwoven generative, skeletal, and binding hyphae. Dendrohyphidia present. Cystidia absent. Basidia clavate, 4-sterimate, with a basal clamp. Basidiospores broadly ellipsoid, smooth, thin-walled, non-amyloid, CB-, with numerous oil drops.

Remarks - Trimitiella is characterized by the trimitic hyphal system, presence of dendrohyphidia, and smooth non-amyloid basidiospores. Laurilia also possesses a trimitic hyphal system but differs in the encrusted cystidia and amyloid and ornamented basidiospores (Dhingra 2006, Dhingra \& Singh Avneet 2008).

Trulla Miettinen \& Ryvarden, in Miettinen \& Ryvarden, Ann. bot. fenn. 53(3-4): 168, 2016. Type species - Antrodiella dentipora Ryvarden \& Iturr., Mycologia 95 (6): 1066, 2004. Remarks - A homonym of Trulla T.M. Harris 1979 (Pinopsida).

Trullella Zmitr., Folia Cryptogamica Petropolitana 6: 104, 2018.

Type species - Antrodiella dentipora Ryvarden \& Iturr., Mycologia 95 (6): 1066, 2004.

Basidiome of polyporoid, trametoid or fibroporioid habitus, annual, with poroid or odontioid hymenophore. Hyphal system monomitic in sterile context and dimitic in tube trama. Generative hyphae with clamp connections, hyaline, $\mathrm{CB}+$, skeletal hyphae mostly hyaline, fibroid, $\mathrm{CB}^{+}$. Leptocystidia short-clavate. Basidia short clavate, with a central constriction, 4-spored, with clamp 
at the base. Basidiospores phaseoleform to allantoid, smooth, thin-walled, IKI-, CB-. Causing a white rot.

Remarks - The genus Antrodiella Ryvarden \& I. Johans. is similar but differs by dimitic context, acyanophilous generative hyphae and less curved basidiospores (Zmitrovich 2018).

Tsugacorticium Nakasone \& Burds., North American Fungi 7(1): 3, 2012.

Type species - Tsugacorticium kenaicum Nakasone \& Burds., North American Fungi 7(1): 6, 2012.

Basidiome effuse, adnate, soft, smooth, with distinct, abrupt margin. Hyphal system monomitic with clamped generative hyphae. Subhymenium thickening, composed of hyphae and dendrohyphidia. Dendrohyphidia filamentous to frequently branched, smooth. Cystidia subfusiform, thin-walled, hyaline, smooth. Basidia suburniform, elongate, with a distinct stalk, 4sterigmate. Basidiospores globose to subglobose with thin walls, hyaline, smooth, IKI-, CB-.

Remarks - The most striking features of Tsugacorticium are the soft textured basidioma, elongate, stalked suburniform basidia bearing subglobose to globose basidiospores, and large, smooth, branched dendrohyphidia. Also, the thickened subhymenium that constitutes most of the context and is composed primarily of collapsed, deteriorated hymenial elements (Nakasone \& Burdsall 2012).

Tubulicium Oberw., Sydowia 19: 53, 1965.

Type species - Peniophora vermifera Bourdot, Rev. Sci. Bourb. Centr. Fr. 23(1): 13, 1910.

Basidiome resupinate, effused, adnate, hymenial surface smooth, velutinous under the lens. Hyphal system monomitic, hyphae with clamps, thin- to slightly thick-walled. Cystidia (lyocystidia) numerous, conical, multi-rooted, encrusted and covered with dendroid branching hyphae. Basidia clavate, more or less pedunculate, smooth, thin-walled, with 4-sterigmata and a basal clamp. Basidiospores navicular, sigmoid to vermicular, smooth, thin-walled, IKI-, CB-.

Remarks - Tubulicium is characterized by the multi-rooted lyocystidia covered by dendroid hyphae and by the sigmoid smooth basidiospores. According to molecular data, Tubulicium is included in the trechisporoid clade, but there are no common morphological characters to support this phylogenetic relationship (Larsson et al. 2004, Larsson 2007b).

Tubulicrinis Donk, Fungus, Wageningen 26: 13, 1956.

Type species - Thelephora calcea var. glebulosa Fr., Elench. fung. (Greifswald) 1: 215, 1828.

Basidiome resupinate, firmly adnate, often inconspicuous, more rarely thick and cracking in irregular pieces, hymenophore smooth pruinose to porulose, but commonly with a pilose appearance due to the protruding cystidia, whitish, cream or with greyish tint. Hyphal system monomitic, hyphae with clamps. Cystidia (lyocystidia) conspicuous, projecting, cylindrical or conical, with capitate or subulate apex, usually bi-rooted, dissolving in $\mathrm{KOH}$ (5-10\%), mostly encrusted, with a weak or strongly amyloid reaction, capillary lumen ending gradually or more abruptly. Basidia small, thin-walled or sometimes thickened in the basal part, arranged in a dense palisade, often strongly amyloid, with 4-sterigmata and a basal clamp. Basidiospores cylindrical to allantoid or globose to ellipsoid, smooth, thin-walled often, IKI-, CB-.

Remarks - Tubulicrinis was described by Donk to accommodate Corticium glebulosum Bres. (Donk 1956). It comprises a group of well-defined corticioid species characterized by its typical thick-walled (lyocystidia), usually bi-rooted and more or less amyloid cystidia that dissolve totally or partially in $5-10 \% \mathrm{KOH}$. The particular kind of cystidia, variable in shape and size, and the basidiospores, both constitute the most reliable features to distinguish the different species (Oberwinkler 1966). Tubulicrinis shares morphological affinities with other genera such as Dextrinocystis, Tubulicium and Litschaurella. Dextrinocystis is characterized by its dextrinoid lyocystidia and Litschaurella and Tubulicium mainly differ from Tubulicrinis in the multi-rooted lyocystidia with a weak amyloid reaction. For a synopsis of this genera see Hjortstam (2001). According to several DNA molecular analysis, Tubulicrinis belong to the hymenochaetoid clade 
and is related to species of Hyphodontia, Schizopora, Basidioradulum, Resinicium, Sphaerobasidium and Hymenochaete (Hibbett \& Binder 2002, Larsson et al. 2004, Larsson et al. 2006, Larsson 2007b). The last tentative classification of corticioid fungi by Larsson (2007b) recognizes Tubulicrinis in the family Tubulicrinaceae, in the order Hymenochaetales Oberw., with Hyphodontia as the closest related genus. Tubulicium is assigned to the trechisporioid clade (family Hydnodontaceae Jülich, order Trechisporales K.H. Larss.), on the basis of molecular analysis even if Tubulicium is not morphologically related to Trechispora. Tubulicrinis and Tubulicium, belonging to different clades, could indicate that lyocystidia must have evolved at least twice (Larsson et al. 2004).

Tubulicrinopsis Hjortstam \& Kotir., in Kotiranta, Hjortstam, Miettinen \& Kulju, Ann. bot. fenn. 44(2): 129, 2007.

Type species - Tubulicrinopsis ellipsospora Kotir., Hjortstam \& M. Kulju, in Kotiranta, Hjortstam, Miettinen \& Kulju, Ann. bot. fenn. 44(2): 129, 2007.

Basidiome resupinate, effused, hymenophore farinaceous, porulose to grandinioid. Hyphal system monomitic, hyphae with clamps, thick-walled. Cystidia usually absent or present, thickwalled. Basidia clavate to subcylindrical, with 4-sterigmata, with a basal clamp, distinctly thickwalled. Basidiospores ellipsoid to cylindrical, smooth, thin-walled, IKI-.

Remarks - Tubulicrinopsis was described with Tubulicrinopsis ellipsospora as the generic type species and including two more species. In addition, Amauromyces farinaceus Boidin, Lanq. \& Gilles was transferred to Tubulicrinopsis (Kotiranta et al. 2007). Amauromyces pallidus Jülich, the generic type of Amauromyces seems not to be related to A. farinaceus. Tubulicrinopsis is characterized by the thick-walled hyphae and basidia. Tubulicrinis and Sistotremastrum have also somewhat thick-walled basidial bases but differing in the presence of amyloid lyocystidia and basidia with 6-sterigmata respectively.

Tubulixenasma Parmasto, Izv. Akad. Nauk Estonsk. SSR, Ser. Biol. 14: 231, 1965.

Type species - Peniophora vermifera Bourdot, Rev. Sci. Bourb. Centr. Fr. 23(1): 13, 1910.

Remarks - A synonym of Tubulicium Oberw.

Tulasnella J. Schröt., in Cohn, Krypt.-Fl. Schlesien (Breslau) 3.1(25-32): 397, 1888.

Type species - Tulasnella lilacina J. Schröt., in Cohn, Krypt.-Fl. Schlesien (Breslau) 3-1(4): 397, 1888.

Basidiome effused, ceraceous to subgelatinous, typically violaceous grey, but often macroscopically subinvisible, hymenophore smooth. Hyphal system monomitic, hyphae with or without clamp connexions. Hyphidia and/or cystidia present in some species. Basidia clavate to sphaeropedunculate, often pleural and with papillate or snout-like projections, with distinct globose to ellipsoid sterigmata becoming obclavate and then variously extending, separated from the basidium by a simple septum. Basidiospores variable in size and shape, variously globose to vermiform or helicoid, smooth, producing secondary spores by replication, IKI-. Microconidia may also be produced following basidiospore germination. Chlamydospores present in some species.

Remarks - Tulasnella is a cosmopolitan genus of saprobes species, some of them also orchid endomycorrhizal. It is quite easy to recognize by the particular basidia with large obclavate sterigmata that are separated between them and from the rest of the basidia by a simple septum.

Tylospora Donk, Taxon 9: 220, 1960.

Type species - Hypochnus asterophorus Bonord., Handb. Allgem. mykol. (Stuttgart): 160, 1851.

Basidiome resupinate, effused, loosely adnate, hymenophore smooth, arachnoid to byssoid, white to greyish, margin indeterminate. Hyphal system monomitic, all hyphae with clamps, thin- to thick-walled, usually encrusted. Cystidia absent. Basidia clavate, with 4-sterigmata and a basal clamp. Basidiospores lobed or triangular, smooth or ornamented, IKI-. 
Remarks - Tylospora is easily to identify due to the lobed or triangular basidiospores. Tylospora shares many characteristics in hymenial configuration with other athelioid fungi and was included in the Atheliae by Jülich (1972); molecular data support this relationship (Larsson 2007b).

Tylosperma Donk, Fungus 27: 28, 1957.

Type species - Hypochnus asterophorus Bonord., Handb. Allgem. mykol. (Stuttgart): 160, 1851.

Remarks - A later homonym of Tylosperma Botsch. 1952 (Rosaceae).

Tyromyces P. Karst., Revue mycol., Toulouse 3(no. 9): 17, 1881.

Type species - Polyporus chioneus Fr., Observ. mycol. (Havniae) 1: 125, 1815.

Basidiome annual, resupinate to pileate, watery to waxy when young, rigid and fragile when dry; usually with a bitter taste; upper surface white, darkening when drying; hymenophore tubular with round to angular pores, white to cream or greenish; hyphal system monomitic to dimitic, generative hyphae with clamps, skeletal hyphae when present straight, thick-walled, gloeoplerous hyphae present in some species; cystidia absent, but cystidiols sometimes present; basidia clavate, tetrasterigmate, with a basal clamp; basidiospores cylindrical to ellipsoid or ovoid, hyaline, smooth, thin-walled, IKI-. Causing a white rot on hardwoods or conifers. Cosmopolitan genus.

Remarks - From morphology, Tyromyces seems related to Ceriporiopsis, sharing the monomitic hyphal system, and white type or rot, differing mainly in the usually pileate basidiomes in Tyromyces contrary to the resupinate basidiomes of Ceriporiopsis. Phylogenetic relationships of both genera are unclear also taking in consideration of the polyphyletic status of Ceriporiopsis. Tyromyces is nested in Incrustoporiaceae Jülich, but the generic limits of Incrustoporia, Skeletocutis and Tyromyces are not currently settled, and additional sampling of this clade is needed before a taxonomic arrangement can be put forward (Justo et al. 2017).

Uncobasidium Hjortstam \& Ryvarden, Mycotaxon 7: 407, 1978.

Type species - Uncobasidium luteolum Hjortstam \& Ryvarden, Mycotaxon 7(2): 408, 1978.

Basidiome resupinate, effused, membranaceous to byssoid, hymenophore smooth. Hyphal system monomitic, hyphae with clamps, thin- to thick-walled, straight in the subiculum and tortuous in the subhymenium. Cystidia absent. Basidia suburniform, pleurobasidiate, constricted, with a lateral hook-like protuberance, with 4-sterigmata, and a basal clamp. Basidiospores ellipsoid to ovoid, smooth, thin-walled, IKI-, CB-.

Remarks - Uncobasidium is characterized above all by the typical pedunculate basidia with a basal protuberance (Hjortstam \& Ryvarden 1978, Gorjón et al. 2012). Phylogenetic relationships of Uncobasidium are still unclear (Larsson 2007b).

Urnobasidium Parmasto, Consp. System. Corticiac. (Tartu): 38, 1968.

Type species - Gloeocystidium sernanderi Litsch., Svensk bot. Tidskr. 25(3): 437, 1931.

Remarks - A synonym of Sistotrema Fr.

Uthatobasidium Donk, Reinwardtia 3: 376, 1956

Type species - Hypochnus fusisporus J. Schröt., Kryptogamenflora der Schweiz 3(1): 416, 1888.

Remarks - A synonym of Thanatephorus Donk.

Vanderbylia D.A. Reid, J. Soc. Afr. Bot. 39(2): 166, 1973.

Type species - Polyporus vicinus Lloyd, Mycol. Writ. 7(Letter 74): 1331, 1924.

Basidiome annual to perennial, pileate, hard corky to woody hard, pilei applanate, slightly convex, semicircular; pileal surface slightly to distinctly warted, glabrous. Pore surface white to grayish cream; pores round; dissepiments thick, entire. Hyphal system dimitic, generative hyphae hyaline, thin-walled, rarely branched, bearing clamp connections, skeletal hyphae dominating in the 
context, hyaline, subsolid to thick-walled with a wide lumen, branched, interwoven, variably dextrinoid, cyanophilous; hyphae occasionally swollen in KOH. Dichohyphidia-like (coral-like dendrohyphidia) element present at the dissepiment. Cystidia absent. Cystidioles variably present. Basidia clavate, with four sterigmata and a basal clamp connection; basidioles often dominant, in shape similar to basidia, but slightly smaller. Basidiospores subglobose to amygdaliform, nontruncate, hyaline, thick-walled, smooth, strongly dextrinoid, $\mathrm{CB}+$.

Remarks - Vanderbylia is similar to Perenniporia sensu stricto on the basis of the thickwalled basidiospores, but it differs by its pileate, hard corky basidiocarps and strongly dextrinoid skeletal hyphae and subglobose to amygdaliform, non-truncate basidiospores. Molecular studies showed that this genus is distant from Perenniporia sensu stricto (Zhao et al. 2013a).

Vararia P. Karst., Kritisk Öfversigt af Finlands Basidsvampar, Tillägg 3: 32, 1898.

Type species - Radulum investiens Schwein., Trans. Am. phil. Soc., Ser. 2 4(2): 165, 1832.

Synonym - Asterostromella Höhn. \& Litsch.

Basidiome resupinate, effused, adnate, membranaceous, hymenophore smooth, whitish to cream or ochraceous. Hyphal system dimitic, generative hyphae with or without clamps, with differentiate dextrinoid dichohyphae. Cystidia (gloeocystidia) present but variable in number, usually SA+. Basidia utriform, with 4-sterigmata. Basidiospores ellipsoid to subglobose, allantoid to fusoid, smooth (ornamented in some tropical species), amyloid or inamyloid, indextrinoid.

Remarks - Vararia is closely related to Dichostereum and mainly separated by the presence of ornamented basidiospores in the latter. It is also similar in some respects to Scytinostroma, and its generic separation is sometimes a matter of opinion because there are several intermediate stages between the two genera. Phylogenetic analyses show that dextrinoid hyphidia do not define a monophyletic group (Larsson 2007b). Actually, Vararia, Dichostereum, Scytinostroma and Asterostroma, traditionally included in the Lachnocladiaceae on the common presence of the dextrinoid skeletoid elements, are phylogenetically placed in Peniophoraceae belonging to the Russulales (Larsson \& Larsson 2004). The analysis of the little-known tropical species, as in most of the corticioid fungi, will surely clarify the phylogenetic relationships.

Veluticeps (Cooke) Pat., Bull. Soc. mycol. Fr. 10: 78, 1894.

Basionym - Hymenochaete subgen. Veluticeps Cooke, Grevillea 8(48): 148, 1880.

Synonym - Columnocystis Pouzar.

Type species - Veluticeps berkeleyi Cooke, Bull. Soc. mycol. Fr. 10: 78, 1894.

Basidiome resupinate to effuse-reflexed or semipileate, hymenophore smooth or aculeate with hyphal pegs, usually brownish. Hyphal system dimitic, hyphae thin- or thick-walled, hyaline to pale yellowish brown, with or without clamps. Cystidia (skeletocystidia or pseudocystidia) abundant, either single or more commonly in fascicles, thick-walled, apically obtuse, and usually encrusted, pale to dark reddish-brown. Basidia narrowly clavate, with 4-sterigmata and a basal clamp. Basidiospores ellipsoid to cylindrical, often somewhat fusiform, smooth, thin-walled, hyaline, IKI-, CB-.

Remarks - Veluticeps is characterized by the dimitic hyphal system, usually encrusted pale to dark brown pseudocystidia, and more or less fusiform inamyloid basidiospores (Nakasone 1990b). It is phylogenetically included in the Gloeophyllaceae closely related to Chaetoderma and Boreostereum among others (Larsson 2007b).

Vesiculomyces E. Hagstr., Bot. Notiser 130: 53, 1977.

Type species - Thelephora citrina Pers., Mycol. eur. (Erlanga) 1: 136, 1822.

Basidiome resupinate, adnate, effused, hymenophore smooth to tuberculate. Hyphal system monomitic, hyphae with simple-septa. Cystidia (gloeocystidia) vesicular, with no guttulate content, SA-. Basidia narrowly clavate to cylindrical, with 4-sterigmata. Basidiospores ellipsoid to subglobose, with an evident apiculus, thin-walled, smooth, amyloid. 
Remarks - Vesiculomyces was described to include Gloeocystidiellum species (G. citrinum group in Eriksson \& Ryvarden 1976) with simple-septate hyphae, gloeocystidia SA- and smooth amyloid basidiospores (Hagström 1977). Wu (1996) includes Vesiculomyces as a synonym of Gloiothele (with gloeocystidia SA+) on the basis of the nuclear behaviour subnormal in both genera, and because according to Boidin (1958) some gloeocystidia SA+ can be found in some cultures of Vesiculomyces citrinus. However, molecular studies by Larsson \& Larsson (2003) and Miller et al. (2006) suggested that Vesiculomyces, although closely related, it is a sister group of Gloiothele and it must be considered as a separate genus.

Vitreoporus Zmitr., Folia Cryptogamica Petropolitana 6: 99, 2018.

Type species - Polyporus dichrous Fr., Observationes mycologicae 1: 125, 1815.

Basidiome of tyromycetoid habitus with two-layered context, hymenophore poroid with sterile or fertile dissepiments, gelatinose, cornescent. Upper context soft-fibrous, white or ivory, with black line near the tubes; tube layer citrine, honey-brown, or with lilaceous tints, cartilaginous. Hyphal system pseudodimitic. Generative hyphae with clamp connections, hyaline, often encrusted with granules, CB-. Pseudoskeletal hyphae thick-walled, yellowish, with singular clamps, CB+. Leptocystidia fusoid of hyphoid, intrahymenial. Basidia short-clavate, with central constriction, 4spored, with clamp at the base. Basidiospores allantoid, thin-walled, IKI-, CB-. Causing a white rot.

Remarks - According to Zmitrovich (2018), Gloeoporus Mont. is similar, but differs by thinner contextual/subicular layer, only slightly curved basidiospores, longer basidia, and simpleseptate or nodose-septate hyphae.

Vuilleminia Maire, Bull. Soc. mycol. Fr. 18(Suppl.): 81, 1902.

Type species - Thelephora comedens Nees, Syst. Pilze (Würzburg): 239, 1816.

Basidiome resupinate, effused, decorticant (only some species not decorticant), ceraceous to gelatinous when fresh, hymenophore smooth to more or less tuberculate, whitish, greyish, cream, reddish brown or with violaceous tints. Hyphal system monomitic, hyphae clamped, usually thinwalled, hyaline, dendrohyphidia present and usually numerous. Cystidia present or absent. Basidia long, utriform to clavate, pedunculate, sinuous, 4-spored and with a basal clamp. Basidiospores allantoid to ellipsoid, large (ab. 15-20 $\mu \mathrm{m}$ ), smooth, thin-walled, with oil drops, IKI-, CB-.

Remarks - Vuilleminia is easily to recognize by the decorticant basidiome (only a few species with no decorticant basidiome), the presence of hyaline dendrohyphae, clamped hyphae, large basidia and basidiospores (Maire 1902, Boidin et al. 1989, Gorjón 2008, 2009). It has a typical European distribution, with only few known records outside this area in North Africa, Asia and North America. The austral counterpart is referred to Australovuilleminia.

Waitea Warcup \& P.H.B. Talbot, Trans. Br. mycol. Soc. 45(4): 503, 1962.

Type species - Waitea circinata Warcup \& P.H.B. Talbot, Trans. Br. mycol. Soc. 45(4): 503, 1962.

Basidiome effused, thin, hypochnoid, smooth, white becoming ochraceous. Hyphal system monomitic, subhymenial hyphae thin-walled, hyaline, short-celled, somewhat swollen, often irregular, some convoluted branched, basal hyphae coloured, long-celled, straight, with somewhat thickened walls, all hyphae lacking clamp connexions. Cystidia absent. Basidia oblong to cylindrical often constricted about the middle, with 4-sterigmata. Basidiospores oblong to cylindrical, ventrally flattened, hyaline to ochraceous, smooth, thin-walled, sometimes with retraction septa, not producing secondary spores by replication, IKI-.

Remarks - The genus basically reminds Thanatephorus, but it is differentiated by the contorted hymenial hyphae branching and absence of repetition in the basidiospores (Warcup \& Talbot 1962). It is mainly tropical, with the type species isolated from soil (also known on Secale cereale straw in greenhouse). 
Wolfiporia Ryvarden \& Gilb., Mycotaxon 19: 141, 1984.

Type species - Poria cocos (Schwein.) Wolf, J. Elisha Mitchell Sci. Soc. 38: 134, 1922.

Basidiome annual, resupinate, pore surface white to ochraceous, pores circular to angular, context white to pale buff, firm to fibrous. Hyphal system dimitic, generative hyphae simpleseptate, thin- to thick-walled, some considerably inflated, skeletal hyphae thick-walled, hyaline to yellowish. Cystidiols fusoid present or absent. Basidia clavate, with 4-sterigmate, with a simple basal septum. Basidiospores ellipsoid to cylindrical, smooth, thin-walled, hyaline, IKI-.

Remarks - Wolfiporia is characterized by the simple-septate hyphae, some greatly inflated, presence of skeletals, and smooth basidiospores, negative in Melzer's reagent. According to Justo et al. (2017), Wolfiporia is nested with Laetiporus and Phaeolus in the Laetiporaceae remarking that the delimitation of Wolfiporia and Laetiporus needs further study.

Wrightoporia Pouzar, Ceska Mykol. 20: 173, 1966.

Type species - Poria lenta Overh. \& Lowe

Basidiome annual to perennial, membranous to cottony, resupinate to pileate, pores small to medium size, white to cream or grey, margin usually with rhizomorphs. Hyphal system dimitic, generative hyphae with clamps (some species with simple-septa), skeletal hyphae thick-walled to solid, dextrinoid in most and in the type species or not in some other species. Cystidia and gloeocystidia present in some species. Basidia clavate, with 4-sterigmate. Basidiospores globose to cylindrical, ornamented, weakly to strongly amyloid. On deciduous and coniferous wood, causing a white rot.

Remarks - Wrightoporia is characterized by the membranous to cottony basidiome, poroid hymenophore, usually dextrinoid vegetative hyphae, and amyloid ornamented basidiospores (Pouzar 1966a, Gilbertson \& Ryvarden 1987). The genus clearly belongs to the russuloid lineage even it seems to be polyphyletic (Larsson \& Larsson 2003). Some of the species previously classified in Wrightoporia have been transferred to Larssoniporia, Pseudowrightoporia, and Wrightoporiopsis (Chen et al. 2016).

Wrightoporiopsis Y.C. Dai, Jia J. Chen \& B.K. Cui, Persoonia 37: 31, 2015.

Type species - Wrightoporia neotropica Ryvarden, Karstenia 40: 156, 2000.

Basidiome annual or perennial, sessile, pileate, effused-reflexed or resupinate, soft to corky when fresh, corky to tough or brittle when dry. Pileal surface orange yellow to yellowish brown. Pore surface buff-yellow, ochraceous to olivaceous brown; pores small to tiny, round to angular. Context buff to cream, or pink to cinnamon-brown, cottony to hard corky. Tubes corky to tough, usually fragile. Hyphal system dimitic, generative hyphae with clamp connections, skeletal hyphae dextrinoid or IKI-. Gloeoplerous hyphae present or absent. Gloeocystidia present or absent. Cystidia present or absent. Basidiospores ellipsoid to subglobose, hyaline, thin- to thick-walled, finely asperulate, $\mathrm{IKI}+, \mathrm{CB}-$ or $\mathrm{CB}+$. Causing a white rot.

Remarks - Wrightoporiopsis is characterized by soft to corky basidiome when fresh, becoming tough and brittle upon drying small to tiny pores (5-10 per mm), corky to tough and usually fragile tubes, finely asperulate and amyloid basidiospores, and a tropical distribution. It is somewhat similar morphologically to Larssoniporia but differing in the smooth cystidia. The resulting phylogeny by Chen et al. (2016) do not resolve the phylogenetic status of some species of Wrightoporiopsis but the authors stated that they can be readily distinguished from Wrightoporia s.str. and other russuloid polypores by their poroid basidiomes, tough to brittle upon drying, and corky to tough, usually fragile tubes and a dimitic hyphal structure with clamped generative hyphae (Chen et al. 2016).

Xenasma Donk, Fungus, Wageningen 27: 25, 1957.

Type species - Corticium rimicola P. Karst., Hedwigia 35: 45, 1896.

Basidiome resupinate, adnate, ceraceous to gelatinous when fresh, membranaceous when dry, hymenophore smooth, margin indeterminate. Hyphal system monomitic, hyphae clamped, more or 
less glued together. Cystidia and cystidiols present. Basidia cylindrical to subclavate, pleural, usually with 4-sterigmata and a basal clamp. Basidiospores globose to cylindrical, warted to striate, inamyloid, sometimes weakly dextrinoid.

Remarks - Xenasma is characterized among the pleurobasidiate corticioid species by the ornamented basidiospores and the presence of cystidia. Molecular studies by Binder et al. (2005) place Xenasma in the russuloid clade, but the relationships with other genera seem unclear as pointed out by Larsson (2007b).

Xenasmatella Oberw., Sydowia 19 (1-6): 28, 1966.

Type species - Corticium subflavidogriseum Litsch., Annls mycol. 39(2/3): 127, 1941.

Remarks - A synonym of Phlebiella P. Karst.

Xenosperma Oberw., Sydowia 19 (1-6): 45, 1966.

Type species - Xenasma ludibundum D.P. Rogers \& Liberta, Mycologia 52(6): 902, 1962.

Basidiome resupinate, closely adnate to the substratum, inconspicuous, gelatinous, pruinose when dry. Hyphal system monomitic, hyphae with clamps, thin-walled, narrow, tortuous, not forming a subhymenium or a subiculum, basidia arising from only few hyphae. Cystidia absent. Basidia pleural, with 2-4-sterigmata, with a basal clamp. Basidiospores angular, tetrahedral, with some protuberances, hyaline, IKI-, CB-.

Remarks - Xenosperma is characterized by its thin and inconspicuous basidiome, pleurobasidia and angular basidiospores. Phylogenetic relationships in Xenosperma remain still unclear (Larsson 2007b).

Xerocarpus P. Karst., Revue mycol., Toulouse 3(no. 9): 22, 1881.

Type species - Thelephora alnea Fr., Syst. mycol. (Lundae) 1: 446, 1821.

Remarks - A latter homonym of Xerocarpus Guillermond \& Perrot 1832 (Fabaceae) and a synonym of Phanerochaete P. Karst.

Xylobolus P. Karst., Meddn Soc. Fauna Flora fenn. 6: 11, 1881.

Synonym - Acanthophysium (Pilát) G. Cunn.

Type species - Thelephora frustulata Pers., Syn. meth. fung. (Göttingen) 2: 577, 1801.

Basidiome annual or typically perennial, resupinate, effused or effuse-reflexed, coriaceous. Abhymenial surface tomentose, often concentrically zoned. Hymenophore smooth to tuberculate, pale coloured, often cracked. Hyphal system monomitic, but in some species seemingly dimitic; generative hyphae with simple septa, vertically arranged, thin- to thick-walled, hyaline to pale yellowish or brown. Pseudocystidia and acanthocystidia present. Cystidiols often present. Basidia clavate to cylindrical, with 4-sterigmata and with a simple basal septum. Basidiospores smooth, ellipsoid to cylindrical, thin-walled, amyloid.

Remarks - Xylobolus looks like Stereum but it causes a white pocket rot not known in the latter. Acanthophysium shares with Xylobolus simple-septate hyphae, smooth basidiospores, holocoenocytic nuclear behavior and phenoloxidase negative reaction and it was considered as synonym of Xylobolus by Wu et al. (2000). Acanthophysellum is phylogenetically closely related to Xylobolus but differs in the white rot, phenoloxidase positive reaction and nuclear behaviour astatocoenocytic.

Xylodon (Pers.) Gray, Nat. Arr. Brit. Pl. (London) 1: 649, 1821.

Basionym - Sistotrema sect. Xylodon Pers., Syn. meth. Fung. 552, 1801.

Type species - Odontia quercina Pers., Observ. mycol. (Lipsiae) 2: 17, 1800.

Basidiome resupinate, adnate, confluent, hymenophore smooth to grandinioid, tuberculate, raduloid or distinctly hydnoid, in some species poroid. Hyphal system monomitic, hyphae with clamps or rarely simple septate, thin- to commonly thick-walled, sometimes with a cyanophilous reaction. Cystidia variable, often not more than distinct hyphal ends, conical to more commonly 
capitate, rarely septate smooth or incrusted. Basidia mainly suburniform, thin-walled or basally thickened, usually with four sterigmata and a basal clamp. Basidiospores globose to ellipsoid, cylindrical or allantoid, smooth, thin- to rarely thick-walled, IKI-, sometimes with a slight cyanophilous reaction.

Remarks - Xylodon is applied here to those Hyphodontia s.l. species without well-defined cystidia (lagenocystidia, tubular cystidia, encrusted cystidia, etc.) and where only cystidial elements or more or less distinct hyphal ends are found. See additional comments in Hyphodontia. Xylodon is actually accepted for species with a variable hymenial configuration (smooth, grandinioid, aculeate, odontioid, poroid, ett.). See also Riebesehl \& Langer (2017).

Xylophagus Link, Mag. Gesell. naturf. Freunde, Berlin 3(1-2): 38, 1809.

Type species - Merulius destruens Pers., Syn. meth. fung. (Göttingen) 2: 496, 1801.

Remarks - A synonym of Serpula (Pers.) Gray.

Xylomyzon Pers., Mycol. eur. (Erlanga) 2: 26, 1825.

Type species - Merulius destruens Pers., Syn. meth. fung. (Göttingen) 2: 496, 1801.

Remarks - A synonym of Serpula (Pers.) Gray.

Yuchengia B.K. Cui \& K.T. Steffen, in Zhao, Cui \& Steffen, Nordic Jl Bot. 31(3): 333, 2013.

Type species - Trametes narymica Pilát, Bull. trimest. Soc. mycol. Fr. 51(3-4): 364, 1936.

Basidiome annual, resupinate. Pore surface cream to yellowish buff; pores angular, dissepiments thin, entire. Subiculum cream to buff, thin. Tubes concolorous with pore surface, hard corky. Hyphal system dimitic; generative hyphae hyaline, thin-walled, rarely branched, with clamp connections; skeletal hyphae dominant, hyaline, thick-walled with a wide to narrow lumen, usually unbranched, interwoven, weakly to distinctly amyloid in Melzer's reagent, acyanophilous, dissolving in $\mathrm{KOH}$. Cystidia absent, cystidioles variably present; basidia clavate, with four sterigmata and a basal clamp connection; basidioles dominant, in shape similar to basidia, but slightly smaller. Basidiospores ellipsoid, not truncate, hyaline, thick-walled, smooth, IKI-, CB+. Causing a white rot.

Remarks - Yuchengia is proposed to accommodate Perenniporia narymica (Pilát) Pouzar. It represents a monophyletic lineage within the core polyporoid clade based on sequences of the rDNA ITS and nLSU regions, and it is distinct from Perenniporia s.str. Yuchengia shares some characters with Perenniporia, both having thick-walled and cyanophilous basidiospores, but differs by having acyanophilous and amyloid skeletal hyphae dissolving in $\mathrm{KOH}$, and non-dextrinoid basidiospores (Zhao et al. 2013b).

Ypsilonidium Donk, Proc. K. Ned. Akad. Wet., Ser. C, Biol. Med. Sci. 75: 371, 1972.

Type species - Corticium sterigmaticum Bourdot, Rev. Sci. Bourb. Centr. Fr. 35(1): 4, 1922.

Remarks - A synonym of Thanatephorus Donk.

\section{Acknowledgements}

In the last years, many mycologists helped me in the search of bibliography, in the study of herbarium specimens or they contributed sending valuable specimens. I would like to especially thank those who welcomed me in their institutions (in chronological order of my research stays): Annarosa Bernicchia (Bologna, Italy), Leif Ryvarden (Oslo, Norway), Nils Hallenberg and KarlHenrik Larsson (Gothenburg, Sweden), Alina Greslebin and Mario Rajchenberg (Esquel, Argentina), Maria Aparecida de Jesus (Manaus, Brazil) and Juliano M. Baltazar (Porto Alegre, Brazil). Francisco Javier Hernández García (curator of herbarium SALA, University of Salamanca, Spain) managed most of the reference studied herbarium specimens. This text is intended to be updated regularly so any reader is grateful for their comments, criticisms, and contributions. 


\section{References}

Andreasen M, Hallenberg N. 2009 - A taxonomic survey of the Peniophoraceae. Synopsis Fungorum 26: 56-119.

Ariyawansa HA, Hyde KD, Jayasiri SC et al. 2015 - Fungal diversity notes 111-252 - taxonomic and phylogenetic contributions to fungal taxa. Fungal Diversity 75: 27-274.

Audet S. 2017a - Mushrooms nomenclatural novelties 1: 1.

Audet S. 2017b - Mushrooms nomenclatural novelties 2: 1 .

Audet S. 2017c - Mushrooms nomenclatural novelties 3: 1 .

Audet S. 2017d - Mushrooms nomenclatural novelties 4: 1 .

Audet S. 2017e - Mushrooms nomenclatural novelties 5: 1.

Audet S. 2017f - Mushrooms nomenclatural novelties 6: 1-2.

Audet S. 2017g - Mushrooms nomenclatural novelties 7: 1-2.

Audet S. 2017h - Mushrooms nomenclatural novelties 8: 1-2.

Audet S. 2017i - Mushrooms nomenclatural novelties 9: 1-2.

Audet S. 2017j - Mushrooms nomenclatural novelties 11: 1.

Baltazar JM, Gorjón SP, Pildain MB et al. 2015 - Acanthocorticium brueggemannii, a new corticioid genus and species related to cyphelloid fungi in the euagarics clade (Agaricales, Basidiomycota). Botany 93(8): 453-563.

Baltazar JM, Pildain MB, Gorjón SP et al. 2014 - Phylogenetic relationships of Hydnum peroxydatum support the synonymy of Hydnochaete with Hymenochaete (Hymenochaetaceae, Agaricomycetes). Mycologia 106(2): 323-327.

Baltazar JM, Silveira RMB da, Rajchenberg M. 2016 - Type studies of J. Rick's corticioid homobasidiomycetes (Agaricomycetes, Basidiomycota) housed in the Herbarium Anchieta (PACA). Phytotaxa 255(2): 101-132.

Berkeley MJ. 1873 - Australian fungi, received principally from Baron F. von Mueller and Dr. R. Schomburgk. Journal of the Linnean Society, Botany 13: 155-177.

Bernicchia A. 2005 - Polyporaceae s.l., Fungi Europaei 10. Ed. Candusso. Italia.

Bernicchia A, Gorjón SP. 2010 - Corticiaceae s.l. Fungi Europaei 12. Ed. Candusso. Italia. 1008 pp. Bernicchia A, Gorjón SP. 2020 - Polypores of the Mediterranean Region. Ed. Romar. Italia. 904 pp.

Bernicchia A, Gorjón SP, Nakasone KK. 2011 - Arrasia rostrata (Basidiomycota), a new corticioid genus and species from Italy. Mycotaxon 118: 257-264.

Bernicchia A, Vampola P, Prodi A et al. 2012 - A phylogenetic analysis of Antrodia s.l. based on nrDNA ITS sequences, with emphasis on rhizomorphic European species. Mycol. Progress 11: 93-100.

Binder M, Hibbett DS. 2002 - Higher level phylogenetic relationships of homobasidiomycetes (mushroom forming fungi) inferred from four rDNA regions. Molecular Phylogenetics and Evolution 22: 76-90.

Binder M, Hibbett DS. 2006 - Molecular systematics and biological diversification of Boletales. Mycologia 98: 971-981.

Binder M, Hibbett DS, Larsson KH et al. 2005 - The phylogenetic distribution of resupinate forms in the homobasidiomycetes. Systematics and Biodiversity 3: 113-157.

Binder M, Justo A, Riley R et al. 2013. - Phylogenetic and phylogenomic overview of the Polyporales. Mycologia 105: 1350-1373.

Binder M, Larsson KH, Matheny PB, Hibbett DS. 2010 - Amylocorticiales ord. nov. and Jaapiales ord. nov.: Early-diverging clades of Agaricomycetidae were dominated by corticioid forms. Mycologia 102(4): 865-80.

Bodensteiner P, Binder M, Agerer R et al. 2004 - Phylogenetic diversity of cyphelloid forms in the homobasidiomycetes. Molecular Phylogenetics and Evolution 33(2): 501-515.

Boidin J. 1958 - Hétérobasidiomycètes saprophytes et Homobasidiomycètes résupinés. V. Essai sur le genre Stereum Pers. ex S.F. Gray. Revue de Mycologie 23(3): 318-346. 
Boidin J. 1994 - Les Peniophoraceae del parties tempérées et froid de l'hémisphére nord. (Basidiomycotina). Bulletin mensuel de la Société Linnéenne de Lyon 63(9): 317-334.

Boidin J, Candoussau F, Gilles G. 1986 - Bambusicolous fungi from the Southwest of France II. Saprobic Heterobasidiomycetes, resupinate Aphyllophorales and Nidulariales. Transactions of the Mycological Society of Japan 27: 463-471.

Boidin J, Gilles G. 1989 - Les Corticiés pleurobasidiés (Basidiomycotina) en France. Cryptogamic Botany 1(1): 70-79.

Boidin J, Gilles G. 1998 - Contribution à l'étude des genres Dendrocorticium, Dendrodontia et Dentocorticium (Basidiomycotina). Cryptogamie, Mycologie 19(3): 181-202.

Boidin J, Gilles G. 2001 - Basidiomycètes Aphyllophorales de l'ile de Réunion XXIII Aleurodiscoideae. Bulletin de la Société Mycologique de France 117(3): 173-181.

Boidin J, Gilles G. 2002 - À propos du genre Lopharia sensu lato (Basidiomycètes, Aphyllophorales). Bulletin de la Société Mycologique de France 118(2): 91-115.

Boidin J, Lanquetin P. 1971 - Basidiomycetes Corticiaceae de la République Centroafricaine III. Le genre Hypochnicium Eriksson. Cahiers de La Maboké 9(2): 89-93.

Boidin J, Lanquetin P. 1983 - Les genres Vuilleminia et Corticium sensu stricto (Basidiomycètes) en France. Bulletin de la Société Mycologique de France 99: 269-279.

Boidin J, Lanquetin P. 1984 - Le genre Amylostereum (Basidiomycètes) intercompatibilités partielles entre espèces allopatriques. Bulletin de la Société Mycologique de France 100(2): 211-236.

Boidin J, Lanquetin P, Gilles G. 1989 - Une nouvelle espèce de Vuilleminia: V. coryli (Basidiomycotina). Bulletin de la Société Mycologique de France 105(2): 163-168.

Boidin J, Lanquetin P, Gilles G. 1997a - Le genre Gloeocystidiellum sensu lato (Basidiomycotina). Bulletin de la Société Mycologique de France 113(1): 1-80.

Boidin J, Lanquetin P, Gilles G. 1997b - Contribution à la connaissance du genre Asterostroma Massee 1889 (Basidiomycotina). Bulletin de la Société Mycologique de France 113(4): 269301.

Boidin J, Lanquetin P, Gilles G et al. 1985 - Contribution à la connaissance des Aleurodiscoideae à spores amyloides (Basidiomycotina, Corticiaceae). Bulletin de la Société Mycologique de France 101: 4: 333-367.

Boidin J, Mugnier J, Canales R. 1998 - Taxonomie moleculaire des Aphyllophorales. Mycotaxon 66: 445-491.

Boidin J, Terra P, Lanquetin P. 1968 - Contribution à la connaissance des caractères mycéliens et sexuel des genres Aleurodiscus, Dendrothele, Laeticorticium et Vuilleminia (Basidiomycètes, Corticiaceae). Bulletin de la Société Mycologique de France 84: 53-84.

Bondartsev AS. 1971 (1953) - The Polyporaceae of the European USSR and Caucasia. English translation: Israel program for Scientific translations, Jerusalem 1971, pp. 896.

Braun U. 2013 - (2210-2232) Proposals to conserve the teleomorph-typified name Blumeria against the anamorph-typified name Oidium and twenty-two teleomorph-typified powdery mildew species names against competing anamorph-typified names (Ascomycota: Erysiphaceae). Taxon 62(6): 1328-1331.

Bruns TD, Szaro TM, Gardes M et al. 1998 - A sequence database for identification of ectomycorrhizal Basidiomycetes by phylogenetic analysis. Molecular Ecology 7: 257-272.

Budington AB, Gilbertson RL. 1973 - Some Southwestern lignicolous Hymenomycetes of special interest. Southwestern Naturalist 17: 409-422.

Burdsall HH Jr. 1979 - Laetisaria (Aphyllophorales, Corticiaceae), a new genus for the teleomorph of Isaria fuciformis. Transactions of the British Mycological Society 72(3): 419-422.

Burdsall HH Jr. 1985 - A contribution to the taxonomy of the genus Phanerochaete. Mycologia Memoirs 10: 1-165.

Buyck B, Duhem B, Eyssartier G, Ducousso M. 2012 - Podoserpula miranda sp. nov. (Amylocorticiales, Basidiomycota) from New Caledonia. Cryptogamie Mycologie 33(4):453-461. 
Chen JJ, Cui BK. 2013 - Phlebiporia bubalina gen. et. sp. nov. (Meruliaceae, Polyporales) from Southwest China with a preliminary phylogeny based on rDNA sequences. Mycological Progress 13: 563-573.

Chen JJ, Cui BK, Dai YC. 2016 - Global diversity and molecular systematics of Wrightoporia s.l. (Russulales, Basidiomycota). Persoonia 37: 21-36.

Chen JJ, Shen LL. 2014 - Amylosporus succulentus sp. nov. (Russulales, Basidiomycota) evidenced by morphological characters and phylogenetic analysis. Cryptogamie Mycologie 35: 271-282.

Chen YY, Wu F, Wang M, Cui BK. 2017 - Species diversity and molecular systematics of Fibroporia (Polyporales, Basidiomycota) and its related genera. Mycological Progress 16(5): 521-533.

Corner EJH. 1976 - Further notes on cantharelloid fungi and Thelephora. Nova Hedwigia 27: 325342.

Cui BK, Li HJ, Ji X et al. 2019 - Species diversity, taxonomy and phylogeny of Polyporaceae (Basidiomycota) in China. Fungal Diversity 97: 137-392.

Cunningham GH. 1954 - Thelephoraceae of New Zealand. Transactions of the Royal Society of New Zealand 82: 271-327.

Dai LD, He SH. 2016 - New species and new records of Aleurodiscus s.l. (Basidiomycota) in China. Mycological Progress 15(7): 717-730.

Dai YC, Cui BK, Yuan SH et al. 2011 - Wood-inhabiting fungi in southern China 4. Polypores from Hainan Province. Annales Botanici Fennici 48(3): 219-231.

De Koker T, Nakasone KK, Haarhof J et al. 2003 - Phylogenetic relationships of the genus Phanerochaete inferred from the internal transcribed spacer region. Mycological Research 107: 1032-1040.

Decock C, Stalpers JA. 2006 - Studies in Perenniporia: Polyporus unitus, Boletus medulla-panis, the nomenclature of Perenniporia, Poria and Physisporus, and a note on European Perenniporia with a resupinate basidiome. Taxon 55(3): 759-778.

Dhingra GS. 2006 - Trimitiella gen. nov. (Basidiomycetes) from Eastern Himalaya, India. Mycotaxon 97: 125-128.

Dhingra GS, Priyanka. 2011a - Dendrophlebia (Agaricomycetes), a new corticioid genus from India. Mycotaxon 116: 157-160.

Dhingra GS, Priyanka. 2011b - Hallenbergia (Agaricomycetes), a new corticioid genus. Mycotaxon 118: 289-292.

Dhingra GS, Priyanka, Kaur J. 2012 - Radulomycetopsis (Agaricomycetes), a new corticioid genus from India. Mycotaxon 119: 133-136.

Dhingra GS, Singh Avneet P. 2006 - Repetobasidiopsis gen. nov. (Basidiomycetes) from Eastern Himalaya, India. Mycotaxon 97: 115-118.

Dhingra GS, Singh Avneet P. 2008 - Validation of Repetobasidiopsis and Trimitiella (Basidiomycetes). Mycotaxon 105: 421-422.

Diederich P. 1996 - The lichenicolous Heterobasidiomycetes. Bibliotheca Lichenologica. 61: 1198.

Diederich P, Lawrey JD. 2007 - New lichenicolous, muscicolous, corticolous and lignicolous taxa of Burgoa s.l. and Marchandiomyces s.l. (anamorphic Basidiomycota), a new genus for Omphalina foliacea, and a catalogue and a key to the non-lichenized, bulbilliferous basidiomycetes. Mycological Progress 6: 61-80.

Donk MA. 1931 - Revisie van de Nederlandse Heterobasidiomycetae en Homobasidiomycetae Aphyllophoraceae I. Mededelingen van de Nederlandse Mycologische Vereeniging 18-20: 67-200.

Donk MA. 1941 - Nomina generica conservanda and confusa for basidiomycetes (fungi). Bulletin of the Botanical Gardens Buitenzorg 17: 155-197.

Donk MA. 1954 - Notes on resupinate Hymenomycetes I. Reinwardtia 2: 425-434.

Donk MA. 1956 - Notes on resupinate Hymenomycetes III. Fungus 26: 3-24. 
Duhem B, Buyck B. 2011 - Meruliophana mahorensis gen. et sp. nov. de l'île de Mayotte (France Outre-mer). Cryptogamie Mycologie 32(2): 135-143.

Duhem B, Schultheis B. 2011 - Bourdotiella complicata gen. et sp. nov. de France. Cryptogamie Mycologie 32(3): 391-401.

Eriksson J. 1954 - Ramaricium n. gen., a corticioid member of the Ramaria group. Svensk Botanisk Tidskrift 48: 188-198.

Eriksson J, Hjortstam K. 1970 - Erythricium, a new genus of Corticiaceae (Basidiomycetes). Svensk Botanisk Tidskrift 64(2): 165-169.

Eriksson J, Hjortstam K, Ryvarden L. 1978 - The Corticiaceae of North Europe. Vol. 5. Mycoaciella - Phanerochaete. Fungiflora, Oslo, Norway.

Eriksson J, Hjortstam K, Ryvarden L. 1981 - The Corticiaceae of North Europe. Vol. 6. PhlebiaSarcodontia. Fungiflora, Oslo, Norway.

Eriksson J, Hjortstam K, Ryvarden L. 1984 - The Corticiaceae of North Europe. Vol. 7. Schizopora-Suillosporium. Fungiflora, Oslo, Norway.

Eriksson J, Ryvarden L. 1973 - The Corticiaceae of North Europe. Vol. 2. AlerurodiscusConfertobasidium. Fungiflora, Oslo, Norway.

Eriksson J, Ryvarden L. 1975 - The Corticiaceae of North Europe. Vol. 3. CoroniciumHyphoderma. Fungiflora, Oslo, Norway.

Eriksson J, Ryvarden L. 1976 - The Corticiaceae of North Europe. Vol. 4. HyphodermellaMycoacia. Fungiflora, Oslo, Norway.

Fiasson JL, Niemelä T. 1984 - The Hymenochaetales: a revision of European poroid taxa. Karstenia 24: 14-28.

Floudas D, Hibbett DS. 2015 - Revisiting the taxonomy of Phanerochaete (Polyporales, Basidiomycota) using a four gene dataset and extensive ITS sampling. Fungal Biology 119: 679-719.

Ghobad-Nejhad M, Dai YC. 2010 - Diplomitoporus rimosus is found in Asia and belongs to the Hymenochaetales. Mycologia 102(6): 1510-1517.

Ghobad-Nejhad M, Duhem B. 2014 - Novelties in the Corticiales: Vuilleminia nilsii sp. nov. and Dendrominia gen. nov. (Basidiomycota). Mycological Progress 13: 1-11.

Ghobad-Nejhad M, Hallenberg N. 2010 - Erythricium atropatanum sp. nov. (Corticiales) from Iran, based on morphological and molecular data. Mycological Progress 10(1): 61-66.

Ghobad-Nejhad M, Kotiranta H. 2007 - Re-evaluation of Radulomyces rickii, with notes on Phlebiella and Radulomyces (Corticiaceae, Basidiomycota). Mycotaxon 102: 101-111.

Ghobad-Nejhad M, Nilsson RH, Hallenberg N. 2010 - Phylogeny and taxonomy of the genus Vuilleminia (Basidiomycota) based on molecular and morphological evidence, with new insights into Corticiales. Taxon 59(5): 1519-1534.

Giachini AJ, Hosaka K, Nouhra E et al. 2010 - Phylogenetic relationships of the Gomphales based on nuc-25S-rDNA, mit-12S-rDNA, and mit-atp6-DNA combined sequences. Fungal Biology 114: 224-234.

Gilbertson RL, Blackwell M. 1988 - Some new or unusual corticioid fungi from the Gulf Coast region. Mycotaxon 33: 375-386.

Gilbertson RL, Nakasone KK. 2003 - New taxa of Hawaiian corticioid fungi are described with keys to Crustoderma, Radulomyces, and Scopuloides. Mycologia 95(3): 467-473.

Gilbertson RL, Ryvarden L. 1986 - North American Polypores. Abortiporus-Lindtneria 1: 1-433. Fungiflora, Oslo. Norway.

Gilbertson RL, Ryvarden L. 1987 - North American Polypores. Megasporoporia-Wrightoporia 2: 434-885. Fungiflora, Oslo. Norway.

Ginns J. 1976 - Corneromyces kinabalui, gen. nov., sp. nov. (Aphyllophorales, Coniophoraceae). Mycologia 68(5): 970-975.

Ginns J. 1978 - Leucogyrophana (Aphyllophorales): identification of species. Canadian Journal of Botany 56(16): 1953-1973. 
Ginns J. 1982 - A monograph of the genus Coniophora (Aphyllophorales, Basidiomycetes). Opera Botanica 61: 1-61.

Ginns J. 1984 - Griseoporia, a new genus for Hexagonia carbonaria (Polyporaceae). Mycotaxon 20(2): 559-565.

Ginns J. 1988 - New genera and species of lignicolous Aphyllophorales. Mycologia 80(1): 63-71.

Ginns J. 1998 - Genera of the North American Corticiaceae sensu lato. Mycologia 90: 1-35.

Gómez-Montoya N, Rajchenberg M, Robledo GL. 2017 - Aegis boa (Polyporales, Basidiomycota) a new neotropical genus and species based on morphological data and phylogenetic evidences. Mycosphere 8(6): 1261-1269.

Goranova G, Binder M, Hibbett DS. 2003 - Molecular phylogenetics indicate that the corticioid genus Dendrothele is highly polyphyletic. Inoculum 54: 22.

Gorjón SP. 2008 - Contribución al estudio Taxonómico, Corológico y Ecológico de los hongos Aphyllophorales s.l. y Gasterales s.l. presentes en los Ecosistemas del Parque Natural y Reserva de la Biosfera de "Las Batuecas-Sierra de Francia" (Salamanca, España). PhD Thesis. https://gredos.usal.es/handle/10366/20470

Gorjón SP. 2009 - Notas sobre el género Vuilleminia (Corticiaceae, Basidiomycota). Boletín de la Sociedad Micológica de Madrid 33: 81-89.

Gorjón SP. 2012 - Some species of Hyphodontia s.l. with encrusted cystidial elements. Mycosphere 3(4): 464-474.

Gorjón SP, Bernicchia A. 2010 - The genus Dendrothele (Basidiomycota) in Italy, an update with notes on European species. Nova Hedwigia 90(1-2): 233-250.

Gorjón SP, Greslebin AG, Rajchenberg M. 2011 - Notes on Amylocortiellum (Amylocorticiales, Basidiomycota), with some new combinations. Mycotaxon 116: 183-293.

Gorjón SP, Greslebin AG, Rajchenberg M. 2012 - Uncobasidium roseocremeum sp. nov. and other corticioid basidiomycetes from the Patagonian Andes of Argentina. Mycotaxon 121: 349-364.

Gorjón SP, Greslebin AG, Rajchenberg M. 2013 - The genus Aleurodiscus s.l. (Stereaceae, Russulales) in the Patagonian Andes. Mycological Progress 12: 91-108.

Gorjón SP, Hallenberg N. 2008 - New records of Sistotrema species (Basidiomycota) from the Iberian Peninsula. Sydowia 60: 205-212.

Greslebin AG, Rajchenberg M. 1999 - Corticioid Aphyllophorales (Basidiomycota) from the Patagonian Andes forests of Argentina 4. Nothocorticium patagonicum gen. et sp. nov. Mycotaxon 70: 371-376.

Greslebin AG, Rajchenberg M. 2000 - The genus Hyphodontia in the Patagonian Andes forests of Argentina. Mycologia 92: 1155-1165.

Greslebin AG, Nakasone KK, Rajchenberg M. 2004 - Rhizochaete, a new genus of phanerochaetoid fungi. Mycologia 96(2): 260-271.

Hagström E. 1977 - Vesiculomyces gen. nov. segregated from Gloeocystidiellum (Corticiaceae). Botaniska Notiser 130: 53-54.

Hallenberg N. 1980 - New taxa of Corticiaceae from N. Iran (Basidiomycetes). Mycotaxon 11(2): 447-475.

Hallenberg N. 1985 - The Lachnocladiaceae and Coniophoraceae of North Europe. 96 pp. Fungiflora, Oslo, Norway.

Hallenberg N, Hjortstam K. 1988 - Studies in Corticiaceae (Basidiomycetes). New species and new combinations. Mycotaxon 31 (2): 439-443.

Hallenberg N, Hjortstam K, Ryvarden L. 1985 - Pirex genus nova (Basidiomycetes, Corticiaceae). Mycotaxon 24: 287-291.

Hallenberg N, Parmasto P. 1998 - Phylogenetic studies in species of Corticiaceae growing on branches. Mycologia 90: 640-654.

Hallenberg N, Yurchenko E, Ghobad-Nejhad, M. 2010 - Peniophora pseudonuda is a synonym of P. laeta. Mycotaxon 112. 153-162. 
He SH, Vlasák J, Dai YC. 2014 - Hispidaedalea gen. nov. and Griseoporia taiwanense sp. nov. (Gloeophyllales, Basidiomycota) based on morphological and molecular characters. Mycological Progress 13: 833-839.

Hibbett DS, Binder M. 2002 - Evolution of complex fruiting-body morphologies in homobasidiomycetes. Proceedings of the Royal Society of London 269: 1963-1969.

Hibbett DS, Pine EM, Langer E et al. 1997 - Evolution of gilled mushrooms and puffballs inferred from ribosomal DNA sequences. Proceedings of the National Academy of Sciences, USA 94: 12002-12006.

Hibbett DS, Thorn RG. 2001 - Homobasidiomycetes. In: McLaughlin DJ, McLaughlin EJ, Lemke P (eds), The Mycota. Vol. VII. Part B., Systematics and Evolution. Springer-Verlag, Berlin, pp. 121-168.

Hjortstam K. 1981 - Notes on Corticiaceae. IX. Three new combinations in Hypochniciellum. Mycotaxon 13(1): 124-126.

Hjortstam K. 1983 - Studies in tropical Corticiaceae (Basidiomycetes). V. Specimens from East Africa collected by L. Ryvarden. Mycotaxon 17: 555-572.

Hjortstam K. 1987a - A check-list to genera and species of corticioid fungi (Hymenomycetes). Windahlia 17: 55-85.

Hjortstam K. 1987b - Studies in tropical Corticiaceae (Basidiomycetes) VII. Specimens from East Africa collected by L. Ryvarden. II. Mycotaxon 28(1): 19-37.

Hjortstam K. 1991 - Athelopsis instead of Pteridomyces (Corticiaceae, Basidiomycetes). Mycotaxon 42: 149-154.

Hjortstam K. 1995 - Two new genera and some new combinations of corticioid fungi (Basidiomycotina, Aphyllophorales) from tropical and subtropical areas. Mycotaxon 54: 183193.

Hjortstam K. 1998 - A checklist to genera and species of corticioid fungi (Basidiomycotina, Aphyllophorales). Windahlia 23: 1-54.

Hjortstam K. 1999 - New corticioid taxa from Brazil, with a brief discussion on Hydnum setulosum (Basidiomycotina). Kew Bulletin 54(3): 755-761.

Hjortstam K. 2001 - Tublicrinaceae - a survey of the genera and species. Windahlia 24: 1-14.

Hjortstam K, Bononi VLR. 1987 - A contribution to the knowledge of Corticiaceae s.l. (Aphyllophorales) in Brazil Mycotaxon 28(1): 1-15.

Hjortstam K, Larsson KH. 1976 - Pseudoxenasma, a new genus of Corticiaceae (Basidiomycetes). Mycotaxon 4(1): 307-311.

Hjortstam K, Larsson KH. 1977 - Notes on Corticiaceae (Basidiomycetes). Mycotaxon 5(2): 475480.

Hjortstam K, Larsson KH. 1978 - Notes on Corticiaceae (Basidiomycetes) II. Mycotaxon 7(1): 117-124.

Hjortstam K, Larsson KH. 1982 - Notes on Corticiaceae (Basidiomycetes). Mycotaxon 14: 69-74.

Hjortstam K, Larsson KH. 1987 - Additions to Phlebiella (Corticiaceae, Basidiomycetes), with notes on Xenasma and Sistotrema. Mycotaxon 29: 315-319.

Hjortstam K, Larsson KH, Ryvarden L. 1987 - The Corticiaceae of North Europe 1. Introduction and keys. Fungiflora, Oslo, Norway.

Hjortstam K, Larsson KH, Ryvarden L. 1988 - The Corticiaceae of North Europe 8. PhlebiellaThanatephorus-Ypsilonidium. Fungiflora, Oslo, Norway.

Hjortstam K, Ryvarden L. 1978 - Notes on Corticiaceae (Basidiomycetes) III. Mycotaxon 7(2): 407-410.

Hjortstam K, Ryvarden L. 1979a - Notes on Corticiaceae (Basidiomycetes) IV. Mycotaxon. 9(2): 505-519.

Hjortstam K, Ryvarden L. 1979b - Notes on Corticiaceae (Basidiomycetes) V. Mycotaxon 10(1): 201-209.

Hjortstam K, Ryvarden L. 1980a - Studies in tropical Corticiaceae (Basidiomycetes) I. Mycotaxon 10(2): 269-287. 
Hjortstam K, Ryvarden L. 1980b - Studies in tropical Corticiaceae (Basidiomycetes) II. Mycotaxon 12(1): 168-184.

Hjortstam K, Ryvarden L. 1986 - Some new and noteworthy fungi (Aphyllophorales, Basidiomycetes) from Iguazu, Argentina. Mycotaxon 25(2): 539-567.

Hjortstam K, Ryvarden L. 1990 - Lopharia and Porostereum (Corticiaceae). Synopsis Fungorum 4: $1-68$.

Hjortstam K, Ryvarden L. 2001 - Corticioid species (Basidiomycotina, Aphyllophorales) from Colombia III. Mycotaxon 79: 189-200.

Hjortstam K, Ryvarden L. 2002a - Studies in tropical corticioid fungi (Basidiomycotina, Aphyllophorales) Alutaceodontia, Botryodontia, Hyphodontia s.str. and Kneiffiella. Synopsis Fungorum 15: 7-17.

Hjortstam K, Ryvarden L. 2002b - Australicium (Basidiomycotina, Aphyllophorales) a new genus for Corticium singulare G. Cunn. Synopsis Fungorum 15: 18-21.

Hjortstam K, Ryvarden L. 2002c - Leptocorticium, a new genus among the corticioid fungi (Basidiomycotina, Aphyllophorales). Synopsis Fungorum 15: 22-25.

Hjortstam K, Ryvarden L. 2004a - Some new and notheworthy corticioid fungi (Basidiomycotina, Aphyllophorales) from Japan. Synopsis Fungorum 18: 8-13.

Hjortstam K, Ryvarden L. 2004b - Reevalution of Nodotia Hjortstam (Corticioid fungi, Basidiomycotina, Aphyllophorales). Synopsis Fungorum 18: 17-19.

Hjortstam K, Ryvarden L. 2004c - Some new tropical genera and species of corticioid fungi (Basidiomycotina, Aphyllophorales). Synopsis Fungorum 18: 20-32.

Hjortstam K, Ryvarden L. 2005 - New taxa and new combinations in tropical corticioid fungi (Basidiomycotina, Aphyllophorales). Synopsis Fungorum 20: 33-41.

Hjortstam K, Ryvarden L. 2007 - Studies in corticioid fungi from Venezuela III (Basidiomycotina, Aphyllophorales). Synopsis Fungorum 23: 56-107.

Hjortstam K, Ryvarden L. 2008 - Corticioid species (Basidiomycotina, Aphyllophorales) from Colombia IV. Synopsis Fungorum 25: 28-37.

Hjortstam K, Ryvarden L. 2009a - A preliminary checklist of Aphyllophorales from the Seychelles. Synopsis Fungorum 26: 10-23.

Hjortstam K, Ryvarden L. 2009b - A checklist of names in Hyphodontia sensu stricto - sensu lato and Schizopora with new combinations in Lagarobasidium, Lyomyces, Kneiffiella, Schizopora, and Xylodon. Synopsis Fungorum 26: 33-55.

Hjortstam K, Ryvarden L. 2010a - Athelocystis capitata, a new genus and species from Brazil, with brief notes on Athelopsis, Corticioid fungi (Basidiomycota). Synopsis Fungorum 27: 20-25.

Hjortstam K, Ryvarden L. 2010b - Phanerodontia and Phaneroites, two corticioid taxa (Basidiomycotina) proposed from tropical areas. Synopsis Fungorum 27: 26-33.

Hjortstam K, Spooner BM, Oldridge SG. 1990 - Some Aphyllophorales and Heterobasidiomycetes from Sabah, Malaysia. Kew Bulletin 45: 303-322.

Hjortstam K, Stalpers JA. 1982 - Notes on Corticiaceae (Basidiomycetes) XI. Boidinia, a new genus segregated from Gloeocystidiellum. Mycotaxon 14(1): 75-81.

Hjortstam K, Telleria MT. 1990 - Columnocystis, a synonym of Veluticeps. Mycotaxon 37: 53-56.

Höhnel Fv, Litschauer V. 1907 - Beiträge zur Kenntnis der Corticieen: II. Sitzungsberichte der Kaiserlichen Akademie der Wissenschaften, Math.-naturw. Klasse, Abt. I. 116: 739-852.

Holubová-Jechová V. 1969 - New species of the genus Oidium Link ex Fr. emend. Linder. Ceská Mykologie 23(4): 209-221.

Holubová-Jechová V. 1976 - Haplotrichum Link instead of Oidium Link, a necessary nomenclatural change. Ceská Mykologie 30(1): 3-4.

Hosaka K, Bates ST, Beever RE et al. 2006 - Molecular phylogenetics of the gomphoid-phalloid fungi with an establishment of the new subclass Phallomycetidae and two new orders. Mycologia 98(6): 949-959.

Jahn H. 1971 - Steroide Pilze in Europa, Stereaceae Pil. Emend. Parm. u. a., Hymenochaete. Westfälische Pilzbriefe 8: 69-176. 
Jarosch M, Besl H. 2001 - Leucogyrophana, a polyphyletic genus of the order Boletales (Basidiomycetes). Plant Biology 3(4): 443-448.

Ji X, Wu DM, Liu S et al. 2019 - Crassisporus gen. nov. (Polyporaceae, Basidiomycota) evidenced by morphological characters and phylogenetic analyses with descriptions of four new species. MycoKeys 57: 61-84.

Jülich W. 1969 - Über die Gattungen Piloderma gen. nov. und Subulicystidium Parm. (Corticiaceae, Aphyllophorales, Basidiomycetes). Berichte der Deutschen Botanischen Gesellschaft 81: 414-421.

Jülich W. 1972 - Monographie der Atheliae (Corticiaceae, Basidiomycetes). Willdenowia, Beih 7: $1-283$.

Jülich W. 1974a - The genera of the Hyphodermoideae (Corticiaceae). Persoonia 8(1): 59-97.

Jülich W. 1974b - On Scotoderma and Phlyctibasidium, two new genera of lower basidiomycetes with resupinate basidiocarps. Proceedings van de Koninklijke Nederlanse Akademie van Wetenschappen Section C 77(2): 149-156.

Jülich W. 1975 - Studies in resupinate Basidiomycetes - III. Persoonia 8(3): 291-305.

Jülich W. 1976a - Studies in resupinate Basidiomycetes - IV. Persoonia 8(4): 431-442.

Jülich W. 1976b - Studies in hydnoid fungi - I. Persoonia 8(4): 447-458.

Jülich W. 1978a - On some Aphyllophorales from Australia. Persoonia 9(4): 453-472.

Jülich W. 1978b - Studies in resupinate Basidiomycetes - V. Some new genera and species. Persoonia 10(1): 137-140.

Jülich W. 1979 - Studies in resupinate Basidiomycetes - VI. On some new taxa. Persoonia 10(3): 325-336.

Jülich W. 1981 - Higher taxa of Basidiomycetes. Bibliotheca Mycologica 85:1-485.

Jülich W. 1982 - Studies in resupinate Basidiomycetes - VII. International Journal of Mycology and Lichenology 1(1): 27-37.

Jülich W. 1984 - Notes on Subulicystidium longisporum (Pat.) Parm. International Journal of Mycology and Lichenology 1(3): 281-282.

Jülich W, Stalpers JA. 1980 - The resupinate non-poroid Aphyllophorales of the temperate northern hemisphere. North-Holland Publishing Company.

Junghuhn FW. 1840 - Nova genera et species plantarum florae javanicae. Tijdschrift voor Natuurlijke Geschiedenis en Physiologie 7: 285-317.

Justo A, Hibbett DS. 2011 - Phylogenetic classification of Trametes (Basidiomycota, Polyporales) based on a five-gene dataset. Taxon 60 (6): 1567-1583.

Justo A, Miettinen O, Floudas D, et al. 2017 - A revised family-level classification of the Polyporales (Basidiomycota). Fungal Biology 121(9): 798-824.

Karasiński D. 2013 - Lawrynomyces, a new genus of corticioid fungi in the Hymenochaetales. Acta Mycologica (Warszawa) 48(1): 5-11.

Karasiński D. 2014 - Mushrooms nomenclatural novelties 140: 1.

Karasiński D, Niemelä T. 2016 - Anthoporia, a new genus in the Polyporales (Agaricomycetes). Polish Botanical Journal 61(1): 7-14.

Katz B. 1974 - Ambivina filobasidia B. Katz gen. et sp. nov. Nova Hedwigia 25: 811-814.

Kim SY, Jung HS. 2000 - Phylogenetic relationships of the Aphyllophorales inferred from sequence qnalysis of Nuclear Small Subunit Ribosomal DNA. The Journal of Microbiology 38(3): 122-131.

Ko KS, Jung HS, Ryvarden L. 2001 - Phylogenetic relationships of Hapalopilus and related genera inferred from mitochondrial small subunit ribosomal DNA sequences. Mycologia 93: 270276.

Kõljalg U. 1996 - Tomentella (Basidiomycota) and related genera in Temperate Eurasia. Synopsis Fungorum 9: 1-213.

Kotiranta H, Hjortstam K, Miettinen O, Kulju M. 2007 - Tubulicrinopsis gen. novum (Basidiomycota, Aphyllophorales) and notes on Amauromyces pallidus. Annales Botanica Fennica 44: 128-134. 
Kotiranta H, Kulju M, Miettinen O. 2017 - Caudicicola gracilis (Polyporales, Basidiomycota), a new polypore species and genus from Finland. Ann. Bot. Fenn. 54: 159-167.

Kotiranta H, Larsson KH, Saarenoksa R, Kulju M. 2011 - Tretomyces gen. novum, Byssocorticium caeruleum sp. nova, and new combinations in Dendrothele and Pseudomerulius (Basidiomycota). Annales Botanici Fennici 48: 37-48.

Kotiranta H, Saarenoksa R. 2002 - New combinations in Irpex (Aphyllophorales, Basidiomycetes). Polish Botanical Journal 47(2): 103-107.

Kotlába F. 1988 - Correct name for Cytidiella melzeri Pouz. Ceská Mykol. 42: 239.

Kotlába F, Pouzar S. 1973 - Donkioporia Kotl. \& Pouz., a new genus for Poria megalopora (Pers.) Cooke. Persoonia 7: 213-216.

Kuntze O. 1898 - Revisio generum plantarum 3: 1-576.

Langer E. 1994a - Die Gattung Hyphodontia John Eriksson. Bibliotheca Mycologica 154, 298 pp.

Langer E. 2002 - Phylogeny of Non-Gilled and Gilled Basidiomycetes: DNA Sequence Inference. Ultrastructure and Comparative Morphology. Habilitationsschrift, Tübingen University, Tübingen.

Langer G. 1994b - Die gattung Botryobasidium Donk (Corticiaceae, Basidiomycetes). Bibliotheca Mycologica 158, 459 pp.

Larsen MJ. 1974 - A contribution to the taxonomy of the genus Tomentella. Mycologia Memoirs 4: $1-145$.

Larsen MJ, Cobb-Poulle LA. 1990 - Phellinus (Hymenochaetaceae) a survey of the world taxa. Synopsis Fungorum 3: 1-206.

Larsen MJ, Gilbertson RL. 1974 - Dendrocorticium and Dentocorticium gen. nov. (Aphyllophorales, Corticiaceae) as segregates from Laeticorticium. Norwegian Journal of Botany 21: 223-226.

Larsen MJ, Zak B. 1978 - Byssoporia gen. nov.: taxonomy of the mycorrhizal fungus Poria terrestris. Canadian Journal of Botany 56(9): 1122-1129.

Larsson E, Larsson KH. 2003 - Phylogenetic relationships of russuloid basidiomycetes with emphasis on aphyllophoralean taxa. Mycologia 95: 1037-1065.

Larsson KH. 1992 - The Genus Trechispora (Corticiaceae, Basidiomycetes). Dissertation. Universität Göteborg, Schweden.

Larsson KH. 2007a - Molecular phylogeny of Hyphoderma and the reinstatement of Peniophorella. Mycological Research 111: 186-195.

Larsson KH. 2007b - Re-thinking the classification of corticioid fungi. Mycological Research 111(9): 1040-1063.

Larsson KH, Hjortstam K. 1974 - Luellia, a new genus in the Corticiaceae (Basidiomycetes). Svensk botanisk Tidskrift 68(1): 57-63.

Larsson KH, Larsson E, Kõljalg U. 2004 - High phylogenetic diversity among corticioid Homobasidiomycetes. Mycological Research 108(9): 983-1002.

Larsson KH, Parmasto E, Fischer M et al. 2006 - Hymenochaetales: a molecular phylogeny of the hymenochaetoid clade. Mycologia 98: 926-936.

Lawrey JD, Binder M, Diederich P et al. 2007 - Phylogenetic diversity of lichen-associated homobasidiomycetes. Molecular Phylogenetics and Evolution 44: 778-789.

Lawrey JD, Lücking R, Sipman HJM, et al. 2009 - High concentration of basidiolichens in a single family of agaricoid mushrooms (Basidiomycota: Agaricales: Hygrophoraceae). Mycological Research 113(10): 1154-1171.

Leal-Dutra CA, Neves MA, Griffith GW et al. 2018 - Reclassification of Parapterulicium Corner (Pterulaceae, Agaricales), contributions to Lachnocladiaceae and Peniophoraceae (Russulales) and introduction of Baltazaria gen. nov. MycoKeys 37: 39-56.

Lee JS, Kim C. Lim YW. 2008 - Irpex hacksungii sp. nov. (Polyporaceae) from Korea. Mycotaxon 106: 423-429.

Lemke PA. 1964a - The genus Aleurodiscus (sensu stricto) in North America. Canadian Journal of Botany. 42: 213-282. 
Lemke PA. 1964b - The genus Aleurodiscus (sensu lato) in North America. Canadian Journal of Botany 42: 723-768.

Li HJ, Cui BK. 2013 - Taxonomy and phylogeny of the genus Megasporoporia and its related genera. Mycologia 105(2): 368-383.

Li HJ, Cui BK, Dai YC. 2014 - Taxonomy and multi-gene phylogeny of Datronia (Polyporales, Basidiomycota). Persoonia 32: 170-182.

Lim YW. 2001 - Systematic study of corticioid fungi based on molecular sequence analyses. PhD thesis, Soeul National University, Seoul.

Lim YW, Jung HS. 2003 - Irpex hydnoides, sp. nov. is new to science, based on morphological, cultural and molecular characters. Mycologia 95(4): 694-699.

Liu SL, Zhao Y, Dai YC, Nakasone KK, He SH. 2017 - Phylogeny and taxonomy of Echinodontium (Basidiomycota, Russulales) and related genera. Mycologia 109(4): 568-577.

Lücking R, Moncada B. 2017 - Dismantling Marchandiomphalina into Agonimia (Verrucariaceae) and Lawreymyces gen. nov. (Corticiaceae): setting a precedent to the formal recognition of thousands of voucherless fungi bases on type sequences. Fungal Diversity 84: 119-138.

Ma X, Zhao CL. 2019 - Crepatura ellipsospora gen. et sp. nov. in Phanerochaetaceae (Polyporales, Basidiomycota) bearing a tuberculate hymenial surface. Mycological Progress 18(6): 785793.

Maas Geesteranus RA. 1974 - Studies in the genera Irpex and Steccherinum. Persoonia 7(4): 443581.

Maekawa N, Suhara H, Kinjo K et al. 2005 - Haloaleurodiscus mangrovei gen. sp. nov. (Basidiomycota) from mangrove forests in Japan. Mycological Research. 109(7): 825-832.

Maire R. 1902 - Recherches cytologiques et taxonomiques sur les basidiomycètes. Bulletin de la Société Mycologique de France 18(Suppl.): 1-212.

Malençon G, Bertault R. 1963 - Lenzitopsis oxycedri Malençon \& Bertault, genre nouveau et espéce nouvelle d'Aphyllophorales a spores colorees. Bulletin de la Société Mycologique de France 79(1): 75-82.

Martin GW. 1949 - The genus Ceracea Cragin. Mycologia 41: 77-86.

Martín MP, Raidl S, Telleria MT. 2004 - Molecular analyses confirm the relationship between Stephanospora caroticolor and Lindtneria trachyspora. Mycotaxon 90 (1): 133-140.

Martini EC, Trichiès G. 2004 - Trechinothus smardae gen. et comb. nov., un champignon corticioïde à chlamydospores moriformes. Mycotaxon 90(2): 261-269.

Marvanová L, Stalpers JA. 1987 - The genus Taeniospora and its teleomorphs. Transactions of the British Mycological Society 89(4): 489-498.

Massee G. 1893 - British fungus flora 3. London. George Bells \& Sons.

McAlpine D. 1906 - A new hymenomycete - the so-called Isaria fuciformis Berk. Annales Mycologici 4(6): 541-551.

Miettinen O, Larsson KH. 2011 - Sidera, a new genus in Hymenochaetales with poroid and hydnoid species. Mycological progress 10(2): 131-141.

Miettinen O, Niemelä T, Ryvarden L. 2007 - A new polypore Irpex cremicolor described from North Europe. Mycotaxon 102: 415-424.

Miettinen O, Rajchenberg M. 2012 - Obba and Sebipora, new polypore genera related to Cinereomyces and Gelatoporia (Polyporales, Basidiomycota). Mycological Progress 11: 131147.

Miettinen O, Ryvarden L. 2016 - Polypore genera Antella, Austeria, Butyrea, Citripora, Metuloidea and Trulla (Steccherinaceae, Polyporales). Ann. Bot. Fennici 53: 157-172.

Miettinen O, Spirin V, Vlasák J et al. 2016 - Polypores and genus concepts in Phanerochaetaceae (Polyporales, Basidiomycota). Mycokeys. 17:1-46.

Miller SL, Larsson E, Larsson KH et al. 2006 - Perspectives in the new Russulales. Mycologia 98: 960-970.

Moncalvo JM, Nilsson RH, Koster B et al. 2006 - The cantharelloid clade: dealing with incongruent gene trees and phylogenetic reconstruction methods. Mycologia 98: 937-948. 
Moreno G, Blanco MN, Olariaga I, Checa J. 2007 - Climacodon pulcherrimus a badly known tropical species, present in Europe. Cryptogamie Mycologie 28(1): 3-11.

Murrill WA. 1907 - Polyporaceae, Part 1. North American Flora. 9(1): 1-72.

Nakasone KK. 1984 - Taxonomy of Crustoderma (Aphyllophorales, Corticiaceae). Mycologia 76(1): 40-50.

Nakasone KK. 1990a - Cultural studies and identification of wood-inhabiting Corticiaceae and selected Hymenomycetes from North America. Mycologia Memoir 165: 1-412.

Nakasone KK. 1990b - Taxonomic study of Veluticeps (Aphyllophorales). Mycologia 82(5): 622641.

Nakasone KK. 1996 - Morphological and molecular studies on Auriculariopsis albomellea and Phlebia albida and a reassessment of A. ampla. Mycologia 88: 762-775.

Nakasone KK. 1997 - Studies in Phlebia. Six species with teeth. Sydowia 49(1): 49-79.

Nakasone KK. 2002 - Mycoaciella, a synonym of Phlebia. Mycotaxon 81: 477-490.

Nakasone KK. 2003 - Type studies of resupinate hydnaceous Hymenomycetes described by Patouillard. Cryptogamie Mycologie 24(2): 131-145.

Nakasone KK. 2004 - Morphological studies in Veluticeps, Pileodon and related genera. Sydowia 56(2): 258-280.

Nakasone KK. 2009 - Type studie of corticioid Hymenomycetes (Basidiomycota) with aculei. Sydowia 61(2): 273-285.

Nakasone KK. 2011 - Boidinella gen. nov. (Cantharellales, Basidiomycota). Cryptogamie Mycologie 32(2): 191-197.

Nakasone KK. 2013 - Taxonomy of Epithele (Polyporales, Basidiomycota). Sydowia 65: 59-112.

Nakasone KK. 2015 - Taxonomic studies in Chrysoderma, Corneromyces, Dendrophysellum, Hyphoradulum, and Mycobonia. Mycotaxon 130: 369-397.

Nakasone KK, Burdsall HH Jr. 1984 - Merulius, a synonym of Phlebia. Mycotaxon 21: 241-246.

Nakasone KK, Burdsall HH Jr. HH. 2012 - Tsugacorticium kenaicum (Hymenochaetales, Basidiomycota), a new corticioid genus and species from Alaska. North American Fungi 7(1): 1-9.

Nakasone KK, Hibbett DS, Goranova G. 2009 - Neocampanella, a new corticioid fungal genus, and a note on Dendrothele bispora. Botany 87(9): 875-882.

Nakasone KK, Linder DL. 2012 - Taxonomy of Pseudolagarobasidium (Polyporales, Basidiomycota). Fungal Diversity 55: 155-169.

Nielsen C, Williams DW, Hajek AE. 2009 - Putative source of the invasive Sirex noctilio fungal symbiont, Amylostereum areolatum, in the eastern United States and its association with native siricid woodwasps. Mycological Research 113: 1242-1253.

Niemelä T. 2016 - Suomen käävät. Norrlinia 31: 1-432.

Niemelä T, Kinnunen J, Larsson KH, et al. 2005 - Genus revisions and new combinations of some North European polypores. Karstenia 45(2): 75-80.

Niemelä T, Larsson KH. 2007 - Anomoloma, a new genus separated from Anomoporia on the basis of decay type and nuclear rDNA sequence data. Mycotaxon 100: 305-317.

Niemelä T, Miettinen O, Manninen O. 2012 - Aurantiporus priscus (Basidiomycota), a new polypore from old fallen conifer trees. Annales Botanici Fennici 49(3): 201-205.

Nilsson RH, Larsson KH, Larsson E, Kõljalg U. 2006 - Fruiting body-guided molecular identification of root-tip mantle mycelia provides strong indications of ectomycorrhizal associations in two species of Sistotrema (Basidiomycota). Mycol Res. 110:1426-32.

Nobles MK. 1967 - Conspecifity of Basidioradulum (Radula) radula and Corticium hydnans. Mycologia 59: 192-211.

Núñez M, Ryvarden L. 1997 - The genus Aleurodiscus (Basidiomycotina). Synopsis Fungorum 12:1-164.

Oberwinkler F. 1965 - Primitive Basidiomyceten. Revision einer Formenkreis von Basidienpilze mit plastischer Basidie. Sydowia, Ann Mycol Ser II 19: 1-77.

Oberwinkler F. 1966 - Die Gattung Tubulicrinis Donk s.l. (Corticiaceae). Z Pilzk 31: 12-48. 
Oberwinkler F. 1970 - Die Gattungen der Basidiolichenen. Deutsche Botanische Gesellschaft Neue Folge 4:139-169.

Oberwinkler F. 1977 - Das neue System der Basidiomyceten. In: Frey W, Hurka H, Oberwinkler F, eds. Beiträge zur Biologie der niederen Pflanzen. Stuttgart, New York: Gustav Fischer Verlag. p 59-104.

Oberwinkler F, Langer E, Burdsall HH Jr, Tschen J. 1990 - Heteroacanthella: a new genus in the Tulasnellales. Transactions of the Mycological Society of Japan 31: 207-213.

Ortiz-Santana B, Lindner DL, Miettinen O, et al. 2013 - A phylogenetic overview of the Antrodia clade (Basidiomycota, Polyporales). Mycologia 105: 1391-1411.

Parmasto E. 1959 - On the distribution of some rare species of Polyporaceae. Eesti NSV Teaduste Akadeemia Toimetised 8: 266-278.

Parmasto E. 1967 - Corticiaceae U.R.S.S. IV. Descriptiones taxorum novarum. Combinationes novae. Eesti NSV Teaduste Akadeemia Toimetised 16: 377-394.

Parmasto E. 1968 - Conspectus Systematis Corticiacearum. 1-261. Tartu; Institutum Zoologicum et Botanicum Academiae Scientiarum RPSS Estonicae.

Parmasto E, Hallenberg N. 2000 - A taxonomic study of phlebioid fungi (Basidiomycota). Nordic Journal of Botany 20: 105-118.

Parmasto E, Nilsson RH, Larsson KH. 2004 - Cortbase version 2 - extensive updates of a nomenclatural database for corticioid fungi (Hymenomycetes). Phyloinformatics 5: 1-7.

Petersen RH. 1971 - A new genus segregated from Kavinia Pilát. Ceská Mykologie 25(3): 129_ 134.

Piątek M. 2004 - Cabalodontia (Meruliaceae), a novel genus for five fungi previously placed in Phlebia. Polish Botanical Journal 49(1): 1-3.

Pouzar Z. 1958 - Nova genera macromycetum II. Ceská Mykologie 12(1): 31-36.

Pouzar Z. 1959 - New genera of higher fungi III. Ceská Mykologie 13(1): 10-19.

Pouzar Z. 1966a - Studies in the taxomomy of the Polypores I. Ceská Mykologie 20(3): 171-177.

Pouzar Z. 1966b - Studies in the taxonomy of polypores II. Folia Geobotanica et Phytotaxonomica 1(4): 356-375.

Pouzar Z. 1983 - Taxonomic studies in resupinate fungi II. Ceská Mykologie 37(4): 206-211.

Rajchenberg M. 1996 - Polypores (Basidiomycetes) from the Patagonian Andes Forests of Argentina. Biblioteca Mycologia 201. J. Cramer.

Rajchenberg M, Gorjón SP, Pildain MB. 2011 - The phylogenetic disposition of Antrodia s.l. taxa (Polyporales, Basidiomycota) from Patagonia, Argentina. Australian Systematic Botany 24: 111-120.

Rajchenberg M, Pildain MB, Bianchinotti MV, Barroetaveña C. 2015 - The phylogenetic position of poroid Hymenochaetaceae (Hymenochaetales, Basidiomycota) from Patagonia, Argentina. Mycologia 107(4): 754-767.

Rattan S. 1977 - The resupinate Aphyllophorales of the North Western Himalayas. Biblioth. Mycol. 60:1-335.

Reid DA. 1963 - New or interesting records of Australasian Basidiomycetes: V. Kew Bulletin 17(2): 267-308.

Reid DA. 1965 - A monograph of the stipitate stereoid fungi. Beihefte zur Nova Hedwigia 18: 1388.

Reid DA. 1969 - Spring fungi in Corsica. Revue de Mycologie 33: 237-267.

Reid DA. 1979 - Tremelloscypha and Papyrodiscus, two new genera of Basidiomycetes from Australasia. Beih. Sydowia 8: 332-334.

Renvall P. 1992 - Basidiomycetes at the timberline in Lapland 4. Postia lateritia n. sp. and its rustcoloured relatives. Karstenia 32: 43-60.

Rick JE. 1934 - Monographia Thelephoracearum resupinatarum Riograndensium. Brotéria Série Trimestral: Ciências Naturais 3: 31-48.

Rick JE. 1943 - Recensentur fungi resupinati rari et novi Americae meridionalis. Lilloa 9: 215-219. 
Riebesehl J, Langer E. 2017 - Hyphodontia s.l. (Hymenochaetales, Basidiomycota) - 35 new combinations and new keys to currently all 120 species. Mycological Progress 16(6): 637666.

Roberts P. 1998 - Oliveonia and the origin of the Holobasidiomycetes. Folia Cryptogamica Estonica 33: 127-132.

Roberts P. 1999 - Rhizoctonia-forming fungi: a taxonomic guide. Royal Botanic Gardens, Kew.

Roberts P. 2000 - Corticioid fungi from Korup National Park, Cameroon. Kew Bulletin. 55(4): 803-842.

Rogers DP. 1935 - Notes on the lower Basidiomycetes. University of Iowa Studies in Natural History 17(1): 1-43.

Ryvarden L. 1972 - Radulodon, a new genus in the Corticiaceae (Basidiomycetes). Canadian Journal of Botany 50(10): 2073-2076.

Ryvarden L. 1973 - New genera in the Polyporaceae. Norwegian Journal of Botany 20(1): 1-5.

Ryvarden L. 1975 - Studies in the Aphyllophorales of Africa 2. Some new species from East Africa. Norwegian Journal of Botany 22(1): 25-34.

Ryvarden L. 1978 - Studies in the Aphyllophorales of Africa 6. Some species from eastern Central Africa. Bulletin du Jardin Botanique National de Belgique 48(1-2): 79-119.

Ryvarden L. 1991 - Genera of polypores, nomenclature and taxonomy. Synopsis Fungorum 5: 1373.

Ryvarden L. 2002 - A note on the genus Hydnodon Banker. Synopsis Fungorum 15: 31-33.

Ryvarden L. 2005 - The genus Inonotus, a synopsis. Synopsis Fungorum 21: 1-149.

Ryvarden, L. 2007 - Studies in Neotropical polypores 23. New and interesting wood-inhabiting fungi from Belize. Synopsis Fungorum. 23:32-50

Ryvarden L. 2010 - Stereoid fungi of America. Synopsis Fungorum 28: 1-206.

Ryvarden L, Gilbertson RL. 1993 - European Polypores 1, Abortiporus-Lindtneria. Fungiflora, Oslo, Norway.

Ryvarden L, Gilbertson RL. 1994 - European Polypores 2, Meripilus-Tyromyces. Fungiflora, Oslo, Norway.

Ryvarden L, Hjortstam K, Iturriaga T. 2005 - Studies in corticioid fungi from Venezuela II (Basidiomycotina, Aphyllophorales). Synopsis Fungorum 20: 42-78.

Ryvarden L, Johansen I. 1980 - A preliminary Polypore Flore of East Africa. Fungiflora, Oslo, Norway.

Ryvarden L, Melo I. 2014 - Poroid fungi of Europe. Synopsis Fungorum 31: 1-455.

Ryvarden L, Melo I. 2017 - Poroid fungi of Europe ( $2^{\text {nd }}$ ed). Synopsis Fungorum 37: 1-431.

Ryvarden L, Tutka S. 2014 - Perplexostereum Ryvarden \& Tutka nov. gen. Synopsis Fungorum 32: 72-75.

Ryvarden L, Wright JE, Rajchenberg M. 1982 - Megasporoporia, a new genus of resupinate polypores. Mycotaxon 16(1): 172-182.

Sanyal SK, Samita, Dhingra GS, Singh Avneet P. 2013 - Cordochaete (Agaricomycetes), a new corticioid genus from India. Mycotaxon 123: 103-106.

Schulzer S, Kanitz A, Knapp JA. 1866 - Die bisher bekannten Pflanzen Slavoniens, ein Versuch. Verhandlungen der Zoologisch-Botanischen Gesellschaft Wien 16: 3-172.

Sell I, Kotiranta H, Miettinen O, Põldmaa K. 2014 - Molecular analysis confirm that Botryodontia millavensis and Oxyporus philadelphi are conspecific. Mycological Progress 13: 65-74.

Sjökvist E, Larsson E, Eberhardt U et al. 2012 - Stipitate stereoid basidiocarps have evolved multiple times. Mycologia 104(5): 1046-1055.

Smith WG. 1884 - Diseases of field and garden crops. London. MacMillan.

Song J, Liu XY, Wang M, Cui BK. 2016 - Phylogeny and taxonomy of the genus Anomoloma (Amylocorticiales, Basidiomycota). Mycol Progress 15(11): 1-8.

Spirin V, Runnel K, Vlasák J, et al. 2015 - Species diversity in the Antrodia crassa group (Polyporales, Basidiomycota) inferred from morphological, geographic and DNA data. Fungal Biol. 119: 1291-1310. 
Spirin V, Vlasák J, Rivoire B et al. 2016 - Hidden species diversity in the Antrodia malicola group (Polyporales, Basidiomycota). Mycological Progress. 15(5/51): 1-12.

Spirin V, Zmitrovich IV. 2004 - A contribution to the taxonomy of corticioid fungi: Merulius Fr., Phlebia Fr., and related genera. Novosti Sistematiki Nizshikh Rastenii 37: 166-188.

Spirin V, Zmitrovich IV. 2007 - Frantisekia - a new polypore genus (Polyporales, Basidiomycota). Czech Mycology 59(2): 141-152.

Stalpers JA. 1974 - Spiniger, a new genus for imperfect states of Basidiomycetes. Proceedings van de Koninklijke Nederlandse Akademie van Wetenschappen Section C. 77(4): 402-407.

Stalpers JA. 1985 - Type studies of the species of Corticium described by G.H. Cunningham. New Zealand Journal of Botany 23: 301-310.

Stalpers JA. 2000 - The genus Ptychogaster. Karstenia. 40: 167-180.

Stalpers JA, Loerakker WM. 1982 - Laetisaria and Limonomyces species (Corticiaceae) causing pink diseases in turf grasses. Canadian Journal of Botany 60(5): 529-537.

Svrček M. 1958 - Contribution to the taxonomy of the resupinate Thelephoraceous Fungi. Ceská Mykologie 12(2): 66-77.

Tedersoo L, Harend H, Buegger et al. 2014 - Stable isotope analysis, field observations and synthesis experiments suggest that Odontia is a non-mycorrhizal sister genus of Tomentella and Thelephora. Fungal Ecology 11: 80-90.

Telleria MT. 1990 - Annotated list of the Corticiaceae, sensu lato (Aphyllophorales, Basidiomycotina), for Peninsular Spain and Balearic Islands. Bibliotheca Mycologica 135:1152.

Telleria MT, Dueñas M, Martín MP. 2017 - Three new species of Hydnophlebia (Polyporales, Basidiomycota) from the Macaronesian Islands. MycoKeys 27: 39-64.

Telleria MT, Dueñas M, Melo I et al. 2010 - A reevaluation of Hypochnicium (Polyporales) based on morphological and molecular characters. Mycologia 102(6): 1426-1436.

Telleria MT, Melo I. 1995 - Aphyllophorales resupinatae non poroides, I. AcanthobasidiumCystostereum. Flora Mycologica Iberica 1: 1-223. Madrid-Sttutgart.

Tian Y, Ghobad-Nejhad M, He SH, Dai YC. 2018 - Three new species of Aleurodiscus s.l. (Russulales, Basidiomycota) from southern China. Mycokeys 37: 93-107.

Tomšovský M, Menkis A, Vasaitis R. 2010 - Phylogenetic relationships in European Ceriporiopsis species inferred from nuclear and mitochondrial ribosomal DNA sequences. Fungal Biology 114(4): 350-358.

Vampola P. 1992 - Pouzaroporia, gen. nov. - a new genus of the polypores. Ceská Mykologie 46(1-2): 57-61.

Viner I, Spirin V, Zíbarová L, Larsson KH. 2018 - Additions to the taxonomy of Lagarobasidium and Xylodon (Hymenochaetales, Basidiomycota). MycoKeys 41: 65-90.

Wagner T, Fischer M. 2002 - Classification and phylogenetic relationships of Hymenochaete and allied genera of the Hymenochaetales, inferred from rDNA sequence data and nuclear behaviour of vegetative mycelium. Mycological Progress 1: 93-194.

Wakefield EM. 1917 - Notes on British Thelephoraceae. Transactions of the British Mycological Society 5: 474-481.

Walker J. 1996 - An opinion on the validity of the generic name Postia Fries 1874 (Eumycota: Aphyllophorales). Australasian Mycological Society Newsletter 15(2): 23-26.

Warcup JH, Talbot PHB. 1962 - Ecology and identity of mycelia isolated from soil. Transactions of the British Mycological Society 45(4): 495-518.

Westphalen MC, Rajchenberg M, Tomšovský M, Gugliotta AM. 2016 - Extensive characterization of the new genus Rickiopora (Polyporales). Fungal Biology 120(8): 1002-1009.

Westphalen MC, Rajchenberg M, Tomšovský M, Gugliotta AM. 2018 - A re-evaluation of Neotropical Junghuhnia s.l. (Polyporales, Basidiomycota) based on morphological and multigene analyses. Persoonia. 41: 130-141. 
Whitney HS, Bandoni RJ, Oberwinkler F. 1987 - Entomocorticium dendroctoni gen. et sp. nov. (Basidiomycotina), a possible nutrional symbiote of the mountain pine beetle in lodgepole pine in British Columbia. Canadian Journal of Botany 65(1): 95-102.

Wu F, Yuan Y, Chen JJ, He SH. 2016 - Luteoporia albomarginata gen. et sp. nov. (Meruliaceae, Basidiomycota) from tropical China. Phytotaxa 263(1): 31-41.

Wu SH. 1995 - Two new genera of corticioid basidiomycetes with gloeocystidia and amyloid basidiospores. Mycologia 87(6): 886-890.

Wu SH. 1996 - Studies on Gloeocystidiellum sensu lato (Basidiomycotina) in Taiwan. Mycotaxon 58: 1-68.

Wu SH, Boidin J, Chien CY. 2000 - Acanthofungus rimosus gen. et sp. nov., with reevaluation of the related genera. Mycotaxon 76: 153-161.

Wu SH, Hibbett DS, Binder M. 2001 - Phylogenetic analyses of Aleurodiscus s.l. and allied genera. Mycologia 93: 720-731.

Wu SH, Nilsson HR, Chen CT et al. 2010 - The white-rotting genus Phanerochaete is polyphyletic and distributed throughout the phleboid clade of the Polyporales (Basidiomycota). Fungal Diversity 42: 107-118.

Wu SH, Wang DM, Tschen E. 2007 - Brunneocorticium pyriforme, a new corticioid fungal genus and species belonging to the euagarics clade. Mycologia 99(2): 302-309.

Wu SH, Wang DM, Chen YP. 2017 - Purpureocorticium microsporum (Basidiomycota) gen. et sp. nov. from East Asia. Mycological Progress 17(3): 357-364.

Wu F, Yuan Y, Chen JJ et al. 2016 - Luteoporia albomarginata gen. et sp. nov. (Meruliaceae, Basidiomycota) from tropical China. Phytotaxa 263 (1): 31-41.

Wu ZQ, Shen S, Luo KY et al. 2017 - Morphological and molecular identification of a new species of Atraporiella (Polyporales, Basidiomycota) in China. Phytotaxa 332(1): 31-40.

Wu ZQ, Xu TM, Shen S et al. 2018 - Elaphroporia ailaoshanensis gen. et sp. nov. in Polyporales (Basidiomycota). Mycokeys 29: 81-95.

Yang J, Dai LD, He SH. 2016 - Hymenochaetopsis nom. nov. proposed to replace Pseudochaete (Hymenochaetales, Basidiomycota) with descriptions of $H$. laricicola sp. nov and $H$. gigasetosa new to China. Mycological Progress 15(2/13): 1-8.

Yoon SI, Kim SE, Lim YW, Jung HS. 2003 - Phylogenetic evaluation of stereoid fungi. Journal of Microbiology and Biotechnology 13: 406-414.

Yuan Y, Wu F, Dai YC et al. 2018 - Odontia aculeata and O. sparsa, two new species of tomentelloid fungi (Thelephorales, Basidiomycota) from the secondary forest of northeast China. Phytotaxa 372(3): 183-192.

Yurchenko EO. 2010 - The genus Peniophora (Basidiomycota) of Eastern Europe. Ed. Belorusskaya nauka. Minsk.

Yurchenko EO, Riebesehl J, Langer E. 2017 - Clarification of Lyomyces sambuci complex with the descriptions of four new species. Mycological Progress 16(9): 865-876.

Yurchenko EO, Riebesehl J, Langer E. 2020 - Fasciodontia gen. nov. (Hymenochaetales, Basidiomycota) and the taxonomic status of Deviodontia. Mycological Progress 19: 171-184.

Yurchenko E, Wu SH. 2016. A key to the species of Hyphodontia sensu lato. MycoKeys 12: 1-27.

Zhao CL, Cui BK, Dai YC. 2013a - New species and phylogeny of Perenniporia based on morphological and molecular characters. Fungal Diversity 58: 47-60.

Zhao CL, Cui BK, Steffen KT. 2013b - Yuchengia, a new polypore genus segregated from Perenniporia (Polyporales, Basidiomycota). Mycoscience 31: 331-338.

Zhao CL, Cui BK, Song J, Dai YC. 2015 - Fragiliporiaceae, a new family of Polyporales (Basidiomycota). Fungal Diversity 70: 115-126.

Zhao CL, Chen H, He SH, Dai, YC. 2016 - Radulotubus resupinatus gen. et sp. nov. with a poroid hymenophore in Pterulaceae (Agaricales, Basidiomycota). Nova Hedwigia. 103(1-2): 265278.

Zhao CL, Wu F, Dai YC. 2016b - Leifiporia rhizomorpha gen. et sp. nov. and L. eucalypti comb. nov. in Polyporaceae (Basidiomycota). Mycological Progress 15(7): 799-809. 
Zhao YN, Liu, SL, Nakasone KK, He SH. 2018 - Coniophoropsis bambusicola sp. nov. (Coniophoraceae, Basidiomycota) from southern Vietnam. Phytotaxa 360(2): 153-160.

Zhou LW, Dai YC. 2013 - Taxonomy and phylogeny of wood-inhabiting hydnoid species in Russulales: two new genera, three new species and two new combinations. Mycologia 105(3): 636-649.

Zmitrovich IV. 2001 - Contribution to the taxonomy of corticoid fungi. I. The genera Athelia, Byssomerulius, Hyphoderma, Odonticium. Mikologiya i Fitopatologiya 35: 9-19.

Zmitrovich IV. 2018 - Conspectus systematis Polyporacearum v. 1.0. Folia Cryptogamica Petropolitana 6: 3-145.

Zmitrovich IV, Ezhov ON, Khimich YuR. 2015 - Niemelaea, a new genus of Meruliaceae (Basidiomycota). Agriculture and Forestry Podgorica 61: 23-31.

Zmitrovich IV, Kovalenko AE. 2016 - Lentinoid and polyporoid fungi, two generic conglomerates containing important medicinal mushrooms in molecular perspective. International Journal of Medicinal Mushrooms 18(1): 23-38.

Zmitrovich IV, Malysheva VF, Spirin WA. 2006 - A new morphological arrangement of the Polyporales. I. Phanerochaetineae. Mycena 6: 4-56.

Zmitrovich IV, Spirin VA. 2002 - A contribution to the taxonomy of corticioid fungi. II. The genera Serpula, Serpulomyces gen. nov., Amylocorticiellum gen. nov. Mikologiya i Fitopatologiya. 36(1): 11-26. 